\title{
Rank 2 Bundles with Meromorphic Connections with Poles of Poincaré Rank 1
}

\author{
Claus HERTLING
}

Lehrstuhl für algebraische Geometrie, Universität Mannheim, B6, 26, 68159 Mannheim, Germany

E-mail: hertling@math.uni-mannheim.de

URL: https://www.wim.uni-mannheim.de/hertling/team/prof-dr-claus-hertling/

Received September 30, 2020, in final form August 20, 2021; Published online September 07, 2021 https://doi.org/10.3842/SIGMA.2021.082

\begin{abstract}
Holomorphic vector bundles on $\mathbb{C} \times M, M$ a complex manifold, with meromorphic connections with poles of Poincaré rank 1 along $\{0\} \times M$ arise naturally in algebraic geometry. They are called $(T E)$-structures here. This paper takes an abstract point of view. It gives a complete classification of all $(T E)$-structures of rank 2 over germs $\left(M, t^{0}\right)$ of manifolds. In the case of $M$ a point, they separate into four types. Those of three types have universal unfoldings, those of the fourth type (the logarithmic type) not. The classification of unfoldings of $(T E)$-structures of the fourth type is rich and interesting. The paper finds and lists also all $(T E)$-structures which are basic in the following sense: Together they induce all rank $2(T E)$-structures, and each of them is not induced by any other $(T E)$-structure in the list. Their base spaces $M$ turn out to be 2-dimensional $F$-manifolds with Euler fields. The paper gives also for each such $F$-manifold a classification of all rank $2(T E)$-structures over it. Also this classification is surprisingly rich. The backbone of the paper are normal forms. Though also the monodromy and the geometry of the induced Higgs fields and of the bases spaces are important and are considered.
\end{abstract}

Key words: meromorphic connections; isomonodromic deformations; (TE)-structures

2020 Mathematics Subject Classification: 34M56; 34M35; 53C07

\section{Contents}

1 Introduction $\quad 2$

2 The two-dimensional $F$-manifolds and their Euler fields $\quad 5$

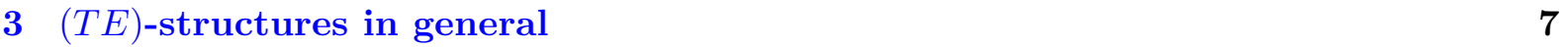

3.1 Definitions . . . . . . . . . . . . . . . . . . 7

$3.2(T E)$-structures with trace free pole part . . . . . . . . . . . . . 10

3.3 (TE)-structures over $F$-manifolds with Euler fields . . . . . . . . . . . . . 12

3.4 Birkhoff normal form . . . . . . . . . . . . . . . . . . . . 14

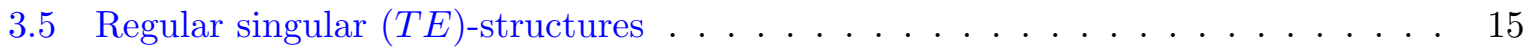

3.6 Marked $(T E)$-structures and moduli spaces for them . . . . . . . . . . . . 17

4 Rank 2 $(T E)$-structures over a point $\quad \mathbf{2 0}$

4.1 Separation into 4 cases . . . . . . . . . . . . . . . . . . 20

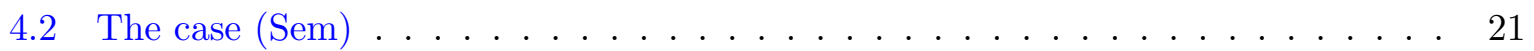

4.3 Joint considerations on the cases (Bra), (Reg) and $(\log ) \ldots \ldots . \ldots 23$

This paper is a contribution to the Special Issue on Primitive Forms and Related Topics in honor of Kyoji Saito for his 77th birthday. The full collection is available at https://www.emis.de/journals/SIGMA/Saito.html 


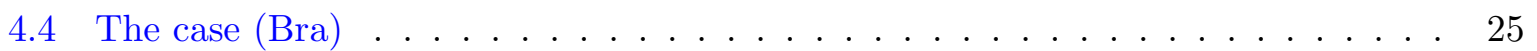

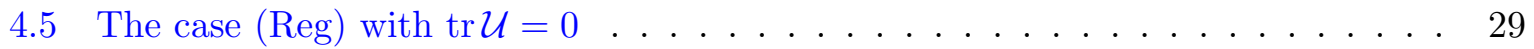

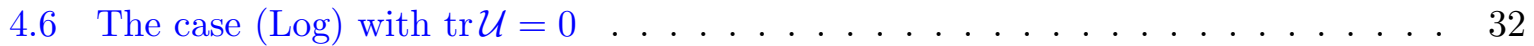

5 Rank $2(T E)$-structures over germs of regular $F$-manifolds 33

6 1-parameter unfoldings of logarithmic $(T E)$-structuresover a point $\quad 36$

6.1 Numerical invariants for such $(T E)$-structures $\ldots \ldots \ldots$. . . . . . . . . 36

6.2 1-parameter unfoldings with trace free pole part of logarithmic pure (TLE)structures over a point . . . . . . . . . . . . . . . . 41

6.3 Generically regular singular $(T E)$-structures over $(\mathbb{C}, 0)$ with logarithmic restriction over $t^{0}=0$ and not semisimple monodromy $\ldots \ldots \ldots . \ldots 47$

7 Marked regular singular rank $2(T E)$-structures $\quad 51$

8 Unfoldings of rank $2(T E)$-structures of type $(\mathrm{Log})$ over a point $\quad \mathbf{5 8}$

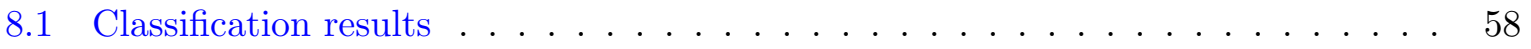

8.2 (TE)-structures over given $F$-manifolds with Euler fields . . . . . . . . . . . . 63

8.3 Proof of Theorem $8.5 \ldots \ldots \ldots 6$

9 A family of rank $3(T E)$-structures with a functional parameter $\quad \mathbf{7 0}$

$\begin{array}{ll}\text { References } & 72\end{array}$

\section{Introduction}

A holomorphic vector bundle $H$ on $\mathbb{C} \times M, M$ a complex manifold, with a meromorphic connection $\nabla$ with a pole of Poincaré rank 1 along $\{0\} \times M$ and no pole elsewhere, is called a $(T E)$-structure. The aim of this paper is the local classification of all rank $2(T E)$-structures, over arbitrary germs $\left(M, t^{0}\right)$ of manifolds.

Before we talk about the results, we will put these structures into a context, motivate their definition, mention their occurence in algebraic geometry, and formulate interesting problems. The rank 2 case is the first interesting case and already very rich. In many aspects it is probably typical for arbitrary rank, in some not. And it is certainly the only case where such a thorough classification is feasible.

The pole of Poincaré rank 1 along $\{0\} \times M$ of the pair $(H, \nabla)$ means the following. Let $t=$ $\left(t_{1}, \ldots, t_{n}\right)$ be holomorphic coordinates on $M$ with coordinate vector fields $\partial_{1}, \ldots, \partial_{n}$, and let $z$ be the standard coordinate on $\mathbb{C}$. Then $\nabla_{\partial_{z}} \sigma$ for a holomorphic section $\sigma \in \mathcal{O}(H)$ of $H$ is in $z^{-2} \mathcal{O}(H)$, and $\nabla_{\partial_{j}} \sigma$ is in $z^{-1} \mathcal{O}(H)$. The pole of order two along $\partial_{z}$ is the first case beyond the easy and tame case of a pole of order 1, i.e., a logarithmic pole. The pole of order 1 along $\partial_{i}$ gives a good variation property, a generalization of Griffiths transversality for variations of Hodge structures. It is the most natural constraint for an isomonodromic family of bundles on $\mathbb{C}$ with poles of order 2 at 0 . So, a pole of Poincaré rank 1 is in some sense the first case beyond the case of connections with logarithmic poles. (A pole of Poincaré rank $r \in \mathbb{N}_{0}$ is defined for example in [21, Section 0.14].)

In algebraic geometry, such connections arise naturally. A distinguished case is the FourierLaplace transformation (with respect to the coordinate $z$ ) of the Gauss-Manin connection of a family of holomorphic functions with isolated singularities (see [10, Chapter 8] and [21, Chapter VII]). The paper [10] defines (TERP)-structures, which are (TE)-structures with additional real structure and pairing and which generalize variations of Hodge structures. Also the notion $(T E Z P)$-structure makes sense, which is a $(T E)$-structures with a flat $\mathbb{Z}$-lattice bundle on 
$\mathbb{C}^{*} \times M$ and a certain pairing. A family of holomorphic functions with isolated singularities (and some topological well-behavedness) gives rise to a (TEZP)-structure over the base space of the family (see [9, Chapter 11.4] and [10, Chapter 8]).

In [9] and other papers of the author, a Torelli problem is considered. We formulate it here as the following question: Does the $(T E Z P)$-structure of a holomorphic function germ with an isolated singularity determine the (TEZP)-structure of the universal unfolding of the function germ? The first one is a $(T E)$-structure over a point $t^{0}$. The second one is a $(T E)$ structure over a germ $\left(M, t^{0}\right)$ of a manifold $M$. It it an unfolding of the first (TE)-structure with a primitive Higgs field. The base space $M$ is an $F$-manifold with Euler field.

We explain these notions. A second (TE)-structure over a manifold $M$ is an unfolding of a first $(T E)$-structure over a submanifold of $M$ if the restriction of the second $(T E)$-structure to the submanifold is isomorphic to the first (TE)-structure. If $\varphi: M^{\prime} \rightarrow M$ is a morphism and if $(H, \nabla)$ is a $(T E)$-structure over $M$, then the pull back $\varphi^{*}(H, \nabla)$ is a $(T E)$-structure over $M^{\prime}$. An unfolding of a $(T E)$-structure is universal if it induces any unfolding via a unique map $\varphi$ (see Definition $3.15(b)+(c)$ for details).

If $(H \rightarrow \mathbb{C} \times M, \nabla)$ is a $(T E)$-structure, then define the vector bundle $K:=\left.H\right|_{\{0\} \times M}$ on $M$ and the Higgs field $C:=[z \nabla] \in \Omega^{1}(M, \operatorname{End}(K))$ on $K$. The endomorpisms $C_{X}=$ $\left[z \nabla_{X}\right]: \mathcal{O}(K) \rightarrow \mathcal{O}(K)$ for $X \in \mathcal{T}_{M}$ commute with one another, and they commute with the endomorphism $\mathcal{U}:=\left[z^{2} \nabla_{\partial_{z}}\right]: \mathcal{O}(K) \rightarrow \mathcal{O}(K)$ (see Definition 3.8 and Lemma 3.12). The Higgs field $C$ is primitive if on each sufficiently small subset $U \subset K$ a section $\zeta_{U}$ exists such that the map $\mathcal{T}_{U} \rightarrow \mathcal{O}(K), X \mapsto C_{X} \zeta_{U}$, is an isomorphism (see Definition 3.13).

An F-manifold with Euler field is a complex manifold $M$ together with a holomorphic commutative and associative multiplication $\circ$ on $\mathcal{T}_{M}$ which comes equipped with the integrability condition (2.1), with a unit field $e \in \mathcal{T}_{M}$ (with oe $=\mathrm{id}$ ) and an Euler field $E \in \mathcal{T}_{M}$ with $\operatorname{Lie}_{E}(\circ)=\circ($ see [12] or Definition 2.1). A (TE)-structure over $M$ with primitive Higgs field induces on the base manifold $M$ the structure of an $F$-manifold with Euler field (see Theorem 3.14 for details).

A result of Malgrange [18] (cited in Theorem 3.16(c)) says that a (TE)-structure over a point $t^{0}$ has a universal unfolding if the endomorphism $\mathcal{U}: K \rightarrow K$ (here $K$ is a vector space) is regular, i.e., it has only one Jordan block for each eigenvalue. Theorem 3.16(b) gives a generalization from [13]. A special case of this generalization says that a (TE)-structure with primitive Higgs field over a germ $\left(M, t^{0}\right)$ is its own universal unfolding (see Theorem 3.16(a)). A supplement from [4] says that then the base space is a regular F-manifold (see Definition 2.4 and Theorem 2.5).

Malgrange's result gives a universal unfolding if one starts with a $(T E)$-structure over a point whose endomorphism $\mathcal{U}$ is regular. However, if one starts with a $(T E)$-structure over a point such that $\mathcal{U}$ is not regular, then in general it has no universal unfolding, and the study of all its unfoldings becomes very interesting. The second half of this paper (Sections 6-8) studies this situation in rank 2. The Torelli problem for a holomorphic function germ with an isolated singularity is similar: The endomorphism $\mathcal{U}$ of its $(T E Z P)$-structure is never regular (except if the function has an $A_{1}$-singularity), but I hope that the (TEZP)-structure determines nevertheless somehow the specific unfolding with primitive Higgs field, which comes from the universal unfolding of the original function germ.

Now sufficient background is given. We describe the contents of this paper.

The short Section 2 recalls the classification of the 2-dimensional germs of $F$-manifolds with Euler fields (Theorem 2.2 from [9] and Theorem 2.3 from [6]). It treats also regular $F$-manifolds (Definition 2.4 and Theorem 2.5 from [4]).

Section 3 recalls many general facts on $(T E)$-structures: their definition, their presentation by matrices, formal (TE)-structures, unfoldings and universal unfoldings of (TE)-structures, Malgrange's result and the generalization in [13], (TE)-structures over $F$-manifolds, $(T E)$-structures 
with primitive Higgs fields, regular singular (TE)-structures and elementary sections, Birkhoff normal form for $(T E)$-structures (not all have one, Theorem 3.20 cites existence results of Plemely and of Bolibroukh and Kostov). Not written before, but elementary is a correspondence between $(T E)$-structures with trace free endomorphism $\mathcal{U}$ and arbitrary $(T E)$-structures (Lemmata $3.9,3.10$ and 3.11).

New is the notion of a marked $(T E)$-structure. It is needed for the construction of moduli spaces. Theorem 3.29 (which builds on results in [15]) constructs such moduli spaces, but only in the case of regular singular $(T E)$-structures. It starts with a good family of regular singular $(T E)$-structures. There are two open problems. It is not clear how to generalize this notion of a good family beyond the case of regular singular $(T E)$-structures. We hope, but did not prove for rank $\geq 3$, that any regular singular $(T E$ )-structure (over $M$ with $\operatorname{dim} M \geq 1$ ) is a good family of regular singular $(T E)$-structures. For rank 2 this is true, it follows from Theorem 8.5.

Section 4 gives the classification of rank $2(T E)$-structures over a point $t^{0}$. There are 4 types, which we call (Sem), (Bra), (Reg) and (Log) (for semisimple, branched, regular singular and logarithmic). In the type (Sem) $\mathcal{U}$ has two different eigenvalues, in the type (Log) $\mathcal{U} \in \mathbb{C} \cdot \mathrm{id}$, in the types (Bra) and (Reg) $\mathcal{U}$ has a $2 \times 2$ Jordan block. In the cases when $\mathcal{U}$ is trace free, a $(T E)$-structure of type $(\mathrm{Log})$ has a logarithmic pole, a (TE)-structure of type (Reg) has a regular singular, but not logarithmic pole, and the pull back of a (TE)-structure of type (Bra) by a branched cover of $\mathbb{C}$ of order 4 has a meromorphic connection with semisimple pole of order 3 (see Lemma 4.9). The semisimple case (Sem) is not central in this paper. Therefore we do not discuss it in detail and do not introduce Stokes structures. For the other types (Bra), (Reg) and (Log), Section 4 discusses normal forms and their parameters. All (TE)-structures of type (Bra) have nice Birkhoff normal forms (Theorem 4.11), but not all of type (Reg) (Theorem 4.17 and Remark 4.19) and type (Log) (Theorem 4.20 and Remark 4.22). The types (Reg) and (Log) become transparent by the use of elementary sections.

A $(T E)$-structure of type (Sem) or (Bra) or (Reg) over a point $t^{0}$ satisfies the hypothesis of Malgrange's result, namely, the endomorphism $\mathcal{U}: K \rightarrow K$ is regular. Therefore it has a universal unfolding, and any unfolding of it is induced by this universal unfolding. Section 5 discusses this. Also because of this fact, the semisimple case is not central in this paper.

Sections 6-8 are devoted to the study of $(T E)$-structures over a germ $\left(M, t^{0}\right)$ such that the restriction to $t^{0}$ is a $(T E)$-structure of type $(\log )$. Then the set of points over which the $(T E)$-structure restricts to one of type $(\log )$ is either a hypersurface or the whole of $M$. In the first case, it restricts to a fixed generic type (Sem) or (Bra) or (Reg) over points not in the hypersurface. In the second case, the generic type is $(\mathrm{Log})$.

Section 6 starts this study. It considers the cases with trace free $\mathcal{U}$ and $\operatorname{dim} M=1$. It has three parts. In the first part, invariants of such 1-parameter families are studied. In a surprisingly direct way, constraints on the difference of the leading exponents (defined in Theorem 4.20) of the logarithmic (TE)-structure over $t^{0}$ are found, and the monodromy in the generic cases (Sem) and (Bra) turns out to be semisimple (Theorem 6.2). By Plemely's result (and our direct calculations), these cases come equipped with Birkhoff normal forms. Theorem 6.3 in the second part classifies all $(T E)$-structures over $\left(M, t^{0}\right)$ with trace free $\mathcal{U}, \operatorname{dim} M=1, \operatorname{logarithmic}$ restriction to $t^{0}$ and Birkhoff normal form. Theorem 6.7 in the third part classifies all generically regular singular $(T E)$-structures over $\left(M, t^{0}\right)$ with $\operatorname{dim} M=1$, logarithmic restriction to $t^{0}$, and whose monodromy has a $2 \times 2$ Jordan block. The majority of these cases has no Birkhoff normal form. Theorems 6.3 and 6.7 overlap in the cases which have Birkhoff normal forms.

Section 7 makes the moduli spaces of marked regular singular $(T E)$-structures from Theorem 3.28 explicit in the rank 2 cases. It builds on the classification results for the types (Reg) and (Log) in Section 4. The long Theorem 7.4 describes the moduli spaces and offers 5 figures in order to make this more transparent. The moduli spaces have countably many topological components, and each component consists of an infinite chain of projective spaces which are 
either the projective line $\mathbb{P}^{1}$ or the Hirzebruch surface $\mathbb{F}_{2}$ or $\widetilde{\mathbb{F}}_{2}$ (which is obtained by blowing down in $\mathbb{F}_{2}$ the unique (-2)-curve). These moduli spaces simplify in the generic case (Reg) the main proof in Section 8, the proof of Theorem 8.5.

Section 8 gives complete classification results, from different points of view. It has three parts. Theorem 8.1 lists all rank $2(T E)$-structures over a 2-dimensional germ $\left(M, t^{0}\right)$ such that the restriction to $t^{0}$ has a logarithmic pole, such that the Higgs field is generically primitive, and such that the induced structure of an $F$-manifold with Euler field extends to all of $M$. Theorem $8.1(d)$ offers explicit normal forms. Corollary 8.3 starts with any logarithmic rank $2(T E)$-structure over a point $t^{0}$ and lists the $(T E)$-structures in Theorem $8.1(d)$ which unfold it.

Theorem 8.5 is the most fundamental result of Section 8. Table (8.4) in it is a sublist of the $(T E)$-structures in Theorem 8.1 $(d)$. Theorem 8.5 states that any unfolding of a rank $2(T E)$ structure of type $(\mathrm{Log})$ over a point is induced by one (TE)-structure in table (8.4). In the generic cases $(\operatorname{Reg})$ and $(\log )$ these are precisely those in Theorem 8.1 $(d)$ with primitive Higgs field, but in the generic cases (Sem) and (Bra) table (8.4) contains many (TE)-structures with only generically primitive Higgs field. All the (TE)-structures in table (8.4) are universal unfoldings of themselves, also those with only generically primitive Higgs field. Almost all logarithmic $(T E)$-structures over a point have several unfoldings which do not induce one another. Only the logarithmic (TE)-structures over a point whose monodromy has a $2 \times 2$ Jordan block and whose two leading exponents coincide have a universal unfolding. This follows from Theorem 8.5 and Corollary 8.3.

The second part of Section 8 starts from the 2-dimensional $F$-manifolds with Euler fields and discusses how many and which (TE)-structures exist over each of them. It turns out that the nilpotent $F$-manifold $\mathcal{N}_{2}$ with the Euler field $E=t_{1} \partial_{1}+t_{2}^{r}\left(1+c_{3} t_{2}^{r-1}\right) \partial_{2}$ for $r \geq 2$ (case (2.7) in Theorem 2.3) does not have any $(T E)$-structure over it if $c_{3} \neq 0$, and it has no (TE)-structure with primitive Higgs field over it if $c_{3} \neq 0$ or $r \geq 3$. However, most 2-dimensional $F$-manifolds with Euler fields have one or countably many families of $(T E)$-structures with 1 or 2 parameters over them.

The third part of Section 8 is the proof of Theorem 8.5.

In many aspects, the $(T E)$-structures of rank 2 are probably typical also for higher rank. But Section 9 makes one phenomenon explicit which arises only in rank $\geq 3$. Section 9 presents a family of rank $3(T E)$-structures with primitive Higgs fields over a fixed 3-dimensional globally irreducible $F$-manifold with nowhere regular Euler field, such that the family has a functional parameter. The example is essentially due to M. Saito, it is a Fourier-Laplace transformation of the main example in a preliminary version of [23] (though he considers only the bundle and connection over a 2-dimensional submanifold of the $F$-manifold).

This paper has some overlap with [6] and [7]. In [6] (TE)-structures over the 2-dimensional $F$-manifold $\mathcal{N}_{2}$ (with all possible Euler fields) were studied. They are of generic types (Bra), $(\mathrm{Reg})$ or $(\mathrm{Log})$. In [7, Chapter 8] $(T E)$-structures over the 2-dimensional $F$-manifolds $I_{2}(m)$ were studied. They are of generic type (Sem). However, in [6] and [7] the focus was on (TE)structures with primitive Higgs fields. Those with generically primitive, but not primitive Higgs fields were not considered. And the approach to the classification was very different. It relied on the formal classification of rank $2(T)$-structures in [5]. The approach here is independent of these three papers.

\section{The two-dimensional $\boldsymbol{F}$-manifolds and their Euler fields}

F-manifolds were first defined in [12]. Their basic properties were developed in [9]. An overview on them and on more recent results is given in [7]. 


\section{Definition 2.1.}

(a) An $F$-manifold $(M, \circ, e)$ (without Euler field) is a complex manifold $M$ with a holomorphic commutative and associative multiplication $\circ$ on the holomorphic tangent bundle $T M$, and with a global holomorphic vector field $e \in \mathcal{T}_{M}$ with $e o=\operatorname{id~(~} e$ is called a unit field), which satisfies the following integrability condition:

$$
\operatorname{Lie}_{X \circ Y}(\circ)=X \circ \operatorname{Lie}_{Y}(\circ)+Y \circ \operatorname{Lie}_{X}(\circ) \quad \text { for } \quad X, Y \in \mathcal{T}_{M}
$$

(b) Given an $F$-manifold $(M, \circ, e)$, an Euler field on it is a global vector field $E \in \mathcal{T}_{M}$ with $\operatorname{Lie}_{E}(0)=0$.

In this paper we are mainly interested in the 2-dimensional $F$-manifolds and their Euler fields. They were classified in [9].

Theorem 2.2 ([9, Theorem 4.7]). In dimension 2, (up to isomorphism) the germs of Fmanifolds fall into three types:

(a) The semisimple germ. It is called $A_{1}^{2}$, and it can be given as follows

$$
\begin{aligned}
& (M, 0)=\left(\mathbb{C}^{2}, 0\right) \quad \text { with coordinates } \quad u=\left(u_{1}, u_{2}\right) \quad \text { and } \quad e_{k}=\frac{\partial}{\partial u_{k}} \\
& e=e_{1}+e_{2}, \quad e_{j} \circ e_{k}=\delta_{j k} \cdot e_{j} .
\end{aligned}
$$

Any Euler field takes the shape

$$
E=\left(u_{1}+c_{1}\right) e_{1}+\left(u_{2}+c_{2}\right) e_{2} \quad \text { for some } \quad c_{1}, c_{2} \in \mathbb{C} .
$$

(b) Irreducible germs, which (i.e., some holomorphic representatives of them) are at generic points semisimple. They form a series $I_{2}(m), m \in \mathbb{Z}_{\geq 3}$. The germ of type $I_{2}(m)$ can be given as follows

$$
\begin{aligned}
& (M, 0)=\left(\mathbb{C}^{2}, 0\right) \quad \text { with coordinates } t=\left(t_{1}, t_{2}\right) \quad \text { and } \partial_{k}:=\frac{\partial}{\partial t_{k}}, \\
& e=\partial_{1}, \quad \partial_{2} \circ \partial_{2}=t_{2}^{m-2} e .
\end{aligned}
$$

Any Euler field takes the shape

$$
E=\left(t_{1}+c_{1}\right) \partial_{1}+\frac{2}{m} t_{2} \partial_{2} \quad \text { for some } \quad c_{1} \in \mathbb{C} .
$$

(c) An irreducible germ, such that the multiplication is everywhere irreducible. It is called $\mathcal{N}_{2}$, and it can be given as follows

$$
\begin{aligned}
& (M, 0)=\left(\mathbb{C}^{2}, 0\right) \quad \text { with coordinates } t=\left(t_{1}, t_{2}\right) \text { and } \partial_{k}:=\frac{\partial}{\partial t_{k}}, \\
& e=\partial_{1}, \quad \partial_{2} \circ \partial_{2}=0 .
\end{aligned}
$$

Any Euler field takes the shape

$$
\begin{aligned}
& E=\left(t_{1}+c_{1}\right) \partial_{1}+g\left(t_{2}\right) \partial_{2} \quad \text { for some } \quad c_{1} \in \mathbb{C} \\
& \text { and some function } g\left(t_{2}\right) \in \mathbb{C}\left\{t_{2}\right\} .
\end{aligned}
$$


The family of Euler fields in (2.3) on $\mathcal{N}_{2}$ can be reduced by coordinate changes, which respect the multiplication of $\mathcal{N}_{2}$, to a family with two continuous parameters and one discrete parameter. This classification is proved in [6]. It is recalled in Theorem 2.3. The group Aut $\left(\mathcal{N}_{2}\right)$ of automorphisms of the germ $\mathcal{N}_{2}$ of an $F$-manifold is the group of coordinate changes of $\left(\mathbb{C}^{2}, 0\right)$ which respect the multiplication of $\mathcal{N}_{2}$. It is

$$
\operatorname{Aut}\left(\mathcal{N}_{2}\right)=\left\{\left(t_{1}, t_{2}\right) \mapsto\left(t_{1}, \lambda\left(t_{2}\right)\right) \mid \lambda \in \mathbb{C}\left\{t_{2}\right\} \text { with } \lambda^{\prime}(0) \neq 0 \text { and } \lambda(0)=0\right\} .
$$

Theorem 2.3. Any Euler field on the germ $\mathcal{N}_{2}$ of an F-manifold can be brought by a coordinate change in $\operatorname{Aut}\left(\mathcal{N}_{2}\right)$ to a unique one in the following family of Euler fields

$$
\begin{aligned}
& E=\left(t_{1}+c_{1}\right) \partial_{1}+\partial_{2}, \\
& E=\left(t_{1}+c_{1}\right) \partial_{1}, \\
& E=\left(t_{1}+c_{1}\right) \partial_{1}+c_{2} t_{2} \partial_{2}, \\
& E=\left(t_{1}+c_{1}\right) \partial_{1}+t_{2}^{r}\left(1+c_{3} t_{2}^{r-1}\right) \partial_{2},
\end{aligned}
$$

where $c_{1}, c_{3} \in \mathbb{C}, c_{2} \in \mathbb{C}^{*}$ and $r \in \mathbb{Z}_{\geq 2}$. The group $\operatorname{Aut}\left(\mathcal{N}_{2}, E\right)$ of coordinate changes of $\left(\mathbb{C}^{2}, 0\right)$ which respect the multiplication of $\mathcal{N}_{2}$ and this Euler field is

$\operatorname{Aut}\left(\mathcal{N}_{2}, E\right)=\left\{\left(t_{1}, t_{2}\right) \mapsto\left(t_{1}, \gamma\left(t_{2}\right) t_{2}\right) \mid \gamma\right.$ as in $\left.(2.8)\right\}$,

\begin{tabular}{c|c|c|c|c}
\hline Case & $(2.4)$ & $(2.5)$ & $(2.6)$ & $(2.7)$ \\
\hline$\gamma \in$ & $\{1\}$ & $\mathbb{C}\left\{t_{2}\right\}^{*}$ & $\mathbb{C}^{*}$ & $\left\{\mathrm{e}^{2 \pi \mathrm{i} l /(r-1)} \mid l \in \mathbb{Z}\right\}$ \\
\hline
\end{tabular}

A special class of $F$-manifolds, the regular $F$-manifolds, is related to a result of Malgrange on universal unfoldings of (TE)-structures, see Remarks 3.17.

Definition 2.4 ([4, Definition 1.2]). A regular $F$-manifold is an $F$-manifold $(M, \circ, e)$ with Euler field $E$ such that at each $t \in M$ the endomorphism $\left.E \circ\right|_{t}: T_{t} M \rightarrow T_{t} M$ is a regular endomorphism, i.e., it has for each eigenvalue only one Jordan block.

Theorem 2.5 ([4, Theorem 1.3(ii)]). For each regular endomorphism of a finite dimensional $\mathbb{C}$ vector space, there is a unique (up to unique isomorphism) germ $\left(M, t^{0}\right)$ of a regular $F$-manifold such that $\left.E \circ\right|_{t^{0}}$ is isomorphic to this endomorphism.

\section{Remarks 2.6.}

(i) For a normal form of this germ of an $F$-manifold, see [4, Theorem 1.3(i)].

(ii) In dimension 2, this theorem is an easy consequence of Theorems 2.2 and 2.3. The germs of regular 2-dimensional $F$-manifolds are as follows:

(a) The germ $A_{1}^{2}$ in Theorem 2.2(a) with any Euler field $E=\left(u_{1}+c_{1}\right) e_{1}+\left(u_{2}+c_{2}\right) e_{2}$ as in (2.2) with $c_{1}, c_{2} \in \mathbb{C}, c_{1} \neq c_{2}$.

(b) The germ $\mathcal{N}_{2}$ in Theorem 2.2(c) with any Euler field $E=\left(t_{1}+c_{1}\right) \partial_{1}+\partial_{2}$ as in (2.4) with $c_{1} \in \mathbb{C}$.

\section{$3 \quad(T E)$-structures in general}

\subsection{Definitions}

A $(T E)$-structure is a holomorphic vector bundle on $\mathbb{C} \times M, M$ a complex manifold, with a meromorphic connection $\nabla$ with a pole of Poincaré rank 1 along $\{0\} \times M$ and no pole elsewhere. Here we consider them together with the weaker notion of $(T)$-structure and the more rigid notions of a $(T L)$-structure and a $(T L E)$-structure. The structures had been considered before in [13], and they are related to structures in [21, Chapter VII] and in [20]. 


\section{Definition 3.1.}

(a) Definition of a $(T)$-structure $(H \rightarrow \mathbb{C} \times M, \nabla): H \rightarrow \mathbb{C} \times M$ is a holomorphic vector bundle. $\nabla$ is a map

$$
\nabla: \mathcal{O}(H) \rightarrow z^{-1} \mathcal{O}_{\mathbb{C} \times M} \cdot \Omega_{M}^{1} \otimes \mathcal{O}(H)
$$

which satisfies the Leibniz rule,

$$
\nabla_{X}(a \cdot s)=X(a) \cdot s+a \cdot \nabla_{X} s \quad \text { for } \quad X \in \mathcal{T}_{M}, \quad a \in \mathcal{O}_{\mathbb{C} \times M}, \quad s \in \mathcal{O}(H),
$$

and which is flat (with respect to $X \in \mathcal{T}_{M}$, not with respect to $\partial_{z}$ ),

$$
\nabla_{X} \nabla_{Y}-\nabla_{Y} \nabla_{X}=\nabla_{[X, Y]} \quad \text { for } \quad X, Y \in \mathcal{T}_{M}
$$

Equivalent: For any $z \in \mathbb{C}^{*}$, the restriction of $\nabla$ to $\left.H\right|_{\{z\} \times M}$ is a flat holomorphic connection.

(b) Definition of a $(T E)$-structure $(H \rightarrow \mathbb{C} \times M, \nabla): H \rightarrow \mathbb{C} \times M$ is a holomorphic vector bundle. $\nabla$ is a flat connection on $\left.H\right|_{\mathbb{C}^{*} \times M}$ with a pole of Poincaré rank 1 along $\{0\} \times M$, so it is a map

$$
\nabla: \mathcal{O}(H) \rightarrow\left(z^{-1} \mathcal{O}_{\mathbb{C} \times M} \cdot \Omega_{M}^{1}+z^{-2} \mathcal{O}_{\mathbb{C} \times M} \cdot \mathrm{d} z\right) \otimes \mathcal{O}(H)
$$

which satisfies the Leibniz rule and is flat.

(c) Definition of a $(T L)$-structure $\left(H \rightarrow \mathbb{P}^{1} \times M, \nabla\right): H \rightarrow \mathbb{P}^{1} \times M$ is a holomorphic vector bundle. $\nabla$ is a map

$$
\nabla: \mathcal{O}(H) \rightarrow\left(z^{-1} \mathcal{O}_{\mathbb{P}^{1} \times M}+\mathcal{O}_{\mathbb{P}^{1} \times M}\right) \cdot \Omega_{M}^{1} \otimes \mathcal{O}(H),
$$

such that for any $z \in \mathbb{P}^{1} \backslash\{0\}$, the restriction of $\nabla$ to $\left.H\right|_{\{z\} \times M}$ is a flat connection. It is called pure if for any $t \in M$ the restriction $\left.H\right|_{\mathbb{P}^{1} \times\{t\}}$ is a trivial holomorphic bundle on $\mathbb{P}^{1}$.

(d) Definition of a $(T L E)$-structure $\left(H \rightarrow \mathbb{P}^{1} \times M, \nabla\right)$ : It is simultaneously a $(T E)$-structure and a $(T L)$-structure, where the connection $\nabla$ has a logarithmic pole along $\{\infty\} \times M$. The (TLE)-structure is called pure if the $(T L)$-structure is pure.

Remark 3.2. Here we write the data in Definition 3.1(a)-(b) and the compatibility conditions between them in terms of matrices. Consider a $(T E)$-structure $(H \rightarrow \mathbb{C} \times M, \nabla)$ of rank $\operatorname{rk} H=r \in \mathbb{N}$. We will fix the notations for a trivialization of the bundle $\left.H\right|_{U \times M}$ for some small neighborhood $U \subset \mathbb{C}$ of 0 . Trivialization means the choice of a basis $\underline{v}=\left(v_{1}, \ldots, v_{r}\right)$ of the bundle $\left.H\right|_{U \times M}$. Also, we choose local coordinates $t=\left(t_{1}, \ldots, t_{n}\right)$ with coordinate vector fields $\partial_{i}=\partial / \partial t_{i}$ on $M$. We write

$$
\begin{aligned}
& \nabla \underline{v}=\underline{v} \cdot \Omega \quad \text { with } \quad \Omega=\sum_{i=1}^{r} z^{-1} \cdot A_{i}(z, t) \mathrm{d} t_{i}+z^{-2} B(z, t) \mathrm{d} z \\
& A_{i}(z, t)=\sum_{k \geq 0} A_{i}^{(k)} z^{k} \in M_{r \times r}\left(\mathcal{O}_{U \times M}\right) \\
& B(z, t)=\sum_{k \geq 0} B^{(k)} z^{k} \in M_{r \times r}\left(\mathcal{O}_{U \times M}\right),
\end{aligned}
$$

with $A_{i}^{(k)}, B^{(k)} \in M_{r \times r}\left(\mathcal{O}_{M}\right)$, but this dependence on $t \in M$ is usually not written explicity. The flatness $0=\mathrm{d} \Omega+\Omega \wedge \Omega$ of the connection $\nabla$ says for $i, j \in\{1, \ldots, n\}$ with $i \neq j$

$$
\begin{aligned}
& 0=z \partial_{i} A_{j}-z \partial_{j} A_{i}+\left[A_{i}, A_{j}\right], \\
& 0=z \partial_{i} B-z^{2} \partial_{z} A_{i}+z A_{i}+\left[A_{i}, B\right] .
\end{aligned}
$$


These equations split into the parts for the different powers $z^{k}$ for $k \geq 0$ as follows (with $\left.A_{i}^{(-1)}=B^{(-1)}=0\right)$,

$$
\begin{aligned}
& 0=\partial_{i} A_{j}^{(k-1)}-\partial_{j} A_{i}^{(k-1)}+\sum_{l=0}^{k}\left[A_{i}^{(l)}, A_{j}^{(k-l)}\right], \\
& 0=\partial_{i} B^{(k-1)}-(k-2) A_{i}^{(k-1)}+\sum_{l=0}^{k}\left[A_{i}^{(l)}, B^{(k-l)}\right] .
\end{aligned}
$$

In the case of a $(T)$-structure, $B$ and all equations except (3.2) which contain $B$ are dropped.

Consider a second $(T E)$-structure $(\widetilde{H} \rightarrow \mathbb{C} \times M, \widetilde{\nabla})$ of rank $r$ over $M$, where all data except $M$ are written with a tilde. Let $\underline{v}$ and $\underline{\widetilde{v}}$ be trivializations. A holomorphic isomorphism from the first to the second $(T E)$-structure maps $\underline{v} \cdot T$ to $\underline{\underline{v}}$, where $T=T(z, t)=\sum_{k \geq 0} T^{(k)} z^{k} \in$ $M_{r \times r}\left(\mathcal{O}_{(\mathbb{C}, 0) \times M}\right)$ with $T^{(k)} \in M_{r \times r}\left(\mathcal{O}_{M}\right)$ and $T^{(0)}$ invertible satisfies

$$
\underline{v} \cdot \Omega \cdot T+\underline{v} \cdot \mathrm{d} T=\nabla(\underline{v} \cdot T)=\underline{v} \cdot T \cdot \widetilde{\Omega} .
$$

Equation (3.9) says more explicitly

$$
\begin{aligned}
& 0=z \partial_{i} T+A_{i} \cdot T-T \cdot \widetilde{A}_{i}, \\
& 0=z^{2} \partial_{z} T+B \cdot T-T \cdot \widetilde{B} .
\end{aligned}
$$

These equations split into the parts for the different powers $z^{k}$ for $k \geq 0$ as follows (with $\left.T^{(-1)}:=0\right)$ :

$$
\begin{aligned}
& 0=\partial_{i} T^{(k-1)}+\sum_{l=0}^{k}\left(A_{i}^{(l)} \cdot T^{(k-l)}-T^{(k-l)} \cdot \widetilde{A}_{i}^{(l)}\right), \\
& 0=(k-1) T^{(k-1)}+\sum_{l=0}^{k}\left(B^{(l)} \cdot T^{(k-l)}-T^{(k-l)} \cdot \widetilde{B}^{(l)}\right) .
\end{aligned}
$$

The isomorphism here fixes the base manifold $M$. Such isomorphisms are called gauge isomorphisms. A general isomorphism is a composition of a gauge isomorphism and a coordinate change on $M$ (a coordinate change induces an isomorphism of (TE)-structures, see Lemma 3.6).

Remark 3.3. In this paper we care mainly about $(T E)$-structures over the 2-dimensional germs of $F$-manifolds with Euler fields. For each of them except $\left(\mathcal{N}_{2}, E=\left(t_{1}+c_{1}\right) \partial_{1}\right)$, the group of coordinate changes of $(M, 0)=\left(\mathbb{C}^{2}, 0\right)$ which respect the multiplication and $E$ is quite small, see Theorem 2.3. Therefore in this paper, we care mainly about gauge isomorphisms of the (TE)-structures over these $F$-manifolds with Euler fields.

Definition 3.4. Let $M$ be a complex manifold.

(a) The sheaf $\mathcal{O}_{M}[[z]]$ on $M$ is defined by $\mathcal{O}_{M}[[z]](U):=\mathcal{O}_{M}(U)[[z]]$ for an open subset $U \subset M$ (with $\mathcal{O}_{M}(U)$ and $\mathcal{O}_{M}[[z]](U)$ the sections of $\mathcal{O}_{M}$ and $\mathcal{O}_{M}[[z]]$ on $U$ ). Observe that the germ $\left(\mathcal{O}_{M}[[z]]\right)_{t^{0}}$ for $t^{0} \in M$ consists of formal power series $\sum_{k \geq 0} f_{k} z^{k}$ whose coefficients $f_{k} \in \mathcal{O}_{M, t^{0}}$ have a common convergence domain. In the case of $\left(M, t^{0}\right)=\left(\mathbb{C}^{n}, 0\right)$ we write $\left.\mathcal{O}_{\mathbb{C}^{n}}[[z]]_{0}=: \mathbb{C}\{t, z]\right]$.

(b) A formal $(T)$-structure over $M$ is a free $\mathcal{O}_{M}[[z]]$-module $\mathcal{O}(H)$ of some finite rank $r \in \mathbb{N}$ together with a map $\nabla$ as in (3.1), where $\mathcal{O}_{\mathbb{C} \times M}$ is replaced by $\mathcal{O}_{M}[[z]]$ which satisfies properties analogous to $\nabla$ in Definition 3.1(a), i.e., the Leibniz rule for $X \in \mathcal{T}_{M}, a \in \mathcal{O}_{M}[[z]]$, $s \in \mathcal{O}(H)$ and the flatness condition for $X, Y \in \mathcal{T}_{M}$.

A formal (TE)-structure is defined analogously: In Definition 3.1(b) one has to replace $\mathcal{O}_{\mathbb{C} \times M}$ by $\mathcal{O}_{M}[[z]]$. 
Remark 3.5. The formulas in Remark 3.2 hold also for formal $(T)$-structures and formal $(T E)$ structures if one replaces $\mathcal{O}_{\mathbb{C} \times M}, \mathcal{O}_{U \times M}$ and $\mathcal{O}_{(\mathbb{C}, 0) \times M}$ by $\mathcal{O}_{M}[[z]]$.

The following lemma is obvious.

Lemma 3.6. Let $(H \rightarrow \mathbb{C} \times M, \nabla)$ be a (TE)-structure over $M$, and let $\varphi: M^{\prime} \rightarrow M$ be a holomorphic map between manifolds. One can pull back $H$ and $\nabla$ with id $\times \varphi: \mathbb{C} \times M^{\prime} \rightarrow \mathbb{C} \times M$. We call the pull back $\varphi^{*}(H, \nabla)$. It is a $(T E)$-structure over $M^{\prime}$. We say that the pull back $\varphi^{*}(H, \nabla)$ is induced by the $(T E)$-structure $(H, \nabla)$ via the map $\varphi$.

\section{Remarks 3.7.}

(i) We will give in Theorem 8.5 and in Corollary 5.1 and Lemma 5.2(iv) a classification of rank $2(T E)$-structures over germs $\left(M, t^{0}\right)=\left(\mathbb{C}^{2}, 0\right)$ of 2-dimensional manifolds such that any rank $2(T E)$-structure over a germ $\left(M^{\prime}, s^{0}\right)$ is obtained as the pull back $\varphi^{*}(H, \nabla)$ of a rank $2(T E)$-structure in the classification via a holomorphic map $\varphi:\left(M^{\prime}, s^{0}\right) \rightarrow\left(M, t^{0}\right)$.

(ii) Here the behaviour of the $(T E)$-structure $(H, \nabla)$ over $\left(M, t^{0}\right)=\left(\mathbb{C}^{2}, 0\right)$ with coordinates $t=\left(t_{1}, t_{2}\right)$ along $t_{1}$ is quite trivial. It is convenient to split it off. The next subsection does this in greater generality.

\section{$3.2(T E)$-structures with trace free pole part}

Definition 3.8. Let $(H \rightarrow \mathbb{C} \times M, \nabla)$ be a $(T E)$-structure. Define the vector bundle $K:=$ $\left.H\right|_{\{0\} \times M}$ over $M$. The pole part of the $(T E)$-structure is the endomorphism $\mathcal{U}: K \rightarrow K$ which is defined by

$$
\mathcal{U}:=\left[z^{2} \nabla_{\partial_{z}}\right]: K \rightarrow K
$$

The pole part is trace free if $\operatorname{tr} \mathcal{U}=0$ on $M$.

The following lemma gives formal invariants of a $(T E)$-structure.

Lemma 3.9. Let $(H \rightarrow \mathbb{C} \times M, \nabla)$ be a (TE)-structure of rank $r \in \mathbb{N}$ over a manifold $M$. By a formal invariant of the (TE)-structure, we mean an invariant of its formal isomorphism class.

(a) Its pole part $\mathcal{U}$, that means the pair $(K, \mathcal{U})$ up to isomorphism, is a formal invariant of the (TE)-structure. Especially, the holomorphic functions $\delta^{(0)}:=\operatorname{det} \mathcal{U} \in \mathcal{O}_{M}$ and $\rho^{(0)}:=\frac{1}{r} \operatorname{tr} \mathcal{U} \in \mathcal{O}_{M}$ are formal invariants.

(b) For any $t^{0} \in M$, fix an $\mathcal{O}_{M, t^{0}}$-basis $\underline{v}$ of $\mathcal{O}(H)_{\left(0, t^{0}\right)}$, consider the matrices in (3.2)-(3.4), consider the function $\rho^{(1)}:=\frac{1}{r} \operatorname{tr} B^{(1)} \in \mathcal{O}_{M, t^{0}}$, and consider the functions $\delta^{(k)} \in \mathcal{O}_{M, t^{0}}$ for $k \in \mathbb{N}_{0}$ which are defined by writing $\operatorname{det} B$ as a power series

$$
\operatorname{det} B=\sum_{k \geq 0} \delta^{(k)} z^{k}
$$

Then the functions $\delta^{(1)}$ and $\rho^{(1)}$ are independent of the choice of the basis $\underline{v}$. The locally for any $t^{0}$ defined functions $\delta^{(1)}$ and $\rho^{(1)}$ glue to global holomorphic functions $\delta^{(1)} \in \mathcal{O}_{M}$ and $\rho^{(1)} \in \mathcal{O}_{M}$. They are formal invariants. Furthermore, the function $\rho^{(1)}$ is constant on any component of $M$. 
Proof. $\mathcal{U}, \delta^{(0)}, \rho^{(0)}$ and $\delta^{(1)}$ are formal invariants because of (3.11): $\widetilde{B}=T^{-1} B T+z^{2} \cdot T^{-1} \partial_{z} T$. For $\rho^{(1)}$, observe additionally

$$
\widetilde{B}^{(1)}=\left(T^{(0)}\right)^{-1} B^{(1)} T^{(0)}+\left[\left(T^{(0)}\right)^{-1} B^{(0)} T^{(0)},\left(T^{(0)}\right)^{-1} T^{(1)}\right] .
$$

Recall also that the trace of a commutator of matrices is 0 . Therefore $\rho^{(1)}$ is a formal invariant. Equation (3.8) for $k=2$ implies $\partial_{i} \operatorname{tr}\left(B^{(1)}\right)=0$, so the function $\rho^{(1)}$ is constant.

The following lemma is obvious.

Lemma 3.10. Let $(H \rightarrow \mathbb{C} \times M, \nabla)$ be a $(T E)$-structure of rank $r \in \mathbb{N}$ over a manifold $M$.

(a) Consider a holomorphic function $g: M \rightarrow \mathbb{C}$. The trivial line bundle $H^{[1]}=\mathbb{C} \times(\mathbb{C} \times M) \rightarrow$ $\mathbb{C} \times M$ over $\mathbb{C} \times M$ with connection $\nabla^{[1]}:=\mathrm{d}+\mathrm{d}\left(\frac{g}{z}\right)$ defines a $(T E)$-structure of rank 1 over $M$, whose sheaf of sections with connection is called $\mathcal{E}^{g / z}$.

(b) $(\mathcal{O}(H), \nabla) \otimes \mathcal{E}^{g / z}$ for $g$ as in $(a)$ is a $(T E)$-structure.

(c) The (TE)-structure $\left(H^{[2]} \rightarrow \mathbb{C} \times M, \nabla^{[2]}\right)$ with $\left(\mathcal{O}\left(H^{[2]}\right), \nabla^{[2]}\right)=(\mathcal{O}(H), \nabla) \otimes \mathcal{E}^{\rho^{(0)} / z}$ has trace free pole part. And, of course, $(\mathcal{O}(H), \nabla) \cong\left(\mathcal{O}\left(H^{[2]}\right), \nabla^{[2]}\right) \otimes \mathcal{E}^{-\rho^{(0)} / z}$. If $\underline{v}$ is a $\mathbb{C}\{t, z\}$-basis of $\mathcal{O}(H)_{0}=\mathcal{O}\left(H^{[2]}\right)_{0}$, then the matrix valued connection 1 -forms $\Omega$ and $\Omega^{[2]}$ of $\nabla$ and $\nabla^{[2]}$ with respect to this basis satisfy $\Omega=\Omega^{[2]}-\mathrm{d}\left(\frac{\rho^{(0)}}{z}\right) \cdot \mathbf{1}_{r}$.

(d) (Definition) Consider a (TE)-structure $\left(H^{[3]} \rightarrow \mathbb{C} \times M^{[3]}, \nabla^{[3]}\right)$ with trace free pole part. Consider the manifold $M^{[4]}:=\mathbb{C} \times M^{[3]}$ with (local) coordinates $t_{1}$ on $\mathbb{C}$ and $t^{\prime}$ on $M^{[3]}$, and the projection $\varphi^{[4]}: M^{[4]} \rightarrow M^{[3]},\left(t_{1}, t^{\prime}\right) \mapsto t^{\prime}$. Define the $\left(\right.$ TE)-structure $\left(H^{[4]} \rightarrow\right.$ $\left.\mathbb{C} \times M^{[4]}, \nabla^{[4]}\right)$ with $\left(\mathcal{O}\left(H^{[4]}\right), \nabla^{[4]}\right)=\left(\varphi^{[4]}\right)^{*}\left(\mathcal{O}\left(H^{[3]}\right), \nabla^{[3]}\right) \otimes \mathcal{E}^{t_{1} / z}$.

(e) If the $(T E)$-structure $\left(H^{[2]}, \nabla^{[2]}\right)$ is induced by the $(T E)$-structure $\left(H^{[3]}, \nabla^{[3]}\right)$ via a map $\varphi: M \rightarrow M^{[3]}$, then the $(T E)$-structure $(H, \nabla)$ is induced by the $(T E)$-structure $\left(H^{[4]}, \nabla^{[4]}\right)$ via the $\operatorname{map}\left(-\rho^{(0)}, \varphi\right): M \rightarrow M^{[4]}=\mathbb{C} \times M^{[3]}$.

Part $(c)$ allows to go from an arbitrary $(T E)$-structure to one with trace free pole part, and to go back to the original one. Part $(e)$ considers two $(T E)$-structures as in part $(c)$, an original one and an associated one with trace free pole part. If the associated one is induced by a third $(T E)$-structure, then the original one is induced by a closely related ( $T E$ )-structure with one parameter more. Lemma 3.11 continues Lemma 3.10.

Lemma 3.11. Let $\left(H \rightarrow \mathbb{C} \times\left(M, t^{0}\right), \nabla\right)$ be a $(T E)$-structure of rank $r \in \mathbb{N}$ over a germ $\left(M, t^{0}\right)$ of a manifold, with coordinates $t=\left(t_{1}, \ldots, t_{n}\right)$ and $\partial_{i}:=\partial / \partial t_{i}$. We suppose $t^{0}=0$ so that $\mathcal{O}_{\left(\mathbb{C} \times M,\left(0, t^{0}\right)\right)}=\mathbb{C}\{t, z\}$. Recall the functions $\rho^{(0)}$ and $\rho^{(1)}$ of the $(T E)$-structure from Lemma 3.9.

Consider the $(T E)$-structure $\left(H^{[2]}, \nabla^{[2]}\right)$ from Lemma 3.10 with trace free pole part which is defined by $\left(\mathcal{O}\left(H^{[2]}\right), \nabla^{[2]}\right):=(\mathcal{O}(H), \nabla) \otimes \mathcal{E}^{\rho^{(0)} / z}$. Here $H^{[2]}=H$, but $\nabla^{[2]}=\nabla+\mathrm{d}\left(\frac{\rho^{(0)}}{z}\right) \cdot \mathrm{id}$. The matrices $A_{i}$ and $B$ in (3.2)-(3.4) for the (TE)-structure $\left(H^{[2]}, \nabla^{[2]}\right)$ of any $\mathbb{C}\{t, z\}$-basis $\underline{v}$ of $\mathcal{O}\left(H^{[2]}\right)_{0}$ satisfy

$$
0=\operatorname{tr} A_{i}^{(0)}=\operatorname{tr} B^{(0)}=\operatorname{tr}\left(B^{(1)}-\rho^{(1)} \mathbf{1}_{r}\right) .
$$

The basis $\underline{v}$ can be chosen such that the matrices satisfy

$$
0=\operatorname{tr} A_{i}=\operatorname{tr}\left(B-z \rho^{(1)} \mathbf{1}_{r}\right) .
$$


Proof. Any $\mathbb{C}\{t, z\}$-basis $\underline{v}$ of $\mathcal{O}\left(H^{[2]}\right)_{0}=\mathcal{O}(H)_{0}$ satisfies

$\operatorname{tr} B^{(0)}=\operatorname{tr} \mathcal{U}^{[2]}=0 \quad$ as $\left(H^{[2]}, \nabla^{[2]}\right)$ has trace free pole part,

$\operatorname{tr} A_{i}^{(0)}=0 \quad$ because of $\operatorname{tr} \partial_{i} B^{(0)}=\partial_{i} \operatorname{tr} B^{(0)}=0$ and (3.8) for $k=1$,

$\operatorname{tr}\left(B^{(1)}-\rho^{(1)} \mathbf{1}_{r}\right)=0 \quad$ by Lemma 3.9 and especially,

$\Omega=\Omega^{[2]}-\mathrm{d}\left(\frac{\rho^{(0)}}{z}\right) \cdot \mathbf{1}_{r}=\Omega^{[2]}-\sum_{i=1}^{n} z^{-1} \frac{\partial \rho^{(0)}}{\partial t_{i}} \cdot \mathbf{1}_{r} \mathrm{~d} t_{i}+z^{-2} \rho^{(0)} \cdot \mathbf{1}_{r} \mathrm{~d} z$.

Start with an arbitrary basis $\underline{v}$, consider the function

$$
g:=\frac{1}{r} \sum_{k \geq 2} \frac{-\operatorname{tr} B^{(k)}}{k-1} \cdot z^{k-1} \in z \mathbb{C}\{t, z\},
$$

consider $T:=\mathrm{e}^{g} \cdot \mathbf{1}_{r}$, and $\underline{\widetilde{v}}:=\underline{v} \cdot T .(3.11)$ gives

$$
\widetilde{B}=B+T^{-1} z^{2} \partial_{z} T=B+\left(-\sum_{k \geq 2} \operatorname{tr} B^{(k)} z^{k}\right) \cdot \frac{1}{r} \mathbf{1}_{r}
$$

so $\operatorname{tr} \widetilde{B}^{(k)}=0$ for $k \geq 2, \widetilde{B}^{(1)}=B^{(1)}, \widetilde{B}^{(0)}=B^{(0)}$.

Therefore now suppose $\operatorname{tr}\left(B-z \rho^{(1)} \mathbf{1}_{r}\right)=0$. (3.8) for $k \geq 3$ gives $\operatorname{tr} A_{i}^{(l)}=0$ for $l \geq 2$, because $\operatorname{tr} \partial_{i} B^{(l)}=\partial_{i} \operatorname{tr} B^{(l)}=0$.

Finally, we consider $T=T^{(0)}=\mathrm{e}^{h} \cdot \mathbf{1}_{r}$ for a suitable function $h \in \mathbb{C}\{t\}$. Then $\widetilde{B}=B$, $\widetilde{A}_{i}^{(k)}=A_{i}^{(k)}$ for $k \neq 1$, and $\widetilde{A}_{i}^{(1)}=A_{i}^{(1)}+\partial_{i} h \cdot \mathbf{1}_{r}$. So we need $h \in \mathbb{C}\{t\}$ with $\partial_{i} h=-\frac{1}{r} \operatorname{tr} A_{i}^{(1)}$. Such a function exists because (3.7) for $k=2$ implies $\partial_{i} \operatorname{tr} A_{j}^{(1)}=\partial_{j} \operatorname{tr} A_{i}^{(1)}$. We have obtained a basis $\underline{v}$ with $\operatorname{tr}\left(B-z \rho^{(1)} \mathbf{1}_{r}\right)=0$ and $\operatorname{tr} A_{i}=0$ for all $i$.

\section{$3.3 \quad(T E)$-structures over $\boldsymbol{F}$-manifolds with Euler fields}

The pole part of a $(T)$-structure (or a $(T E)$-structure) over $\mathbb{C} \times M$ along $\{0\} \times M$ induces a Higgs bundle (together with $\mathcal{U}$ ). This is elementary (e.g., [5] or [10]).

Lemma 3.12. Let $(H \rightarrow \mathbb{C} \times M, \nabla)$ be a (T)-structure. Define $K:=\left.H\right|_{\{0\} \times M}$. Then $C:=$ $[z \nabla] \in \Omega^{1}(M$, End $(K))$, more explicitly

$$
C_{X}[a]:=\left[z \nabla_{X} a\right] \quad \text { for } \quad X \in \mathcal{T}_{M}, \quad a \in \mathcal{O}(H),
$$

is a Higgs field, i.e., the endomorphisms $C_{X}, C_{Y}: K \rightarrow K$ for $X, Y \in \mathcal{T}_{M}$ commute.

If $(H \rightarrow \mathbb{C} \times M)$ is a $(T E)$-structure, then its pole part $\mathcal{U}: K \rightarrow K$ commutes with all endomorphisms $C_{X}, X \in \mathcal{T}_{M}$, short: $[C, \mathcal{U}]=0$.

Definition 3.13. The Higgs field of a $(T)$-structure or a $(T E)$-structure $(H \rightarrow \mathbb{C} \times M, \nabla)$ is primitive if there is an open cover $\mathcal{V}$ of $M$ and for any $U \in \mathcal{V}$ a section $\zeta_{U} \in \mathcal{O}\left(\left.K\right|_{U}\right)$ (called a local primitive section) with the property that the map $\mathcal{T}_{U} \ni X \rightarrow C_{X} \zeta_{U} \in \mathcal{O}(K)$ is an isomorphism.

Theorems 3.14 and 3.16 show in two ways that primitivity of a Higgs field is a good condition. Theorem 3.14 was first proved in [11, Theorem 3.3] (but see also [5, Lemma 10]).

Theorem 3.14. $A(T)$-structure $(H \rightarrow \mathbb{C} \times M, \nabla)$ with primitive Higgs field induces a multiplication $\circ$ on $T M$ which makes $M$ an F-manifold. $A(T E)$-structure $(H \rightarrow \mathbb{C} \times M, \nabla)$ with primitive Higgs field induces in addition a vector field $E$ on $M$, which, together with $\circ$, makes $M$ 
an F-manifold with Euler field. The multiplication $\circ$, unit field e and Euler field $E$ (the latter in the case of a (TE)-structure), are defined by

$$
C_{X \circ Y}=C_{X} C_{Y}, \quad C_{e}=\mathrm{Id}, \quad C_{E}=-\mathcal{U},
$$

where $C$ is the Higgs field defined by $\nabla$, and $\mathcal{U}$ is defined in (3.12).

Definition 3.15 recalls the notions of an unfolding and of a universal unfolding of a $(T E)$ structure over a germ of a manifold from [13, Definition 2.3]. It turns out that any $(T E)$ structure over a germ of a manifold with primitive Higgs field is a universal unfolding of itself. Interestingly, we will see in Theorem 8.5 also $(T E)$-structures which are universal unfoldings of themselves, but where the Higgs bundle is only generically primitive. Still in the examples which we consider, the base manifold is an $F$-manifold with Euler field globally.

Malgrange [18] proved that a $(T E)$-structure over a point $t^{0}$ has a universal unfolding with primitive Higgs field if the endomorphism $\mathcal{U}: K_{t^{0}} \rightarrow K_{t^{0}}$ is regular, i.e., it has for each eigenvalue only one Jordan block. A generalization was given by Hertling and Manin [13, Theorem 2.5]. Theorem 3.16 cites in part $(b)$ the generalization. Part $(a)$ is the special case of a $(T E)$ structure with primitive Higgs field. Part $(c)$ is the special case of a $(T E)$-structure over a point, Malgrange's result.

Definition 3.15. Let $\left(H \rightarrow \mathbb{C} \times\left(M, t^{0}\right), \nabla\right)$ be a $(T E)$-structure over a germ $\left(M, t^{0}\right)$ of a manifold.

(a) An unfolding of it is a (TE)-structure $\left(H^{[1]} \rightarrow \mathbb{C} \times\left(M \times \mathbb{C}^{l_{1}},\left(t^{0}, 0\right)\right), \nabla^{[1]}\right)$ over a germ $\left(M \times \mathbb{C}^{l_{1}},\left(t^{0}, 0\right)\right)$ (for some $\left.l_{1} \in \mathbb{N}_{0}\right)$ together with a fixed isomorphism

$$
i^{[1]}:\left.\left(H \rightarrow \mathbb{C} \times\left(M, t^{0}\right), \nabla\right) \rightarrow\left(H^{[1]} \rightarrow \mathbb{C} \times\left(M \times \mathbb{C}^{l_{1}},\left(t^{0}, 0\right)\right), \nabla^{[1]}\right)\right|_{\mathbb{C} \times\left(M \times\{0\},\left(t^{0}, 0\right)\right)} .
$$

(b) One unfolding $\left(H^{[1]} \rightarrow \mathbb{C} \times\left(M \times \mathbb{C}^{l_{1}},\left(t^{0}, 0\right)\right), \nabla^{[1]}, i^{[1]}\right)$ induces a second unfolding $\left(H^{[2]} \rightarrow\right.$ $\left.\mathbb{C} \times\left(M \times \mathbb{C}^{l_{2}},\left(t^{0}, 0\right)\right), \nabla^{[2]}, i^{[2]}\right)$ if there are a holomorphic map germ

$$
\varphi:\left(M \times \mathbb{C}^{l_{2}},\left(t^{0}, 0\right)\right) \rightarrow\left(M \times \mathbb{C}^{l_{1}},\left(t^{0}, 0\right)\right),
$$

which is the identity on $M \times\{0\}$, and an isomorphism $j$ from the second unfolding to the pullback of the first unfolding by $\varphi$ such that

$$
i^{[1]}=\left.j\right|_{\mathbb{C} \times\left(M \times\{0\},\left(t^{0}, 0\right)\right)} \circ i^{[2]} .
$$

(Then $j$ is uniquely determined by $\varphi$ and (3.17).)

(c) An unfolding is universal if it induces any unfolding via a unique map $\varphi$.

By definition of a universal unfolding in part $(c)$, a $(T E)$-structure has (up to canonical isomorphism) at most one universal unfolding, because any two universal unfoldings induce one another by unique maps.

\section{Theorem 3.16.}

(a) ([13, Theorem 2.5]) A (TE)-structure over a germ $\left(M, t^{0}\right)$ with primitive Higgs field is a universal unfolding of itself.

(b) $\left(\left[13\right.\right.$, Theorem 2.5]) Let $\left(H \rightarrow \mathbb{C} \times\left(M, t^{0}\right), \nabla\right)$ be a $(T E)$-structure over a germ $\left(M, t^{0}\right)$ of a manifold. Let $\left(K \rightarrow\left(M, t^{0}\right), C\right)$ be the induced Higgs bundle over $\left(M, t^{0}\right)$. Suppose that a vector $\zeta_{t^{0}} \in K_{t^{0}}$ with the following properties exists: 
(IC) (Injectivity condition) The map $C_{\bullet} \zeta_{t^{0}}: T_{t^{0}} M \rightarrow K_{t^{0}}$ is injective.

$(G C)$ (Generation condition) $\zeta_{t^{0}}$ and its images under iteration of the maps $\left.\mathcal{U}\right|_{t^{0}}: K_{t^{0}} \rightarrow$ $K_{t^{0}}$ and $C_{X}: K_{t^{0}} \rightarrow K_{t^{0}}$ for $X \in T_{t^{0}} M$ generate $K_{t^{0}}$.

Then a universal unfolding of the (TE)-structure over a germ $\left(M \times \mathbb{C}^{l},\left(t^{0}, 0\right)\right)\left(l \in \mathbb{N}_{0}\right.$ suitable) exists. It is unique up to isomorphism. Its Higgs field is primitive.

(c) ([18]) $A(T E)$-structure over a point $t^{0}$ has a universal unfolding with primitive Higgs field if the endomorphism $\left[z^{2} \nabla_{\partial_{z}}\right]=\mathcal{U}: K_{t^{0}} \rightarrow K_{t^{0}}$ is regular, i.e., it has for each eigenvalue only one Jordan block. In that case, the germ of the F-manifold with Euler field which underlies the universal unfolding, is by definition (Definition 2.4) regular.

\section{Remarks 3.17.}

(i) As said above, the parts $(a)$ and $(c)$ are special cases of part $(b)$.

(ii) A germ $\left(\left(M, t^{0}\right), \circ, e, E\right)$ of a regular $F$-manifold is uniquely determined by the regular endomorphism $\left.E \circ\right|_{t^{0}}: T_{t^{0}} \rightarrow T_{t^{0}}$ (Theorem 2.5).

(iii) Consider the germ $(M, 0)=\left(\mathbb{C}^{2}, 0\right)$ of a 2-dimensional $F$-manifold with Euler field $E$ in Theorem 2.2. It is regular if and only if $\left.E \circ\right|_{t=0} \notin\{\lambda \mathrm{id} \mid \lambda \in \mathbb{C}\}$. In the semisimple case (Theorem 2.2(a)) this holds if and only if $c_{1} \neq c_{2}$. In the cases $I_{2}(m)(m \geq 3)$ it does not hold. In the case of $\mathcal{N}_{2}$ with $E=t_{1} \partial_{1}+g\left(t_{2}\right) \partial_{2}$ it holds if and only if $g(0) \neq 0$. See also Remark 2.6(ii).

(iv) Theorem 3.16(c) implies that a $(T E)$-structure with primitive Higgs field over a germ $\left(M, t^{0}\right)$ of a regular $F$-manifold with Euler field is determined up to gauge isomorphism by the restriction of the $(T E)$-structure to $t^{0}$.

$(v)$ Lemma 3.6, Definition 3.8, Lemmata 3.9-3.12, Definition 3.13, Theorem 3.14 and Definition 3.15 hold or make sense also for formal $(T)$-structures or $(T E)$-structures. However, the proof of Theorem 3.16 used in an essential way holomorphic (TE)-structures. We do not know whether Theorem 3.16 holds also for formal (TE)-structures.

\subsection{Birkhoff normal form}

Definition 3.18. Let $(H \rightarrow \mathbb{C} \times M, \nabla)$ be a $(T E)$-structure over a manifold $M$ with coordinates $t=\left(t_{1}, \ldots, t_{n}\right)$. A Birkhoff normal form consists of a basis $\underline{v}$ of $H$ and associated matrices $A_{1}, \ldots, A_{n}, B$ as in (3.2) such that

$$
A_{1}^{(k)}=\cdots=A_{n}^{(k)}=0 \quad \text { for } \quad k \geq 1, \quad B^{(k)}=0 \quad \text { for } \quad k \geq 2, \quad \partial_{i} B^{(1)}=0 .
$$

\section{Remarks 3.19.}

(i) Such a basis defines an extension of the $(T E)$-structure to a pure $(T L E)$-structure. Then it is a basis of the $(T L E)$-structure whose restriction to $\{\infty\} \times M$ is flat with respect to the residual connection (that is just the restriction of the connection $\nabla$ of the underlying $(T L)$-structure to $\left.\left.H\right|_{\{\infty\} \times M}\right)$. Then the conditions (3.7) and (3.8) boil down to

$$
\begin{array}{ll}
0=\left[A_{i}^{(0)}, A_{j}^{(0)}\right], & \partial_{i} A_{j}^{(0)}=\partial_{j} A_{i}^{(0)}, \\
0=\left[A_{i}^{(0)}, B^{(0)}\right], & \partial_{i} B^{(0)}+A_{i}^{(0)}+\left[A_{i}^{(0)}, B^{(1)}\right], \quad 0=\partial_{i} B^{(1)} .
\end{array}
$$

Such a basis is relevant for the construction of Frobenius manifolds (see, e.g., [7]). 
(ii) Vice versa, if the (TE)-structure has an extension to a pure (TLE)-structure, then a basis $\underline{v}$ of the $(T L E)$-structure exists whose restriction to $\{\infty\} \times M$ is flat with respect to the residual connection. Then this basis $\underline{v}$ and the associated matrices form a Birkhoff normal form.

(iii) A Birkhoff normal form does not always exist. But if a Birkhoff normal form of the restriction of a $(T E)$-structure over $M$ to a point $t^{0} \in M$ exists, it extends to a Birkhoff normal form of the $(T E)$-structure over the germ $\left(M, t^{0}\right)$ [21, Chapter VI, Theorem 2.1] (or $[7$, Theorem 5.1(c)]).

(iv) The problem whether a $(T E)$-structure over a point has an extension to a pure $(T L E)$ structure is a special case of the Birkhoff problem, which itself is a special case of the Riemann-Hilbert-Birkhoff problem. The book [1] and Chapter IV in [21] are devoted to these problems and results on them.

Here the following two results on the Birkhoff problem will be useful. However, we will use part $(a)$ only in the case of a $(T E)$-structure over a point $t^{0}$ with a logarithmic pole at $z=0$, in which case it is trivial.

Theorem 3.20. Let $\left(H \rightarrow \mathbb{C} \times\left\{t^{0}\right\}, \nabla\right)$ be a (TE)-structure over a point $t^{0}$.

(a) (Plemely, [21, Chapter IV, Corollary 2.6(1)]) If the monodromy is semisimple, the (TE)structure has an extension to a pure (TLE)-structure.

(b) (Bolibroukh and Kostov, [21, Chapter IV, Corollary 2.6(3)]) The germ $\mathcal{O}(H)_{0} \otimes_{\mathbb{C}\{z\}}$ $\mathbb{C}\{z\}\left[z^{-1}\right]$ is a $\mathbb{C}\{z\}\left[z^{-1}\right]$-vector space of dimension $r=\mathrm{rk} H \in \mathbb{N}$ on which $\nabla$ acts.

If no $\mathbb{C}\{z\}\left[z^{-1}\right]$ sub vector space of dimension in $\{1, \ldots, r-1\}$ exists which is $\nabla$-invariant, then the (TE)-structure has an extension to a pure (TLE)-structure.

\subsection{Regular singular ( $T E$ )-structures}

A $(T E)$-structure over a point $t^{0}$ is regular singular if all its holomorphic sections have moderate growth near 0. A good tool to treat this situation are special sections of moderate growth, the elementary sections. Definition 3.21 explains them and other basic notations. We work with a simply connected manifold $M$, so that the only monodromy is the monodromy along closed paths in the punctured $z$-plane going around 0 . One important case is the case of a germ $\left(M, t^{0}\right)$ of a manifold. The most important case is the case of a point, $M=\left\{t^{0}\right\}$.

Definition 3.21. Let $(H \rightarrow \mathbb{C} \times M, \nabla)$ be a $(T E)$-structure of rank $r=\operatorname{rk} H \in \mathbb{N}$ over a simply connected manifold $M$. We associate the following data to it.

(a) $H^{\prime}:=\left.H\right|_{\mathbb{C}^{*} \times M}$ is the flat bundle on $\mathbb{C}^{*} \times M . H^{\infty}$ denotes the $\mathbb{C}$-vector space (of dimension $r$ ) of global flat multivalued sections on $H^{\prime}$. Let the endomorphism $M^{\text {mon }}: H^{\infty} \rightarrow$ $H^{\infty}$ be the monodromy on it with semisimple part $M_{s}^{\text {mon }}$, unipotent part $M_{u}^{\text {mon }}$ (with $\left.M_{s}^{\text {mon }} M_{u}^{\text {mon }}=M_{u}^{\text {mon }} M_{s}^{\text {mon }}\right)$, nilpotent part $N^{\text {mon }}:=\log M_{u}^{\text {mon }}$ so that $M_{u}^{\text {mon }}=\mathrm{e}^{N^{\text {mon }}}$, and with eigenvalues in the finite set $\operatorname{Eig}\left(M^{\text {mon }}\right) \subset \mathbb{C}$. For $\lambda \in \mathbb{C}$, let

$$
H_{\lambda}^{\infty}:=\operatorname{ker}\left(M_{s}^{\mathrm{mon}}-\lambda \mathrm{id}: H^{\infty} \rightarrow H^{\infty}\right)
$$

be the generalized eigenspace in $H^{\infty}$ of the monodromy with eigenvalue $\lambda$. It is not $\{0\}$ if and only if $\lambda \in \operatorname{Eig}\left(M^{\mathrm{mon}}\right)$.

(b) For $\alpha \in \mathbb{C}$, define the finite dimensional $\mathbb{C}$-vector space $C^{\alpha}$ of the following global sections of $H^{\prime}$,

$$
C^{\alpha}:=\left\{\sigma \in \mathcal{O}\left(H^{\prime}\right)\left(\mathbb{C}^{*}\right) \mid\left(\nabla_{z \partial_{z}}-\alpha \mathrm{id}\right)^{r}(\sigma)=0, \nabla_{\partial_{i}}(\sigma)=0\right\}
$$


(where $t=\left(t_{1}, \ldots, t_{n}\right)$ are local coordinate and $\partial_{i}$ are the coordinate vector fields). Observe $z^{k} \cdot C^{\alpha}=C^{\alpha+k}$ for $k \in \mathbb{Z}$. For each $\alpha$ the map

$$
\begin{aligned}
s(\cdot, \alpha): H_{\mathrm{e}^{-2 \pi \mathrm{i} \alpha}}^{\infty} & \rightarrow C^{\alpha}, \\
A & \mapsto s(A, \alpha):=z^{\alpha} \cdot \mathrm{e}^{-\log z \cdot N^{\operatorname{mon}} / 2 \pi \mathrm{i}} A(\log z),
\end{aligned}
$$

is an isomorphism. So, $C^{\alpha} \neq\{0\}$ if and only if $\mathrm{e}^{-2 \pi \mathrm{i} \alpha} \in \operatorname{Eig}\left(M^{\mathrm{mon}}\right)$. The sections $s(A, \alpha)$ are called elementary sections.

(c) A holomorphic section $\sigma$ of $\left.H^{\prime}\right|_{\left(U_{1} \backslash\{0\}\right) \times U_{2}}$ for $U_{1} \subset \mathbb{C}$ a neighborhood of $0 \in \mathbb{C}$ and $U_{2} \subset M$ open in $M$ can be written uniquely as an (in general infinite) sum of elementary sections es $(\sigma, \alpha) \in \mathcal{O}_{U_{2}} \cdot C^{\alpha}$ with coefficients in $\mathcal{O}_{U_{2}}$,

$$
\sigma=\sum_{\alpha: \mathrm{e}^{-2 \pi \mathrm{i} \alpha} \in \operatorname{Eig}\left(M^{\mathrm{mon}}\right)} \operatorname{es}(\sigma, \alpha) .
$$

In order to see this, choose numbers $\alpha_{j} \in \mathbb{C}$ and elementary sections $s_{j} \in C^{\alpha_{j}}$ for $j \in$ $\{1, \ldots, r\}$ such that $s_{1}, \ldots, s_{r}$ form a global basis of $H^{\prime}$. Then

$$
\sigma=\sum_{j=1}^{r} a_{j} s_{j} \quad \text { with } \quad a_{j}=a_{j}(z, t)=\sum_{k=-\infty}^{\infty} a_{k j}(t) z^{k} \in \mathcal{O}_{\left(U_{1} \backslash\{0\}\right) \times U_{2}} .
$$

Here (3.20) is the expansion of $a_{j}$ as a Laurent series in $z$ with holomorphic coefficients $a_{k j} \in \mathcal{O}_{U_{2}}$ in $t$. Then

$$
\operatorname{es}(\sigma, \alpha)(z, t)=\sum_{j: \alpha-\alpha_{j} \in \mathbb{Z}} a_{\alpha-\alpha_{j}, j}(t) z^{\alpha-\alpha_{j}} s_{j}
$$

(d) A holomorphic section $\sigma$ as in $(c)$ has moderate growth if a bound $b \in \mathbb{R}$ with es $(\sigma, \alpha)=0$ for all $\alpha$ with $\operatorname{Re}(\alpha)<b$ exists. The sheaf $\mathcal{V}^{>-\infty}$ on $\mathbb{C} \times M$ of all sections of moderate growth is

$$
\mathcal{V}^{>-\infty}:=\bigoplus_{\alpha:-1<\operatorname{Re}(\alpha) \leq 0} \mathcal{O}_{\mathbb{C} \times M}\left[z^{-1}\right] \cdot C^{\alpha} .
$$

The Kashiwara-Malgrange $V$-filtration is given by the locally free subsheaves for $r \in \mathbb{R}$,

$$
\mathcal{V}^{r}:=\bigoplus_{\alpha: \operatorname{Re}(\alpha) \in[r, r+1[} \mathcal{O}_{\mathbb{C} \times M} \cdot C^{\alpha}
$$

\section{Definition 3.22.}

(a) A (TE)-structure $(H \rightarrow \mathbb{C} \times M, \nabla)$ over a simply connected manifold $M$ is regular singular if $\mathcal{O}(H) \subset \mathcal{V}^{>-\infty}$, so if all its holomorphic sections have moderate growth near 0 .

(b) A (TE)-structure $(H \rightarrow \mathbb{C} \times M, \nabla)$ over a simply connected manifold $M$ is logarithmic if it has a basis $\underline{v}$ whose connection 1 -form $\Omega$ has a logarithmic pole along $\{0\} \times M$ (then this holds for any basis). In the notations of (3.2)-(3.4) that means $A_{i}^{(0)}=B^{(0)}=0$. Then the restriction of $\nabla$ to $K:=\left.H\right|_{\{0\} \times M}$ is well-defined. It is called the residual connection $\nabla^{\text {res }}$. The residue endomorphism is $\operatorname{Res}_{0}=\left[\nabla_{z \partial_{z}}\right]: K \rightarrow K$.

Theorem 3.23 (well known, e.g., [9, Theorems 7.10 and 8.7]). Let $(H \rightarrow \mathbb{C} \times M, \nabla)$ with $\left.H\right|_{\mathbb{C}^{*} \times M}=H^{\prime}$ be a logarithmic (TE)-structure over a simply connected manifold. 
(a) The bundle $H$ has a global basis which consists of elementary sections $s_{j} \in C^{\alpha_{j}}, j \in$ $\{1, \ldots$, rk $H\}$, for some $\alpha_{j} \in \mathbb{C}$. Especially, $(\mathcal{O}(H), \nabla)=\varphi_{t^{0}}^{*}\left(\mathcal{O}\left(\left.H\right|_{\mathbb{C} \times\left\{t^{0}\right\}}\right), \nabla\right)$ for any $t^{0} \in M$, where $\varphi_{t^{0}}: M \rightarrow\left\{t^{0}\right\}$ is the projection. So it is just the pull back of a logarithmic (TE)-structure over a point. Especially, it is a regular singular (TE)-structure.

(b) The residual connection $\nabla^{\mathrm{res}}$ is flat. In the notations (3.2)-(3.4), its connection 1-form is $\sum_{i=1}^{n} A_{i}^{(1)} \mathrm{d} t_{i}$. The residue endomorphism Res is $\nabla^{\mathrm{res}}-$ flat. In the notations $(3.2)-(3.4)$, it is given by $B^{(1)}$.

(c) The endomorphism $\mathrm{e}^{-2 \pi \operatorname{Res}_{0}}: K \rightarrow K$ has the same eigenvalues as the monodromy $M^{\text {mon }}$, but it might have a simpler Jordan block structure. If no eigenvalues of $\operatorname{Res}_{0}$ differ by a nonzero integer (nonresonance condition) then $\mathrm{e}^{-2 \pi \operatorname{Res}_{0}}$ has the same Jordan block structure as the monodromy $M^{\text {mon }}$.

\section{Remarks 3.24.}

(i) Part $(a)$ of Theorem 3.23 implies that a logarithmic $(T E)$-structure over a simply connected manifold $M$ is the pull back $\varphi^{*}\left(\left.(H, \nabla)\right|_{\mathbb{C} \times\left\{t^{0}\right\}}\right)$ of its restriction to $t^{0}$ for any $t^{0} \in M$.

(ii) In the case of a regular singular $(T E)$-structure over a simply connected manifold $M$, one can choose elementary sections $s_{j} \in C^{\alpha_{j}}, j \in\{1, \ldots, \mathrm{rk} H\}$, for some $\alpha_{j} \in \mathbb{C}$, such that they form a basis of $H^{*}$ and such the extension to $\{0\} \times M$ which they define, is a logarithmic $(T E)$-structure. Then the base change from any local basis of $H$ to the basis $\left(s_{1}, \ldots, s_{\mathrm{rk} H}\right)$ of this new $(T E)$-structure is meromorphic, so the two $(T E)$-structures give the same meromorphic bundle. This observation fits to the usual definition of meromorphic bundle with regular singular pole.

(iii) The property of a section to have moderate growth, is invariant under pull back. Therefore also the property of a (TE)-structure to be regular singular is invariant under pull back.

\subsection{Marked ( $T E$ )-structures and moduli spaces for them}

It is easy to give a $(T E)$-structure $(H \rightarrow \mathbb{C} \times M, \nabla)$ with nontrivial Higgs field and which is thus not the pull back of the $(T E)$-structure over a point, such that nevertheless the $(T E)$ structures over all points $t^{0} \in M$ are isomorphic as abstract $(T E)$-structures. Examples are given in Remark 7.1(ii). The existence of such $(T E)$-structures obstructs the construction of nice Hausdorff moduli spaces for (TE)-structures up to isomorphism. The notion of a marked (TE)structure hopefully remedies this. However, in the moment, we have only results in the regular singular cases. Definition 3.25 gives the notion of a marked (TE)-structure. Definition 3.26 defines good families of marked regular singular (TE)-structures. Definition 3.28 defines a functor for such families. Theorem 3.29 states that this functor is represented by a complex space. It builds on results in [15, Chapter 7]. Several remarks discuss what is missing in the other cases and what more we have in the regular singular rank 2 case, thanks to Theorems 6.3, 6.7 and 8.5.

\section{Definition 3.25.}

(a) A reference pair $\left(H^{\mathrm{ref}, \infty}, M^{\mathrm{ref}}\right)$ consists of a finite dimensional (reference) $\mathbb{C}$-vector space $H^{\text {ref, } \infty}$ together with an automorphism $M^{\text {ref }}$ of it.

(b) Let $M$ be a simply connected manifold. A marking on a $(T E)$-structure $(H \rightarrow \mathbb{C} \times M, \nabla)$ is an isomorphism $\psi:\left(H^{\infty}, M^{\text {mon }}\right) \rightarrow\left(H^{\text {ref, } \infty}, M^{\text {ref }}\right)$. Here $H^{\infty}$ is (as in Definition 3.16) the space of global flat multivalued sections on the flat bundle $H^{\prime}:=\left.H\right|_{\mathbb{C}^{*} \times M}$, and $M^{\text {mon }}$ is its monodromy. ( $\left.H^{\mathrm{ref}, \infty}, M^{\mathrm{ref}}\right)$ is a reference pair. The isomorphism $\psi$ of pairs means an isomorphism $\psi: H^{\infty} \rightarrow H^{\text {ref, } \infty}$ with $\psi \circ M^{\text {mon }}=M^{\text {ref }} \circ \psi$. A marked $(T E)$-structure is a $(T E)$-structure with a marking. 
(c) An isomorphism between two marked (TE)-structures $\left(\left(H^{(1)}, \nabla^{(1)}\right), \psi^{(1)}\right)$ and $\left(\left(H^{(2)}, \nabla^{(2)}\right), \psi^{(2)}\right)$ over the same base space $M^{(1)}=M^{(2)}$ and with the same reference pair $\left(H^{\text {ref, } \infty}, M^{\text {ref }}\right)$ is a gauge isomorphism $\varphi$ between the unmarked $(T E)$-structures such that the induced isomorphism $\varphi^{\infty}: H^{(1), \infty} \rightarrow H^{(2), \infty}$ is compatible with the marking,

$$
\psi^{(2)} \circ \varphi^{\infty}=\psi^{(1)} \text {. }
$$

(d) $\operatorname{Set}^{\left(H^{\mathrm{ref}, \infty}, M^{\mathrm{ref}}\right)}$ denotes the set of marked $(T E)$-structures over a point with the same reference pair $\left(H^{\mathrm{ref}, \infty}, M^{\mathrm{ref}}\right)$. Furthermore, $\operatorname{Set}^{\left(H^{\mathrm{ref}, \infty}, M^{\mathrm{ref}}\right), \mathrm{reg}} \subset \operatorname{Set}^{\left(H^{\mathrm{ref}, \infty}, M^{\mathrm{ref}}\right)}$ denotes the subset of marked regular singular $(T E)$-structures over a point with the same reference pair $\left(H^{\mathrm{ref}, \infty}, M^{\mathrm{ref}}\right)$.

We hope that $\operatorname{Set}^{\left(H^{\mathrm{ref}, \infty}, M^{\mathrm{ref}}\right)}$ carries for any reference pair $\left(H^{\mathrm{ref}, \infty}, M^{\mathrm{ref}}\right)$ a natural structure as a complex space. Theorem 3.29 says that this holds for $\operatorname{Set}^{\left(H^{\mathrm{ref}, \infty}, M^{\mathrm{ref}}\right), \mathrm{reg}}$ and that this space represents a functor of good families of marked regular singular $(T E)$-structures. Definition 3.26 gives a notion of a family of marked (TE)-structures and the notion of a good family of marked regular singular (TE)-structures.

Definition 3.26. Let $X$ be a complex space. Let $t^{0}$ be an abstract point and $\varphi: X \rightarrow\left\{t^{0}\right\}$ be the projection. Let $\left(H^{\text {ref, } \infty}, M^{\text {ref }}\right)$ be a reference pair. Let $\left(H^{\text {ref,* }}, \nabla^{\text {ref }}\right)$ be a flat bundle on $\mathbb{C}^{*} \times\left\{t^{0}\right\}$ with monodromy $M^{\text {ref }}$ and whose space of global flat multivalued sections is identified with $H^{\text {ref, } \infty}$. Let $i: \mathbb{C}^{*} \times X \hookrightarrow \mathbb{C} \times X$ be the inclusion.

(a) A family of marked (TE)-structures over $X$ is a pair $(H, \psi)$ with the following properties:

( $i) H$ is a holomorphic vector bundle on $\mathbb{C} \times X$, i.e., the linear space associated to a locally

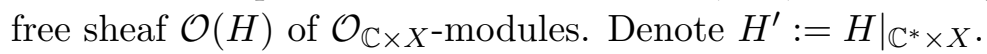

(ii) $\psi$ is an isomorphism $\psi: H^{\prime} \rightarrow \varphi^{*} H^{\text {ref,* }}$ such that the restriction of the induced flat connection on $H^{\prime}$ to $\mathbb{C}^{*} \times\{x\}$ for any $x \in X$ makes $\left.H\right|_{\mathbb{C} \times\{x\}}$ into a $(T E)$-structure over the point $x$, i.e., the connection has a pole of order $\leq 2$ on holomorphic sections of $\left.H\right|_{\mathbb{C} \times\{x\}}$.

(b) Consider a family $(H, \psi)$ of marked regular singular $(T E)$-structures over $X$. The marking $\psi$ induces for each $x \in X$ canonical isomorphisms

$$
\begin{array}{lll}
\psi: & H^{\infty}(x) \rightarrow H^{\text {ref }, \infty}, \\
\psi: & C^{\alpha}(x) \rightarrow C^{\text {ref, }, \alpha} \quad\left(\alpha \in \mathbb{C} \text { with } \mathrm{e}^{-2 \pi \mathrm{i} \alpha} \in \operatorname{Eig}\left(M^{\mathrm{ref}}\right)\right), \\
\psi: & V^{r}(x) \rightarrow V^{\mathrm{ref}, r} \quad(r \in \mathbb{R}),
\end{array}
$$

where $H^{\infty}(x), C^{\alpha}(x), V^{r}(x)$ and $C^{\mathrm{ref}, \alpha}, V^{\mathrm{ref}, r}$ are defined for the (TE)-structure over $x$ respectively for $\left(H^{\text {ref,* }}, \nabla\right)$ as in Definition 3.21.

The family $(H, \psi)$ is called good if some $r \in \mathbb{R}$ and some $N \in \mathbb{N}$ exist which satisfy

$$
\begin{array}{lll}
\mathcal{O}\left(\left.H\right|_{\mathbb{C} \times\{x\}}\right)_{0} \supset V^{r}(x) \quad \text { for any } & x \in X, \\
\operatorname{dim}_{\mathbb{C}} \mathcal{O}\left(\left.H\right|_{\mathbb{C} \times\{x\}}\right)_{0} / V^{r}(x)=N \quad \text { for any } & x \in X .
\end{array}
$$

\section{Remarks 3.27.}

(i) The notion of a family of marked $(T E)$-structures is too weak. For example, it contains the following pathological family of logarithmic $(T E)$-structures of rank 1 over $X:=\mathbb{C}$ (with coordinate $t$ ) and with trivial monodromy. Write $s_{0} \in C^{0}$ for a generating flat section. Define $H$ by

$$
\mathcal{O}(H)=\mathcal{O}_{\mathbb{C} \times X} \cdot\left(t+z^{l}\right) s_{0} \quad \text { for some } \quad l \in \mathbb{N} .
$$


The marked (TE)-structures over all points $t \in \mathbb{C}^{*} \subset X=\mathbb{C}$ are isomorphic and even equal, the one over $t=0$ is different. The dimension $\mathcal{O}\left(\left.H\right|_{\mathbb{C} \times\{t\}}\right) / V^{l}(t)$ is equal to $l$ for $t \in \mathbb{C}^{*}$ and equal to 0 for $t=0$. Therefore this family is not good in the sense of Definition 3.26(b). Also, $z \nabla_{\partial_{z}}\left(t+z^{l}\right) s_{0}=l z^{l} s_{0}$ is not a section in $\mathcal{O}(H)$, although for each fixed $t \in X$, the restriction to $\mathbb{C} \times\{t\}$ is a section in $\mathcal{O}\left(\left.H\right|_{\mathbb{C} \times\{t\}}\right)$.

(ii) Theorem 3.29 gives evidence that the notion of a good family of marked regular singular $(T E)$-structures is useful. However, it is not clear a priori whether any regular singular $(T E)$-structure $(H \rightarrow \mathbb{C} \times M, \nabla)$ over a simply connected manifold $M$ is a good family of marked regular singular $(T E)$-structures over $X=M$. A marking can be imposed as $M$ is simply connected. Though the condition (3.22) is not clear a priori. Theorem 8.5 will show this for regular singular rank $2(T E)$-structures. It builds on Theorems 6.3 and 6.7 which show this for regular singular rank $2(T E)$-structures over $M=\mathbb{C}$.

(iii) For not regular singular $(T E)$-structures, we do not see an easy replacement of condition (3.22). Is the condition $z^{2} \nabla_{\partial_{z}} \mathcal{O}(H) \subset \mathcal{O}(H)$ useful?

Definition 3.28. Fix a reference pair $\left(H^{\mathrm{ref}, \infty}, M^{\mathrm{ref}}\right)$.

(a) Define the functor $\mathcal{M}^{\left(H^{\mathrm{ref}, \infty}, M^{\mathrm{ref}}\right) \text {,reg }}$ from the category of complex spaces to the category of sets by

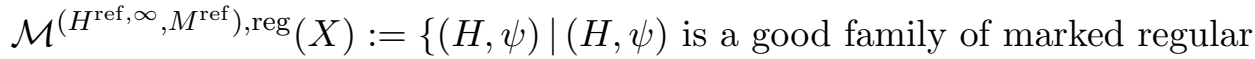

$$
\begin{aligned}
& \text { singular }(T E) \text {-structures over } X\} \text {, }
\end{aligned}
$$

and, for any morphism $f: Y \rightarrow X$ of complex spaces and any element $(H, \psi)$ of $\mathcal{M}^{\left(H^{\mathrm{ref}, \infty}, M^{\mathrm{ref}}\right), \mathrm{reg}}(X)$, define $\mathcal{M}^{\left(H^{\mathrm{ref}, \infty}, M^{\mathrm{ref}}\right), \mathrm{reg}}(f)(H, \psi):=f^{*}(H, \psi)$.

(b) Choose $r \in \mathbb{R}$ and $N \in \mathbb{N}$. Define the functor $\mathcal{M}^{\left(H^{\mathrm{ref}, \infty}, M^{\mathrm{ref}}\right), r, N}$ from the category of complex spaces to the category of sets by

$$
\begin{aligned}
& \mathcal{M}^{\left(H^{\mathrm{ref}, \infty}, M^{\mathrm{ref}}\right), r, N}(X):=\{(H, \psi) \mid(H, \psi) \text { is a good family of marked regular } \\
& \text { singular }(T E) \text {-structures over } X \text { which satisfies (3.21) } \\
&\text { and }(3.22) \text { for the given } r \text { and } N\},
\end{aligned}
$$

and, for any morphism $f: Y \rightarrow X$ of complex spaces and any element $(H, \psi)$ of $\mathcal{M}^{\left(H^{\mathrm{ref}, \infty}, M^{\mathrm{ref}}\right), r, N}(X)$, define $\mathcal{M}^{\left(H^{\mathrm{ref}, \infty}, M^{\mathrm{ref}}\right), r, N}(f)(H, \psi):=f^{*}(H, \psi)$.

Theorem 3.29. The functors $\mathcal{M}^{\left(H^{\mathrm{ref}, \infty}, M^{\mathrm{ref}}\right), \mathrm{reg}}$ and $\mathcal{M}^{\left(H^{\mathrm{ref}, \infty}, M^{\mathrm{ref}}\right), r, N}$ are represented by complex spaces, which are called $M^{\left(H^{\mathrm{ref}, \infty}, M^{\mathrm{ref}}\right), \mathrm{reg}}$ and $M^{\left(H^{\mathrm{ref}, \infty}, M^{\mathrm{ref}}\right), r, N}$. In the case of $\mathcal{M}^{\left(H^{\mathrm{ref}, \infty}, M^{\mathrm{ref}}\right), r, N}$, the complex space has even the structure of a projective algebraic variety. As sets $M^{\left(H^{\mathrm{ref}, \infty}, M^{\mathrm{ref}}\right), \mathrm{reg}}=\operatorname{Set}^{\left(H^{\mathrm{ref}, \infty}, M^{\mathrm{ref}}\right), \mathrm{reg}}$.

Proof. The proof for $\mathcal{M}^{\left(H^{\mathrm{ref}, \infty}, M^{\mathrm{ref}}\right), r, N}$ can be copied from the proof of Theorem 7.3 in [15]. Here it is relevant that $r$ and $N$ with (3.21) and (3.22) imply the existence of an $r_{2} \in \mathbb{R}$ with $r_{2}<r$ and

$$
V^{r_{2}}(x) \supset \mathcal{O}\left(\left.H\right|_{\mathbb{C} \times\{x\}}\right)_{0} \quad \text { for any } \quad x \in X .
$$

In [15], (TERP)-structures are considered. (3.21) and (3.23) are demanded there. (3.22) is not demanded there explicitly, but it follows from the properties of the pairing there, and this is used in Lemma 7.2 in [15]. The additional conditions of (TERP)-structures are not essential for the arguments in the proof of Lemma 7.2 and Theorem 7.3 in [15]. Therefore these proofs apply also here and give the statements for $\mathcal{M}^{\left(H^{\mathrm{ref}, \infty}, M^{\mathrm{ref}}\right), r, N}$. 
Let us call $(r, N) \in \mathbb{R} \times \mathbb{N}$ and $(\widetilde{r}, \tilde{N}) \in \mathbb{R} \times \mathbb{N}$ compatible if $n \in \mathbb{Z}$ with $(\widetilde{r}, \tilde{N})=(r+n$, $\left.N+n \cdot \operatorname{dim} H^{\mathrm{ref}, \infty}\right)$ exists. In the case $n>0, \mathcal{M}^{\left(H^{\mathrm{ref}, \infty}, M^{\mathrm{ref}}\right), \widetilde{r}, \widetilde{N}}$ is a union of $\mathcal{M}^{\left(H^{\mathrm{ref}, \infty}, M^{\mathrm{ref}}\right), r, N}$ and additional irreducible components. Thus for fixed $(r, N)$ the union

$$
\bigcup_{n \in \mathbb{N}} M^{\left(H^{\mathrm{ref}, \infty}, M^{\mathrm{ref}}\right), r+n, N+n \cdot \operatorname{dim} H^{\mathrm{ref}, \infty}}
$$

is a complex space with in general countably many irreducible (and compact) components.

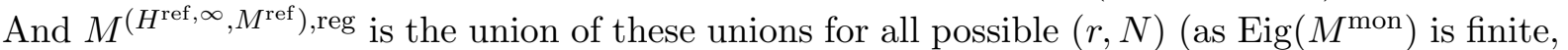
in each interval of length 1 , only finitely many $r$ are relevant).

\section{Remarks 3.30.}

(i) For each reference pair $\left(H^{\text {ref, } \infty}, M^{\text {ref }}\right)$ with $\operatorname{dim} H^{\text {ref }, \infty}=2$, the representing complex space

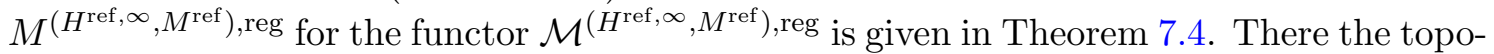
logical components are unions $\bigcup_{n \in \mathbb{N}} M^{\left(H^{\mathrm{ref}, \infty}, M^{\mathrm{ref}}\right), r+n, N+n \cdot \operatorname{dim} H^{\mathrm{ref}, \infty}}$ and have countably many irreducible components which are either isomorphic to $\mathbb{P}^{1}$ or to the Hirzebruch surface $\mathbb{F}_{2}$ or to the variety $\widetilde{\mathbb{F}}_{2}$ obtained by blowing down the $(-2)$-curve in $\mathbb{F}_{2}$. The space $M^{\left(H^{\mathrm{ref}, \infty}, M^{\mathrm{ref}}\right), \text { reg }}$ is a union of countably many copies of one topological component.

(ii) Corollary 7.3 says that any marked rank 2 regular singular $(T E)$-structure $(H \rightarrow \mathbb{C} \times$ $M, \nabla, \psi)$ with reference pair $\left(H^{\text {ref, } \infty}, M^{\text {ref }}\right)$ is a good family of marked regular singular $(T E)$-structures. Therefore and because of Theorem 3.29, such a $(T E)$-structure is induced by a morphism $\varphi: M \rightarrow M^{\left(H^{\mathrm{ref}, \infty}, M^{\mathrm{ref}}\right) \text {,reg }}$. This is crucial for the usefulness of the space $M^{\left(H^{\mathrm{ref}, \infty}, M^{\mathrm{ref}}\right), \text { reg }}$. We hope that Corollary 7.3 and this implication are also true for higher rank regular singular $(T E)$-structures.

\section{Rank 2 (TE)-structures over a point}

Here we will classify the rank $2(T E)$-structures over a point.

\subsection{Separation into 4 cases}

They separate naturally into 4 cases.

Definition 4.1. Let $(H \rightarrow \mathbb{C}, \nabla)$ be a rank $2(T E)$-structure over a point $t^{0}=0$. Its formal invariants $\delta^{(0)}, \rho^{(0)}, \delta^{(1)}, \rho^{(1)}$ from Lemma 3.9 are complex numbers. The eigenvalues of $-\mathcal{U}$ are called $u_{1}, u_{2} \in \mathbb{C}$. They are given by $\left(x-u_{1}\right)\left(x-u_{2}\right)=x^{2}+2 \rho^{(0)} x+\delta^{(0)}$. We separate four cases:

(Sem) $\mathcal{U}$ has two different eigenvalues $-u_{1}$ and $-u_{2} \in \mathbb{C}$, i.e., $0 \neq \delta^{(0)}-\left(\rho^{(0)}\right)^{2}$.

(Bra) $\mathcal{U}$ has only one eigenvalue (which is then $\rho^{(0)}$ ) and one $2 \times 2$ Jordan block, and $\delta^{(1)}-$ $2 \rho^{(0)} \rho^{(1)} \neq 0$.

(Reg) $\mathcal{U}$ has only one eigenvalue (which is then $\rho^{(0)}$ ) and one $2 \times 2$ Jordan block, and $\delta^{(1)}-$ $2 \rho^{(0)} \rho^{(1)}=0$.

$(\log ) \mathcal{U}=\rho^{(0)} \cdot \mathrm{id}$

Here (Sem) stands for semisimple, (Bra) for branched, (Reg) for regular singular and (Log) for logarithmic. 
Remark 4.2. Rank 2 (TE)-structures over a point are richer than the germs of mermorphic rank 2 vector bundles with a pole of order 2 . Though the four types above are closely related to the formal classification of the latter ones by their slopes (the notion of slopes is developed for example in [19, Section 5]). In rank 2, three slopes are possible, slope 1, slope $\frac{1}{2}$ and slope 0 . Slope 1 corresponds to the type (Sem), slope $\frac{1}{2}$ to type (Bra), and slope 0 to the types (Reg) and $(\log )$.

First we will treat the semisimple case (Sem). Then the cases (Bra), (Reg) and (Log) will be considered together. Lemma 4.9 will justify the names (Bra) and (Reg). Finally, the three cases (Bra), (Reg) and (Log) will be treated one after the other. The following lemma gives some first information. Its proof is straightforward.

Lemma 4.3. Let $(H \rightarrow \mathbb{C}, \nabla)$ be a rank $2(T E)$-structure over a point. Denote by $(\widetilde{H} \rightarrow$ $\mathbb{C}, \widetilde{\nabla})$ the $(T E)$-structure with trace free pole part with $(\mathcal{O}(\widetilde{H}), \widetilde{\nabla})=(\mathcal{O}(H), \nabla) \otimes \mathcal{E}^{\rho^{(0)} / z}$ from Lemma $3.10(b)$ (called $\left(H^{[2]} \rightarrow \mathbb{C}, \nabla^{[2]}\right)$ there), and denote its invariants from Lemma 3.9 by $\widetilde{\mathcal{U}}, \widetilde{\delta}^{(0)}, \widetilde{\rho}^{(0)}, \widetilde{\delta}^{(1)}, \widetilde{\rho}^{(1)}$. Then

$$
\begin{array}{ll}
\widetilde{\mathcal{U}}=\mathcal{U}-\rho^{(0)} \mathrm{id}, & \\
\widetilde{\delta}^{(0)}=\delta^{(0)}-\left(\rho^{(0)}\right)^{2}, & \widetilde{\rho}^{(0)}=0, \\
\widetilde{\delta}^{(1)}=\delta^{(1)}-2 \rho^{(0)} \rho^{(1)}, & \widetilde{\rho}^{(1)}=\rho^{(1)} .
\end{array}
$$

$(\widetilde{H} \rightarrow \mathbb{C}, \widetilde{\nabla})$ is of the same type $($ Sem $)$ or $($ Bra $)$ or $($ Reg $)$ or $(N i l)$ as $(H \rightarrow \mathbb{C}, \nabla)$. The following table characterizes of which type the $(T E)$-structures $(H \rightarrow \mathbb{C}, \nabla)$ and $(\widetilde{H} \rightarrow \mathbb{C}, \widetilde{\nabla})$ are

\begin{tabular}{c|c|c|c}
\hline$($ Sem $)$ & $($ Bra $)$ & $($ Reg $)$ & $($ Log $)$ \\
\hline$\widetilde{\delta}^{(0)} \neq 0$ & $\widetilde{\delta}^{(0)}=0, \widetilde{\delta}^{(1)} \neq 0$ & $\widetilde{\delta}^{(0)}=\widetilde{\delta}^{(1)}=0, \widetilde{\mathcal{U}} \neq 0$ & $\widetilde{\mathcal{U}}=0$ \\
\hline
\end{tabular}

Especially, $\widetilde{\mathcal{U}}=0$ implies $\widetilde{\delta}^{(0)}=\widetilde{\delta}^{(1)}=0$.

\subsection{The case (Sem)}

A $(T E)$-structure over a point with a semisimple endomorphism $\mathcal{U}$ with pairwise different eigenvalues is formally isomorphic to a socalled elementary model, and its holomorphic isomorphism class is determined by its Stokes structure. These two facts are well known. A good reference is [21, Chapter II, Sections 5 and 6]. The older reference [16] considers only the underlying meromorphic bundle, so $\left(\mathcal{O}(H)_{0} \otimes_{\mathbb{C}\{z\}} \mathbb{C}\{z\}\left[z^{-1}\right], \nabla\right)$.

In order to formulate the result for rank $2(T E)$-structures more precisely, we need some notation.

Definition 4.4. Choose numbers $u_{1}, u_{2}, \alpha_{1}, \alpha_{2} \in \mathbb{C}$. Consider the flat bundle $H^{\prime} \rightarrow \mathbb{C}^{*}$ with flat connection $\nabla$ and a basis $\underline{f}=\left(f_{1}, f_{2}\right)$ of global flat multivalued sections $f_{1}$ and $f_{2}$ with the monodromy

$$
\underline{f}\left(z \cdot \mathrm{e}^{2 \pi \mathrm{i}}\right)=\underline{f}(z)\left(\begin{array}{cc}
\mathrm{e}^{-2 \pi \mathrm{i} \alpha_{1}} & 0 \\
0 & \mathrm{e}^{-2 \pi \mathrm{i} \alpha_{2}}
\end{array}\right) .
$$

The new basis $\underline{v}=\left(v_{1}, v_{2}\right)$ which is defined by

$$
\underline{v}(z)=\underline{f}(z)\left(\begin{array}{cc}
\mathrm{e}^{u_{1} / z} z^{\alpha_{1}} & 0 \\
0 & \mathrm{e}^{u_{2} / z} z^{\alpha_{2}}
\end{array}\right)
$$


(for some choice of $\log (z))$ is univalued. It defines a $(T E)$-structure with

$$
z^{2} \nabla_{\partial_{z} \underline{v}}=\underline{v} \cdot B \quad \text { and } \quad B=\left(\begin{array}{cc}
-u_{1}+z \alpha_{1} & 0 \\
0 & -u_{2}+z \alpha_{2}
\end{array}\right)
$$

This (TE)-structure is called an elementary model. The numbers $\alpha_{1}$ and $\alpha_{2}$ are called the regular singular exponents. The formal invariants $\delta^{(0)}, \rho^{(0)}, \delta^{(1)}, \rho^{(1)} \in \mathbb{C}$ of the $(T E)$-structure and the tuple $\left(u_{1}, u_{2}, \alpha_{1}, \alpha_{2}\right)$ (up to joint exchange of the indices 1 and 2 ) are equivalent because of

$$
\begin{aligned}
& \delta^{(0)}-\left(\rho^{(0)}\right)^{2}=-\frac{1}{4}\left(u_{1}-u_{2}\right)^{2}, \quad \rho^{(0)}=-\frac{u_{1}+u_{2}}{2}, \\
& \delta^{(1)}-2 \rho^{(0)} \rho^{(1)}=\frac{u_{1}-u_{2}}{2}\left(\alpha_{1}-\alpha_{2}\right), \quad \rho^{(1)}=\frac{\alpha_{1}+\alpha_{2}}{2} .
\end{aligned}
$$

Therefore also the tuple $\left(u_{1}, u_{2}, \alpha_{1}, \alpha_{2}\right)$ (up to joint exchange of the indices 1 and 2 ) is a formal invariant of the $(T E)$-structure.

\section{Theorem 4.5.}

(a) Any rank 2 (TE)-structure over a point with endomorphism $\mathcal{U}$ with two different eigenvalues is formally isomorphic to a unique elementary model in Definition 4.4. Here $-u_{1}$ and $-u_{2}$ are the eigenvalues of $\mathcal{U}$.

(b) The (TE)-structure in (a) is up to holomorphic isomorphism determined by the numbers $u_{1}, u_{2}, \alpha_{1}, \alpha_{2}$ and two more numbers $s_{1}, s_{2} \in \mathbb{C}$, the Stokes parameters. It is holomorphically isomorphic to the elementary model to which it is formally isomorphic if and only if $s_{1}=s_{2}=0$.

(c) Any such tuple $\left(u_{1}, u_{2}, \alpha_{1}, \alpha_{2}, s_{1}, s_{2}\right) \in\left(\mathbb{C}^{2} \backslash\left\{\left(u_{1}, u_{1}\right) \mid u_{1} \in \mathbb{C}\right\}\right) \times \mathbb{C}^{4}$ determines such $a(T E)$-structure. A second tuple $\left(\widetilde{u}_{1}, \widetilde{u}_{2}, \widetilde{\alpha}_{1}, \widetilde{\alpha}_{2}, \widetilde{s}_{1}, \widetilde{s}_{2}\right) \neq\left(u_{1}, u_{2}, \alpha_{1}, \alpha_{2}, s_{1}, s_{2}\right)$ determines an isomorphic (TE)-structure if and only if $\left(\widetilde{u}_{1}, \widetilde{u}_{2}, \widetilde{\alpha}_{1}, \widetilde{\alpha}_{2}, \widetilde{s}_{1}, \widetilde{s}_{2}\right)=\left(u_{2}, u_{1}, \alpha_{2}, \alpha_{1}, s_{2}, s_{1}\right)$.

Part (a) follows for example from [21, Chapter II, Theorem 5.7] together with [21, Chapter II, Remark 5.8] (Theorem 5.7 considers only the underlying meromorphic bundle; Remark 5.8 takes care of the holomorphic bundle). For the parts $(b)$ and $(c)$, one needs to deal in detail with the Stokes structure. We will not do it here, as the semisimple case is not central in this paper. We refer to [21, Chapter II, Sections 5 and 6] or to [14].

\section{Remarks 4.6.}

(i) Malgrange's unfolding result, Theorem 3.16(c), applies to these (TE)-structures. Such a $(T E)$-structure has a unique universal unfolding. The parameters $\left(\alpha_{1}, \alpha_{2}, s_{1}, s_{2}\right)$ are constant, the parameters $\left(u_{1}, u_{2}\right)$ are local coordinates on the base space. The base space is an $F$-manifold of type $A_{1}^{2}$ with Euler field $E=u_{1} e_{1}+u_{2} e_{2}$. See Remark 5.3(iii).

(ii) We do not offer normal forms for the $(T E)$-structures in Theorem 4.5 for three reasons: (1) As said in $(i)$, the $(T E)$-structures above unfold uniquely to $(T E)$-structures over germs of $F$-manifolds. In that sense they are easy to deal with. (2) It looks difficult to write down normal forms. (3) Normal forms should be considered together with the Stokes parameters, and the corresponding Riemann-Hilbert map from the space of monodromy data $\left(\alpha_{1}, \alpha_{2}, s_{1}, s_{2}\right)$ to a space of parameters for normal forms should be studied. This is a nontrivial project, which does not fit into the main aims of this paper. 


\subsection{Joint considerations on the cases (Bra), (Reg) and (Log)}

Notation 4.7. We shall use the following matrices,

$$
C_{1}:=\mathbf{1}_{2}, \quad C_{2}:=\left(\begin{array}{cc}
0 & 0 \\
1 & 0
\end{array}\right), \quad D:=\left(\begin{array}{cc}
1 & 0 \\
0 & -1
\end{array}\right), \quad E:=\left(\begin{array}{cc}
0 & 1 \\
0 & 0
\end{array}\right),
$$

and the relations between them,

$$
\begin{aligned}
& C_{2}^{2}=0, \quad D^{2}=C_{1}, \quad E^{2}=0, \\
& C_{2} D=C_{2}=-D C_{2}, \quad\left[C_{2}, D\right]=2 C_{2}, \\
& C_{2} E=\frac{1}{2}\left(C_{1}-D\right), \quad E C_{2}=\frac{1}{2}\left(C_{1}+D\right), \quad\left[C_{2}, E\right]=-D, \\
& D E=E=-E D, \quad[D, E]=2 E .
\end{aligned}
$$

Consider a $(T E)$-structure $(H \rightarrow \mathbb{C}, \nabla)$ over a point with $\mathcal{U}$ of type (Bra), (Reg) or (Log). Then $\mathcal{U}$ has only one eigenvalue, which is $\rho^{(0)} \in \mathbb{C}$. We can and will restrict to $\mathbb{C}\{z\}$-bases $\underline{v}$ of $\mathcal{O}(H)_{0}$ such that the matrix $B \in M_{2 \times 2}(\mathbb{C}\{z\})$ with $z^{2} \nabla_{\partial_{z}} \underline{v}=\underline{v} \cdot B$ has the shape

$$
B=b_{1} C_{1}+b_{2} C_{2}+z b_{3} D+z b_{4} E \quad \text { with } \quad b_{1}, b_{2}, b_{3}, b_{4} \in \mathbb{C}\{z\} .
$$

Write as in Remark $3.2 B=\sum_{k \geq 0} B^{(k)} z^{k}$ with $B^{(k)} \in M_{2 \times 2}(\mathbb{C})$, and write

$$
\begin{array}{ll}
b_{j}=\sum_{k \geq 0} b_{j}^{(k)} z^{k} & \text { with } \quad b_{j}^{(k)} \in \mathbb{C} \quad \text { for } \quad j \in\{1,2\}, \\
z b_{j}=\sum_{k \geq 1} b_{j}^{(k)} z^{k} & \text { with } \quad b_{j}^{(k)} \in \mathbb{C} \quad \text { for } \quad j \in\{3,4\} .
\end{array}
$$

Then the formal invariants $\delta^{(0)}, \rho^{(0)}, \delta^{(1)}$ and $\rho^{(1)}$ of Lemma 3.9 are given by

$$
\begin{aligned}
& \rho^{(0)}=b_{1}^{(0)}, \quad \rho^{(1)}=b_{1}^{(1)}, \\
& \delta^{(0)}-\left(\rho^{(0)}\right)^{2}=0, \quad \delta^{(1)}-2 \rho^{(0)} \rho^{(1)}=-b_{2}^{(0)} b_{4}^{(1)} .
\end{aligned}
$$

We are in the case (Bra) if $b_{2}^{(0)} \neq 0$ and $b_{4}^{(1)} \neq 0$, in the case (Reg) if $b_{2}^{(0)} \neq 0$ and $b_{4}^{(1)}=0$, and in the case $(\log )$ if $b_{2}^{(0)}=0$.

Consider $T \in \mathrm{GL}_{2}(\mathbb{C}\{z\})$ and the new basis $\underline{\widetilde{v}}=\underline{v} \cdot T$ and its matrix $\widetilde{B}=\sum_{k \geq 0} \widetilde{B}^{(k)} z^{k}$

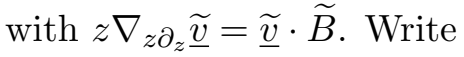

$$
T=\tau_{1} C_{1}+\tau_{2} C_{2}+\tau_{3} D+\tau_{4} E \quad \text { with } \quad \tau_{j}=\sum_{k \geq 0} \tau_{j}^{(k)} z^{k}, \quad \tau_{j}^{(k)} \in \mathbb{C} .
$$

Then $\widetilde{B}$ is determined by $(3.11)$, which is

$$
\begin{aligned}
0= & z^{2} \partial_{z} T+B \cdot T-T \cdot \widetilde{B} \\
= & C_{1}\left(z^{2} \partial_{z} \tau_{1}+\left(b_{1}-\widetilde{b}_{1}\right) \tau_{1}+z\left(b_{4}-\widetilde{b}_{4}\right) \frac{\tau_{2}}{2}+z\left(b_{3}-\widetilde{b}_{3}\right) \tau_{3}+\left(b_{2}-\widetilde{b}_{2}\right) \frac{\tau_{4}}{2}\right) \\
& +C_{2}\left(z^{2} \partial_{z} \tau_{2}+\left(b_{2}-\widetilde{b}_{2}\right) \tau_{1}+\left(b_{1}-\widetilde{b}_{1}\right) \tau_{2}+z\left(-b_{3}-\widetilde{b}_{3}\right) \tau_{2}+\left(b_{2}+\widetilde{b}_{2}\right) \tau_{3}\right) \\
& +D\left(z^{2} \partial_{z} \tau_{3}+z\left(b_{3}-\widetilde{b}_{3}\right) \tau_{1}+z\left(b_{4}+\widetilde{b}_{4}\right) \frac{\tau_{2}}{2}+\left(b_{1}-\widetilde{b}_{1}\right) \tau_{3}+\left(-b_{2}-\widetilde{b}_{2}\right) \frac{\tau_{4}}{2}\right) \\
& +E\left(z^{2} \partial_{z} \tau_{4}+z\left(b_{4}-\widetilde{b}_{4}\right) \tau_{1}+z\left(-b_{4}-\widetilde{b}_{4}\right) \tau_{3}+\left(b_{1}-\widetilde{b}_{1}\right) \tau_{4}+z\left(b_{3}+\widetilde{b}_{3}\right) \tau_{4}\right) .
\end{aligned}
$$

We will use this quite often in order to construct or compare normal forms. The following immediate corollary of the proof of Lemma 3.11 provides a reduction of $b_{1}$. 
Corollary 4.8. The base change matrix $T=\mathrm{e}^{g} \cdot C_{1}$ with $g$ as in (3.15) leads to $\widetilde{b}_{j}$ with

$$
\widetilde{b}_{1}=b_{1}^{(0)}+z b_{1}^{(1)}=\rho^{(0)}+z \rho^{(1)}, \quad \widetilde{b}_{2}=b_{2}, \quad \widetilde{b}_{3}=b_{3}, \quad \widetilde{b}_{4}=b_{4},
$$

From now on we will work in this section only with bases $\underline{v}$ with $b_{1}=\rho^{(0)}+z \rho^{(1)}$. This is justified by Corollary 4.8.

Furthermore, we will consider from now on in this section mainly $(T E)$-structures with trace free pole part (Definition 3.8, $\rho^{(0)}=\frac{1}{2} \operatorname{tr} \mathcal{U}=0$ ). See Lemmata 3.10 and 3.11 for the relation to the general case.

The next lemma separates the cases (Bra) and (Reg).

Lemma 4.9. Consider a (TE)-structure over a point with $\mathcal{U}$ of type (Bra) or type (Reg) and with trace free pole part (so $\mathcal{U}$ is nilpotent but not 0$)$.

The (TE)-structure is regular singular if and only if it is of type (Reg). If it is of type (Bra), then the pullback of $\mathcal{O}(H)_{0} \otimes_{\mathbb{C}\{z\}} \mathbb{C}\{z\}\left[z^{-1}\right]$ by the map $\mathbb{C} \rightarrow \mathbb{C}, x \mapsto x^{4}=z$, is the space of germs at 0 of sections of a meromorphic bundle on $\mathbb{C}$ with a meromorphic connection with an order 3 pole at 0 with semisimple pole part with eigenvalues $\kappa_{1}$ and $\kappa_{2}=-\kappa_{1}$ with $-\frac{1}{4} \kappa_{1}^{2}=\delta^{(1)} \in \mathbb{C}^{*}$. Thus $\kappa_{1}^{2}$ is a formal invariant of the $(T E)$-structure of type (Bra).

Proof. Consider a $\mathbb{C}\{z\}$-basis $\underline{v}$ of $\mathcal{O}(H)_{0}$ such that its matrix $B$ is as in (4.3) and such that $b_{1}=z \rho^{(1)}$. This is possible by Corollary 4.8 and the assumption $\rho^{(0)}=0$. As $\mathcal{U}$ is nilpotent, but not $0, b_{2}^{(0)} \neq 0$. Now $\delta^{(1)}=-b_{2}^{(0)} b_{4}^{(1)}$, so $\delta^{(1)} \neq 0 \Longleftrightarrow b_{4}^{(1)} \neq 0$.

Consider the case $b_{4}^{(1)} \neq 0$, and consider the pullback of the $(T E)$-structure by the map $\mathbb{C} \rightarrow \mathbb{C}, x \mapsto x^{4}=z$. Then $\frac{\mathrm{d} z}{z}=4 \frac{\mathrm{d} x}{x}$ and $z \partial_{z}=\frac{1}{4} x \partial_{x}$ and

$$
\begin{aligned}
\nabla_{x \partial_{x} \underline{v}}=\underline{v} \cdot 4 & \sum_{k \geq 0} B^{(k)} x^{4 k-4}, \\
\nabla_{x \partial_{x}}\left(\underline{v} \cdot x^{D}\right)= & \left(\underline{v} \cdot x^{D}\right) 4 \\
& \times\left(x^{-2} \sum_{k \geq 0}\left(b_{2}^{(k)} C_{2}+b_{4}^{(k+1)} E\right) x^{4 k}+\rho^{(1)} C_{1}+\left(\frac{1}{4}+\sum_{k \geq 0} b_{3}^{(k+1)} x^{4 k}\right) D\right) .
\end{aligned}
$$

One sees a pole of order 3 with matrix $4\left(b_{2}^{(0)} C_{2}+b_{4}^{(1)} E\right)$ of the pole part. It is tracefree and has the eigenvalues $\kappa_{1}$ and $\kappa_{2}=-\kappa_{1}$ with $\kappa_{1}^{2}=4 b_{2}^{(0)} b_{4}^{(1)} \in \mathbb{C}^{*}$. This shows the claims in the case $b_{4}^{(1)} \neq 0$.

Consider the case $b_{4}^{(1)}=0$, and consider the pullback of the $(T E)$-structure by the map $\mathbb{C} \rightarrow \mathbb{C}, x \mapsto x^{2}=z$. Then $\frac{\mathrm{d} z}{z}=2 \frac{\mathrm{d} x}{x}$ and $z \partial_{z}=\frac{1}{2} x \partial_{x}$ and

$$
\begin{aligned}
\nabla_{x \partial_{x} \underline{v}}=\underline{v} \cdot 2 \sum_{k \geq 0} & B^{(k)} x^{2 k-2}, \\
\nabla_{x \partial_{x}}\left(\underline{v} \cdot x^{D}\right)= & \left(\underline{v} \cdot x^{D}\right) 2 \\
& \times\left(\rho^{(1)} C_{1}+\frac{1}{2} D+\sum_{k \geq 0}\left(b_{2}^{(k)} C_{2}+b_{4}^{(k+2)} E+b_{3}^{(k+1)} D\right) x^{2 k}\right) .
\end{aligned}
$$

One sees a logarithmic pole. Therefore also the sections $v_{1}$ and $v_{2}$ have moderate growth, and the (TE)-structure is regular singular.

Remark 4.10. The two transformations in (4.7) (for the case (Bra)) and (4.8) (for the case (Reg)) are special cases of a systematic development of such ramified gauge transformations in [2] (a short description is given in [24, p. 17]). The basic idea goes back to the shearing transformations of Fabry (see [8] and [24, p. 4]). 


\subsection{The case (Bra)}

The following theorem gives complete control on the (TE)-structures over a point of the type (Bra). Here $\operatorname{Eig}\left(M^{\text {mon }}\right) \subset \mathbb{C}$ is the set of eigenvalues of the monodromy of such a $(T E)$-structure (it has 1 or 2 elements).

\section{Theorem 4.11.}

(a) Consider a (TE)-structure over a point of the type (Bra). The formal invariants $\rho^{(0)}, \rho^{(1)}$ and $\delta^{(1)} \in \mathbb{C}$ from Lemma 3.9 and the set $\operatorname{Eig}\left(M^{\text {mon }}\right)$ are invariants of its isomorphism class. Together they form a complete set of invariants. That means, the isomorphism class of the (TE)-structure is determined by these invariants.

(b) Any such $(T E)$-structure has a $\mathbb{C}\{z\}$-basis $\underline{v}$ of $\mathcal{O}(H)_{0}$ such that its matrix is in Birkhoff normal form, and more precisely, the matrix $B$ has the shape

$$
B=\left(\rho^{(0)}+z \rho^{(1)}\right) C_{1}+b_{2}^{(0)} C_{2}+z b_{3}^{(1)} D+z b_{4}^{(1)} E,
$$

where $b_{2}^{(0)}, b_{4}^{(1)} \in \mathbb{C}^{*}$ and $b_{3}^{(1)} \in \mathbb{C}$ satisfy $-b_{2}^{(0)} b_{4}^{(1)}=\delta^{(1)}-2 \rho^{(0)} \rho^{(1)}$ and $\operatorname{Eig}\left(M^{\text {mon }}\right)=$ $\left\{\mathrm{e}^{-2 \pi \mathrm{i}\left(\rho^{(1)} \pm b_{3}^{(1)}\right)}\right\}$.

\section{Remarks 4.12.}

(i) Because of part $(a)$, two Birkhoff normal forms as in $(4.9)$ with data $\left(\rho^{(0)}, \rho^{(1)}, b_{2}^{(0)}\right.$, $\left.b_{3}^{(1)}, b_{4}^{(1)}\right)$ and $\left(\widetilde{\rho}^{(0)}, \widetilde{\rho}^{(1)}, \widetilde{b}_{2}^{(0)}, \widetilde{b}_{3}^{(1)}, \widetilde{b}_{4}^{(1)}\right)$ give isomorphic $(T E)$-structures if and only if $\widetilde{\rho}^{(0)}=$ $\rho^{(0)}, \widetilde{\rho}^{(1)}=\rho^{(1)}, \widetilde{b}_{2}^{(0)} \widetilde{b}_{4}^{(1)}=b_{2}^{(0)} b_{4}^{(1)}$ and $\widetilde{b}_{3}^{(1)} \in\left\{ \pm b_{3}^{(1)}+k \mid k \in \mathbb{Z}\right\}$. However, the pure (TLE)structures which they define, are isomorphic only if additionally $\widetilde{b}_{3}^{(1)} \in\left\{ \pm b_{3}^{(1)}\right\}$.

(ii) We could restrict to Birkhoff normal forms with $b_{2}^{(0)}=1$ or with $b_{4}^{(1)}=1$. Though in view of the (TE)-structures in the 4 th case in Theorem 6.3 we prefer not to do that.

Proof of Theorem 4.11. The proof has 3 steps.

Step 1: Birkhoff normal forms exist. In order to show this, it is sufficient to prove the hypothesis in Theorem 3.20(b). The hypothesis says that the germ of the meromorphic bundle underlying a $(T E)$-structure of type (Bra) is irreducible. A proof by calculation is given in the proof of Lemma 28 in [6]. Though this is also well known as the rank is two and the slope is $\frac{1}{2}$ (see Remark 4.2).

Step 2: Analysis of the Birkhoff normal forms. The matrix $B$ of a Birkhoff normal form can be chosen with $b_{1}=\rho^{(0)}+z \rho^{(1)}$ because of Corollary 4.8. Then it has the shape

$$
B=\left(\rho^{(0)}+z \rho^{(1)}\right) C_{1}+\left(b_{2}^{(0)}+z b_{2}^{(1)}\right) C_{2}+z b_{3}^{(1)} D+z b_{4}^{(1)} E
$$

with $b_{2}^{(0)} \neq 0$ and $b_{4}^{(1)} \neq 0$.

Consider the new basis $\underline{\widetilde{v}}=\underline{v} \cdot T$ and its matrix $\widetilde{B}$, where

$$
T=C_{1}+\tau_{2}^{(0)} C_{2} \quad \text { for some } \tau_{2}^{(0)} \in \mathbb{C} .
$$

Equation (4.6) gives

$$
\begin{array}{ll}
0=\left(b_{1}-\widetilde{b}_{1}\right)+z\left(b_{4}^{(1)}-\widetilde{b}_{4}\right) \frac{\tau_{2}^{(0)}}{2}, & 0=\left(b_{2}-\widetilde{b}_{2}\right)+\left(b_{1}-\widetilde{b}_{1}\right) \tau_{2}^{(0)}+z\left(-b_{3}^{(1)}-\widetilde{b}_{3}\right) \tau_{2}^{(0)}, \\
0=\left(b_{3}^{(1)}-\widetilde{b}_{3}\right)+\left(b_{4}^{(1)}+\widetilde{b}_{4}\right) \frac{\tau_{2}^{(0)}}{2}, & 0=\left(b_{4}^{(1)}-\widetilde{b}_{4}\right),
\end{array}
$$


so

$$
\begin{aligned}
& \widetilde{b}_{4}=\widetilde{b}_{4}^{(1)}=b_{4}^{(1)}, \quad \widetilde{b}_{1}=b_{1}, \quad \widetilde{b}_{3}=\widetilde{b}_{3}^{(1)}=b_{3}^{(1)}+b_{4}^{(1)} \tau_{2}^{(0)}, \\
& \widetilde{b}_{2}^{(0)}=b_{2}^{(0)}, \quad \widetilde{b}_{2}^{(1)}=b_{2}^{(1)}-2 b_{3}^{(1)} \tau_{2}^{(0)}-b_{4}^{(1)}\left(\tau_{2}^{(0)}\right)^{2} .
\end{aligned}
$$

$\tau_{2}^{(0)}$ can be chosen such that $\widetilde{b}_{2}^{(1)}=0$. Then the Birkhoff normal form $\widetilde{B}$ has the shape in (4.9).

Suppose now that $B$ has this shape, so $b_{2}=b_{2}^{(0)}$. The choice $\tau_{2}^{(0)}:=-2 b_{3}^{(1)} / b_{4}^{(1)}$ in $(4.10)$ leads to

$$
\widetilde{b}_{1}=b_{1}, \quad \widetilde{b}_{2}=b_{2}, \quad \widetilde{b}_{4}=b_{4} \quad \text { and } \quad \widetilde{b}_{3}=-b_{3} .
$$

Consider the new basis $\underline{\widetilde{v}}=\underline{v} \cdot T$ and its matrix $\widetilde{B}$, where

$$
T=C_{1}+\tau_{3}^{(0)} D \quad \text { for some } \quad \tau_{3}^{(0)} \in \mathbb{C} \backslash\{ \pm 1\} .
$$

Equation (4.6) gives

$$
\begin{aligned}
& 0=\left(b_{1}-\widetilde{b}_{1}\right)+z\left(b_{3}^{(1)}-\widetilde{b}_{3}\right) \tau_{3}^{(0)}, \\
& 0=\left(b_{2}^{(0)}-\widetilde{b}_{2}\right)+\left(b_{2}^{(0)}+\widetilde{b}_{2}\right) \tau_{3}^{(0)}, \\
& 0=z\left(b_{3}^{(1)}-\widetilde{b}_{3}\right)+\left(b_{1}-\widetilde{b}_{1}\right) \tau_{3}^{(0)}, \\
& 0=\left(b_{4}^{(1)}-\widetilde{b}_{4}\right)+\left(-b_{4}^{(1)}-\widetilde{b}_{4}\right) \tau_{3}^{(0)},
\end{aligned}
$$

SO

$$
\widetilde{b}_{1}=b_{1}, \quad \widetilde{b}_{3}=b_{3}^{(1)}, \quad \widetilde{b}_{2}=b_{2}^{(0)} \frac{1+\tau_{3}^{(0)}}{1-\tau_{3}^{(0)}}, \quad \widetilde{b}_{4}=b_{4}^{(1)} \frac{1-\tau_{3}^{(0)}}{1+\tau_{3}^{(0)}} .
$$

So, in a Birkhoff normal form in (4.9), one can change $b_{2}^{(0)}$ and $b_{4}^{(1)}$ arbitrarily with constant product $b_{2}^{(0)} b_{4}^{(1)}$ and without changing $b_{1}=\rho^{(0)}+z \rho^{(1)}$ and $b_{3}^{(1)}$.

Consider the new basis $\underline{\widetilde{v}}=\underline{v} \cdot T$ and its matrix $\widetilde{B}$, where

$$
T=\left(1+z \tau_{1}^{(1)}\right) C_{1}+\tau_{2}^{(0)} C_{2}+z \tau_{3}^{(1)} D+z \tau_{4}^{(1)} E, \quad \text { for some } \quad \tau_{1}^{(1)}, \tau_{2}^{(0)}, \tau_{3}^{(1)}, \tau_{4}^{(1)} \in \mathbb{C} .
$$

We are searching for coefficients $\tau_{1}^{(1)}, \tau_{2}^{(0)}, \tau_{3}^{(1)}, \tau_{4}^{(1)} \in \mathbb{C}$ such that

$$
\widetilde{b}_{1}=b_{1}, \quad \widetilde{b}_{2}=b_{2}, \quad \widetilde{b}_{4}=b_{4}, \quad \widetilde{b}_{3}=b_{3}+\varepsilon \quad \text { with } \quad \varepsilon= \pm 1 .
$$

Under these constraints, (4.6) gives

$$
\begin{aligned}
& 0=\tau_{1}^{(1)}-\varepsilon \tau_{3}^{(1)}, \\
& 0=\left(-2 b_{3}^{(1)}-\varepsilon\right) \tau_{2}^{(0)}+2 b_{2}^{(0)} \tau_{3}^{(1)}, \\
& 0=z \tau_{3}^{(1)}-\varepsilon\left(1+z \tau_{1}^{(1)}\right)+b_{4}^{(1)} \tau_{2}^{(0)}-b_{2}^{(0)} \tau_{4}^{(1)}, \\
& 0=\tau_{4}^{(1)}-2 b_{4}^{(1)} \tau_{3}^{(1)}+\left(2 b_{3}^{(1)}+\varepsilon\right) \tau_{4}^{(1)} .
\end{aligned}
$$

With $\tau_{1}^{(1)}=\varepsilon \tau_{3}^{(1)}$, these equations boil down to the inhomogeneous linear system of equations

$$
\left(\begin{array}{l}
0 \\
\varepsilon \\
0
\end{array}\right)=\left(\begin{array}{ccc}
-2 b_{3}^{(1)}-\varepsilon & 2 b_{2}^{(0)} & 0 \\
b_{4}^{(1)} & 0 & -b_{2}^{(0)} \\
0 & -2 b_{4}^{(1)} & 2 b_{3}^{(1)}+\varepsilon+1
\end{array}\right)\left(\begin{array}{c}
\tau_{2}^{(0)} \\
\tau_{3}^{(1)} \\
\tau_{4}^{(1)}
\end{array}\right) .
$$


The determinant of the $3 \times 3$ matrix is $-2 b_{2}^{(0)} b_{4}^{(1)} \neq 0$. Therefore the system (4.15) has a unique solution $\left(\tau_{2}^{(0)}, \tau_{3}^{(1)}, \tau_{4}^{(1)}\right)^{t}$. Thus a new basis $\underline{\widetilde{v}}=\underline{v} \cdot T$ with (4.14) exists.

Iterating this construction, one finds that one can change the matrix $B$ in (4.9) by a holomorphic base change to a matrix $\widetilde{B}$ with

$$
\widetilde{b}_{1}=b_{1}, \quad \widetilde{b}_{2}=b_{2}, \quad \widetilde{b}_{4}=b_{4}, \quad \widetilde{b}_{3}=b_{3}+k,
$$

for any $k \in \mathbb{Z}$.

Putting together (4.11), (4.12), (4.13) and (4.16), one sees that two Birkhoff normal forms as in (4.9) with data $\left(\rho^{(0)}, \rho^{(1)}, b_{2}^{(0)}, b_{3}^{(1)}, b_{4}^{(1)}\right)$ and $\left(\widetilde{\rho}^{(0)}, \widetilde{\rho}^{(1)}, \widetilde{b}_{2}^{(0)}, \widetilde{b}_{3}^{(1)}, \widetilde{b}_{4}^{(1)}\right)$ give isomorphic $(T E)$ structures if $\widetilde{\rho}^{(0)}=\rho^{(0)}, \widetilde{\rho}^{(1)}=\rho^{(1)}, \widetilde{b}_{2}^{(0)} \widetilde{b}_{4}^{(1)}=b_{2}^{(0)} b_{4}^{(1)}$ and $\widetilde{b}_{3}^{(1)} \in\left\{ \pm b_{3}^{(1)}+k \mid k \in \mathbb{Z}\right\}$. This shows if in Remark 4.12(i).

Step 3: Discussion of the invariants. By Lemma 3.9, $\rho^{(0)}, \rho^{(1)}$ and $\delta^{(1)}$ are even formal invariants of the $(T E)$-structure. The set $\operatorname{Eig}\left(M^{\mathrm{mon}}\right)$ is obviously an invariant of the isomorphism class of the $(T E)$-structure.

The Birkhoff normal form in (4.9) gives a pure $(T L E)$-structure with a logarithmic pole at $\infty$. From its pole part at $\infty$ and Theorem 3.23(c) one reads off

$$
\operatorname{Eig}\left(M^{\text {mon }}\right)=\left\{\mathrm{e}^{-2 \pi \mathrm{i}\left(\rho^{(1)} \pm b_{3}^{(1)}\right)}\right\} .
$$

As $\rho^{(1)}$ is an invariant of the $(T E)$-structure, also the set $\left\{ \pm b_{3}^{(1)}+k \mid k \in \mathbb{Z}\right\}$ is an invariant of the $(T E)$-structure.

Together with Step 2, this shows only if in Remark $4.12(i)$ and all statements in Theorem 4.11.

Corollary 4.13. The monodromy of a (TE)-structure over a point of the type (Bra) has a $2 \times 2$ Jordan block if its eigenvalues coincide (equivalently, if $b_{3}^{(1)} \in \frac{1}{2} \mathbb{Z}$ for some (or any) Birkhoff normal form in Theorem 4.11(b)).

Proof. Consider a $(T E)$-structure over a point of the type (Bra) such that the eigenvalues of its monodromy coincide. Then for any Birkhoff normal form in Theorem $4.11(b) b_{3}^{(1)} \in \frac{1}{2} \mathbb{Z}$, and one can choose a Birkhoff normal form with $b_{3}^{(1)} \in\left\{0,-\frac{1}{2}\right\}$. The induced pure (TLE)-structure has at $\infty$ a logarithmic pole, and its residue endomorphism $\left[\nabla_{\widetilde{z} \partial_{\tilde{z}}}\right]$, where $\widetilde{z}=z^{-1}$, is given by the matrix $-\left(\rho^{(1)} C_{1}+b_{3}^{(1)} D+b_{4}^{(1)} E\right)$.

In the case $b_{3}^{(1)}=0$, the nonresonance condition in Theorem 3.23(c) is satisfied, so Theorem $3.23(c)$ can be applied. Because of $b_{4}^{(1)} \neq 0$, the monodromy has a $2 \times 2$ Jordan block.

In the case $b_{3}^{(1)}=-\frac{1}{2}$, the meromorphic base change

$$
\underline{\widetilde{v}}:=\underline{v} \cdot\left(\begin{array}{cc}
z & 0 \\
0 & 1
\end{array}\right)
$$

gives the new connection matrix

$$
\widetilde{B}=\left(\rho^{(0)}+z\left(\rho^{(1)}+\frac{1}{2}\right)\right) C_{1}+z b_{2}^{(0)} C_{2}+b_{4}^{(1)} E .
$$

Again, the pole at $\infty$ is logarithmic. Now the nonresonance condition in Theorem 3.23(c) is satisfied. Because of $b_{2}^{(0)} \neq 0$, the monodromy has a $2 \times 2$ Jordan block.

For $(T E)$-structures of the type (Bra), formal isomorphism is coarser than holomorphic isomorphism. 
Lemma 4.14. Consider a (TE)-structure over a point of the type (Bra). By Lemma 3.9, the numbers $\rho^{(0)}, \rho^{(1)}$ and $\delta^{(1)}$ are formal invariants of the $(T E)$-structure.

(a) The set $\operatorname{Eig}\left(M^{\text {mon }}\right)$ and the equivalent set $\left\{ \pm b_{3}^{(1)}+k \mid k \in \mathbb{Z}\right\}$ are holomorphic invariants, but not formal invariants.

(b) The (TE)-structure with Birkhoff normal form in (4.9) is formally isomorphic to the (TE)structure with Birkhoff normal form in (4.9) with the same values $\rho^{(0)}, \rho^{(1)}, b_{2}^{(0)}$ and $b_{4}^{(1)}$, but with an arbitrary $\widetilde{b}_{3}^{(1)}$.

Proof. Part (a) follows from part (b). For the proof of part (b), we have to find $T \in \mathrm{GL}_{2}(\mathbb{C}[[z]])$ such that $T, B$ in (4.9) and

$$
\widetilde{B}=\left(\rho^{(0)}+z \rho^{(1)}\right) C_{1}+b_{2}^{(0)} C_{2}+z \widetilde{b}_{3}^{(1)} D+z b_{4}^{(1)} E
$$

with $\widetilde{b}_{3}^{(1)} \in \mathbb{C}$ arbitrary satisfy (4.6). Here (4.6) says

$$
\begin{aligned}
& 0=z \partial_{z} \tau_{1}+\left(b_{3}^{(1)}-\widetilde{b}_{3}^{(1)}\right) \tau_{3}, \\
& 0=z^{2} \partial_{z} \tau_{2}+z\left(-b_{3}^{(1)}-\widetilde{b}_{3}^{(1)}\right) \tau_{2}+2 b_{2}^{(0)} \tau_{3}, \\
& 0=z^{2} \partial_{z} \tau_{3}+z\left(b_{3}^{(1)}-\widetilde{b}_{3}^{(1)}\right) \tau_{1}+z b_{4}^{(1)} \tau_{2}-b_{2}^{(0)} \tau_{4}, \\
& 0=z \partial_{z} \tau_{4}-2 b_{4}^{(1)} \tau_{3}+\left(b_{3}^{(1)}+\widetilde{b}_{3}^{(1)}\right) \tau_{4} .
\end{aligned}
$$

This is equivalent to

$$
\begin{aligned}
& 0=\tau_{3}^{(0)}=\tau_{4}^{(0)} \\
& 0=k \tau_{1}^{(k)}+\left(b_{3}^{(1)}-\widetilde{b}_{3}^{(1)}\right) \tau_{3}^{(k)} \quad \text { for } \quad k \geq 1, \\
& 0=\left(k-1-b_{3}^{(1)}-\widetilde{b}_{3}^{(1)}\right) \tau_{2}^{(k-1)}+2 b_{2}^{(0)} \tau_{3}^{(k)} \quad \text { for } \quad k \geq 1, \\
& 0=(k-1) \tau_{3}^{(k-1)}+\left(b_{3}^{(1)}-\widetilde{b}_{3}^{(1)}\right) \tau_{1}^{(k-1)}+b_{4}^{(1)} \tau_{2}^{(k-1)}-b_{2}^{(0)} \tau_{4}^{(k)} \quad \text { for } \quad k \geq 1, \\
& 0=-2 b_{4}^{(1)} \tau_{3}^{(k)}+\left(k+b_{3}^{(1)}+\widetilde{b}_{3}^{(1)}\right) \tau_{4}^{(k)} \quad \text { for } \quad k \geq 1 .
\end{aligned}
$$

This is equivalent to

$$
\begin{aligned}
& \tau_{3}^{(0)}=\tau_{4}^{(0)}=0, \\
& \tau_{1}^{(k)}=\frac{-1}{k}\left(b_{3}^{(1)}-\widetilde{b}_{3}^{(1)}\right) \tau_{3}^{(k)} \quad \text { for } \quad k \geq 1, \\
& 2 b_{2}^{(0)} \tau_{3}^{(k)}=\left(b_{3}^{(1)}+\widetilde{b}_{3}^{(1)}+1-k\right) \tau_{2}^{(k-1)} \quad \text { for } \quad k \geq 1, \\
& b_{2}^{(0)} \tau_{4}^{(1)}=b_{4}^{(1)} \tau_{2}^{(0)}+\left(b_{3}^{(1)}-\widetilde{b}_{3}^{(1)}\right) \tau_{1}^{(0)}, \\
& b_{2}^{(0)} \tau_{4}^{(k)}=b_{4}^{(1)} \tau_{2}^{(k-1)}+\left(k-1+\frac{-1}{k-1}\left(b_{3}^{(1)}-\widetilde{b}_{3}^{(1)}\right)^{2}\right)\left(2 b_{2}^{(0)}\right)^{-1}\left(b_{3}^{(1)}+\widetilde{b}_{3}^{(1)}+2-k\right) \tau_{2}^{(k-2)} \\
& \quad \text { for } \quad k \geq 2, \\
& 0=b_{4}^{(1)} \tau_{2}^{(0)}+\left(1+b_{3}^{(1)}+\widetilde{b}_{3}^{(1)}\right)\left(b_{3}^{(1)}-\widetilde{b}_{3}^{(1)}\right) \tau_{1}^{(0)}, \\
& 0=b_{4}^{(1)}(2 k+1) \tau_{2}^{(k)}+\left(k+1+b_{3}^{(1)}+\widetilde{b}_{3}^{(1)}\right)\left(k+\frac{-1}{k}\left(b_{3}^{(1)}-\widetilde{b}_{3}^{(1)}\right)^{2}\right)\left(2 b_{2}^{(0)}\right)^{-1} \\
& \quad \times\left(b_{3}^{(1)}+\widetilde{b}_{3}^{(1)}+1-k\right) \tau_{2}^{(k-1)} \quad \text { for } \quad k \geq 1 .
\end{aligned}
$$

One can choose $\tau_{1}^{(0)} \in \mathbb{C}^{*}$ freely. Then the equations (4.17) have unique solutions $\tau_{1}-\tau_{1}^{(0)}, \tau_{2}$, $\tau_{3}, \tau_{4} \in \mathbb{C}[[z]]$. Therefore $T \in \mathrm{GL}_{2}(\mathbb{C}[[z]])$ exists such that $T, B$ as in (4.9) and $\widetilde{B}$ as above satisfy (4.6). This shows part (b). 


\section{Remarks 4.15.}

(i) Because of Lemma 4.14, the set $\operatorname{Eig}\left(M^{\text {mon }}\right)$ takes here the role of the Stokes structure: It distinguishes the holomorphic isomorphism classes within one formal isomorphism class.

(ii) The following (TE)-structures have trivial Stokes structure. The proof of Lemma 4.9 leads to these $(T E)$ structures. They are the $(T E)$-structures with $\operatorname{Eig}\left(M^{\text {mon }}\right)=\left\{\lambda_{1}, \lambda_{2}\right\}$ with $\lambda_{2}=-\lambda_{1}$, respectively with $b_{3}^{(1)} \in\left( \pm \frac{1}{4}+\mathbb{Z}\right)$ for any Birkhoff normal form in (4.9).

Choose a number $\alpha^{(1)} \in \mathbb{C}$. Consider the rank 2 bundle $H^{\prime} \rightarrow \mathbb{C}^{*}$ with flat connection $\nabla$ and flat multivalued basis $\underline{f}=\left(f_{1}, f_{2}\right)$ with monodromy given by

$$
\underline{f}\left(z \mathrm{e}^{2 \pi \mathrm{i}}\right)=\underline{f}(z) \cdot \mathrm{ie}^{-2 \pi \mathrm{i} \alpha^{(1)}} \cdot\left(C_{2}+E\right) .
$$

The eigenvalues are $\pm \mathrm{ie}^{-2 \pi \mathrm{i} \alpha^{(1)}}$. Choose numbers $t_{1} \in \mathbb{C}$ and $t_{2} \in \mathbb{C}^{*}$. The following basis of $H^{\prime}$ is univalued.

$$
\underline{v}:=\underline{f} \cdot \mathrm{e}^{t_{1} / z} z^{\alpha^{(1)}}\left(\begin{array}{cc}
z^{-1 / 4} \mathrm{e}^{t_{2} z^{-1 / 2}} & z^{1 / 4} \mathrm{e}^{t_{2} z^{-1 / 2}} \\
z^{-1 / 4} \mathrm{e}^{-t_{2} z^{-1 / 2}} & -z^{1 / 4} \mathrm{e}^{-t_{2} z^{-1 / 2}}
\end{array}\right) .
$$

The matrix $B$ with $z^{2} \nabla_{\partial_{z}} \underline{v}=\underline{v} \cdot B$ is

$$
B=\left(-t_{1}+z \alpha^{(1)}\right) C_{1}-\frac{t_{2}}{2} C_{2}-z \frac{1}{4} D-z \frac{t_{2}}{2} E .
$$

So here $\rho^{(1)}=\alpha^{(1)}, \rho^{(0)}=-t_{1}, \delta^{(1)}-2 \rho^{(0)} \rho^{(1)}=-\frac{1}{4} t_{2}^{2}$.

(iii) Part (ii) generalizes to a $(T E)$-structure over $M=\mathbb{C}^{2}$ with coordinates $t=\left(t_{1}, t_{2}\right)$. Consider the rank 2 bundle $H^{\prime} \rightarrow \mathbb{C}^{*} \times M$ with flat connection and flat multivalued basis $\underline{f}=\left(f_{1}, f_{2}\right)$ with monodromy given by

$$
\underline{f}\left(z \mathrm{e}^{2 \pi \mathrm{i}}, t\right)=\underline{f}(z, t) \cdot \mathrm{ie}^{-2 \pi \mathrm{i} \alpha(1)} \cdot\left(C_{2}+E\right) .
$$

The basis $\underline{v}$ in (4.18) is univalued. The matrices $A_{1}, A_{2}$ and $B$ in its connection 1 -form $\Omega$ as in (3.2)-(3.4) are given by (4.19) and

$$
A_{1}=C_{1}, \quad A_{2}=C_{2}+z E .
$$

The restriction to a point $t \in \mathbb{C} \times \mathbb{C}^{*}$ is a $(T E)$-structure of type (Bra) with trivial Stokes structure. The restriction to a point $t \in \mathbb{C} \times\{0\}$ is a $(T E)$-structure of type (Log).

\subsection{The case (Reg) with $\operatorname{tr} \mathcal{U}=0$}

The (TE)-structures over a point of the type (Reg) with $\operatorname{tr} \mathcal{U}=0$ are the regular singular $(T E)$ structures over a point which are not logarithmic. They can be easily classified using elementary sections. Theorem 4.17 splits them into three cases (one in part $(a)$, two in part $(b): \alpha_{1}=\alpha_{2}$ and $\left.\alpha_{1}-\alpha_{2} \in \mathbb{N}\right)$.

Notation 4.16. Start with a $(T E)$-structure $(H \rightarrow \mathbb{C}, \nabla)$ of rank 2 over a point. Recall the notions from Definition 3.21: $H^{\prime}:=\left.H\right|_{\mathbb{C}^{*}}, M^{\text {mon }}, M_{s}^{\text {mon }}, M_{u}^{\text {mon }}, N^{\text {mon }}, \operatorname{Eig}\left(M^{\text {mon }}\right), H^{\infty}$, $H_{\lambda}^{\infty}, C^{\alpha}$ for $\alpha \in \mathbb{C}$ with $\mathrm{e}^{-2 \pi \mathrm{i} \alpha} \in \operatorname{Eig}\left(M^{\mathrm{mon}}\right), s(A, \alpha) \in C^{\alpha}$ for $A \in H_{\mathrm{e}^{-2 \pi \mathrm{i} \alpha}}^{\infty}, \operatorname{es}(\sigma, \alpha) \in C^{\alpha}$ for $\sigma$ a holomorphic section on $\left.H\right|_{U_{1} \backslash\{0\}}$ for $U_{1} \subset \mathbb{C}$ a neighborhood of 0 . Now the eigenvalues of $M^{\text {mon }}$ 
are called $\lambda_{1}$ and $\lambda_{2}\left(\lambda_{1}=\lambda_{2}\right.$ is allowed). The sheaf $\mathcal{V}>-\infty$ simplifies here to a $\mathbb{C}\{z\}\left[z^{-1}\right]$-vector space of dimension 2 ,

$$
V^{>-\infty}:=\left\{\begin{array}{lll}
\mathbb{C}\{z\}\left[z^{-1}\right] \cdot C^{\alpha_{1}} \oplus \mathbb{C}\{z\}\left[z^{-1}\right] \cdot C^{\alpha_{2}} & \text { if } \quad \lambda_{1} \neq \lambda_{2}, \\
\mathbb{C}\{z\}\left[z^{-1}\right] \cdot C^{\alpha_{1}} & \text { if } \lambda_{1}=\lambda_{2},
\end{array}\right.
$$

where $\alpha_{1}, \alpha_{2} \in \mathbb{C}$ with $\mathrm{e}^{-2 \pi \mathrm{i} \alpha_{j}}=\lambda_{j} . V^{>-\infty}$ is the space of sections of moderate growth.

Theorem 4.17. Consider a regular singular, but not logarithmic, rank 2 (TE)-structure $(H \rightarrow$ $\mathbb{C}, \nabla)$ over a point. Associate to it the data in the Notation 4.16.

(a) The case $N^{\mathrm{mon}}=0$ : There exist unique numbers $\alpha_{1}, \alpha_{2}$ with $\mathrm{e}^{-2 \pi \mathrm{i} \alpha_{j}}=\lambda_{j}$ and $\alpha_{1} \neq \alpha_{2}$ and the following properties: There exist elementary sections $s_{1} \in C^{\alpha_{1}} \backslash\{0\}$ and $s_{2} \in C^{\alpha_{2}} \backslash\{0\}$ and a number $t_{2} \in \mathbb{C}^{*}$ such that

$$
\begin{aligned}
\mathcal{O}(H)_{0} & =\mathbb{C}\{z\}\left(s_{1}+t_{2} s_{2}\right) \oplus \mathbb{C}\{z\}\left(z s_{2}\right) \\
& =\mathbb{C}\{z\}\left(s_{2}+t_{2}^{-1} s_{1}\right) \oplus \mathbb{C}\{z\}\left(z s_{1}\right) .
\end{aligned}
$$

The isomorphism class of the (TE)-structure is uniquely determined by the information $N^{\text {mon }}=0$ and the set $\left\{\alpha_{1}, \alpha_{2}\right\}$. The numbers $\alpha_{1}$ and $\alpha_{2}$ are called leading exponents.

(b) The case $N^{\text {mon }} \neq 0$ (thus $\lambda_{1}=\lambda_{2}$ ): There exist unique numbers $\alpha_{1}, \alpha_{2}$ with $\mathrm{e}^{-2 \pi \mathrm{i} \alpha_{j}}=\lambda_{1}$ and $\alpha_{1}-\alpha_{2} \in \mathbb{N}_{0}$ and the following properties: Choose any elementary section $s_{1} \in$ $C^{\alpha_{1}} \backslash \operatorname{ker}\left(z \nabla_{\partial_{z}}-\alpha_{1}: C^{\alpha_{1}} \rightarrow C^{\alpha_{1}}\right)$. The elementary section $s_{2} \in C^{\alpha_{2}}$ with

$$
\left(z \nabla_{\partial_{z}}-\alpha_{1}\right)\left(s_{1}\right)=z^{\alpha_{1}-\alpha_{2}} s_{2} .
$$

is a generator of $\operatorname{ker}\left(z \nabla_{\partial_{z}}-\alpha_{2}: C^{\alpha_{2}} \rightarrow C^{\alpha_{2}}\right)$. Then

$$
\mathcal{O}(H)_{0}=\mathbb{C}\{z\}\left(s_{1}+t_{2} s_{2}\right) \oplus \mathbb{C}\{z\}\left(z s_{2}\right)
$$

for some $t_{2} \in \mathbb{C}$. If $\alpha_{1}>\alpha_{2}$ then $t_{2}$ is in $\mathbb{C}^{*}$ and is independent of the choice of $s_{1}$. If $\alpha_{1}=\alpha_{2}$, then one can replace $s_{1}$ by $s_{1}^{[\text {new] }}:=s_{1}+t_{2} s_{2}$, and then $t_{2}^{[\text {new }]}=0$. The isomorphism class of the (TE)-structure is uniquely determined by the information $N^{\mathrm{mon}} \neq 0$ and the pair $\left(\alpha_{1}, \alpha_{2}\right)$ if $\alpha_{1}=\alpha_{2}$ and the triple $\left(\alpha_{1}, \alpha_{2}, t_{2}\right)$ if $\alpha_{1}>\alpha_{2}$. The numbers $\alpha_{1}$ and $\alpha_{2}$ are called leading exponents.

Proof. First, $(a)$ and $(b)$ are considered together. Let $\beta_{1}, \beta_{2} \in \mathbb{C}$ be the unique numbers with $\mathrm{e}^{-2 \pi \mathrm{i} \beta_{j}}=\lambda_{j}$ and $-1<\operatorname{Re}\left(\beta_{j}\right) \leq 0$. Choose elementary sections $\widetilde{s}_{1} \in C^{\beta_{1}}$ and $\widetilde{s}_{2} \in C^{\beta_{2}}$ which form a global basis of $H^{\prime}$. In the case $N^{\text {mon }} \neq 0$ (then $\beta_{1}=\beta_{2}$ ) choose them such that $\widetilde{s}_{1} \notin \operatorname{ker}\left(z \nabla_{\partial_{z}}-\beta_{1}: C^{\beta_{1}} \rightarrow C^{\beta_{1}}\right)$ and $\widetilde{s}_{2} \in \operatorname{ker}\left(z \nabla_{\partial_{z}}-\beta_{2}: C^{\beta_{2}} \rightarrow C^{\beta_{2}}\right)$.

Let $\sigma_{1}^{[1]}, \sigma_{2}^{[1]} \in \mathcal{O}(H)_{0}$ be a $\mathbb{C}\{z\}$-basis of $\mathcal{O}(H)_{0}$. Write

$$
\left(\sigma_{1}^{[1]}, \sigma_{2}^{[1]}\right)=\left(\widetilde{s}_{1}, \widetilde{s}_{2}\right)\left(\begin{array}{ll}
b_{11} & b_{12} \\
b_{21} & b_{22}
\end{array}\right) \quad \text { with } \quad b_{i j} \in \mathbb{C}\{z\}\left[z^{-1}\right] .
$$

Recall that the degree $\operatorname{deg}_{z} g$ of a Laurent series $g=\sum_{j \in \mathbb{Z}} g^{(j)} z^{j} \in \mathbb{C}\{z\}\left[z^{-1}\right]$ is the minimal $j$ with $g^{(j)} \neq 0$ if $g \neq 0$, and $\operatorname{deg}_{z} 0:=+\infty$.

In the case $N^{\text {mon }}=0$ and $\lambda_{1}=\lambda_{2}$ (then $\left.\beta_{1}=\beta_{2}\right)$, we suppose $\min \left(\operatorname{deg}_{z} b_{11}, \operatorname{deg}_{z} b_{12}\right) \leq$ $\min \left(\operatorname{deg}_{z} b_{21}, \operatorname{deg}_{z} b_{22}\right)$. If it does not hold a priori, we can exchange $\widetilde{s}_{1}$ and $\widetilde{s}_{2}$.

In any case, we suppose $\operatorname{deg}_{z} b_{11} \leq \operatorname{deg}_{z} b_{12}$. If it does not hold a priori, we can exchange $\sigma_{1}^{[1]}$ and $\sigma_{2}^{[1]}$. 
Again in the case $N^{\text {mon }}=0$ and $\lambda_{1}=\lambda_{2}$, we suppose $\operatorname{deg}_{z} b_{11}<\operatorname{deg}_{z} b_{21}$. If it does not hold a priori, we can replace $\widetilde{s}_{2}$ by a certain linear combination of $\widetilde{s}_{2}$ and $\widetilde{s}_{1}$.

Now $\widetilde{b}_{11}:=z^{-\operatorname{deg}_{z} b_{11}} b_{11} \in \mathbb{C}\{z\}^{*}$ is a unit. Consider $\alpha_{1}:=\beta_{1}+\operatorname{deg}_{z} b_{11}$ and $s_{1}:=z^{\operatorname{deg}_{z} b_{11}} \widetilde{s}_{1}$ $\in C^{\alpha_{1}}$ and the new basis $\left(\sigma_{1}^{[2]}, \sigma_{2}^{[2]}\right)$ of $\mathcal{O}(H)_{0}$ with

$$
\left(\sigma_{1}^{[2]}, \sigma_{2}^{[2]}\right):=\left(\sigma_{1}^{[1]}, \sigma_{2}^{[1]}\right)\left(\begin{array}{cc}
\tilde{b}_{11}^{-1} & -b_{11}^{-1} b_{12} \\
0 & 1
\end{array}\right)=\left(s_{1}, \widetilde{s}_{2}\right)\left(\begin{array}{cc}
1 & 0 \\
\widetilde{b}_{11}^{-1} b_{21} & b_{22}-b_{11}^{-1} b_{12} b_{21}
\end{array}\right) .
$$

Consider $m:=\operatorname{deg}_{z}\left(b_{22}-b_{11}^{-1} b_{12} b_{21}\right) \in \mathbb{Z}\left(+\infty\right.$ is impossible) and $\alpha_{2}:=\beta_{2}+(m-1)$ and $s_{2}:=z^{m-1} \widetilde{s}_{2} \in C^{\alpha_{2}}$. Write $z^{-m+1} \widetilde{b}_{11}^{-1} b_{21}=c_{1}+c_{2}$ with $c_{1} \in \mathbb{C}\left[z^{-1}\right]$ and $c_{2} \in z \mathbb{C}\{z\}$. We can replace $\sigma_{2}^{[2]}$ by $\sigma_{2}^{[3]}:=z s_{2}$ and $\sigma_{1}^{[2]}=s_{1}+\left(c_{1}+c_{2}\right) s_{2}$ by $\sigma_{1}^{[3]}=s_{1}+c_{1} s_{2}$.

(a) Consider the case $N^{\text {mon }}=0$. If $\lambda_{1}=\lambda_{2}$ then $\operatorname{deg}_{z} b_{21} \geq \operatorname{deg}_{z} b_{11}+1$ and thus

$$
\begin{aligned}
\left(c_{1}+c_{2}\right) s_{2} & =\widetilde{b}_{11}^{-1} b_{21} \widetilde{s}_{2} \in \mathbb{C}\{z\} \cdot z^{\operatorname{deg}_{z} b_{21}} \cdot C^{\beta_{2}} \\
& \subset \mathbb{C}\{z\} \cdot z^{\operatorname{deg}_{z} b_{11}+1} \cdot C^{\beta_{2}}=\mathbb{C}\{z\} \cdot C^{\alpha_{1}+1} .
\end{aligned}
$$

In any case (whether $\lambda_{1}=\lambda_{2}$ or $\lambda_{1} \neq \lambda_{2}$ ), we must have $c_{1} \neq 0$. Else the $(T E)$-structure is logarithmic. As the pole has precisely order $2, c_{1}$ is a constant $\neq 0$ (if $\lambda_{1}=\lambda_{2}$, here we need (4.24)), which is now called $t_{2}$. This implies $m-1=\operatorname{deg}_{z} b_{21}$. In the case $N^{\text {mon }}=0$ and $\lambda_{1}=\lambda_{2}$ we have $\beta_{1}=\beta_{2}$ and

$$
\alpha_{2}-\alpha_{1}=m-1-\operatorname{deg}_{z} b_{11}=\operatorname{deg}_{z} b_{21}-\operatorname{deg}_{z} b_{11}>0,
$$

so especially $\alpha_{2} \neq \alpha_{1}$.

(b) Consider the case $N^{\text {mon }} \neq 0$. Then $s_{2}$ is generator of $\operatorname{ker}\left(z \nabla_{\partial_{z}}-\alpha_{2}: C^{\alpha_{2}} \rightarrow C^{\alpha_{2}}\right)$, and we can rescale it such that (4.22) holds. First consider the case $c_{1}=0$. As the pole has precisely order 2, we must have $\alpha_{2}=\alpha_{1}$. Then (4.23) holds with $t_{2}=0$. Now consider the case $c_{1} \neq 0$. Then $\underline{\sigma}^{[3]}=\left(\sigma_{1}^{[3]}, \sigma_{2}^{[3]}\right)$ satisfies

$$
z \nabla_{\partial_{z}} \underline{\sigma}^{[3]}=\underline{\sigma}^{[3]}\left(\begin{array}{cc}
\alpha_{1} & 0 \\
z^{-1}\left(z \partial_{z}-\alpha_{1}+\alpha_{2}\right)\left(c_{1}\right)+z^{\alpha_{1}-\alpha_{2}-1} & \alpha_{2}+1
\end{array}\right) .
$$

First case, $\alpha_{1}-\alpha_{2} \in \mathbb{Z}_{<0}$ : The coefficient of $z^{\alpha_{1}-\alpha_{2}-1}$ in $z^{-1}\left(z \partial_{z}-\alpha_{1}+\alpha_{2}\right)\left(c_{1}\right)+z^{\alpha_{1}-\alpha_{2}-1}$ is 1 . Therefore the pole order is $>2$, a contradiction.

Second case, $\alpha_{1} \geq \alpha_{2}$ : As the pole has precisely order $2, c_{1}$ is a constant $\neq 0$, which is now called $t_{2}$. Then (4.23) holds, and $t_{2} \in \mathbb{C}^{*}$. In the case $\alpha_{1}-\alpha_{2} \in \mathbb{N}, t_{2}$ is obviously independent of the choice of $s_{1}$.

Corollary 4.18 is an immediate consequence of Theorem 4.17.

Corollary 4.18. The set of regular singular, but not logarithmic, rank 2 (TE)-structures over a point is in bijection with the set

$$
\begin{aligned}
\left\{\left(0,\left\{\alpha_{1}, \alpha_{2}\right\}\right) \mid \alpha_{1}, \alpha_{2} \in \mathbb{C}, \alpha_{1} \neq \alpha_{2}\right\} & \cup\left\{\left(1, \alpha_{1}, \alpha_{2}\right) \mid \alpha_{1}=\alpha_{2} \in \mathbb{C}\right\} \\
\cup & \left\{\left(1, \alpha_{1}, \alpha_{2}, t_{2}\right) \mid \alpha_{1}, \alpha_{2} \in \mathbb{C}, \alpha_{1}-\alpha_{2} \in \mathbb{N}, t_{2} \in \mathbb{C}^{*}\right\} .
\end{aligned}
$$

The first set parametrizes the cases with $N^{\mathrm{mon}}=0$, the second and third set parametrize the cases with $N^{\mathrm{mon}} \neq 0$. Theorem 4.17 describes the corresponding (TE)-structures.

Remark 4.19. The connection matrices for the special bases in Theorem 4.17 can be written down easily. 
The basis in (4.20):

$$
\nabla_{z \partial_{z}}\left(s_{1}+t_{2} s_{2}, z s_{2}\right)=\left(s_{1}+t_{2} s_{2}, z s_{2}\right)\left(\begin{array}{cc}
\alpha_{1} & 0 \\
z^{-1}\left(\alpha_{2}-\alpha_{1}\right) t_{2} & \alpha_{2}+1
\end{array}\right) .
$$

The basis in (4.21) with $\widetilde{t}_{2}:=t_{2}^{-1}$ :

$$
\nabla_{z \partial_{z}}\left(s_{2}+\widetilde{t}_{2} s_{1}, z s_{1}\right)=\left(s_{2}+\widetilde{t}_{2} s_{1}, z s_{1}\right)\left(\begin{array}{cc}
\alpha_{2} & 0 \\
z^{-1}\left(\alpha_{1}-\alpha_{2}\right) \widetilde{t}_{2} & \alpha_{1}+1
\end{array}\right) .
$$

The basis in (4.23) with (4.22):

$$
\nabla_{z \partial_{z}}\left(s_{1}+t_{2} s_{2}, z s_{2}\right)=\left(s_{1}+t_{2} s_{2}, z s_{2}\right)\left(\begin{array}{cc}
\alpha_{1} & 0 \\
z^{-1}\left(\alpha_{2}-\alpha_{1}\right) t_{2}+z^{\alpha_{1}-\alpha_{2}-1} & \alpha_{2}+1
\end{array}\right) .
$$

Finally, in the case $N^{\text {mon }} \neq 0$ and $t_{2} \in \mathbb{C}^{*}$, we consider with $\widetilde{t}_{2}:=t_{2}^{-1}$ also the basis $\left(s_{2}+\widetilde{t}_{2} s_{1}, z s_{1}\right)$. Again (4.22) is assumed:

$$
\begin{aligned}
\nabla_{z \partial_{z}}\left(s_{2}+\widetilde{t}_{2} s_{1}, z s_{1}\right)= & \left(s_{2}+\widetilde{t}_{2} s_{1}, z s_{1}\right) \\
& \times\left(\begin{array}{cc}
\alpha_{2}+z^{\alpha_{1}-\alpha_{2}} \widetilde{t}_{2} & z^{\alpha_{1}-\alpha_{2}+1} \\
z^{-1}\left(\alpha_{1}-\alpha_{2}\right) \widetilde{t}_{2}-z^{\alpha_{1}-\alpha_{2}-1} \widetilde{t}_{2}^{2} & \alpha_{1}+1-z^{\alpha_{1}-\alpha_{2}} \widetilde{t_{2}}
\end{array}\right) .
\end{aligned}
$$

\subsection{The case $(\mathrm{Log})$ with $\operatorname{tr} \mathcal{U}=0$}

The $(T E)$-structures over a point of the type $(\log )$ with $\operatorname{tr} \mathcal{U}=0$ are the logarithmic $(T E)$ structures over a point. Just as the regular singular $(T E)$-structures, they can easily be classified using elementary sections. Theorem 4.20 splits them into two cases. We use again the Notation 4.16 .

Theorem 4.20. Consider a logarithmic rank $2(T E)$-structure $(H \rightarrow \mathbb{C}, \nabla)$ over a point. Associate to it the data in the Notation 4.16.

(a) The case $N^{\mathrm{mon}}=0$ : There exist unique numbers $\alpha_{1}, \alpha_{2}$ with $\mathrm{e}^{-2 \pi \mathrm{i} \alpha_{j}}=\lambda_{j}$ and the following property: There exist elementary sections $s_{1} \in C^{\alpha_{1}} \backslash\{0\}$ and $s_{2} \in C^{\alpha_{2}} \backslash\{0\}$ such that

$$
\mathcal{O}(H)_{0}=\mathbb{C}\{z\} s_{1} \oplus \mathbb{C}\{z\} s_{2} .
$$

The isomorphism class of the (TE)-structure is uniquely determined by the information $N^{\text {mon }}=0$ and the set $\left\{\alpha_{1}, \alpha_{2}\right\}$. The numbers $\alpha_{1}$ and $\alpha_{2}$ are called leading exponents.

(b) The case $N^{\text {mon }} \neq 0$ (thus $\lambda_{1}=\lambda_{2}$ ): There exist unique numbers $\alpha_{1}, \alpha_{2}$ with $\mathrm{e}^{-2 \pi \mathrm{i} \alpha_{j}}=\lambda_{1}$ and $\alpha_{1}-\alpha_{2} \in \mathbb{N}_{0}$ and the following properties: Choose any elementary section $s_{1} \in$ $C^{\alpha_{1}}-\operatorname{ker}\left(\nabla_{z \partial_{z}}-\alpha_{1}: C^{\alpha_{1}} \rightarrow C^{\alpha_{1}}\right)$. The elementary section $s_{2} \in C^{\alpha_{2}}$ with

$$
\left(z \nabla_{\partial_{z}}-\alpha_{1}\right)\left(s_{1}\right)=z^{\alpha_{1}-\alpha_{2}} s_{2}
$$

is a generator of $\operatorname{ker}\left(z \nabla_{\partial_{z}}-\alpha_{2}: C^{\alpha_{2}} \rightarrow C^{\alpha_{2}}\right)$. Then

$$
\mathcal{O}(H)_{0}=\mathbb{C}\{z\} s_{1} \oplus \mathbb{C}\{z\} s_{2} .
$$

The isomorphism class of the (TE)-structure is uniquely determined by the information $N^{\text {mon }} \neq 0$ and the set $\left\{\alpha_{1}, \alpha_{2}\right\}$. The numbers $\alpha_{1}$ and $\alpha_{2}$ are called leading exponents. 
Proof. First, $(a)$ and $(b)$ are considered together. By Theorem 3.23(a), $\mathcal{O}(H)_{0}$ is generated by two elementary sections $s_{1} \in C^{\alpha_{1}}$ and $s_{2} \in C^{\alpha_{2}}$ for some numbers $\alpha_{1}$ and $\alpha_{2}$. The numbers $\alpha_{1}$ and $\alpha_{2}$ are the eigenvalues of the residue endomorphism. So, they are unique. This finishes already the proof of part $(a)$.

(b) Consider the case $N^{\text {mon }} \neq 0$. We can renumber $s_{1}$ and $s_{2}$ if necessary, so that afterwards $\alpha_{1}-\alpha_{2} \in \mathbb{N}_{0}$. If $\alpha_{1}=\alpha_{2}$, then $\mathcal{O}(H)_{0}=\mathbb{C}\{z\} C^{\alpha_{1}}$, and $s_{1}$ and $s_{2}$ can be changed so that $s_{1} \in C^{\alpha_{1}} \backslash \operatorname{ker}\left(\nabla_{z \partial_{z}}-\alpha_{1}\right)$ and $s_{2} \in \operatorname{ker}\left(\nabla_{z \partial_{z}}-\alpha_{1}: C^{\alpha_{1}} \rightarrow C^{\alpha_{1}}\right) \backslash\{0\}$ satisfy (4.30). Then nothing more has to be shown. Consider the case $\alpha_{1}-\alpha_{2} \in \mathbb{N}$. If $s_{2} \in C^{\alpha_{2}} \backslash \operatorname{ker}\left(\nabla_{z \partial_{z}}-\alpha_{2}\right)$, then $\left(\nabla_{z \partial_{z}}-\alpha_{2}\right)\left(s_{2}\right)$ is not in $\mathcal{O}(H)_{0}$, and thus the pole is not logarithmic, a contradiction. Therefore $s_{2} \in \operatorname{ker}\left(\nabla_{z \partial_{z}}-\alpha_{2}: C^{\alpha_{2}} \rightarrow C^{\alpha_{2}}\right)$. Then necessarily $s_{1} \in C^{\alpha_{1}} \backslash \operatorname{ker}\left(\nabla_{z \partial_{z}}-\alpha_{1}: C^{\alpha_{1}} \rightarrow C^{\alpha_{1}}\right)$. We can rescale $s_{2}$ so that (4.30) holds. Nothing more has to be shown.

Corollary 4.21 is an immediate consequence of Theorem 4.20.

Corollary 4.21. The set of logarithmic rank 2 (TE)-structures over a point is in bijection with the set

$$
\left\{\left(0,\left\{\alpha_{1}, \alpha_{2}\right\}\right) \mid \alpha_{1}, \alpha_{2} \in \mathbb{C},\right\} \cup\left\{\left(1, \alpha_{1}, \alpha_{2}\right) \mid \alpha_{1}, \alpha_{2} \in \mathbb{C}, \alpha_{1}-\alpha_{2} \in \mathbb{N}_{0}\right\}
$$

The first set parametrizes the cases with $N^{\mathrm{mon}}=0$, the second set parametrizes the cases with $N^{\text {mon }} \neq 0$. Theorem 4.20 describes the corresponding $(T E)$-structures.

Remark 4.22. The connection matrices for the special bases in Theorem 4.20 can be written down easily.

The basis in (4.29):

$$
\nabla_{z \partial_{z}}\left(s_{1}, s_{2}\right)=\left(s_{1}, s_{2}\right)\left(\begin{array}{cc}
\alpha_{1} & 0 \\
0 & \alpha_{2}
\end{array}\right)
$$

The basis in (4.31):

$$
\nabla_{z \partial_{z}}\left(s_{1}, s_{2}\right)=\left(s_{1}, s_{2}\right)\left(\begin{array}{cc}
\alpha_{1} & 0 \\
z^{\alpha_{1}-\alpha_{2}} & \alpha_{2}
\end{array}\right)
$$

The basis $\left(s_{1}, s_{2}\right)$ gives a Birkhoff normal form in the cases $N^{\text {mon }}=0$ and in the cases $\left(N^{\text {mon }} \neq 0\right.$ and $\left.\alpha_{1}=\alpha_{2}\right)$. In the cases $\left(N^{\text {mon }} \neq 0\right.$ and $\left.\alpha_{1}-\alpha_{2} \in \mathbb{N}\right)$, a Birkhoff normal form does not exist.

\section{Rank 2 ( $T E$ )-structures over germs of regular $F$-manifolds}

This section discusses unfoldings of $(T E)$-structures over a point $t^{0}$ of type (Sem) or (Bra) or (Reg). Here Malgrange's unfolding result Theorem 3.16(c) applies. It provides a universal unfolding for the $(T E)$-structure over $t^{0}$. Any unfolding is induced by the universal unfolding. The universal unfoldings turn out to be precisely the $(T E)$-structures with primitive Higgs fields over germs of regular $F$-manifolds.

Sections 6 and 8 discuss unfoldings of (TE)-structures over a point of type (Log). Section 8 treats arbitrary such unfoldings. Section 6 prepares this. It treats 1-parameter unfoldings with trace free pole parts of logarithmic $(T E)$-structures over a point.

If one starts with a $(T E)$-structure with primitive Higgs field over a germ $\left(M, t^{0}\right)$ of a regular $F$-manifold, then the endomorphism $\left.\mathcal{U}\right|_{t^{0}}: K_{t^{0}} \rightarrow K_{t^{0}}$ is regular.

Vice versa, if one starts with a $(T E)$-structure over a point $t^{0}$ with a regular endomorphism $\mathcal{U}: K_{t^{0}} \rightarrow K_{t^{0}}$, then it unfolds uniquely to a $(T E)$-structure with primitive Higgs field over 
a germ of a regular F-manifold by Malgrange's result Theorem 3.16(c). The germ of the regular $F$-manifold is uniquely determined by the isomorphism class of $\mathcal{U}: K_{t^{0}} \rightarrow K_{t^{0}}$ (i.e., its Jordan block structure). And the (TE)-structure is uniquely determined by its restriction to $t^{0}$.

The following statement on the rank 2 cases is an immediate consequence of Malgrange's unfolding result Theorem 3.16(c), the classification of germs of regular 2-dimensional $F$-manifolds in Remark 2.6(ii) (building on Theorems 2.2 and 2.3, see also Remark 3.17(iii)) and the classification of the rank 2 (TE)-structures into the cases (Sem), (Bra), (Reg) and (Log) in Definition 4.4 .

\section{Corollary 5.1.}

(a) For any rank 2 (TE)-structure over a point $t^{0}$ except those of type (Log), the endomorphism $\mathcal{U}: K_{t^{0}} \rightarrow K_{t^{0}}$ is regular. The (TE)-structure has a unique universal unfolding. This unfolding has a primitive Higgs field. Its base space is a germ $\left(M, t^{0}\right)=\left(\mathbb{C}^{2}, 0\right)$ of an F-manifold with Euler field and is as follows:

\begin{tabular}{c|c|c}
\hline Type & F-manifold & Euler field \\
\hline$($ Sem $)$ & $A_{1}^{2}$ & $\sum_{i=1}^{2}\left(u_{i}+c_{i}\right) e_{i}$ with $c_{1} \neq c_{2}$ \\
$($ Bra $)$ or $($ Reg $)$ & $\mathcal{N}_{2}$ & $t_{1} \partial_{1}+g\left(t_{2}\right) \partial_{2}$ with $g(0) \neq 0$ \\
\hline
\end{tabular}

In the case of (Bra) or (Reg), a coordinate change brings $E$ to the form $t_{1} \partial_{1}+\partial_{2}$.

(b) Any unfolding of a rank 2 (TE)-structure over $t^{0}$ with regular endomorphism $\mathcal{U}: K_{t^{0}} \rightarrow K_{t^{0}}$ is induced by the universal unfolding in $(a)$.

Because of the existence and uniqueness of the universal unfolding, it is not really necessary to give it explicitly. On the other hand, in rank 2, it is easy to give it explicitly. The following lemma offers one way.

Lemma 5.2. Let $(H \rightarrow \mathbb{C}, \nabla)$ by a $(T E)$-structure over a point with monodromy $M^{\text {mon }}$ of some rank $r \in \mathbb{N}$. It has an unfolding which is a $(T E)$-structure $\left(H^{(\mathrm{unf})} \rightarrow \mathbb{C} \times M, \nabla\right)$, where $M=\mathbb{C} \times \mathbb{C}^{*}$ with coordinates $t=\left(t_{1}, t_{2}\right)\left(\right.$ on $\left.\mathbb{C}^{2} \supset M\right)$, with the following properties.

(a) The monodromy around $t_{2}=0$ is $\left(M^{\mathrm{mon}}\right)^{-1}$.

(b) The original (TE)-structure is isomorphic to the one over $t^{0}=(0,1)$.

(c) If $\underline{v}^{0}$ is a $\mathbb{C}\{z\}$-basis of $\mathcal{O}(H)_{0}$ with $z^{2} \nabla_{\partial_{z}} \underline{v}^{0}=\underline{v}^{0} B^{0}$, then $H^{(\text {unf })}$ has over $(\mathbb{C}, 0) \times M$ a basis $\underline{v}$ such that the matrices $A_{1}, A_{2}$ and $B$ in (3.2)-(3.4) are as follows

$$
\begin{aligned}
& A_{1}=C_{1}, \\
& A_{2}=-B^{0}\left(\frac{z}{t_{2}}\right) \\
& B=-t_{1} C_{1}+t_{2} B^{0}\left(\frac{z}{t_{2}}\right)=-t_{1} A_{1}-t_{2} A_{2} .
\end{aligned}
$$

(d) If $\left.\mathcal{U}\right|_{t^{0}}$ is regular and $\operatorname{rank} H=2$, then the Higgs field of the (TE)-structure $H^{(\mathrm{unf})}$ is everywhere primitive. Therefore then $M$ is an F-manifold with Euler field. The Euler field is $E=t_{1} \partial_{1}+t_{2} \partial_{2}$.

(e) If $\left.\mathcal{U}\right|_{t^{0}}$ is regular and $\operatorname{rank} H=2$, the $(T E)$-structure over the germ $\left(M, t^{0}\right)$ is the universal unfolding of the one over $t^{0}$. 
Proof. Let $\underline{f}^{0}=\left(f_{1}^{0}, \ldots, f_{r}^{0}\right)$ be a flat multivalued basis of $H^{\prime}:=\left.H\right|_{\mathbb{C}^{*}}$. Let $M^{\text {mat }} \in \mathrm{GL}_{r}(\mathbb{C})$ be the matrix of its monodromy, so $\underline{f}^{0}\left(z \mathrm{e}^{2 \pi \mathrm{i}}\right)=\underline{f}^{0} \cdot M^{\text {mat }}$. Let $\underline{v}^{0}=\left(v_{1}^{0}, \ldots, v_{r}^{0}\right)$ be a $\mathbb{C}\{z\}$ basis of $\mathcal{O}(H)_{0}$. Let $B^{0} \in \mathrm{GL}_{r}(\mathbb{C}\{\bar{z}\})$ be the matrix with $z^{2} \nabla_{\partial_{z}} \underline{v}^{0}=\underline{v}^{0} B^{0}$. Consider the matrix $\Psi(z, t)$ with multivalued entries with

$$
\underline{v}^{0}=\underline{f}^{0} \cdot \Psi(z) .
$$

Then

$$
\begin{aligned}
& \Psi\left(z \mathrm{e}^{2 \pi \mathrm{i}}\right)=\left(M^{\mathrm{mat}}\right)^{-1} \cdot \Psi(z), \\
& \Psi^{-1} \partial_{z} \Psi=z^{-2} B^{0}(z) .
\end{aligned}
$$

Embed the flat bundle $H^{\prime}:=\left.H\right|_{\mathbb{C}^{*}}$ as the bundle over $t^{0}=(0,1)$ into a flat bundle $H^{(m f)^{\prime}} \rightarrow$ $\mathbb{C}^{*} \times M$ with monodromy $M^{\text {mon }}$ around $z=0$ and monodromy $\left(M^{\text {mon }}\right)^{-1}$ around $t_{2}=0$. The flat multivalued basis $\underline{f}^{0}$ of $H^{\prime}$ extends to a flat multivalued basis $\underline{f}$ of $H^{(m f)^{\prime}}$ with

$$
\begin{aligned}
& \underline{f}\left(z \mathrm{e}^{2 \pi \mathrm{i}}, t\right)=\underline{f}(z, t) M^{\mathrm{mat}}, \\
& \underline{f}\left(z, t_{1}, t_{2} \mathrm{e}^{2 \pi \mathrm{i}}\right)=\underline{f}(z, t)\left(M^{\mathrm{mat}}\right)^{-1} .
\end{aligned}
$$

The tuple of sections $\underline{v}$ with

$$
\underline{v}=\underline{f} \cdot \mathrm{e}^{t_{1} / z} \Psi\left(\frac{z}{t_{2}}\right)
$$

is univalued, it is a basis of $H^{(m f)^{\prime}}$ in a neighbourhood of $\{0\} \times M$, and it has the connection matrices in (5.1)-(5.3): The calculations for $A_{2}$ and $B$ are

$$
\begin{aligned}
\nabla_{\partial_{2} \underline{v}} & =\underline{f} \mathrm{e}^{t_{1} / z}\left(-\frac{z}{t_{2}^{2}}\right)\left(\partial_{z} \Psi\right)\left(\frac{z}{t_{2}}\right)=\underline{f} \mathrm{e}^{t_{1} / z}\left(-\frac{z}{t_{2}^{2}}\right) \Psi\left(\frac{z}{t_{2}}\right)\left(\frac{z}{t_{2}}\right)^{-2} B^{0}\left(\frac{z}{t_{2}}\right) \\
& =\underline{v}\left(-\frac{1}{z}\right) B^{0}\left(\frac{z}{t_{2}}\right), \\
\nabla_{\partial_{z} \underline{v}} & =\underline{f} \mathrm{e}^{t_{1} / z}\left(\left(-\frac{t_{1}}{z^{2}}\right) \Psi\left(\frac{z}{t_{2}}\right)+\left(\frac{1}{t_{2}}\right)\left(\partial_{z} \Psi\right)\left(\frac{z}{t_{2}}\right)\right) \\
& =\underline{f} \mathrm{e}^{t_{1} / z}\left(\left(-\frac{t_{1}}{z^{2}}\right) \Psi\left(\frac{z}{t_{2}}\right)+\left(\frac{1}{t_{2}}\right) \Psi\left(\frac{z}{t_{2}}\right)\left(\frac{z}{t_{2}}\right)^{-2} B^{0}\left(\frac{z}{t_{2}}\right)\right) \\
& =\underline{v}\left(\left(-\frac{t_{1}}{z^{2}}\right) C_{1}+\left(\frac{t_{2}}{z^{2}}\right) B^{0}\left(\frac{z}{t_{2}}\right)\right) .
\end{aligned}
$$

Therefore $\underline{v}$ defines a $(T E)$-structure, which we call $\left(H^{(\text {unf })} \rightarrow \mathbb{C} \times M, \nabla\right)$. It unfolds the one over $t^{0}=(0,1)$, and that one is isomorphic to $(H \rightarrow \mathbb{C}, \nabla)$.

It rests to show $(d)$ and $(e)$. Suppose $\operatorname{rank} H=2$. Then $\left.\mathcal{U}\right|_{t^{0}}$ is regular if and only if $\left(B^{0}\right)^{(0)} \notin \mathbb{C} \cdot C_{1}$. Then also $A_{2}^{(0)}(t)=-\left(B^{0}\right)^{(0)} \notin \mathbb{C} \cdot C_{1}$, so then the Higgs field of the $(T E)$ structure $H^{(\text {unf })}$ is everywhere primitive. Because of $B^{(0)}=-t_{1} A_{1}^{(0)}-t_{2} A_{2}^{(0)}$, the Euler field is $E=t_{1} \partial_{1}+t_{2} \partial_{2}$. (e) follows from $(d)$ and Malgrange's result Theorem 3.16(c).

\section{Remarks 5.3.}

(i) In the cases (Reg) we will see the universal unfoldings again in Section 7, in Remarks 7.2. In a first step in Remarks 7.1, the value $t_{2}$ in the normal form in Remarks 4.19 is turned into a parameter in $\mathbb{P}^{1}$. Remarks 7.2 add another parameter $t_{1}$ in $\mathbb{C}$. Then the Higgs field becomes primitive and the base space $\mathbb{C} \times \mathbb{P}^{1}$ becomes a 2-dimensional $F$-manifold with Euler field. For each $t^{0} \in \mathbb{C} \times \mathbb{C}^{*}$, the $(T E)$-structure over $t^{0}$ is of type (Reg), and the $(T E)$-structure over the germ $\left(M, t^{0}\right)$ is a universal unfolding of the one over $t^{0}$. 
(ii) In the cases (Bra), the following formulas give a universal unfolding over $\left(\mathbb{C}^{2}, 0\right)$ of any $(T E)$-structure of type (Bra) over the point 0 (see Theorem 4.11 for their classification), such that the Euler field is $E=\left(t_{1}+c_{1}\right) \partial_{1}+\partial_{2}$. Here $\rho^{(1)} \in \mathbb{C}, b_{3}^{(0)} \in \mathbb{C}, b_{2}^{(0)}, b_{4}^{(1)} \in \mathbb{C}^{*}$,

$$
\begin{aligned}
A_{1} & =C_{1} \\
A_{2} & =-b_{2}^{(0)} C_{2}-z\left(\frac{1}{2}+b_{3}^{(1)}\right) D-z b_{4}^{(1)} \mathrm{e}^{t_{2}} E \\
B & =\left(-t_{1}-c_{1}\right) C_{1}+b_{2}^{(0)} C_{2}+z\left(\rho^{(1)} C_{1}+b_{3}^{(1)} D+b_{4}^{(1)} \mathrm{e}^{t_{2}} E\right) \\
& =\left(-t_{1}-c_{1}\right) A_{1}-A_{2}+z \rho^{(1)} C_{1}-z \frac{1}{2} D .
\end{aligned}
$$

(iii) In the cases (Sem), a (TE)-structure over a point extends uniquely to a (TE)-structure over the universal covering $M$ of the manifold $\left\{\left(u_{1}, u_{2}\right) \in \mathbb{C}^{2} \mid u_{1} \neq u_{2}\right\}$ (see [17] and [21, Chapter III, Theorem 2.10]). For each $t^{0} \in M$ the (TE)-structure over $t^{0}$ is of type (Sem), and the (TE)-structure over the germ $\left(M, t^{0}\right)$ is the universal unfolding of the $(T E)$-structure over $t^{0}$.

\section{1-parameter unfoldings of logarithmic ( $T E$ )-structures over a point}

This section classifies unfoldings over $\left(M, t^{0}\right)=(\mathbb{C}, 0)$ with trace free pole part of logarithmic (TE)-structures over the point $t^{0}$.

It is a preparation for Section 8, which treats arbitrary unfoldings of $(T E)$-structures of type ( $\log )$ over a point.

Section 6.1: An unfolding with trace free pole part over $\left(M, t^{0}\right)=(\mathbb{C}, 0)$ of a logarithmic rank $2(T E)$-structure over $t^{0}$ will be considered. Invariants of it will be defined. Theorem 6.2 gives constraints on these invariants and shows that the monodromy is semisimple if the generic type is (Sem) or (Bra).

By Theorem 3.20(a) (which is trivial in our case because of the logarithmic pole at $z=0$ of the (TE)-structure over $\left.t^{0}\right)$ and Remark 3.19(iii), the (TE)-structure has a Birkhoff normal form, i.e., an extension to a pure (TLE)-structure, if its monodromy is semisimple.

Section 6.2: All pure (TLE)-structures over $\left(M, t^{0}\right)=(\mathbb{C}, 0)$ with trace free pole part and with logarithmic restriction to $t^{0}$ are classified in Theorem 6.3. These comprise all with semisimple monodromy and thus all with generic types (Sem) or (Bra).

Section 6.3: All $(T E)$-structures over $\left(M, t^{0}\right)=(\mathbb{C}, 0)$ with trace free pole part and with logarithmic restriction over $t^{0}$ whose monodromies have a $2 \times 2$ Jordan block are classified in Theorem 6.7. Their generic types are (Reg) or ( $\mathrm{Log})$ because of Theorem 6.2. Most of them have no Birkhoff normal forms. The intersection with Theorem 6.3 is small and consists of those which have Birkhoff normal forms.

Theorems 6.3 and 6.7 together give all unfoldings with trace free pole parts over $\left(M, t^{0}\right)=$ $(\mathbb{C}, 0)$ of logarithmic rank $2(T E)$-structures over $t^{0}$.

\subsection{Numerical invariants for such $(T E)$-structures}

The next definition gives some numerical invariants for such $(T E)$-structures. Recall the invariants $\delta^{(0)}$ and $\delta^{(1)}$ in Lemma 3.9.

Definition 6.1. Let $\left(H \rightarrow \mathbb{C} \times\left(M, t^{0}\right), \nabla\right)$ be a $(T E)$-structure with trace free pole part over $\left(M, t^{0}\right)=(\mathbb{C}, 0)$ (with coordinate $t$ ) whose restriction over $t^{0}=0$ is logarithmic. Let $M \subset \mathbb{C}$ be a neighborhood of 0 on which the $(T E)$-structure is defined. On $M \backslash\{0\}$ it has a fixed 
type, (Sem) or (Bra) or (Reg) or (Log), which is called the generic type of the (TE)-structure. Lemma 4.3 characterizes the generic type in terms of (non) vanishing of $\delta^{(0)}, \delta^{(1)} \in t \mathbb{C}\{t\}$ and $\mathcal{U}$ :

\begin{tabular}{c|c|c|c}
\hline$($ Sem $)$ & $($ Bra $)$ & $($ Reg $)$ & $(\log )$ \\
\hline$\delta^{(0)} \neq 0$ & $\delta^{(0)}=0, \delta^{(1)} \neq 0$ & $\delta^{(0)}=\delta^{(1)}=0, \mathcal{U} \neq 0$ & $\mathcal{U}=0$ \\
\hline
\end{tabular}

For the generic types (Sem), (Bra) and (Reg), define $k_{1} \in \mathbb{N}$ by

$$
k_{1}:=\max \left(k \in \mathbb{N} \mid \mathcal{U}\left(\mathcal{O}(H)_{0}\right) \subset t^{k} \mathcal{O}(H)_{0} .\right.
$$

For the generic types (Sem) and (Bra) define $k_{2} \in \mathbb{Z}$ by

$$
k_{2}:= \begin{cases}\operatorname{deg}_{t} \delta^{(0)}-k_{1} & \text { for the generic type (Sem) }, \\ \operatorname{deg}_{t} \delta^{(1)}-k_{1} & \text { for the generic type (Bra). }\end{cases}
$$

The following theorem gives for the generic type (Bra) and part of the generic type (Sem) restrictions on the eigenvalues of the residue endomorphism of the logarithmic pole at $z=0$ of the $(T E)$-structure over $t^{0}=0$. And it shows that the monodromy is semisimple if the generic type is (Sem) or (Bra).

Theorem 6.2. Let $\left(H \rightarrow \mathbb{C} \times\left(M, t^{0}\right), \nabla\right)$ be a rank $2(T E)$-structure with trace free pole part over $\left(M, t^{0}\right)=(\mathbb{C}, 0)$ whose restriction over $t^{0}=0$ is logarithmic. Recall the invariant $\rho^{(1)} \in \mathbb{C}$ from Lemma $3.9(b)$, and recall the invariants $k_{1} \in \mathbb{N}$ and $k_{2} \in \mathbb{Z}$ from Definition 6.1 if the generic type is $(\mathrm{Sem})$ or (Bra).

(a) Suppose that the generic type is (Sem).

(i) Then $k_{2} \geq k_{1}$.

(ii) If $k_{2}>k_{1}$ then the eigenvalues of the residue endomorphism of the logarithmic pole at $z=0$ of the (TE)-structure over $t^{0}$ are $\rho^{(1)} \pm \frac{k_{1}-k_{2}}{2\left(k_{1}+k_{2}\right)}$. Their difference is smaller than 1. Especially, the eigenvalues of the monodromy are different, and the monodromy is semisimple.

(iii) Also if $k_{1}=k_{2}$, the monodromy is semisimple.

(b) Suppose that the generic type is (Bra).

(i) Then $k_{2} \in \mathbb{N}$.

(ii) The eigenvalues of the residue endomorphism of the logarithmic pole at $z=0$ of the $(T E)$-structure over $t^{0}$ are $\rho^{(1)} \pm \frac{-k_{2}}{2\left(k_{1}+k_{2}\right)}$. Their difference is smaller than 1. Especially, the eigenvalues of the monodromy are different, and the monodromy is semisimple.

Proof. By Lemma 3.11, a $\mathbb{C}\{t, z\}$-basis $\underline{v}$ of the germ $\mathcal{O}(H)_{(0,0)}$ can be chosen such that the matrices $A$ and $B \in M_{2 \times 2}(\mathbb{C}\{t, z\})$ with $z \nabla_{\partial_{t}} \underline{v}=\underline{v} A$ and $z^{2} \nabla_{\partial_{z}} \underline{v}=\underline{v} B$ satisfy $(3.14), 0=$ $\operatorname{tr} A=\operatorname{tr}\left(B-z \rho^{(1)} C_{1}\right)$, or, more explicitly,

$$
\begin{aligned}
& A=a_{2} C_{2}+a_{3} D+a_{4} E \quad \text { with } \quad a_{2}, a_{3}, a_{4} \in \mathbb{C}\{t, z\}, \\
& B=z \rho^{(1)} C_{1}+b_{2} C_{2}+b_{3} D+b_{4} E \quad \text { with } \quad b_{2}, b_{3}, b_{4} \in \mathbb{C}\{t, z\} .
\end{aligned}
$$

Write $a_{j}=\sum_{k \geq 0} a_{j}^{(k)} z^{k}$ and $a_{j}^{(k)}=\sum_{l \geq 0} a_{j, l}^{(k)} t^{l} \in \mathbb{C}\{t\}$, and analogously for $b_{j}$. Condition (3.8) says here

$$
0=z \partial_{t} B-z^{2} \partial_{z} A+z A+[A, B]
$$




$$
\begin{aligned}
= & C_{2}\left[z \partial_{t} b_{2}+z a_{2}^{(0)}-\sum_{k \geq 2}(k-1) a_{2}^{(k)} z^{k+1}+2 a_{2} b_{3}-2 a_{3} b_{2}\right] \\
& +D\left[z \partial_{t} b_{3}+z a_{3}^{(0)}-\sum_{k \geq 2}(k-1) a_{3}^{(k)} z^{k+1}-a_{2} b_{4}+a_{4} b_{2}\right] \\
& +E\left[z \partial_{t} b_{4}+z a_{4}^{(0)}-\sum_{k \geq 2}(k-1) a_{4}^{(k)} z^{k+1}-2 a_{4} b_{3}+2 a_{3} b_{4}\right] .
\end{aligned}
$$

(a) Suppose that the generic type is (Sem).

(i) By definition of $k_{1}$ and $k_{2}$,

$$
\begin{aligned}
& k_{1}=\min \left(\operatorname{deg}_{t} b_{2}^{(0)}, \operatorname{deg}_{t} b_{3}^{(0)}, \operatorname{deg}_{t} b_{4}^{(0)}\right), \\
& k_{1}+k_{2}=\operatorname{deg}_{t}\left(\left(b_{3}^{(0)}\right)^{2}+b_{2}^{(0)} b_{4}^{(0)}\right) \geq 2 k_{1},
\end{aligned}
$$

thus $k_{2} \geq k_{1}$.

(ii) Suppose $k_{2}>k_{1}$. By a linear change of the basis $\underline{v}$, we can arrange that $k_{1}=\operatorname{deg}_{t} b_{2}^{(0)}$. The base change matrix $T=C_{1}+b_{3}^{(0)} / b_{2}^{(0)} \cdot E \in \mathrm{GL}_{2}(\mathbb{C}\{t\})$ gives the new basis $\underline{\widetilde{v}}=\underline{v} \cdot T$ with matrix

$$
\widetilde{B}^{(0)}=T^{-1} B^{(0)} T=b_{2}^{(0)} C_{2}+\left(b_{4}^{(0)}+\frac{\left(b_{3}^{(0)}\right)^{2}}{b_{2}^{(0)}}\right) E .
$$

We can make a coordinate change in $t$ such that afterwards

$$
b_{2}^{(0)} b_{4}^{(0)}+\left(b_{3}^{(0)}\right)^{2}=\gamma^{2} t^{k_{1}+k_{2}}
$$

for an arbitrarily chosen $\gamma \in \mathbb{C}^{*}$. Then a diagonal base change leads to a basis which is again called $\underline{v}$ with matrices which are again called $A$ and $B$ with

$$
b_{3}^{(0)}=0, \quad b_{2}^{(0)}=\gamma t^{k_{1}}, \quad b_{4}^{(0)}=\gamma t^{k_{2}} .
$$

Now the vanishing of the coefficients in $\mathbb{C}\{t\}$ of $C_{2} \cdot z^{0}, C_{2} \cdot z^{1}, D \cdot z^{0}, D \cdot z^{1}$ and $E \cdot z^{1}$ in (6.2)-(6.4) tells the following:

$$
\begin{aligned}
C_{2} \cdot z^{0}: & a_{3}^{(0)}=0 \\
C_{2} \cdot z^{1}: & 0=k_{1} \gamma t^{k_{1}-1}+a_{2}^{(0)}\left(1+2 b_{3}^{(1)}\right)-2 a_{3}^{(1)} \gamma t^{k_{1}}, \\
& \text { so } \quad \operatorname{deg}_{t} a_{2}^{(0)}=k_{1}-1, \quad 0=k_{1} \gamma+a_{2, k_{1}-1}^{(0)}\left(1+2 b_{3,0}^{(1)}\right), \\
D \cdot z^{0}: \quad & a_{2}^{(0)} \gamma t^{k_{2}}=a_{4}^{(0)} \gamma t^{k_{1}}, \quad \text { so } \quad a_{4}^{(0)}=a_{2}^{(0)} t^{k_{2}-k_{1}}, \\
& \text { so } \quad \operatorname{deg} a_{4}^{(0)}=k_{2}-1, \quad \text { and } \quad a_{4, k_{2}-1}^{(0)}=a_{2, k_{1}-1}^{(0)} . \\
D \cdot z^{1}: \quad & a_{2}^{(0)} b_{4}^{(1)}+a_{2}^{(1)} \gamma t^{k_{2}}=a_{4}^{(0)} b_{2}^{(1)}+a_{4}^{(1)} \gamma t^{k_{1}}, \\
& \text { so } \quad b_{4,0}^{(1)}=0 \quad\left(\text { here } k_{2}>k_{1}\right. \text { is used), } \\
E \cdot z^{1}: & 0=k_{2} \gamma t^{k_{2}-1}+a_{4}^{(0)}\left(1-2 b_{3}^{(1)}\right)+2 a_{3}^{(1)} \gamma t^{k_{2}}, \\
& \text { so } 0=k_{2} \gamma+a_{4, k_{2}-1}^{(0)}\left(1-2 b_{3,0}^{(1)}\right) .
\end{aligned}
$$

This shows

$$
b_{4,0}^{(1)}=0, \quad b_{3,0}^{(1)}=\frac{k_{1}-k_{2}}{2\left(k_{1}+k_{2}\right)} \in\left(-\frac{1}{2}, 0\right) \cap \mathbb{Q} .
$$


With respect to the basis $\left.\underline{v}\right|_{(0,0)}$ of $K_{(0,0)}$, the matrix of the residue endomorphism of the logarithmic pole at $z=0$ of the $(T E)$-structure over $t^{0}=0$ is

$$
B^{(1)}(0)=\rho^{(1)} C_{1}+b_{3,0}^{(1)} D+b_{2,0}^{(1)} C_{2} .
$$

It is semisimple with the eigenvalues $\rho^{(1)} \pm b_{3,0}^{(1)}$, whose difference is smaller than 1 . The monodromy is semisimple with the two different eigenvalues $\exp \left(-2 \pi \mathrm{i}\left(\rho^{(1)} \pm b_{3,0}^{(1)}\right)\right)$.

(iii) Suppose $k_{2}=k_{1}$. As in the proof of $(i i)$, we can make a coordinate change in $t$ and then obtain a $\mathbb{C}\{t, z\}$-basis $\underline{\widetilde{v}}$ of $\mathcal{O}(H)_{(0,0)}$ with

$$
\widetilde{b}_{3}^{(0)}=0, \quad \widetilde{b}_{2}^{(0)}=\widetilde{b}_{4}^{(0)}=\gamma t^{k_{1}}
$$

for an arbitrarily chosen $\gamma \in \mathbb{C}^{*}$. Now the constant base change matrix $T=\left(\begin{array}{ll}1 & 1 \\ 1 & -1\end{array}\right)$ gives the basis $\underline{v}=\underline{\widetilde{v}} \cdot T$ with

$$
b_{2}^{(0)}=b_{4}^{(0)}=0, \quad b_{3}^{(0)}=\gamma t^{k_{1}} .
$$

The vanishing of the coefficients in $\mathbb{C}\{t\}$ of $C_{2} \cdot z^{0}, E \cdot z^{0}, D \cdot z^{1}, C_{2} \cdot z^{1}$ and $E \cdot z^{1}$ in (6.2)-(6.4) tells the following:

$$
\begin{array}{ll}
C_{2} \cdot z^{0}: & a_{2}^{(0)}=0, \\
E \cdot z^{0}: & a_{4}^{(0)}=0, \\
D \cdot z^{1}: & 0=k_{1} \gamma t^{k_{1}-1}+a_{3}^{(0)}, \quad \text { so } \quad a_{3}^{(0)}=-k_{1} \gamma t^{k_{1}-1}, \\
C_{2} \cdot z^{1}: & b_{2}^{(1)}=\frac{b_{3}^{(0)}}{a_{3}^{(0)}} a_{2}^{(1)}=\frac{-1}{k_{1}} \cdot t \cdot a_{2}^{(1)}, \quad \text { so } \quad b_{2,0}^{(1)}=0, \\
E \cdot z^{1}: & b_{4}^{(1)}=\frac{b_{3}^{(0)}}{a_{3}^{(0)}} a_{4}^{(1)}=\frac{-1}{k_{1}} \cdot t \cdot a_{4}^{(1)}, \quad \text { so } \quad b_{4,0}^{(1)}=0 .
\end{array}
$$

With respect to the basis $\left.\underline{v}\right|_{(0,0)}$ of $K_{(0,0)}$, the matrix of the residue endomorphism of the logarithmic pole at $z=0$ of the $(T E)$-structure over $t^{0}=0$ is

$$
B^{(1)}(0)=\rho^{(1)} C_{1}+b_{3,0}^{(1)} D .
$$

It is diagonal with the eigenvalues $\rho^{(1)} \pm b_{3,0}^{(1)}$. Therefore the monodromy has the eigenvalues $\exp \left(-2 \pi \mathrm{i}\left(\rho^{(1)} \pm b_{3,0}^{(1)}\right)\right)$.

If $b_{3,0}^{(1)} \in \mathbb{C} \backslash\left(\frac{1}{2} \mathbb{Z} \backslash\{0\}\right)$, the eigenvalues of the residue endomorphism do not differ by a nonzero integer. Because of Theorem 3.23(c), then the monodromy is semisimple.

We will show that the monodromy is also in the cases $b_{3,0}^{(1)} \in \frac{1}{2} \mathbb{Z} \backslash\{0\}$ semisimple, by reducing these cases to the case $b_{3,0}^{(1)}=0$.

Suppose $b_{3,0}^{(1)} \in \frac{1}{2} \mathbb{N}$. The case $b_{3,0}^{(1)} \in \frac{1}{2} \mathbb{Z}_{<0}$ can be reduced to this case by exchanging $v_{1}$ and $v_{2}$. We will construct a new $(T E)$-structure over $\left(M, t^{0}\right)=(\mathbb{C}, 0)$ with the same monodromy and again with trace free pole part and of generic type (Sem) with logarithmic restriction over $t^{0}$, but where $B^{(1)}(0)$ is replaced by

$$
\widetilde{B}^{(1)}(0)=\left(\rho^{(1)}+\frac{1}{2}\right)+\left(b_{3,0}^{(1)}-\frac{1}{2}\right) D .
$$

Applying this sufficiently often, we arrive at the case $b_{3,0}^{(1)}=0$, which has semisimple monodromy. 
The basis $\underline{\widetilde{v}}:=\underline{v} \cdot\left(\begin{array}{cc}1 & 0 \\ 0 & z\end{array}\right)$ of $H^{\prime}:=\left.H\right|_{\mathbb{C}^{*} \times\left(M, t^{0}\right)}$ in a neighborhood of $(0,0)$ defines a new $(T E)$-structure over $(M, 0)$ because of

$$
\begin{aligned}
& z \nabla_{\partial_{t} \underline{\widetilde{v}}}=\underline{\widetilde{v}}\left(z^{-1} a_{2} C_{2}+a_{3} D+z a_{4} E\right) \quad \text { and } \quad a_{2}^{(0)}=0, \\
& z^{2} \nabla_{\partial_{z} \underline{\widetilde{v}}}=\underline{\widetilde{v}}\left(z\left(\rho^{(1)}+\frac{1}{2}\right) C_{1}+z^{-1} b_{2} C_{2}+\left(b_{3}-z \frac{1}{2}\right) D+z b_{4} E\right) \quad \text { and } \quad b_{2}^{(0)}=0 .
\end{aligned}
$$

Of course, it has the same monodromy. The restriction over $t^{0}=0$ has a logarithmic pole at $z=0$ because $b_{2}^{(1)}=\frac{-1}{k_{1}} t a_{2}^{(1)}$ and $b_{3}^{(0)}=\gamma t^{k_{1}}$ with $k_{1} \in \mathbb{N}$. Its generic type is still (Sem). Its numbers $\widetilde{k}_{1}$ and $\widetilde{k}_{2}$ satisfy $\widetilde{k}_{1}+\widetilde{k}_{2}=\operatorname{deg}_{t} \operatorname{det} \widetilde{\mathcal{U}}=\operatorname{deg}_{t}\left(b_{3}^{(0)}\right)^{2}=2 k_{1}$. The assumption $\widetilde{k}_{1}<\widetilde{k}_{2}$ would lead together with part (ii) to two different eigenvalues of the monodromy, a contradiction. Therefore $\widetilde{k}_{1}=\widetilde{k}_{2}=k_{1}$. Thus we are in the same situation as before, with $b_{3,0}^{(1)}$ diminuished by $\frac{1}{2}$.

(b) Suppose that the generic type is (Bra).

$(i)$ and $(i i) \mathcal{U}$ is nilpotent, but not 0 . We can choose a $\mathbb{C}\{t, z\}$-basis $\underline{v}$ of $\mathcal{O}(H)_{(0,0)}$ such that $B^{(0)}=b_{2}^{(0)} C_{2}, \quad$ so $\quad b_{3}^{(0)}=b_{4}^{(0)}=0$.

Then $\delta^{(1)}=-b_{2}^{(0)} b_{4}^{(1)}$. Here $\operatorname{deg}_{t} b_{2}^{(0)}=k_{1}$ and $\operatorname{deg}_{t} \delta^{(1)}=k_{1}+k_{2}$, so $k_{2}=\operatorname{deg}_{t} b_{4}^{(1)} \geq 0$. We can make a coordinate change in $t$ such that afterwards

$$
b_{2}^{(0)} b_{4}^{(1)}=\gamma^{2} t^{k_{1}+k_{2}}
$$

for an arbitrarily chosen $\gamma \in \mathbb{C}^{*}$. Then a diagonal base change leads to a basis which is again called $\underline{v}$ with matrices which are again called $A$ and $B$ with

$$
b_{2}^{(0)}=\gamma t^{k_{1}}, \quad b_{3}^{(0)}=b_{4}^{(0)}=0, \quad b_{4}^{(1)}=\gamma t^{k_{2}} .
$$

The vanishing of the coefficients in $\mathbb{C}\{t\}$ of $C_{2} \cdot z^{0}, D \cdot z^{0}, C_{2} \cdot z^{1}, D \cdot z^{1}$ and $E \cdot z^{2}$ in (6.2)-(6.4) tells the following

$$
\begin{array}{ll}
C_{2} \cdot z^{0}: & a_{3}^{(0)}=0, \\
D \cdot z^{0}: & a_{4}^{(0)}=0, \\
C_{2} \cdot z^{1}: & 0=k_{1} \gamma t^{k_{1}-1}+a_{2}^{(0)}\left(1+2 b_{3}^{(1)}\right)-2 a_{3}^{(1)} \gamma t^{k_{1}}, \\
& \text { so } \quad \operatorname{deg}_{t} a_{2}^{(0)}=k_{1}-1, \quad 0=k_{1} \gamma+a_{2, k_{1}-1}^{(0)}\left(1+2 b_{3,0}^{(1)}\right), \\
D \cdot z^{1}: & a_{2}^{(0)} \gamma t^{k_{2}}=a_{4}^{(1)} \gamma t^{k_{1}}, \quad \text { so } \quad t^{k_{2}}=a_{4}^{(1)} \frac{t^{k_{1}}}{a_{2}^{(0)}}, \\
& \text { so } \quad k_{2}=1+\operatorname{deg} a_{4}^{(1)} \geq 1, \quad \text { and } \quad a_{4}^{(1)}=a_{2}^{(0)} t^{k_{2}-k_{1}}, \\
E \cdot z^{2}: & 0=k_{2} \gamma t^{k_{2}-1}+2 a_{3}^{(1)} \gamma t^{k_{2}}-2 a_{4}^{(1)} b_{3}^{(1)}, \\
& \text { so } 0=k_{2} \gamma-2 a_{2, k_{1}-1}^{(0)} b_{3,0}^{(1)} .
\end{array}
$$

This shows

$$
k_{2} \geq 1, \quad b_{4,0}^{(1)}=0, \quad b_{3,0}^{(1)}=\frac{-k_{2}}{2\left(k_{1}+k_{2}\right)} \in\left(-\frac{1}{2}, 0\right) \cap \mathbb{Q} .
$$

With respect to the basis $\left.\underline{v}\right|_{(0,0)}$ of $K_{(0,0)}$, the matrix of the residue endomorphism of the logarithmic pole at $z=0$ of the (TE)-structure over $t^{0}=0$ is

$$
B^{(1)}(0)=\rho^{(1)} C_{1}+b_{3,0}^{(1)} D+b_{2,0}^{(1)} C_{2} .
$$

It is semisimple with the eigenvalues $\rho^{(1)} \pm b_{3,0}^{(1)}$, whose difference is smaller than 1 . The monodromy is semisimple with the two different eigenvalues $\exp \left(-2 \pi i\left(\rho^{(1)} \pm b_{3,0}^{(1)}\right)\right)$. 


\subsection{1-parameter unfoldings with trace free pole part of logarithmic pure $(T L E)$-structures over a point}

Such unfoldings are themselves pure (TLE)-structures over $(\mathbb{C}, 0)$, see Remark 3.19(iii) respectively [21, Chapter VI, Theorem 2.1] or [7, Theorem 5.1(c)]. Their restrictions over $t^{0}=0$ have a logarithmic pole at $z=0$. Theorem 6.3 classifies such pure $(T L E)$-structures. The underlying $(T E)$-structures were subject of Definition 6.1 and Theorem 6.2. They gave their generic type and invariants $\left(k_{1}, k_{2}\right) \in \mathbb{N}^{2}$ (for the generic types (Sem) and (Bra)) and $k_{1} \in \mathbb{N}$ (for the generic type (Reg)). Theorem 6.3 will give an invariant $k_{1} \in \mathbb{N}$ also for the generic type (Log) with Higgs field $\neq 0$. Lemma $3.9(b)$ gave the invariant $\rho^{(1)} \in \mathbb{C}$. The coordinate on $\mathbb{C}$ is again called $t$.

Theorem 6.3. Any pure rank 2 (TLE)-structure over $\left(M, t^{0}\right)=(\mathbb{C}, 0)$ with trace free pole part and with logarithmic restriction over $t^{0}$ has after a suitable coordinate change in $t$ a unique Birkhoff normal form in the following list. Here the Birkhoff normal form consists of two matrices $A$ and $B$ which are associated to a global basis $\underline{v}$ of $H$ whose restriction to $\{\infty\} \times$ $\left(M, t^{0}\right)$ is flat with respect to the residual connection along $\{\infty\} \times\left(M, t^{0}\right)$, via $z \nabla_{\partial_{t}} \underline{v}=\underline{v} A$ and $z^{2} \nabla_{\partial_{z}} \underline{v}=\underline{v} B$. The matrices have the shape

$$
\begin{aligned}
& A=a_{2}^{(0)} C_{2}+a_{3}^{(0)} D+a_{4}^{(0)} E, \\
& B=z \rho^{(1)} C_{1}-\gamma t A+z b_{2}^{(1)} C_{2}+z b_{3}^{(1)} D,
\end{aligned}
$$

with $a_{2}^{(0)}, a_{3}^{(0)}, a_{4}^{(0)} \in \mathbb{C}[t], \rho^{(1)}, \gamma \in \mathbb{C}, b_{2}^{(1)}, b_{3}^{(1)} \in \mathbb{C}\left(\right.$ so here $z b_{4}^{(1)} E$ does not turn up, resp. $b_{4}^{(1)}$ $=0)$. The left column of the following list gives the generic type of the underlying (TE)-structure and, depending on the type, the invariant $k_{1} \in \mathbb{N}$ or the invariants $k_{1}, k_{2} \in \mathbb{N}$ from Definition 6.1 of the underlying $(T E)$-structure. The invariant $\rho^{(1)} \in \mathbb{C}$ is arbitrary and is not listed in the table. $\zeta \in \mathbb{C}, \alpha_{3} \in \mathbb{R}_{>0} \cup \mathbb{H}, \alpha_{4} \in \mathbb{C} \backslash\{-1\}, k_{1} \in \mathbb{N}$ and $k_{2} \in \mathbb{N}$ are invariants in some cases. In the first 6 cases, $a_{i}^{(0)}$ is determined by $b_{i}^{(0)}=-\gamma t a_{i}^{(0)}$.

\begin{tabular}{c|c|c|c|c|c|c}
\hline $\begin{array}{c}\text { Generic type } \\
\text { and invariants }\end{array}$ & $\gamma$ & $b_{2}^{(0)}$ & $b_{3}^{(0)}$ & $b_{4}^{(0)}$ & $b_{2}^{(1)}$ & $b_{3}^{(1)}$ \\
\hline $\begin{array}{c}\text { Sem }) \\
k_{2}-k_{1}>0 \text { odd } \\
k_{2}-k_{1} \in 2 \mathbb{N}\end{array}$ & $\frac{2}{k_{1}+k_{2}}$ & $t^{k_{1}}$ & 0 & $t^{k_{2}}$ & 0 & $\frac{k_{1}-k_{2}}{2\left(k_{1}+k_{2}\right)}$ \\
$k_{2}=k_{1}$ & $\frac{2}{k_{1}+k_{2}}$ & $t^{k_{1}}$ & $\zeta t^{\left(k_{1}+k_{2}\right) / 2}$ & $\left(1-\zeta^{2}\right) t^{k_{2}}$ & 0 & $\frac{k_{1}-k_{2}}{2\left(k_{1}+k_{2}\right)}$ \\
\hline$($ Bra $), k_{1}, k_{2}$ & $\frac{1}{k_{1}+k_{2}}$ & $t^{k_{1}}$ & $t^{k_{1}+k_{2}}$ & $-t^{k_{1}+2 k_{2}}$ & 0 & $\frac{-k_{2}}{2\left(k_{1}+k_{2}\right)}$ \\
\hline$($ Reg $), k_{1}$ & $\frac{1+\alpha_{4}}{k_{1}}$ & $t^{k_{1}}$ & 0 & 0 & 0 & $\frac{1}{2} \alpha_{4}$ \\
$($ Reg $), k_{1}$ & $\frac{1}{k_{1}}$ & $t^{k_{1}}$ & 0 & 0 & 1 & 0 \\
\hline \hline Generic type & $\gamma$ & $a_{2}^{(0)}$ & $a_{3}^{(0)}$ & $a_{4}^{(0)}$ & $b_{2}^{(1)}$ & $b_{3}^{(1)}$ \\
\hline$($ Log $)$ & 0 & $k_{1} t^{k_{1}-1}$ & 0 & 0 & 0 & $-\frac{1}{2}$ \\
$($ Log $)$ & 0 & 0 & 0 & 0 & 0 & $\alpha_{3}$ \\
$($ Log $)$ & 0 & 0 & 0 & 0 & 1 & 0 \\
\hline
\end{tabular}

Before the proof, several remarks on these Birkhoff normal forms are made. The proof is given after Remark 6.6. 


\section{Remarks 6.4.}

(i) The matrix $B(0)=z B^{(1)}(0)$ is the matrix of the logarithmic pole at $z=0$ of the restriction over $t^{0}=0$ of the $(T E)$-structure. In all cases except the 6 th case and the 9 th case, it is $z\left(\rho^{(1)} C_{1}+b_{3}^{(1)} D\right)$, so it is diagonal. In these cases the monodromy is semisimple with eigenvalues $\exp \left(-2 \pi \mathrm{i}\left(\rho^{(1)} \pm b_{3}^{(1)}\right)\right)$. In the 6 th case and the 9 th case, this matrix is $z\left(\rho^{(1)} C_{1}+C_{2}\right)$. Then the matrix and the monodromy have a $2 \times 2$ Jordan block, and the monodromy has the eigenvalue $\exp \left(-2 \pi \mathrm{i} \rho^{(1)}\right)$. In all cases, the leading exponents (defined in Theorem 4.20) of the logarithmic $(T E)$-structure over $t^{0}$ are called $\alpha_{1}^{0}$ and $\alpha_{2}^{0}$, and they are

$$
\alpha_{1 / 2}^{0}=\rho^{(1)} \pm b_{3}^{(1)}, \quad \text { i.e., } \quad \frac{\alpha_{1}^{0}+\alpha_{2}^{0}}{2}=\rho^{(1)}, \quad \alpha_{1}^{0}-\alpha_{2}^{0}=2 b_{3}^{(1)} .
$$

The 6th and 9th cases turn up again in Theorem 6.7. See Remarks 6.8(iv)-(vi).

(ii) In the generic types (Sem), the critical values satisfy $u_{2}=-u_{1}$ because the pole part is trace free, $-\frac{u_{1}+u_{2}}{2}=\rho^{(0)}=0$. They and the regular singular exponents $\alpha_{1}$ and $\alpha_{2}$ can be calculated with the formulas (4.1) and (4.2):

$$
\begin{aligned}
& \delta^{(0)}=-b_{2}^{(0)} b_{4}^{(0)}-\left(b_{3}^{(0)}\right)^{2}=-t^{k_{1}+k_{2}}, \\
& u_{1 / 2}= \pm \sqrt{\frac{1}{4}\left(u_{1}-u_{2}\right)^{2}}= \pm \sqrt{-\delta^{(0)}}= \pm t^{\left(k_{1}+k_{2}\right) / 2}, \\
& \frac{\alpha_{1}+\alpha_{2}}{2}=\rho^{(1)}, \\
& \alpha_{1}-\alpha_{2}=u_{1}^{-1} \delta^{(1)}= \begin{cases}0, & \text { gen. type (Sem) with } k_{2}-k_{1}>0 \text { odd, } \\
\frac{k_{2}-k_{1}}{k_{1}+k_{2}} \zeta, & \text { gen. type (Sem) with } k_{2}-k_{1} \in 2 \mathbb{N}, \\
-2 \alpha_{3}, & \text { gen. type (Sem) with } k_{2}=k_{1} .\end{cases}
\end{aligned}
$$

If $k_{2}=k_{1}$ then $\left\{\alpha_{1}, \alpha_{2}\right\}=\left\{\alpha_{1}^{0}, \alpha_{2}^{0}\right\}$, but if $k_{2}>k_{1}$ then $\left\{\alpha_{1}, \alpha_{2}\right\} \neq\left\{\alpha_{1}^{0}, \alpha_{2}^{0}\right\}$, except if $\zeta \in\{ \pm 1\}$.

(iii) In the generic type (Bra), $\rho^{(1)} \in \mathbb{C}$ is arbitrary, $b_{3}^{(1)}=\frac{-k_{2}}{2\left(k_{1}+k_{2}\right)}$, and $\delta^{(1)}$ varies as follows,

$$
\delta^{(1)}=\frac{k_{2}}{k_{1}+k_{2}} t^{k_{1}+k_{2}}
$$

(iv) In the 5th, 7th and 8th cases in Theorem 6.3, the monodromy is semisimple and the $(T E)$-structure is regular singular. Associate to it the data in Definition 3.18: $H^{\prime}:=$ $\left.H\right|_{\mathbb{C} \times\left(M, t^{0}\right)}, M^{\text {mon }}, N^{\text {mon }}, \operatorname{Eig}\left(M^{\text {mon }}\right)=\left\{\lambda_{1}, \lambda_{2}\right\}, H^{\infty}, C^{\alpha}$ for $\alpha \in \mathbb{C}$ with $\mathrm{e}^{-2 \pi \mathrm{i} \alpha_{j}} \in$ $\left\{\lambda_{1}, \lambda_{2}\right\}$. The leading exponents of the logarithmic $(T E)$-structure over $t^{0}$ are called $\alpha_{1}^{0}$ and $\alpha_{2}^{0}$ as in $(i)$. The leading exponents of the $(T E)$-structure over $t \in \mathbb{C} \backslash\{0\}$ are now called $\alpha_{1}$ and $\alpha_{2}$. Possibly after renumbering $\lambda_{1}$ and $\lambda_{2}, \alpha_{1}^{0}$ and $\alpha_{2}^{0}$, and $\alpha_{1}$ and $\alpha_{2}$, we have $\mathrm{e}^{-2 \pi \mathrm{i} \alpha_{j}^{0}}=\mathrm{e}^{-2 \pi \mathrm{i} \alpha_{j}}=\lambda_{j}$ and the relations in the following table:

\begin{tabular}{c|c|c|c|c}
\hline In Theorem 6.3 & $\alpha_{1}^{0}$ & $\alpha_{2}^{0}$ & $\alpha_{1}$ & $\alpha_{2}$ \\
\hline 5th case & $\rho^{(1)}+\frac{1}{2} \alpha_{4}$ & $\rho^{(1)}-\frac{1}{2} \alpha_{4}$ & $\alpha_{1}^{0}$ & $\alpha_{2}^{0}-1$ \\
7th case & $\rho^{(1)}-\frac{1}{2}$ & $\rho^{(1)}+\frac{1}{2}$ & $\alpha_{1}^{0}$ & $\alpha_{2}^{0}$ \\
8th case & $\rho^{(1)}+\alpha_{3}$ & $\rho^{(1)}-\alpha_{3}$ & $\alpha_{1}^{0}$ & $\alpha_{2}^{0}$ \\
\hline
\end{tabular}


And there exist sections $s_{j} \in C^{\alpha_{j}} \backslash\{0\}$ with

$$
\begin{aligned}
& \mathcal{O}(H)_{0}=\mathbb{C}\{t, z\}\left(s_{1}+\frac{-1}{1+\alpha_{4}} t^{k_{1}} s_{2}\right) \oplus \mathbb{C}\{t, z\}\left(z s_{2}\right) \quad \text { in the 5th case, } \\
& \mathcal{O}(H)_{0}=\mathbb{C}\{t, z\}\left(s_{1}+t^{k_{1}} z^{-1} s_{2}\right) \oplus \mathbb{C}\{t, z\} s_{2} \quad \text { in the } 7 \text { th case, } \\
& \mathcal{O}(H)_{0}=\mathbb{C}\{t, z\} s_{1} \oplus \mathbb{C}\{t, z\} s_{2} \quad \text { in the } 8 \text { th case. }
\end{aligned}
$$

One confirms (6.14)-(6.16) immediately by calculating the matrices $A$ and $B$ with $z \nabla_{\partial_{t}} \underline{v}=\underline{v} A$ and $z^{2} \nabla_{\partial_{z}} \underline{v}=\underline{v} B$ for $\underline{v}$ the basis in (6.14)-(6.16).

$(v)$ Theorem 6.7 contains for the 6 th and 9th cases in Theorem 6.3 a description similar to part $(i v)$. See Remarks 6.8(iv)-(vi).

Remarks 6.5. These remarks study the behaviour of the (TE)-structures in Theorem 6.3 under pull back via maps $\varphi:(\mathbb{C}, 0) \rightarrow(\mathbb{C}, 0)$. The normal forms in Theorem 6.3 are chosen such that the pull backs by maps $\varphi$ with $\varphi(s)=s^{n}$ for some $n \in \mathbb{N}$ are again normal forms in Theorem 6.3.

(i) A general observation: Let $\left(H \rightarrow \mathbb{C} \times\left(M, t^{0}\right), \nabla\right)$ be a $(T E)$-structure over $\left(M, t^{0}\right)=(\mathbb{C}, 0)$ of rank $r \in \mathbb{N}$. Let $\underline{v}$ be $\mathbb{C}\{t, z\}$-basis of $\mathcal{O}(H)_{0}$ with $z \nabla_{\partial_{t}} \underline{v}=\underline{v} A$ and $z^{2} \nabla_{\partial_{z}} \underline{v}=\underline{v} B$ and $A, B \in M_{r \times r}(\mathbb{C}\{t, z\})$. Choose $n \in \mathbb{N}$ and consider a map $\varphi:(\mathbb{C}, 0) \rightarrow(\mathbb{C}, 0), s \mapsto$ $\varphi(s)=t$. Then the pull back $(T E)$-structure $\varphi^{*}(H, \nabla)$ has the basis $\underline{\widetilde{v}}(z, s)=\underline{v}(z, \varphi(s))$. The matrices $\widetilde{A}, \widetilde{B} \in M_{r \times r}(\mathbb{C}\{s, z\})$ with $z \nabla_{\partial_{s}} \underline{\widetilde{v}}=\underline{\widetilde{v}} \widetilde{A}, z^{2} \nabla_{\partial_{z}} \underline{\widetilde{v}}=\underline{\widetilde{v}} \widetilde{B}$ are

$$
\widetilde{A}=\partial_{s} \varphi(s) \cdot A(z, \varphi(s)), \quad \widetilde{B}=B(z, \varphi(s)) .
$$

(ii) These formulas (6.17) show for the 1st to 7 th cases in the list in Theorem 6.3 the following: The pull back via $\varphi:(\mathbb{C}, 0) \rightarrow(\mathbb{C}, 0)$ with $\varphi(s)=s^{n}$ for some $n \in \mathbb{N}$ of such a $(T E)$ structure with invariants $\left(k_{1}, k_{2}\right)$ or $k_{1}$ is a $(T E)$-structure in the same case, where the invariants $\left(k_{1}, k_{2}\right)$ or $k_{1}$ are replaced by $\left(\widetilde{k}_{1}, \widetilde{k}_{2}\right)=\left(n k_{1}, n k_{2}\right)$ or $\widetilde{k}_{1}=n k_{1}$, and where all other invariants coincide with the old invariants.

(iii) The following table says which of the (TE)-structures in the 1st to 7th cases in the list in Theorem 6.3 are not induced by other such $(T E)$-structures:

\begin{tabular}{l|c}
\hline Generic type and invariants & Not induced if \\
\hline$($ Sem $): k_{2}-k_{1}>0$ odd & $\operatorname{gcd}\left(k_{1}, k_{2}\right)=1$ \\
$($ Sem $): \quad k_{2}-k_{1} \in 2 \mathbb{N}, \zeta=0$ & $\operatorname{gcd}\left(k_{1}, k_{2}\right)=1$ \\
$($ Sem $): \quad k_{2}-k_{1} \in 2 \mathbb{N}, \zeta \neq 0$ & $\operatorname{gcd}\left(k_{1}, \frac{k_{1}+k_{2}}{2}\right)=1$ \\
$($ Sem $): \quad k_{2}=k_{1}$ & $k_{2}=k_{1}=1$ \\
\hline$($ Bra $)$ & $\operatorname{gcd}\left(k_{1}, k_{2}\right)=1$ \\
\hline$($ Reg $): N^{\text {mon }}=0$ & $k_{1}=1$ \\
$($ Reg $): N^{\text {mon }} \neq 0$ & $k_{1}=1$ \\
\hline$($ Log $)$ & $k_{1}=1$ \\
\hline
\end{tabular}

(iv) In the 8th and 9th cases, the $(T E)$-structure is induced by its restriction over $t^{0}$ via the map $\varphi:\left(M, t^{0}\right) \rightarrow\left\{t^{0}\right\}$, so it is constant along $M$.

$(v)$ The formulas (6.14) and (6.15) confirm part (ii) for the 5th and 7th cases in Theorem 6.3. Formula (6.16) confirms part (iv) in the 8th case in Theorem 6.3. Analogous statements to part $(i i)$ and part $(i v)$ hold for the cases in Theorem 6.7. They follow from the formulas (6.26), (6.27) and (6.28) there, which are analogous to (6.14), (6.15) and (6.16). See Remarks 6.8(ii) and (iii). 
Remark 6.6. In the 2 nd and 4 th cases in the list in Theorem 6.3 , another $\mathbb{C}\{t, z\}$-basis $\underline{\widetilde{v}}$ of $\mathcal{O}(H)_{0}$ with nice matrices $\widetilde{A}$ and $\widetilde{B}$ is

$$
\underline{\widetilde{v}}=\underline{v} \cdot T \quad \text { with } \quad T=C_{1}+\frac{a_{3}^{(0)}}{a_{2}^{(0)}} E= \begin{cases}C_{1}+\zeta t^{\left(k_{2}-k_{1}\right) / 2} E & \text { in the 2nd case, } \\ C_{1}+t^{k_{2}} E & \text { in the 4th case. }\end{cases}
$$

In the 2nd case

$$
\begin{aligned}
\widetilde{A} & =-\gamma^{-1}\left(t^{k_{1}-1} C_{2}+t^{k_{2}-1} E\right)+z \frac{k_{2}-k_{1}}{2} \zeta t^{\left(k_{2}-k_{1}-2\right) / 2} E, \\
\widetilde{B} & =z \rho^{(1)} C_{1}-\gamma t \widetilde{A}+z b_{3}^{(1)} D .
\end{aligned}
$$

In the 4 th case

$$
\begin{aligned}
& \widetilde{A}=-\gamma^{-1} t^{k_{1}-1} C_{2}+z k_{2} t^{k_{2}-1} E, \\
& \widetilde{B}=z \rho^{(1)} C_{1}-\gamma t \widetilde{A}+z b_{3}^{(1)} D .
\end{aligned}
$$

These matrices are not in Birkhoff normal form. The basis $\underline{\widetilde{v}}$ is still a global basis of the pure $(T L E)$-structure, but the sections $\left.\widetilde{v}_{j}\right|_{\{\infty\} \times M}$ are not flat with respect to the residual connection along $\{\infty\} \times M$.

Proof of Theorem 6.3. Consider any pure $(T L E)$-structure over $\left(M, t^{0}\right)=(\mathbb{C}, 0)$ with trace free pole part and with logarithmic restriction to $t^{0}$. Choose a global basis $\underline{v}$ of $H$ whose restriction to $\{\infty\} \times\left(M, t^{0}\right)$ is flat with respect to the residual connection along $\{\infty\} \times\left(M, t^{0}\right)$. Its matrices $A$ and $B$ with $z \nabla_{\partial_{t}} \underline{v}=\underline{v} A$ and $z^{2} \nabla_{\partial_{z}} \underline{v}=\underline{v} B$ have because of (3.13) (in Lemma 3.11) the shape (6.7) and

$$
B=z \rho^{(1)} C_{1}+\left(b_{2}^{(0)}+z b_{2}^{(1)}\right) C_{2}+\left(b_{3}^{(0)}+z b_{3}^{(1)}\right) D+\left(b_{4}^{(0)}+z b_{4}^{(1)}\right) E
$$

with $a_{j}^{(0)} \in \mathbb{C}\{t\}, b_{j}^{(0)} \in t \mathbb{C}\{t\}, b_{j}^{(1)} \in \mathbb{C}$. They satisfy the relations (3.19) (and, equivalently, (6.2)-(6.4)), so, more explicitly,

$$
\begin{aligned}
& a_{i}^{(0)} b_{j}^{(0)}=a_{j}^{(0)} b_{i}^{(0)} \quad \text { for } \quad(i, j) \in\{(2,3),(2,4),(3,4)\}, \\
& \left(\begin{array}{l}
-\partial_{t} b_{2}^{(0)} \\
-\partial_{t} b_{3}^{(0)} \\
-\partial_{t} b_{4}^{(0)}
\end{array}\right)=\left(\begin{array}{ccc}
1+2 b_{3}^{(1)} & -2 b_{2}^{(1)} & 0 \\
-b_{4}^{(1)} & 1 & b_{2}^{(1)} \\
0 & 2 b_{4}^{(1)} & 1-2 b_{3}^{(1)}
\end{array}\right)\left(\begin{array}{c}
a_{2}^{(0)} \\
a_{3}^{(0)} \\
a_{4}^{(0)}
\end{array}\right) .
\end{aligned}
$$

First we consider the cases when all $a_{j}^{(0)}$ are 0 . Then also all $b_{j}^{(0)}$ are 0 , because of $b_{j}^{(0)} \in t \mathbb{C}\{t\}$ and because of the differential equations (6.19). Then $B=z B^{(1)}$, and it is clear that this matrix can be brought to the form $B=z \rho^{(1)} C_{1}+z \alpha_{3} D$ or $B=z \rho^{(1)} C_{1}+z C_{2}$ by a constant base change. The number $\alpha_{3} \in \mathbb{C}$ can be replaced by $-\alpha_{3}$, so $\alpha_{3} \in \mathbb{R}_{\geq 0} \cup \mathbb{H}$ is unique. This gives the last two cases in the list. There the generic type is $(\mathrm{Log})$.

For the rest of the proof, we consider the cases when at least one $a_{j}^{(0)}$ is not 0 . Then (6.18) says

$$
\left(b_{2}^{(0)}, b_{3}^{(0)}, b_{4}^{(0)}\right)=\frac{b_{j}^{(0)}}{a_{j}^{(0)}} \cdot\left(a_{2}^{(0)}, a_{3}^{(0)}, a_{4}^{(0)}\right), \quad \text { so } \quad B^{(0)}=\frac{b_{j}^{(0)}}{a_{j}^{(0)}} \cdot A^{(0)} .
$$

If $b_{j}^{(0)}=0$ then $b_{2}^{(0)}=b_{3}^{(0)}=b_{4}^{(0)}=0$, and the generic type is $(\log )$. If $b_{j}^{(0)} \neq 0$, then the generic type is (Sem) or (Bra) or (Reg). Consider for a moment the cases when the residue 
endomorphism of the logarithmic pole at $z=0$ of the $(T E)$-structure over $t^{0}$ is semisimple. By Theorem 6.1, these cases include the generic types (Sem) and (Bra). Then a linear base change gives $b_{2}^{(1)}=b_{4}^{(1)}=0$, so that the $3 \times 3$-matrix in (6.19) is diagonal. Then denote $\widetilde{\beta}_{j}:=\operatorname{deg}_{t} b_{j}^{(0)} \in \mathbb{N}$. A coordinate change in $t$ leads to $b_{j}^{(0)}=b_{j, \widetilde{\beta}_{j}}^{(0)} \cdot t^{\widetilde{\beta}_{j}}$. The differential equation in (6.19) leads to $a_{j}^{(0)}=a_{j, \widetilde{\beta}_{j}-1}^{(0)} \cdot t^{\widetilde{\beta}_{j}-1}$, and to $b_{j}^{(0)} / a_{j}^{(0)}=-\gamma t$ for some $\gamma \in \mathbb{C}^{*}$. Define

$$
\beta_{2}=\frac{1+2 b_{3}^{(1)}}{\gamma}, \quad \beta_{3}=\frac{1}{\gamma}, \quad \beta_{4}=\frac{1-2 b_{3}^{(1)}}{\gamma} .
$$

Now (6.20) and the differential equations in (6.19) show $\widetilde{\beta}_{j}=\beta_{j}$ and

$$
\begin{array}{llll}
b_{2}^{(0)}=0 & \text { or } & & \left(\beta_{2} \in \mathbb{N} \text { and } b_{2}^{(0)}=b_{2, \beta_{2}}^{(0)} \cdot t^{\beta_{2}}\right) \neq 0, \\
b_{3}^{(0)}=0 & \text { or } & \left(\beta_{3} \in \mathbb{N} \text { and } b_{3}^{(0)}=b_{3, \beta_{3}}^{(0)} \cdot t^{\beta_{3}}\right) \neq 0, \\
b_{4}^{(0)}=0 & \text { or } & \left(\beta_{4} \in \mathbb{N} \text { and } b_{4}^{(0)}=b_{4, \beta_{4}}^{(0)} \cdot t^{\beta_{4}}\right) \neq 0 .
\end{array}
$$

Now we discuss the generic types (Sem), (Bra), (Reg) and (Log) separately.

Generic type $(S e m)$. By Theorem 6.2 , we can choose the basis $\underline{v}$ such that $b_{2}^{(1)}=b_{4}^{(1)}=0$. In the cases $k_{2}>k_{1}$, by Theorem $6.2, b_{3}^{(1)}$ is up to the sign unique, and we can choose it to be

$$
b_{3}^{(1)}=\frac{k_{1}-k_{2}}{2\left(k_{1}+k_{2}\right)} \in\left(-\frac{1}{2}, 0\right) \cap \mathbb{Q}
$$

(possibly by exchanging $v_{1}$ and $v_{2}$ ). In the cases $k_{2}=k_{1}$ we write $\alpha_{3}:=b_{3}^{(1)} \in \mathbb{C}$. We can change its sign and get a unique $\alpha_{3} \in \mathbb{R}_{\geq 0} \cup \mathbb{H}$. We make a suitable coordinate change in $t$ and obtain $b_{2}^{(0)}, b_{3}^{(0)}, b_{4}^{(0)}$ as in (6.22). The relations (6.5) and (6.6) still hold. Equation (6.6) implies

$$
\left(b_{2}^{(0)} b_{4}^{(0)} \neq 0, \beta_{2}+\beta_{4}=k_{1}+k_{2}\right) \quad \text { or } \quad\left(b_{3}^{(0)} \neq 0,2 \beta_{3}=k_{1}+k_{2}\right)
$$

(or both). In both cases (6.21) gives

$$
\gamma=\frac{2}{k_{1}+k_{2}} .
$$

Thus

$$
\left(\beta_{2}, \beta_{3}, \beta_{4}\right)=\left\{\begin{array}{lll}
\left(k_{1}, \frac{k_{1}+k_{2}}{2}, k_{2}\right) & \text { if } \quad k_{2}>k_{1}, \\
\left(k_{1}\left(1+2 \alpha_{3}\right), k_{1}, k_{1}\left(1-2 \alpha_{3}\right)\right. & \text { if } \quad k_{2}=k_{1} .
\end{array}\right.
$$

In the cases $k_{2}>k_{1}$, we have $\beta_{2}<\beta_{3}<\beta_{4}$. Then (6.22) and the relation (6.5) imply $b_{2}^{(0)} \neq 0$, so $b_{2, \beta_{2}}^{(0)} \neq 0$. The nonvanishing $\delta^{(0)} \neq 0$ implies $b_{2}^{(0)} b_{4}^{(0)}+\left(b_{3}^{(0)}\right)^{2} \neq 0$.

In the case $k_{2}-k_{1}>0$ even, a linear coordinate change in $t$ and a diagonal base change allow to reduce the triple $\left(b_{2, \beta_{2}}^{(0)}, b_{3, \beta_{3}}^{(0)}, b_{4, \beta_{4}}^{(0)}\right) \in \mathbb{C}^{3}$ to a triple $\left(1, \zeta, 1-\zeta^{2}\right)$ with $\zeta \in \mathbb{C}$ unique.

In the case $k_{2}-k_{1}>0$ odd, we have $\beta_{3} \notin \mathbb{N}$, so $b_{3}^{(0)}=0$, and a linear coordinate change in $t$ and a diagonal base change allow to reduce the pair $\left(b_{2, \beta_{2}}^{(0)}, b_{4, \beta_{4}}^{(0)}\right) \in\left(\mathbb{C}^{*}\right)^{2}$ to the pair $(1,1)$.

In the cases $k_{2}=k_{1}$ and $\alpha_{3} \neq 0,(6.5)$ and (6.23) imply $b_{2}^{(0)}=b_{4}^{(0)}=0$. Then a linear coordinate change in $t$ allows to reduce $b_{3, \beta_{3}}^{(0)}$ to the value 1 .

In the cases $k_{2}=k_{1}$ and $\alpha_{3}=0$, as in the proof of Theorem 6.2(a) (iii), a base change with constant coefficients leads to $b_{2}^{(0)}=b_{4}^{(0)}=0$. Then a linear coordinate change in $t$ allows to 
reduce $b_{3, \beta_{3}}^{(0)}$ to the value 1 . In all cases of generic type (Sem), we obtain the normal forms in the list in Theorem 6.3.

Generic type (Bra). By Theorem 6.2, we can choose the basis $\underline{v}$ such that $b_{2}^{(1)}=b_{4}^{(1)}=0$, and $b_{3}^{(1)}$ is up to the sign unique. We can choose it to be

$$
b_{3}^{(1)}=\frac{-k_{2}}{2\left(k_{1}+k_{2}\right)} \in\left(-\frac{1}{2}, 0\right) \cap \mathbb{Q}
$$

(possibly by exchanging $v_{1}$ and $v_{2}$ ). We make a suitable coordinate change in $t$ and obtain $b_{2}^{(0)}$, $b_{3}^{(0)}, b_{4}^{(0)}$ as in (6.22). The nonvanishing $\delta^{(1)} \neq 0$ and $\operatorname{deg}_{t} \delta^{(1)}=k_{1}+k_{2}$ say

$$
0 \neq \delta^{(1)}=-2 b_{3}^{(1)} b_{3}^{(0)},
$$

so $b_{3}^{(0)} \neq 0$ and

$$
\begin{aligned}
& \frac{1}{\gamma}=\beta_{3}=\operatorname{deg} b_{3}^{(0)}=\operatorname{deg} \delta^{(1)}=k_{1}+k_{2}, \quad \gamma=\frac{1}{k_{1}+k_{2}}, \\
& \left(\beta_{2}, \beta_{3}, \beta_{4}\right)=\left(k_{1}, k_{1}+k_{2}, k_{1}+2 k_{2}\right) .
\end{aligned}
$$

The relation (6.5) still holds, and it implies $b_{2}^{(0)} \neq 0$. The vanishing $\delta^{(0)}=0$ says $b_{2, \beta_{2}}^{(0)} b_{4, \beta_{4}}^{(0)}+$ $\left(b_{3, \beta_{3}}^{(0)}\right)^{2}=0$. Together with $b_{2, \beta_{2}}^{(0)} \neq 0$ and $b_{3, \beta_{3}}^{(0)} \neq 0$ it implies $b_{4, \beta_{4}}^{(0)} \neq 0$. A linear coordinate change in $t$ and a diagonal base change allow to reduce the triple $\left(b_{2, \beta_{2}}^{(0)}, b_{3, \beta_{3}}^{(0)}, b_{4, \beta_{4}}^{(0)}\right) \in\left(\mathbb{C}^{*}\right)^{3}$ to the triple $(1,1,-1)$. We obtain the normal form in the list in Theorem 6.3.

Generic type (Reg). First we consider the case when the residue endomorphism of the logarithmic pole at $z=0$ of the $(T E)$-structure over $t^{0}$ is semisimple. Then a linear base change gives $b_{2}^{(1)}=b_{4}^{(1)}=0$. And a suitable coordinate change in $t$ gives $b_{2}^{(0)}, b_{3}^{(0)}, b_{4}^{(0)}$ as in (6.22).

First consider the case $b_{3}^{(1)} \neq 0$. Then the vanishing $0=\delta^{(1)}=-2 b_{3}^{(1)} b_{3}^{(0)}$ says $b_{3}^{(0)}=0$. Now the vanishing $0=\delta^{(0)}=-b_{2}^{(0)} b_{4}^{(0)}$ says that either $b_{2}^{(0)}=0$ or $b_{4}^{(0)}=0$. Both together cannot be 0 as the generic type is (Reg) and not ( $\log )$. After possibly exchanging $v_{1}$ and $v_{2}$, we suppose $b_{2}^{(0)} \neq 0, b_{4}^{(0)}=0$. Now $k_{1}=\beta_{2}$. Write $\alpha_{4}:=2 b_{3}^{(1)} \in \mathbb{C}$. By $(6.21)$,

$$
\gamma=\frac{1+\alpha_{4}}{k_{1}} \text {. }
$$

A diagonal base change allows to reduce $b_{2, \beta_{2}}^{(0)}$ to 1 .

Now consider the case $b_{3}^{(1)}=0$. Then $\beta_{2}=\beta_{3}=\beta_{4}=1 / \gamma$, and this is equal to $k_{1}$, as $\beta_{j} \in \mathbb{N}$ for at least one $j$. Write $\alpha_{4}:=b_{3}^{(1)}=0$. Then (6.24) still holds. By a base change with constant coefficients, we can obtain $b_{2}^{(0)}=t^{k_{1}}$ and $b_{3}^{(0)}=0$. The vanishing $0=\delta^{(0)}=-b_{2}^{(0)} b_{4}^{(0)}$ tells $b_{4}^{(0)}=0$. For $\alpha_{4} \neq 0$ as well as for $\alpha_{4}=0$, we obtain the normal form in the 5 th case in the list in Theorem 6.3.

Finally consider the case when the residue endomorphism of the logarithmic pole at $z=0$ of the $(T E)$-structure over $t^{0}$ has a $2 \times 2$ Jordan block. A base change with constant coefficients leads to $b_{3}^{(1)}=b_{4}^{(1)}=0$ and $b_{2}^{(1)}=1$. We will lead the assumption $b_{4}^{(0)} \neq 0$ as well as the assumption $b_{4}^{(0)}=0, b_{3}^{(0)} \neq 0$ to a contradiction.

Assume $b_{4}^{(0)} \neq 0$. Denote $\beta_{4}:=\operatorname{deg}_{t} b_{4}^{(0)} \in \mathbb{N}$. A coordinate change in $t$ leads to $b_{4}^{(0)}=\frac{-1}{\beta_{4}} t^{\beta_{4}}$. The differential equation in (6.19) for $b_{4}^{(0)}$ gives $a_{4}^{(0)}=t^{\beta_{4}-1}$. Now (6.20) gives $b_{3}^{(0)}=\frac{-1}{\beta_{4}} t a_{3}^{(0)}$. The differential equation in (6.19) for $b_{3}^{(0)}$ becomes

$$
\partial_{t}\left(t a_{3}^{(0)}\right)=\beta_{4} a_{3}^{(0)}+\beta_{4} t^{\beta_{4}-1} .
$$

This equation has no solution in $\mathbb{C}\{t\}$, a contradiction. 
Assume $b_{4}^{(0)}=0, b_{3}^{(0)} \neq 0$. The same arguments as for the case $b_{4}^{(0)} \neq 0$ give a contradiction if we replace $\left(b_{4}^{(0)}, a_{4}^{(0)}, b_{3}^{(0)}, a_{3}^{(0)}\right)$ by $\left(b_{3}^{(0)}, a_{3}^{(0)}, b_{2}^{(0)}, a_{2}^{(0)}\right)$.

Therefore $b_{4}^{(0)}=0, b_{3}^{(0)}=0, b_{2}^{(0)} \neq 0$. Now $k_{1}=\operatorname{deg}_{t} b_{2}^{(0)}$. A coordinate change in $t$ leads to $b_{2}^{(0)}=t^{k_{1}}$. The differential equations (6.19) gives $a_{4}^{(0)}=a_{3}^{(0)}=0, a_{2}^{(0)}=-k_{1} t^{k_{1}-1}$. We obtain the normal form in the 6th case in the list in Theorem 6.3.

Generic type $(\log )$. Now $b_{2}^{(0)}=b_{3}^{(0)}=b_{4}^{(0)}=0$. The cases when all $a_{i}^{(0)}=0$, were considered above. We assume now $a_{j}^{(0)} \neq 0$ for some $j \in\{2,3,4\}$. The equations (6.19) become a homogeneous system of linear equations with a nontrivial solution. Therefore the determinant of the $3 \times 3$-matrix in $(6.19)$ vanishes. It is $1-4\left(b_{3}^{(1)}\right)^{2}-4 b_{2}^{(1)} b_{4}^{(1)}$. Its vanishing tells $\operatorname{det}\left(B^{(1)}-\right.$ $\left.z \rho^{(1)} C_{1}\right)=\frac{-1}{4}$. As $\operatorname{tr}\left(B^{(1)}-z \rho^{(1)} C_{1}\right)=0$, this matrix has the eigenvalues $\pm \frac{1}{2}$. Therefore a linear base change gives

$$
b_{2}^{(1)}=b_{4}^{(1)}=0, \quad b_{3}^{(1)}=-\frac{1}{2} .
$$

Now the system of equations (6.19) gives $a_{3}^{(0)}=a_{4}^{(0)}=0$, whereas $a_{2}^{(0)}$ is arbitrary in $\mathbb{C}\{t\} \backslash\{0\}$. Denote $k_{1}:=1+\operatorname{deg}_{t} a_{2}^{(0)} \in \mathbb{N}$. A coordinate change in $t$ leads to $a_{2}^{(0)}=k_{1} t^{k_{1}-1}$. We obtain the normal form in the seventh case in the list in Theorem 6.3.

\subsection{Generically regular singular $(T E)$-structures over $(\mathbb{C}, 0)$ with logarithmic restriction over $t^{0}=0$ and not semisimple monodromy}

The only 1-parameter unfoldings with trace free pole part of logarithmic $(T E)$-structures over a point, which are not covered by Theorem 6.3, have generic type (Reg) or (Log) and not semisimple monodromy. This follows from Theorem 6.2 and Theorem 3.20(a). These (TE)structures are classified in Theorem 6.7. Some of them are in the 6th or 9th case in Theorem 6.3, but most are not.

Theorem 6.7. Consider a rank $2(T E)$-structure $\left(H \rightarrow \mathbb{C} \times\left(M, t^{0}\right), \nabla\right)$ over $\left(M, t^{0}\right)=(\mathbb{C}, 0)$ which is generically regular singular (so of generic type (Reg) or (Log)), which has trace free pole part, whose restriction over $t^{0}$ is logarithmic, and whose monodromy has a $2 \times 2$ Jordan block. Associate to it the data in Definition 3.18: $H^{\prime}:=\left.H\right|_{\mathbb{C} \times\left(M, t^{0}\right)}, M^{\mathrm{mon}}, N^{\mathrm{mon}}, \operatorname{Eig}\left(M^{\mathrm{mon}}\right)=\{\lambda\}$, $H^{\infty}, C^{\alpha}$ for $\alpha \in \mathbb{C}$ with $\mathrm{e}^{-2 \pi \mathrm{i} \alpha}=\lambda$.

The leading exponents of the $(T E)$-structures over $t \neq 0$ come from Theorem $4.5(b)$ if the generic type is (Reg) and from Theorem 4.20(b) if the generic type is (Log). In both cases the leading exponents are independent of $t$ and are still called $\alpha_{1}$ and $\alpha_{2}$. Recall $\alpha_{1}-\alpha_{2} \in \mathbb{N}_{0}$. The leading exponents of the logarithmic (TE)-structure over $t^{0}=0$ from Theorem $4.20(b)$ are now called $\alpha_{1}^{0}$ and $\alpha_{2}^{0}$. Recall $\alpha_{1}^{0}-\alpha_{2}^{0} \in \mathbb{N}_{0}$.

Precisely one of the three cases (I), (II) and (III) in the following table holds:

\begin{tabular}{l|l|l|l}
\hline case $(I)$ & $\alpha_{1}^{0}=\alpha_{1}$ & $\alpha_{2}^{0}=\alpha_{2}+1$ & thus $\alpha_{1}-\alpha_{2} \in \mathbb{N}$ \\
case $(I I)$ & $\alpha_{1}^{0}=\alpha_{1}+1$ & $\alpha_{2}^{0}=\alpha_{2}$ & \\
case $(I I I)$ & $\alpha_{1}^{0}=\alpha_{1}$ & $\alpha_{2}^{0}=\alpha_{2}$ & \\
\hline
\end{tabular}

Choose any section $s_{1} \in C^{\alpha_{1}} \backslash \operatorname{ker}\left(\nabla_{z \partial_{z}}-\alpha_{1}: C^{\alpha_{1}} \rightarrow C^{\alpha_{1}}\right)$. It determines uniquely a section $s_{2} \in \operatorname{ker}\left(\nabla_{z \partial_{z}}-\alpha_{2}: C^{\alpha_{2}} \rightarrow C^{\alpha_{2}}\right) \backslash\{0\}$ with

$$
\left(\nabla_{z \partial_{z}}-\alpha_{1}\right)\left(s_{1}\right)=z^{\alpha_{1}-\alpha_{2}} s_{2}
$$


Then

$$
\begin{aligned}
& \mathcal{O}(H)_{(0,0)}=\mathbb{C}\{t, z\}\left(s_{1}+f s_{2}\right) \oplus \mathbb{C}\{t, z\} z s_{2} \text { for some } f \in t \mathbb{C}\{t\} \backslash\{0\} \text { in case }(I), \\
& \mathcal{O}(H)_{(0,0)}=\mathbb{C}\{t, z\}\left(s_{2}+f s_{1}\right) \oplus \mathbb{C}\{t, z\} z s_{1} \text { for some } f \in t \mathbb{C}\{t\} \backslash\{0\} \text { in case }(I I), \\
& \mathcal{O}(H)_{(0,0)}=\mathbb{C}\{t, z\} s_{1} \oplus \mathbb{C}\{t, z\} s_{2} \quad \text { in case }(I I I)
\end{aligned}
$$

The function $f$ in the cases (6.26) and (6.27) is independent of the choice of $s_{1}$, so it is an invariant of the gauge equivalence class of the (TE)-structure.

Before the proof, some remarks are made.

\section{Remarks 6.8.}

(i) Equation (6.25) gives

$$
\nabla_{z \partial_{z}}\left(\left(s_{1}, s_{2}\right)\right)=\left(s_{1}, s_{2}\right)\left(\begin{array}{cc}
\alpha_{1} & 0 \\
z^{\alpha_{1}-\alpha_{2}} & \alpha_{2}
\end{array}\right)=\left(s_{1}, s_{2}\right) \cdot z^{-1} B
$$

with

$$
B=z \frac{\alpha_{1}+\alpha_{2}}{2} C_{1}+z^{\alpha_{1}-\alpha_{2}+1} C_{2}+z \frac{\alpha_{1}-\alpha_{2}}{2} D
$$

(ii) The generic type is (Log) in the case (III). This (TE)-structure is induced by its restriction over $t^{0}=0$ via the projection $\varphi:\left(M, t^{0}\right) \rightarrow\left\{t^{0}\right\}$. The matrices $A$ and $B$ for the basis $\underline{v}=\left(s_{1}, s_{2}\right)$ are $A=0$ and $B$ as in (6.29).

(iii) The generic type is (Reg) in the cases (I) and (II). In these cases the (TE)-structure is induced by the special cases of (6.26) respectively (6.27) with $\widetilde{f}=t$ via the map $\varphi=f:(\mathbb{C}, 0) \rightarrow(\mathbb{C}, 0)$.

(iv) The matrices $A$ and $B$ for the basis $\underline{v}=\left(s_{1}+f s_{2}, z s_{2}\right)$ in $(6.26)\left(\Rightarrow\right.$ case $\left.(\mathrm{I}), \Rightarrow \alpha_{1}-\alpha_{2} \in \mathbb{N}\right)$ are

$$
A=\partial_{t} f \cdot C_{2}, \quad B=\left(\begin{array}{cc}
z \alpha_{1} & 0 \\
\left(\alpha_{2}-\alpha_{1}\right) f+z^{\alpha_{1}-\alpha_{2}} & z\left(\alpha_{2}+1\right)
\end{array}\right) .
$$

The matrices $A$ and $B$ for the basis $\underline{v}=\left(s_{2}+f s_{1}, z s_{1}\right)$ in $(6.27)\left(\Rightarrow\right.$ case (II), $\Rightarrow \alpha_{1}-\alpha_{2}$ $\left.\in \mathbb{N}_{0}\right)$ are

$$
A=\partial_{t} f \cdot C_{2}, \quad B=\left(\begin{array}{cc}
z \alpha_{2}+z^{\alpha_{1}-\alpha_{2}+1} f & z^{\alpha_{1}-\alpha_{2}+2} \\
\left(\alpha_{1}-\alpha_{2}\right) f-z^{\alpha_{1}-\alpha_{2}} f^{2} & z\left(\alpha_{1}+1\right)-z^{\alpha_{1}-\alpha_{2}+1} f
\end{array}\right) .
$$

$(v)$ The invariant $k_{1} \in \mathbb{N}$ from (6.1) is here $k_{1}=\operatorname{deg}_{t} f \in \mathbb{N}$ in the case (6.26) and the case $\left((6.27)\right.$ and $\left.\alpha_{1}-\alpha_{2} \in \mathbb{N}\right)$. It is $k_{1}=2 \operatorname{deg}_{t} f \in 2 \mathbb{N}$ in the case $\left((6.27)\right.$ and $\left.\alpha_{1}=\alpha_{2}\right)$. A suitable coordinate change in $t$ reduces $f$ to $f=t^{k_{1}}$ respectively $f=t^{k_{1} / 2}$.

(vi) The overlap of the (TE)-structures in Theorem 6.3 and in Theorem 6.7 is as follows.

The case (6.26) with $\alpha_{1}=\alpha_{2}+1$ and $f=-t^{k_{1}}$ is the 6th case in Theorem 6.3 with $\rho^{(1)}=\alpha_{1}$.

The case (6.28) with $\alpha_{1}=\alpha_{2}$ is the 9 th case in Theorem 6.3 with $\rho^{(1)}=\alpha_{1}$.

Proof of Theorem 6.7. Choose any section $s_{1}^{0} \in C^{\alpha_{1}^{0}} \backslash \operatorname{ker}\left(\nabla_{z \partial_{z}}-\alpha_{1}^{0}: C^{\alpha_{1}^{0}} \rightarrow C^{\alpha_{1}^{0}}\right) \backslash\{0\}$. It determines uniquely a section $s_{2}^{0} \in \operatorname{ker}\left(\nabla_{z \partial_{z}}: C^{\alpha_{2}^{0}} \rightarrow C^{\alpha_{2}^{0}}\right) \backslash\{0\}$ with

$$
\left(\nabla_{z \partial_{z}}-\alpha_{1}^{0}\right) s_{1}^{0}=z^{\alpha_{1}^{0}-\alpha_{2}^{0}} s_{2}^{0}
$$


Then

$$
\mathcal{O}\left(\left.H\right|_{\mathbb{C} \times\left\{t^{0}\right\}}\right)_{0}=\left.\left.\mathbb{C}\{z\} s_{1}^{0}\right|_{\mathbb{C} \times\left\{t^{0}\right\}} \oplus \mathbb{C}\{z\} s_{2}^{0}\right|_{\mathbb{C} \times\left\{t^{0}\right\}} .
$$

Choose a $\mathbb{C}\{t, z\}$-basis $\underline{v}=\left(v_{1}, v_{2}\right)$ of $\mathcal{O}(H)_{(0,0)}$ which extends this $\mathbb{C}\{z\}$-basis of $\mathcal{O}\left(\left.H\right|_{\mathbb{C} \times\left\{t^{0}\right\}}\right)_{0}$. It has the shape

$$
\begin{array}{ll}
\underline{v}=\left(s_{1}^{0}, s_{2}^{0}\right) \cdot F \quad \text { with } & F=\left(\begin{array}{ll}
f_{1} & f_{2} \\
f_{3} & f_{4}
\end{array}\right) \quad \text { and } \\
f_{1}, f_{4} \in \mathbb{C}\{t, z\}\left[z^{-1}\right], \quad & f_{1}(z, 0)=f_{4}(z, 0)=1, \quad f_{2}, f_{3} \in t \mathbb{C}\{t, z\}\left[z^{-1}\right] .
\end{array}
$$

We write $f_{j}=\sum_{k \geq \operatorname{deg}_{z} f_{j}} f_{j}^{(k)} z^{k}$ with $f_{j}^{(k)}=\sum_{l \geq 0} f_{j, l}^{(k)} t^{l} \in \mathbb{C}\{t\}$ and $f_{j, l}^{(k)} \in \mathbb{C}$. Also we write $\operatorname{det} F=\sum_{k \geq \operatorname{deg}_{z} \operatorname{det} F}(\operatorname{det} F)^{(k)} z^{k}$ with $(\operatorname{det} F)^{(k)} \in \mathbb{C}\{t\}$.

A meromorphic function $g \in \mathbb{C}\{t, z\}\left[z^{-1}\right]$ on a neighborhood $U \subset \mathbb{C} \times M$ which is holomorphic and not vanishing on $(U \backslash\{0\} \times M) \cup\{(0,0)\}$ is in $\mathbb{C}\{t, z\}^{*}$. This and the facts that $\underline{v}$ and $\left(s_{1}^{0}, s_{2}^{0}\right)$ are bases of $\left.H\right|_{U \backslash\{0\} \times M}$ for some neighborhood $U \subset \mathbb{C} \times M$ of $(0,0)$ and that $\left.\underline{v}\right|_{\mathbb{C} \times\left\{t^{0}\right\}}=\left(s_{1}^{0}, s_{2}^{0}\right)$ imply

$$
(\operatorname{det} F) \in \mathbb{C}\{t, z\}^{*}, \quad \text { so especially } \quad(\operatorname{det} F)^{(k)}=0 \quad \text { for } \quad k<0 .
$$

Write $k_{j}:=\operatorname{deg}_{z} f_{j} \in \mathbb{Z} \cup\{\infty\}$ ( $\infty$ if $f_{j}=0$ ). Recall (6.32). It implies $f_{1}^{(0)}, f_{4}^{(0)} \in \mathbb{C}\{t\}^{*}$ and $f_{1}^{(k)}, f_{4}^{(k)} \in t \mathbb{C}\{t\}$ for $k \neq 0$ and $f_{2}^{(k)}, f_{3}^{(k)} \in t \mathbb{C}\{t\}$ for all $k$. Especially $k_{1} \leq 0$ and $k_{4} \leq 0$. We distinguish four cases. Precisely one of them holds:

$$
\begin{array}{lll}
\text { Case } \widetilde{(\mathrm{I})}: & 0=k_{1} \leq k_{2}, & 0>\min \left(k_{3}, k_{4}\right), \\
\text { Case } \widetilde{(\mathrm{II}):} & 0=k_{4} \leq k_{3}, & 0>\min \left(k_{1}, k_{2}\right), \\
\text { Case } \widetilde{(\mathrm{III}):} & 0=k_{1}=k_{4}, & 0 \leq k_{2}, \quad 0 \leq k_{3}, \\
\text { Case } \widetilde{(\mathrm{IV}):} & 0>\min \left(k_{1}, k_{2}\right), & 0>\min \left(k_{3}, k_{4}\right) .
\end{array}
$$

We will show: Case $\widetilde{(\mathrm{I})}$ leads to $(6.26)$ and case (I), case $\widetilde{(\mathrm{II})}$ leads to (6.27) and case (II), case (III) leads to $(6.28)$ and case (III), and case (IV) is impossible.

Case $\widetilde{(I I I)}$ : Then $F \in \mathrm{GL}_{2}(\mathbb{C}\{t, z\})$ and a base change leads to the new basis $\underline{\widetilde{v}}=\left(s_{1}^{0}, s_{2}^{0}\right)$. With

$$
\left(\alpha_{1}, \alpha_{2}, s_{1}, s_{2}\right)=\left(\alpha_{1}^{0}, \alpha_{2}^{0}, s_{1}^{0}, s_{2}^{0}\right)
$$

this gives (6.28) and case (III).

Case $\widetilde{(I)}$ : Then $f_{1} \in \mathbb{C}\{t, z\}^{*}$, and a base change leads to a new basis $\underline{v}^{[1]}=\left(s_{1}^{0}, s_{2}^{0}\right) \cdot F^{[1]}$ with

$$
f_{1}^{[1]}=1, \quad f_{2}^{[1]}=0, \quad f_{4}^{[1]}=\operatorname{det} F^{[1]} \in \mathbb{C}\{t, z\}^{*}, \quad f_{3}^{[1]} \in t \mathbb{C}\{t, z\}\left[z^{-1}\right] .
$$

As $k_{4}^{[1]}=0$, we have $k_{3}^{[1]}<0$. A base change leads to a new basis $\underline{v}^{[2]}=\left(s_{1}^{0}, s_{2}^{0}\right) \cdot F^{[2]}$ with

$$
F^{[2]}=C_{1}+f_{3}^{[2]} C_{2}, \quad \text { with } \quad f_{3}^{[2]} \in t z^{-1} \mathbb{C}\{t\}\left[z^{-1}\right] \backslash\{0\} .
$$

The covariant derivative $z \nabla_{\partial_{t}} v_{1}^{[2]}=z \partial_{t} f_{3}^{[2]} \cdot v_{2}^{[2]}$ must be in $\mathcal{O}(H)_{0}$. This shows $f_{3}^{[2]} \in t z^{-1} \mathbb{C}\{t\}$ $\backslash\{0\}$. With

$$
\left(\alpha_{1}, \alpha_{2}, s_{1}, s_{2}, f\right)=\left(\alpha_{1}^{0}, \alpha_{2}^{0}-1, s_{1}^{0}, z^{-1} s_{2}^{0}, z f_{3}^{[2]}\right),
$$

this gives (6.26) and case (II). 
Case $\widetilde{(I I)}$ : Then $f_{4} \in \mathbb{C}\{t, z\}^{*}$, and a base change leads to a new basis $\underline{v}^{[1]}=\left(s_{1}^{0}, s_{2}^{0}\right) \cdot F^{[1]}$ with

$$
f_{4}^{[1]}=1, \quad f_{3}^{[1]}=0, \quad f_{1}^{[1]}=\operatorname{det} F^{[1]} \in \mathbb{C}\{t, z\}^{*}, \quad f_{2}^{[1]} \in t \mathbb{C}\{t, z\}\left[z^{-1}\right] .
$$

As $k_{1}^{[1]}=0$, we have $k_{2}^{[1]}<0$. A base change leads to a new basis $\underline{v}^{[2]}=\left(s_{1}^{0}, s_{2}^{0}\right) \cdot F^{[2]}$ with

$$
F^{[2]}=C_{1}+f_{2}^{[2]} E, \quad \text { with } \quad f_{2}^{[2]} \in t z^{-1} \mathbb{C}\{t\}\left[z^{-1}\right] \backslash\{0\} .
$$

The covariant derivative $z \nabla_{\partial_{t}} v_{2}^{[2]}=z \partial_{t} f_{2}^{[2]} \cdot v_{1}^{[2]}$ must be in $\mathcal{O}(H)_{0}$. This shows $f_{2}^{[2]} \in t z^{-1} \mathbb{C}\{t\}$ $\backslash\{0\}$. With

$$
\left(\alpha_{1}, \alpha_{2}, s_{1}, s_{2}\right)=\left(\alpha_{1}^{0}-1, \alpha_{2}^{0}, z^{-1} s_{1}^{0}, s_{2}^{0}\right),
$$

this gives (6.27) and almost case (II). "Almost" because we still have to show $\alpha_{1}-\alpha_{2} \in \mathbb{N}_{0}$. This follows from the summand $-z^{\alpha_{1}-\alpha_{2}} f^{2}$ in the left lower entry in the matrix $B$ in (6.31).

Case $\widetilde{(I V)}$ : Exchange $v_{1}$ and $v_{2}$ if $k_{1}>k_{2}$ or if $k_{1}=k_{2}$ and $\operatorname{deg}_{t} f_{1}^{\left(k_{1}\right)}>\operatorname{deg}_{t} f_{2}^{\left(k_{1}\right)}$. Keep the basis $\underline{v}$ if not. The new basis $\underline{v}^{[1]}$ satisfies $\min \left(k_{1}, k_{2}\right)=k_{1}^{[1]} \leq k_{2}^{[1]}$, and in the case $k_{1}^{[1]}=k_{2}^{[1]}$ it satisfies $\operatorname{deg}_{t}\left(f_{1}^{[1]}\right)^{\left(k_{1}^{[1]}\right)} \leq \operatorname{deg}_{t}\left(f_{2}^{[1]}\right)^{\left(k_{1}^{[1]}\right)}$.

By replacing $v_{2}^{[1]}$ by a suitable element in $v_{2}^{[1]}+\mathbb{C}\{t, z\} v_{1}^{[1]}$, we obtain a new basis $\underline{v}^{[2]}$ either with $f_{2}^{[2]}=0$ or with $k_{1}^{[2]}<k_{2}^{[2]}$ and $\operatorname{deg}_{t}\left(f_{1}^{[2]}\right)^{\left(k_{1}^{[2]}\right)}>\operatorname{deg}_{t}\left(f_{2}^{[2]}\right)^{\left(k_{2}^{[2]}\right)}$.

The case $f_{2}^{[2]}=0$ is impossible, as then we would have $f_{1}^{[2]} f_{4}^{[2]}=\operatorname{det} F^{[2]} \in \mathbb{C}\{t, z\}^{*}$, so $f_{1}^{[2]} \in \mathbb{C}\{t, z\}^{*}$ and $0=k_{1}^{[2]}$, but also $k_{1}^{[2]}=k_{1}^{[1]}=\min \left(k_{1}, k_{2}\right)<0$. For the same reason, $f_{3}^{[2]}=0$ is impossible.

$f_{4}^{[2]}=0$ is impossible as then we would have $-f_{2}^{[2]} f_{3}^{[2]}=\operatorname{det} F^{[2]} \in \mathbb{C}\{t, z\}^{*}$, so $f_{2}^{[2]}, f_{3}^{[2]} \in$ $\mathbb{C}\{t, z\}^{*}, 0=k_{2}^{[2]}=k_{3}^{[2]}$ and $k_{4}^{[2]}=\infty$, so $0=\min \left(k_{3}^{[2]}, k_{4}^{[2]}\right)=\min \left(k_{3}, k_{4}\right)<0$, a contradiction.

Write

$$
\begin{array}{ll}
l_{2}:=\operatorname{deg}_{t}\left(f_{2}^{[2]}\right)^{\left(k_{2}^{[2]}\right)} \in \mathbb{N}_{0}, & l_{1}:=\operatorname{deg}_{t}\left(f_{1}^{[2]}\right)^{\left(k_{1}^{[2]}\right)}-l_{2} \in \mathbb{N}, \\
l_{3}:=\operatorname{deg}_{t}\left(f_{3}^{[2]}\right)^{\left(k_{3}^{[2]}\right)} \in \mathbb{N}_{0}, & l_{4}:=\operatorname{deg}_{t}\left(f_{4}^{[2]}\right)^{\left(k_{4}^{[2]}\right)} \in \mathbb{N}_{0} .
\end{array}
$$

Multiplying $v_{1}^{[2]}$ and $v_{2}^{[2]}$ by suitable units in $\mathbb{C}\{t\}$, we obtain a basis $\underline{v}^{[3]}$ with $k_{j}^{[3]}=k_{j}^{[2]}$ and

$$
\begin{array}{ll}
\left(f_{1}^{[3]}\right)^{\left(k_{1}^{[3]}\right)}=t^{l_{1}+l_{2}}, & \left(f_{2}^{[3]}\right)^{\left(k_{2}^{[3]}\right)}=t^{l_{2}}, \\
\left(f_{3}^{[3]}\right)^{\left(k_{3}^{[3]}\right)}=t^{l_{3}} \cdot u_{3}, & \left(f_{4}^{[3]}\right)^{\left(k_{4}^{[3]}\right)}=t^{l_{4}} \cdot u_{4}
\end{array}
$$

for some units $u_{3}, u_{4} \in \mathbb{C}\{t\}^{*}$. We still have $0>k_{1}^{[3]}<k_{2}^{[3]}$ and $\min \left(k_{3}^{[3]}, k_{4}^{[3]}\right)<0$. Consider

$$
z \nabla_{\partial_{t}}\left(v_{1}^{[3]}\right)=z \partial_{t} f_{1}^{[3]} \cdot s_{1}^{0}+z \partial_{t} f_{3}^{[3]} \cdot s_{2}^{0} \in \mathcal{O}(H)_{(0,0)}=\mathbb{C}\{t, z\} v_{1}^{[3]} \oplus \mathbb{C}\{t, z\} v_{2}^{[3]} .
$$

The leading nonvanishing monomial in $z \partial_{t} f_{1}^{[3]}$ is $z^{k_{1}^{[3]}+1} t^{l_{1}+l_{2}-1}$. This implies $k_{2}^{[3]}=k_{1}^{[3]}+1 \leq 0$. Therefore $k_{1}^{[3]}+k_{4}^{[3]}<0$ or $k_{2}^{[3]}+k_{3}^{[3]}<0$. Each part $\left(\operatorname{det} F^{[3]}\right)^{(k)}$ for $k<0$ vanishes. This shows

$$
\begin{aligned}
& k_{1}^{[3]}+k_{4}^{[3]}=k_{2}^{[3]}+k_{3}^{[3]}<0, \quad \text { so } \quad k_{4}^{[3]}=k_{3}^{[3]}+1 \leq 0, \quad k_{3}^{[3]}<0, \\
& 0=\left(f_{1}^{[3]}\right)^{\left(k_{1}^{[3]}\right)}\left(f_{4}^{[3]}\right)^{\left(k_{3}^{[3]}+1\right)}-\left(f_{2}^{[3]}\right)^{\left(k_{1}^{[3]}+1\right)}\left(f_{3}^{[3]}\right)^{\left(k_{3}^{[3]}\right)}=t^{l_{2}}\left(t^{l_{1}+l_{4}} u_{4}-t^{l_{3}} u_{3}\right), \\
& \text { so } \quad l_{3}=l_{1}+l_{4}, \quad u_{3}=u_{4} .
\end{aligned}
$$


We can write

$$
\underline{v}^{[3]}=\left(s_{1}^{0}, s_{2}^{0}\right)\left(\begin{array}{cc}
\left(t^{l_{1}+l_{2}}+z g_{1}\right) z^{k_{1}^{[3]}} & \left(t^{l_{2}}+z g_{2}\right) z^{k_{1}^{[3]}+1} \\
\left(t^{l_{1}+l_{4}} u_{3}+z g_{3}\right) z^{k_{3}^{[3]}} & \left(t^{l_{4}} u_{3}+z g_{4}\right) z^{k_{3}^{[3]}+1}
\end{array}\right)
$$

with some suitable $g_{1}, g_{2}, g_{3}, g_{4} \in \mathbb{C}\{t, z\}$. This shows

$$
\begin{aligned}
\mathcal{O}(H)_{(0,0)} & \cap\left(z^{k_{1}^{[3]}+2} \mathbb{C}\{t, z\} s_{1}^{0}+\mathbb{C}\{t, z\}\left[z^{-1}\right] s_{2}^{0}\right) \\
= & z^{2} \mathbb{C}\{t, z\} v_{1}^{[3]}+z \mathbb{C}\{t, z\} v_{2}^{[3]}+\mathbb{C}\{t, z\}\left(z v_{1}^{[3]}-t^{l_{1}} v_{2}^{[3]}\right) \\
& \subset \mathcal{O}(H)_{(0,0)} \cap\left(\mathbb{C}\{t, z\}\left[z^{-1}\right] s_{1}^{0}+z^{k_{3}^{[3]}+2} \mathbb{C}\{t, z\} s_{2}^{0}\right) .
\end{aligned}
$$

Now consider the element

$$
\begin{aligned}
& z\left(\nabla_{z \partial_{z}}-\left(\alpha_{1}^{0}+k_{1}^{[3]}\right)\right)\left(v_{1}^{[3]}\right) \\
& =z^{2} \partial_{z}\left(z g_{1}\right) z^{k_{1}^{[3]}} s_{1}^{0}+\left(t^{l_{1}+l_{2}}+z g_{1}\right) z^{k_{1}^{[3]}+1+\alpha_{1}^{0}-\alpha_{2}^{0}} s_{2}^{0}+z^{2} \partial_{z}\left(z g_{3}\right) z^{k_{3}^{[3]}} s_{2}^{0} \\
& \quad+\left(t^{l_{1}+l_{4}} u_{3}+z g_{3}\right)\left(k_{3}^{[3]}+\alpha_{2}^{0}-\alpha_{1}^{0}-k_{1}^{[3]}\right) z^{k_{3}^{[3]}+1} s_{2}^{0}
\end{aligned}
$$

of $\mathcal{O}(H)_{(0,0)}$. It is contained in the first line of (6.33), and therefore also in the third line of (6.33). But this leads to a contradiction, when we compare the coefficient of $s_{2}^{0}$. Here observe

$$
k_{3}^{[3]}+\alpha_{2}^{0}-\alpha_{1}^{0}-k_{1}^{[3]}\left\{\begin{array}{l}
< \\
= \\
>
\end{array}\right\} 0 \Longleftrightarrow k_{1}^{[3]}+1+\alpha_{1}^{0}-\alpha_{2}^{0}\left\{\begin{array}{l}
< \\
= \\
>
\end{array}\right\} k_{3}^{[3]}+1 .
$$

This contradiction shows that case $\widetilde{(\mathrm{IV})}$ is impossible.

\section{$7 \quad$ Marked regular singular rank $2(T E)$-structures}

The regular singular rank $2(T E)$-structures over points were subject of Sections 4.5 and 4.6, those over $(\mathbb{C}, 0)$ were subject of Theorem 6.3 and Remark 6.4(iv) and of Theorem 6.7 and Remarks 6.8 .

First we will consider in Remarks 7.1(i)+(ii) regular singular rank $2(T E)$-structures over $\mathbb{P}^{1}$, which arise naturally from Theorems 4.17 and 4.20 . The $(T E)$-structures over the germs $\left(\mathbb{P}^{1}, 0\right)$ and $\left(\mathbb{P}^{1}, \infty\right)$ appeared already in Remark $6.4(i v)$ and in Theorem 6.7.

With the construction in Lemma $3.10(d)$, each of these $(T E)$-structures over $\mathbb{P}^{1}$ extends to a rank $2(T E)$-structure of generic type $(\mathrm{Reg})$ or $(\mathrm{Log})$ over $\mathbb{C} \times \mathbb{P}^{1}$ with primitive Higgs field. With Theorem 3.14, the base manifold $\mathbb{C} \times \mathbb{P}^{1}$ obtains a canonical structure as $F$-manifold with Euler field. For each $t^{0} \in \mathbb{C} \times \mathbb{C}^{*}$, the $(T E)$-structure over the germ $\left(\mathbb{C} \times \mathbb{P}^{1}, t^{0}\right)$ is a universal unfolding of its restriction over $t^{0}$. For each $t^{0} \in \mathbb{C} \times\{0, \infty\}$, the $(T E)$-structure over the germ $\left(\mathbb{C} \times \mathbb{P}^{1}, t^{0}\right)$ will reappear in Theorems 8.1, 8.5 and 8.6. See Remarks 7.2 $(i)+(i i)$.

Then we will observe in Corollary 7.3 that any marked regular singular $(T E)$-structure is a good family of marked regular singular $(T E)$-structures (over points) in the sense of Definition $3.26(b)$.

In Theorem 7.4 we will determine the moduli spaces $M^{\left(H^{\mathrm{ref}, \infty}, M^{\mathrm{ref}}\right) \text {,reg }}$ for marked regular singular rank $2(T E)$-structures, which were subject of Theorem 3.29. The parameter space $\mathbb{P}^{1}$ of each $(T E)$-structure over $\mathbb{P}^{1}$ in Remarks $7.1(i)+(i i)$ embeds into one of these moduli spaces, after the choice of a marking. Also these embeddings will be described in Theorem 7.4.

Because of Corollary 7.3, any marked regular singular rank $2(T E)$-structure over a manifold $M$ is induced by a holomorphic map $M \rightarrow M^{\left(H^{\text {ref, } \infty}, M^{\text {ref }}\right) \text {,reg }}$, where $\left(H^{\text {ref, } \infty}, M^{\text {ref }}\right)$ is the reference pair used in the marking of the $(T E)$-structure. Remark 7.5 says something about the horizontal direction(s) in the moduli spaces. 


\section{Remarks 7.1.}

(i) Consider the manifold $M^{(3)}:=\mathbb{P}^{1}$ with coordinate $t_{2}$ on $\mathbb{C} \subset \mathbb{P}^{1}$ and coordinate $\widetilde{t}_{2}:=t_{2}^{-1}$ on $\mathbb{P}^{1} \backslash\{0\} \subset \mathbb{P}^{1}$.

With the projection $M^{(3)} \rightarrow\{0\}$, we pull back the flat bundle $H^{\prime} \rightarrow \mathbb{C}^{*}$ in Theorem 4.17 to a flat bundle $H^{(3)^{\prime}}$ on $\mathbb{C}^{*} \times M$. Recall the notations (4.4).

Now we read $\underline{v}:=\left(s_{1}+t_{2} s_{2}, z s_{2}\right)$ in $(4.25)$ and $(4.27)$ as a basis of sections on $\left.H^{(3)^{\prime}}\right|_{\mathbb{C}^{*} \times \mathbb{C}}$, and $\underline{\widetilde{v}}:=\left(s_{2}+\widetilde{t}_{2} s_{1}, z s_{1}\right)$ in $(4.26)$ and $(4.19)$ as a basis of sections on $\left.H^{(3)^{\prime}}\right|_{\mathbb{C}^{*} \times\left(\mathbb{P}^{1} \backslash\{0\}\right)}$. One sees immediately

$$
z \nabla_{\partial_{2}} \underline{v}=\underline{v} C_{2}, \quad z \nabla_{\widetilde{\partial}_{2}} \underline{\widetilde{v}}=\underline{\widetilde{v}} C_{2} .
$$

and again (4.25) resp. (4.27) and (4.26) resp. (4.19). Therefore $\underline{v}$ and $\underline{\widetilde{v}}$ are in any case bases of a $(T E)$-structure $\left(H^{(3)} \rightarrow \mathbb{C} \times M^{(3)}, \nabla^{(3)}\right)$ on $\mathbb{C} \times \mathbb{C} \subset \mathbb{C} \times M^{(3)}$ respectively $\mathbb{C} \times\left(\mathbb{P}^{1} \backslash\{0\}\right) \subset \mathbb{C} \times M^{(3)}$. The restricted $(T E)$-structures over $t_{2} \in \mathbb{C}^{*}$ are those in Theorem 4.17. They are regular singular, but not logarithmic. Their leadings exponents $\alpha_{1}$ and $\alpha_{2}$ are independent of $t_{2} \in \mathbb{C}^{*}$. The $(T E)$-structures over $t_{2}=0$ and over $\widetilde{t}_{2}=0$ (so $t_{2}=\infty$ ) are logarithmic except for the case $\left(N^{\text {mon }} \neq 0\right.$ and $\left.\alpha_{1}=\alpha_{2}\right)$, in which case the one over $t_{2}=0$ is regular singular, but not logarithmic. Their leading exponents are called $\alpha_{1}^{0}$ and $\alpha_{2}^{0}$ and $\alpha_{1}^{\infty}$ and $\alpha_{2}^{\infty}$. Then

\begin{tabular}{c|c}
\hline \multicolumn{1}{c|}{ over 0} & over $\infty$ \\
\hline$\alpha_{1}^{0}=\alpha_{1}$ & $\alpha_{1}^{\infty}=\alpha_{1}+1$ \\
$\alpha_{2}^{0}=\alpha_{2}+1$ & $\alpha_{2}^{\infty}=\alpha_{2}$ \\
\hline
\end{tabular}

except that in the case $\left(N^{\text {mon }} \neq 0\right.$ and $\left.\alpha_{1}=\alpha_{2}\right)$ we have $\alpha_{1}^{0}=\alpha_{1}, \alpha_{2}^{0}=\alpha_{2}$. For use in Theorem 7.3, we write the base space for the $(T E)$-structure over $\mathbb{P}^{1}$ with leading exponents $\alpha_{1}$ and $\alpha_{2}$ as $M^{(3), 0, \alpha_{1}, \alpha_{2}} \cong \mathbb{P}^{1}$ in the case $N^{\text {mon }}=0$ and as $M^{(3), \neq 0, \alpha_{1}, \alpha_{2}} \cong \mathbb{P}^{1}$ in the case $N^{\text {mon }} \neq 0$.

(ii) We extend the case $N^{\text {mon }}=0$ from Theorem $4.17(a)$ to the case $\alpha_{1}=\alpha_{2}$. (4.25) and (4.26) still hold, but now the restricted $(T E)$-structures over points in $M^{(3)}=\mathbb{P}^{1}$ are all logarithmic, though the $(T E)$-structure over $M^{(3)}$ is not logarithmic, but only regular singular. (7.1) still holds. In this case, the leading exponents are constant and are $\alpha_{1}$ and $\alpha_{1}+1$ (so, not $\alpha_{1}$ and $\alpha_{2}=\alpha_{1}$ ). Similarly to $(i)$, the base space is called $M^{(3), 0, \alpha_{1}, \log } \cong \mathbb{P}^{1}$.

\section{Remarks 7.2.}

(i) The construction in Lemma $3.10(d)$ extends a $(T E)$-structure $\left(H^{(3)} \rightarrow \mathbb{C} \times M^{(3)}, \nabla^{(3)}\right)$ in Remark $7.1(i)$ or $(i i)$ with $M^{(3)}=\mathbb{P}^{1}$ to a $(T E)$-structure $\left(H^{(4)} \rightarrow \mathbb{C} \times M^{(4)}, \nabla^{(4)}\right)$ with $M^{(4)}=\mathbb{C} \times M^{(3)}=\mathbb{C} \times \mathbb{P}^{1}$, via $\left(\mathcal{O}\left(H^{(4)}\right), \nabla^{(4)}\right)=\left(\varphi^{(4)}\right)^{*}\left(\mathcal{O}\left(H^{(3)}\right), \nabla^{(3)}\right) \otimes \mathcal{E}^{t_{1} / z}$, where $t_{1}$ is the coordinate on the first factor $\mathbb{C}$ in $\mathbb{C} \times \mathbb{P}^{1}$, and where $\varphi^{(4)}: M^{(4)} \rightarrow M^{(3)}$, $\left(t_{1}, t_{2}\right) \mapsto t_{2}$, is the projection. Define $\underline{v^{(4)}}:=\left(\varphi^{(4)}\right)^{*}(\underline{v}$ in Remark 7.1(i) or $(i i))$.

Then the matrices $A_{2}$ and $B$ with $z \nabla_{\partial_{i}} \underline{v}^{(4)}=\underline{v}^{(4)} A_{i}$ and $z^{2} \nabla_{\partial_{z}} \underline{v}^{(4)}=\underline{v}^{(4)} B$ are unchanged, and $A_{1}=C_{1}$, so

$$
A_{1}=C_{1}, \quad A_{2}=C_{2} \quad(\text { as in }(7.1)), \quad B \quad \text { is as in (4.25) or (4.27). }
$$

The Higgs field is everywhere on $M^{(4)}$ primitive. By Theorem $3.14, M^{(4)}=\mathbb{C} \times \mathbb{P}^{1}$ is an $F$ manifold with Euler field. The unit field is $\partial_{1}$, the multiplication is given by $\partial_{2} \circ \partial_{2}=0$ 
and $\widetilde{\partial}_{2} \circ \widetilde{\partial}_{2}=0$. So, each germ $\left(M^{(4)}, t^{0}\right)$ is the germ $\mathcal{N}_{2}$. The Euler field is

$$
\begin{aligned}
E= & t_{1} \partial_{1}+\left(\alpha_{1}-\alpha_{2}\right) t_{2} \partial_{2}=t_{1} \partial_{1}+\left(\alpha_{2}-\alpha_{1}\right) \widetilde{t}_{2} \widetilde{\partial}_{2} \\
& \left\{\begin{array}{l}
\text { in the case }(4.25) \text { and }(4.26) \text { and in }(i i) \text { above, } \\
\text { and in the case }(4.27) \text { and }(4.19) \text { with } \alpha_{1}-\alpha_{2} \in \mathbb{N},
\end{array}\right. \\
E= & t_{1} \partial_{1}-\partial_{2}=t_{1} \partial_{1}+\widetilde{t}_{2}^{2} \widetilde{\partial}_{2} \quad \text { in the case (4.27) and (4.19) with } \alpha_{1}=\alpha_{2} .
\end{aligned}
$$

(ii) For $t^{(4)} \in \mathbb{C} \times \mathbb{C}^{*} \subset M^{(4)}$, the $(T E)$-structure $\left(H^{(4)} \rightarrow \mathbb{C} \times\left(M^{(4)}, t^{(4)}\right), \nabla\right)$ is a universal unfolding of the one over $t^{0}$, because that one is of type (Reg) and because the Higgs field is primitive. See Corollary 5.1.

(iii) Let $\left(H \rightarrow \mathbb{C} \times\left(M, t^{0}\right), \nabla\right)$ be a regular singular unfolding of a regular singular, but not logarithmic rank $2(T E)$-structure over $t^{0}$. Because of part (ii), it is induced by the $(T E)$ structure $\left(H^{(4)}, \nabla^{(4)}\right)$ via a map $\left(M, t^{0}\right) \rightarrow\left(M^{(4)}, t^{(4)}\right)$ for some $t^{(4)} \in\{0\} \times \mathbb{C}^{*}$. Because it is regular singular, the image of the map is in $\{0\} \times \mathbb{C}^{*} \subset\{0\} \times M^{(3)}$. As there the leading exponents are constant, they are also constant on the unfolding $(H, \nabla)$.

Theorem 7.3. Any marked regular singular rank 2 (TE)-structure (see Definition 3.15(b), especially, $M$ is simply connected) is a good family of marked regular singular (TE)-structures (over points) in the sense of Definition 3.26(b).

Proof. Let $((H \rightarrow \mathbb{C} \times M, \nabla), \psi)$ be a regular singular rank $2(T E)$-structure with a marking $\psi$, i.e., an isomorphism $\psi$ from $\left(H^{\infty}, M^{\text {mon }}\right)$ to a reference pair $\left(H^{\text {ref, } \infty}, M^{\text {ref }}\right)$. We have to show the conditions (3.21) and (3.22) for a good family of marked regular singular (TE)-structures.

By definition of a marking, $M$ is simply connected, so especially it is connected. The subset

$$
M^{[\log ]}:=\left\{t \in M|\mathcal{U}|_{t}=0\right\}=\{t \in M \mid \text { the }(T E) \text {-structure over } t \text { is logarithmic }\}
$$

is a priori a subvariety (in fact, it is either $\varnothing$ or a hypersurface or equal to $M$ ).

First consider the case $M^{[\mathrm{log}]}=M$. Choose any point $t^{0} \in M$ and any disk $\Delta \subset M$ through $t^{0}$. The restriction of the (TE)-structure over the germ $\left(\Delta, t^{0}\right)$ is in the case $N^{\text {mon }}=0$ isomorphic to one in the 7 th or 8 th or 9 th case in Theorem 6.3. In the case $N^{\text {mon }} \neq 0$, it is isomorphic to one in case (III) in Theorem 6.7. In either case the leading exponents are constant on $\Delta$, because of table (6.13) in Remark 6.4(iv) and because of the definition of case (III) in Theorem 6.7. Therefore they are constant on $M$. We call them $\alpha_{1}^{\text {gen }}$ and $\alpha_{2}^{\text {gen }}$.

Now consider the case $M^{[\log ]} \varsubsetneqq M$. For each $t^{0} \in M \backslash M^{[\log ]}$, the $(T E)$-structure over the germ $\left(M, t^{0}\right)$ has constant leading exponents because of Remark 7.2(iii). Therefore the leading exponents are constant on $M \backslash M^{[\log ]}$. We call these generic leading exponents $\alpha_{1}^{\text {gen }}$ and $\alpha_{2}^{\text {gen }}$.

For $t^{0} \in M^{[\log ]}$ choose a generic small disk $\Delta \subset M$ through $t^{0}$. Then $\Delta \backslash\left\{t^{0}\right\} \subset M \backslash M^{[\log ]}$. The restriction of the (TE)-structure over the germ $\left(\Delta, t^{0}\right)$ is in the case $N^{\text {mon }}=0$ isomorphic to one in the 5 th case in Theorem 6.3. In the case $N^{\text {mon }} \neq 0$, it is isomorphic to one in case (I) or case (II) in Theorem 6.7. In either case, the leading exponents $\left(\alpha_{1}\left(t^{0}\right), \alpha_{2}\left(t^{0}\right)\right)$ of the $(T E)$-structure over $t^{0}$ are either $\left(\alpha_{1}^{\text {gen }}+1, \alpha_{2}^{\text {gen }}\right)$ or $\left(\alpha_{1}^{\text {gen }}, \alpha_{2}^{\text {gen }}+1\right)$, because of table $(6.13)$ in Remark 6.4(iv) and because of the definition of the cases (I) and (II) in Theorem 6.7.

Remark 6.4(iv) and Theorem 6.7 provide generators of $\mathcal{O}\left(\left.H\right|_{\mathbb{C} \times\left(\Delta, t^{0}\right)}\right)_{\left(0, t^{0}\right)}$ which are certain linear combinations of elementary sections. The shape of these generators and the almost constancy of the leading exponents imply the two conditions,

$$
\begin{aligned}
& \mathcal{O}\left(\left.H\right|_{\mathbb{C} \times\{t\}}\right)_{(0, t)} \supset V^{r} \quad \text { for any } t \in M, \text { where } r:=\max \left(\operatorname{Re}\left(\alpha_{1}^{\text {gen }}\right)+1, \operatorname{Re}\left(\alpha_{2}^{\text {gen }}\right)+1\right), \\
& \operatorname{dim}_{\mathbb{C}} \mathcal{O}\left(\left.H\right|_{\mathbb{C} \times\{t\}}\right)_{(0, t)} / V^{r} \quad \text { is independent of } t \in M,
\end{aligned}
$$

which are the conditions (3.21) and (3.22) for a good family of marked regular singular (TE)structures. 
The following theorem describes the moduli space $M^{\left(H^{\mathrm{ref}, \infty}, M^{\mathrm{ref}}\right) \text {,reg }}$ from Theorem 3.29 for the marked regular singular rank $2(T E)$-structures as infinite unions of curves isomorphic to $\mathbb{P}^{1}$ such that the families of $(T E)$-structures over these curves are the $(T E)$-structures in Remarks $7.1(i)+(i i)$. Part $(a)$ treats the cases with $N^{\text {mon }}=0$, part $(b)$ treats the cases with $N^{\text {mon }} \neq 0$. Recall the definitions of $M^{(3), 0, \alpha_{1}, \alpha_{2}}, M^{(3), \neq 0, \alpha_{1}, \alpha_{2}}$ and $M^{(3), 0, \alpha_{1}, \log }$ in Remarks $7.1(i)$ and $(i i)$.

Theorem 7.4. Let $\left(H^{\mathrm{ref}, \infty}, M^{\mathrm{ref}}\right)$ be a reference pair with $\operatorname{dim} H^{\mathrm{ref}, \infty}=2$. Let $\operatorname{Eig}\left(M^{\mathrm{ref}}\right)=$ $\left\{\lambda_{1}, \lambda_{2}\right\}$ be the set of eigenvalues of $M^{\mathrm{ref}}$. Let $\beta_{1}, \beta_{2} \in \mathbb{C}$ be the unique numbers with $\mathrm{e}^{-2 \pi \mathrm{i} \beta_{j}}=\lambda_{j}$ and $-1<\operatorname{Re} \beta_{j} \leq 0$.

(a) The case $N^{\text {mon }}=0$.

(i) The cases with $\lambda_{1} \neq \lambda_{2}$. Then

$$
M^{\left(H^{\mathrm{ref}, \infty}, M^{\mathrm{ref}}\right), \mathrm{reg}}=\bigcup_{l_{1} \in \mathbb{Z}}\left(\bigcup_{l_{2} \in \mathbb{Z}} M^{(3), 0, \beta_{1}+l_{1}+l_{2}, \beta_{2}-l_{2}}\right) .
$$

Its topological components are the unions in brackets, so $\bigcup_{l_{2} \in \mathbb{Z}} M^{(3), 0, \beta_{1}+l_{1}+l_{2}, \beta_{2}-l_{2}}$. Each component is a chain of $\mathbb{P}^{1}$ 's, the point $\infty$ of $M^{(3), 0, \alpha_{1}, \alpha_{2}}$ is identified with the point 0 of $M^{(3), 0, \alpha_{1}+1, \alpha_{2}-1}$.

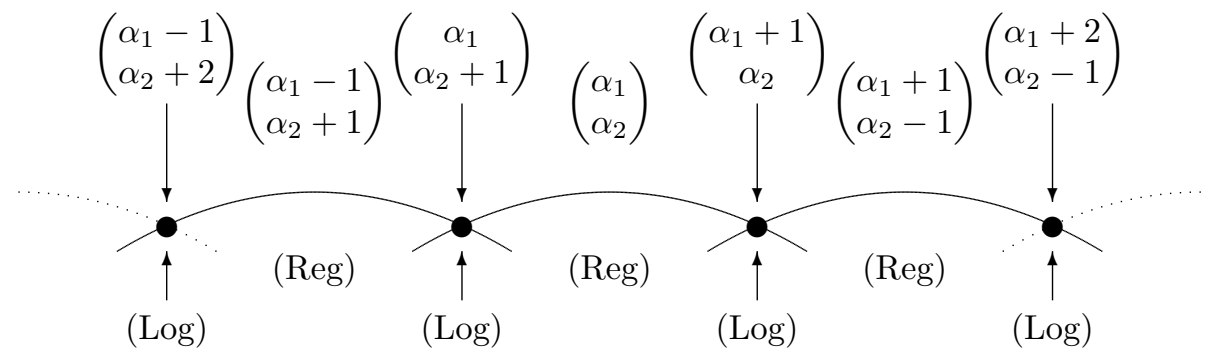

Figure 7.1. One topological component in part $(a)(i)$.

(ii) The cases with $\lambda_{1}=\lambda_{2}\left(\right.$ so $\left.\beta_{1}=\beta_{2}\right)$. Then

$$
\begin{aligned}
M^{\left(H^{\mathrm{ref}, \infty}, M^{\mathrm{ref}}\right), \mathrm{reg}}= & \bigcup_{l_{1} \in \mathbb{Z}}\left(\bigcup_{l_{2} \in \mathbb{N}} \mathbb{F}_{2}^{\beta_{1}+l_{1}+l_{2}, \beta_{1}+l_{1}-l_{2}}\right) \\
& \cup \bigcup_{l_{1} \in \mathbb{Z}}\left(\widetilde{\mathbb{F}}_{2}^{\beta_{1}+l_{1}+1, \beta_{1}+l_{1}} \cup \bigcup_{l_{2} \in \mathbb{N}} \mathbb{F}_{2}^{\beta_{1}+l_{1}+1+l_{2}, \beta_{1}+l_{1}-l_{2}}\right) .
\end{aligned}
$$

Here $\mathbb{F}_{2}^{\alpha_{1}, \alpha_{2}}$ is for all possible $\alpha_{1}$, $\alpha_{2}$ the Hirzebruch surface $\mathbb{F}_{2}$, and $\widetilde{\mathbb{F}}_{2}^{\alpha_{1}, \alpha_{1}-1}$ is the surface $\widetilde{\mathbb{F}}_{2}$, which is obtained from $\mathbb{F}_{2}$ by blowing down the unique (-2)-curve in $\mathbb{F}_{2}$. The unions in brackets are the topological components. They are chains of Hirzebruch surfaces. $A(+2)$-curve of $\mathbb{F}_{2}^{\alpha_{1}, \alpha_{2}}$ is identified with the $(-2)$-curve of $\mathbb{F}_{2}^{\alpha_{1}+1, \alpha_{2}-1}$ (and $a(+2)$-curve of $\widetilde{\mathbb{F}}_{2}^{\alpha_{1}, \alpha_{1}-1}$ is identified with the $(-2)$ curve in $\left.\mathbb{F}_{2}^{\alpha_{1}+1, \alpha_{1}-2}\right)$. The $(T E)$ structures over the points in the (-2)-curves are logarithmic, and also the (TE)structure over the singular point of $\widetilde{\mathbb{F}}_{2}^{\alpha_{1}, \alpha_{1}-1}$ is logarithmic. The (TE)-structures over all other points of $\mathbb{F}_{2}^{\alpha_{1}, \alpha_{2}}$ and $\widetilde{\mathbb{F}}_{2}^{\alpha_{1}, \alpha_{2}}$ are regular singular, but not logarithmic, and have leading exponents $\alpha_{1}$ and $\alpha_{2}$. For each $\mathbb{F}_{2}^{\alpha_{1}, \alpha_{2}}$, and also for $\widetilde{\mathbb{F}}_{2}^{\alpha_{1}, \alpha_{2}}$ after blowing up the singular point to a (-2)-curve, the fibers of it as a $\mathbb{P}^{1}$-fiber bundle over $\mathbb{P}^{1}$ are 
isomorphic to $M^{(3), 0, \alpha_{1}, \alpha_{2}}$. The $(-2)$-curve in $\mathbb{F}_{2}^{\alpha_{1}, \alpha_{1}-2}$ (the $\mathbb{F}_{2}$ with $l_{2}=1$ in each topological component in the first line of (7.2)) is isomorphic to $M^{(3), 0, \alpha_{1}-1, \log \text {, and }}$ the (TE)-structures over its points are logarithmic with leading exponents $\alpha_{1}, \alpha_{1}-1$.

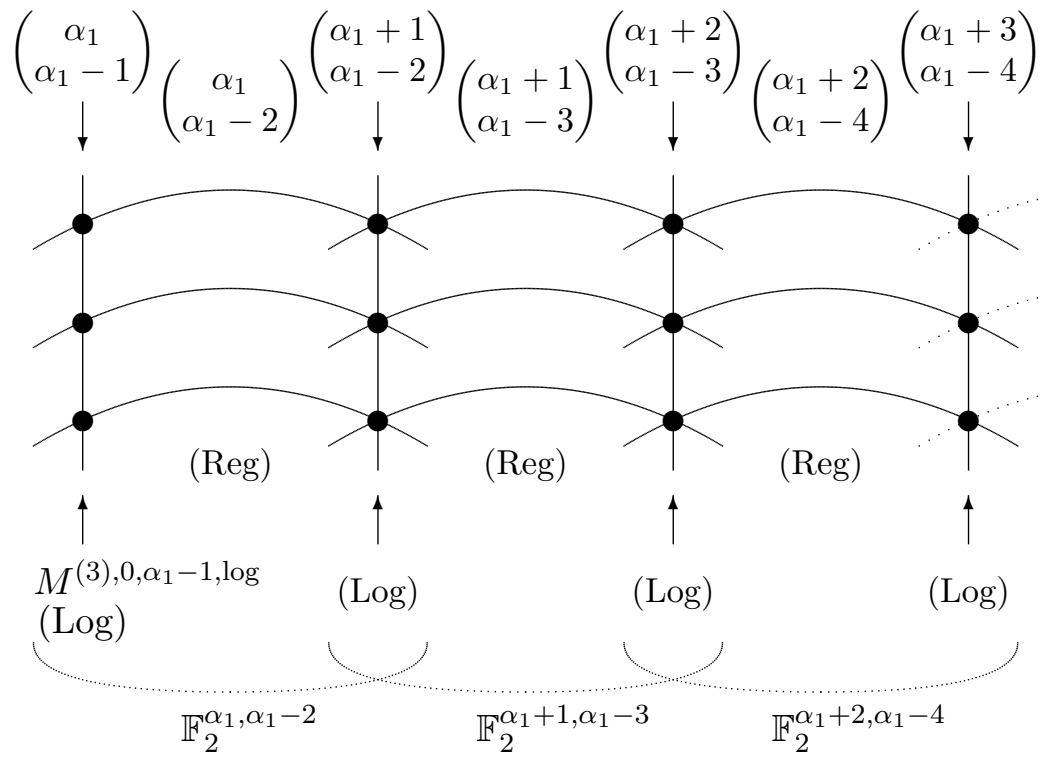

Figure 7.2. One topological component in part (a) (ii).

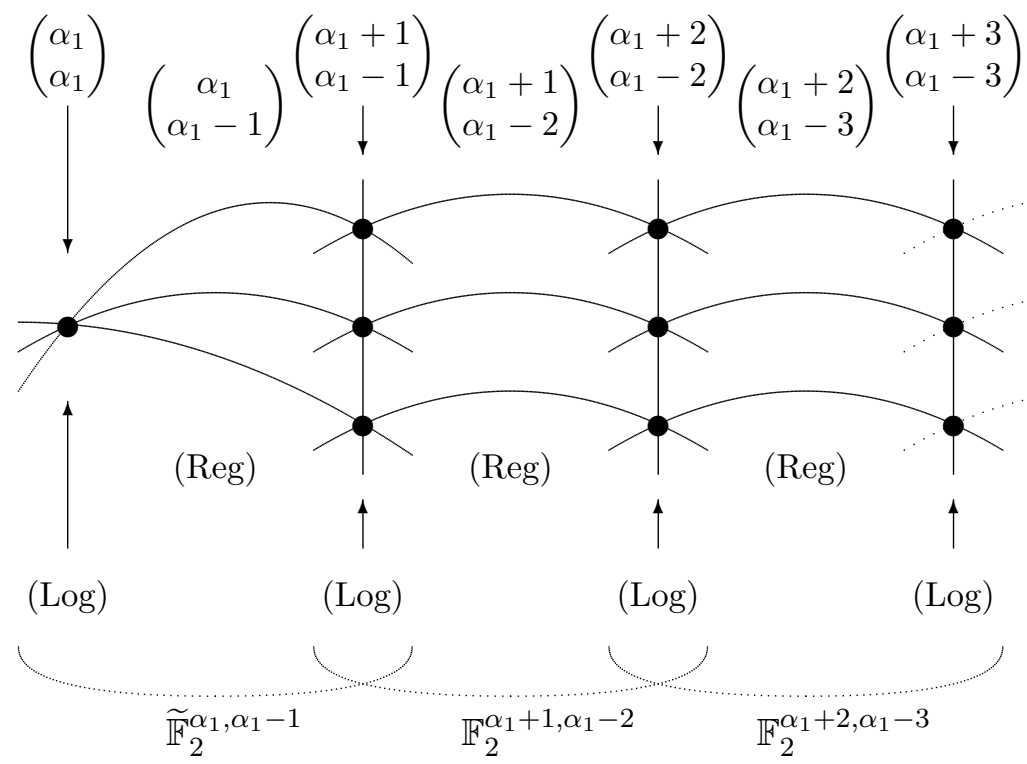

Figure 7.3. Another topological component in part (a) (ii).

(b) The cases with $N^{\text {mon }} \neq 0$ (and thus $\lambda_{1}=\lambda_{2}, \beta_{1}=\beta_{2}$ ). Then

$$
\begin{aligned}
M^{\left(H^{\mathrm{ref}, \infty}, M^{\mathrm{ref}}\right), \mathrm{reg}}= & \bigcup_{l_{1} \in \mathbb{Z}}\left(\bigcup_{l_{2} \in \mathbb{N}_{0}} M^{(3), \neq 0, \beta_{1}+l_{1}+l_{2}, \beta_{1}+l_{1}-l_{2}}\right) \\
& \cup \bigcup_{l_{1} \in \mathbb{Z}}\left(\bigcup_{l_{2} \in \mathbb{N}_{0}} M^{(3), \neq 0, \beta_{1}+l_{1}+1+l_{2}, \beta_{1}+l_{1}-l_{2}}\right) .
\end{aligned}
$$


Its topological components are the unions in brackets. Each component is a chain of $\mathbb{P}^{1}$ 's, the point $\infty$ of $M^{(3), \neq 0, \alpha_{1}, \alpha_{2}}$ is identified with the point 0 of $M^{(3), \neq 0, \alpha_{1}+1, \alpha_{2}-1}$.

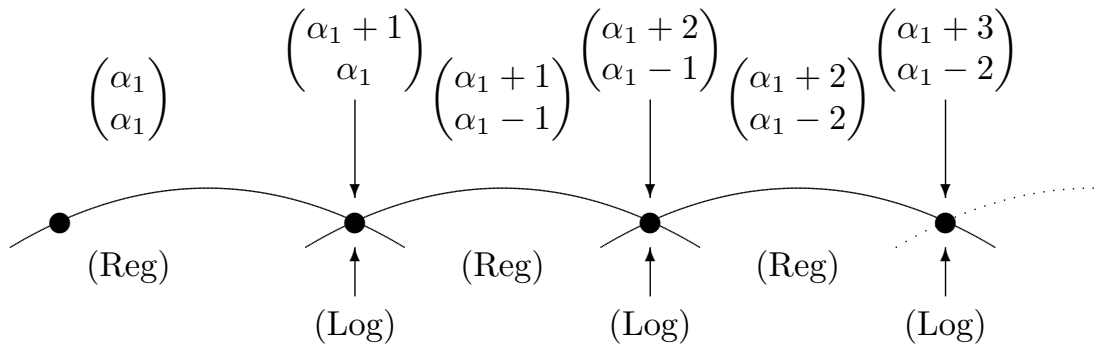

Figure 7.4. One topological component in part (b).

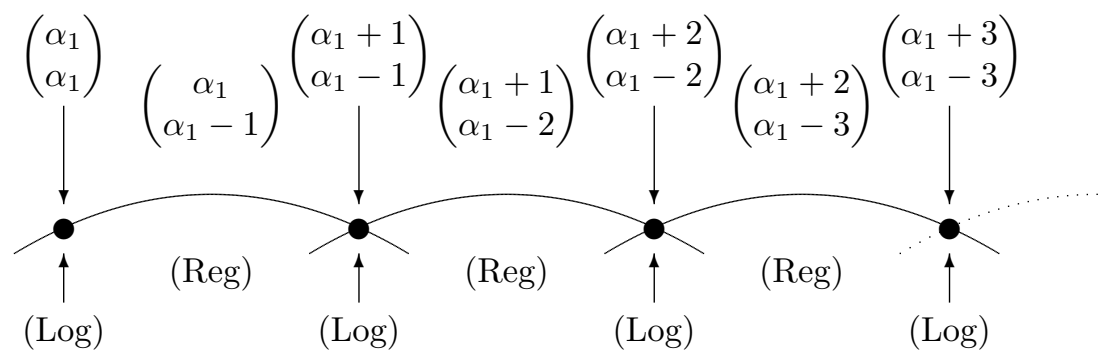

Figure 7.5. Another topological component in part (b).

Proof. We consider only marked $(T E)$-structures with a fixed reference pair $\left(H^{\text {ref, } \infty}, M^{\text {ref }}\right)$. Because of the markings, we can identify for each such $(T E)$-structure its pair $\left(H^{\infty}, M^{\text {mon }}\right)$ with the reference pair $\left(H^{\mathrm{ref}, \infty}, M^{\mathrm{ref}}\right)$. Thus also the spaces $C^{\alpha}$ can be identified for all marked $(T E)$-structures.

(a) $(i)$ and (b) In both parts, there is no harm in fixing elementary sections $s_{1} \in C^{\alpha_{1}}$ and $s_{2} \in C^{\alpha_{2}}$ as in Theorem 4.17. Then Theorem 4.17 lists all marked (TE)-structures with the given reference pair. Remarks 7.1 $(i)$ just put these marked $(T E)$-structures into families parametrized by the spaces $M^{(3), 0, \alpha_{1}, \alpha_{2}}$ resp. $M^{(3), \neq 0, \alpha_{1}, \alpha_{2}}$. Most logarithmic $(T E)$-structures (which are classified in Theorem 4.20) turn up in two such families. This leads to the identification of the point $\infty$ in $M^{(3), 0 / \neq 0, \alpha_{1}, \alpha_{2}}$ with the point 0 in $M^{(3), 0 / \neq 0, \alpha_{1}+1, \alpha_{2}-1}$. Only each of the logarithmic $(T E)$-structures with $N^{\text {mon }} \neq 0$ and leading exponents $\alpha_{1}=\alpha_{2}$ turns up in only one $\mathbb{P}^{1}$, in the space $M^{(3), \neq 0, \alpha_{1}, \alpha_{1}-1}$. There it is over the point 0 .

(a) (ii) Here the leading exponents satisfy $\alpha_{1}-\alpha_{2} \in \mathbb{Z} \backslash\{0\}$, and we index them such that $\alpha_{1}-\alpha_{2} \in \mathbb{N}$. We fix a basis $\sigma_{1}, \sigma_{2}$ of $C^{\alpha_{1}}$ and define $\sigma_{3}:=z^{\alpha_{2}-\alpha_{1}} \sigma_{1} \in C^{\alpha_{2}}, \sigma_{4}=z^{\alpha_{2}-\alpha_{1}} \sigma_{2} \in C^{\alpha_{2}}$. Then because of Theorem 4.17(a), we can write all marked regular singular, but not logarithmic $(T E)$-structures with leading exponents $\alpha_{1}$ and $\alpha_{2}$ in two charts $\mathbb{C} \times \mathbb{C}^{*}$ with coordinates $\left(r_{1}, t_{2}\right)$ and $\left(r_{2}, t_{3}\right)$,

$$
\begin{aligned}
& \mathcal{O}(H)_{0}=\mathbb{C}\{z\}\left(\sigma_{1}+t_{2}\left(\sigma_{4}+r_{1} \sigma_{3}\right)\right) \oplus \mathbb{C}\{z\}\left(z\left(\sigma_{4}+r_{1} \sigma_{3}\right)\right), \\
& \mathcal{O}(H)_{0}=\mathbb{C}\{z\}\left(\sigma_{2}+t_{3}\left(\sigma_{3}+r_{2} \sigma_{4}\right)\right) \oplus \mathbb{C}\{z\}\left(z\left(\sigma_{3}+r_{2} \sigma_{4}\right)\right) .
\end{aligned}
$$

The charts overlap where $r_{1}, r_{2} \in \mathbb{C}^{*}$, with

$$
r_{2}=r_{1}^{-1}, \quad t_{3}=-t_{2} r_{1}^{2}
$$

Compactification to $t_{2}=0$ and $t_{2}=\infty$ (and $t_{3}=0$ and $t_{3}=\infty$ ) gives the Hirzebruch surface $\mathbb{F}_{2}=\mathbb{F}_{2}^{\alpha_{1}, \alpha_{2}}$. The curve with $t_{2}=0$ (and $t_{3}=0$ ) is the $(-2)$-curve. Over this curve, we have 
the family of marked logarithmic (TE)-structures (see Theorem 4.20) with leading exponents $\alpha_{1}$ and $\alpha_{2}+1$,

$$
\begin{aligned}
& \mathcal{O}(H)_{0}=\mathbb{C}\{z\}\left(\sigma_{1}\right) \oplus \mathbb{C}\{z\}\left(z\left(\sigma_{4}+r_{1} \sigma_{3}\right)\right), \\
& \mathcal{O}(H)_{0}=\mathbb{C}\{z\}\left(\sigma_{2}\right) \oplus \mathbb{C}\{z\}\left(z\left(\sigma_{3}+r_{2} \sigma_{4}\right)\right) .
\end{aligned}
$$

The curve with $t_{2}=\infty$ (and $\left.t_{3}=\infty\right)$ is a $(+2)$-curve. Over this curve, we have the family of marked logarithmic (TE)-structures with leading exponents $\alpha_{1}+1$ and $\alpha_{2}$. Therefore the $(+2)$-curve in $\mathbb{F}_{2}^{\alpha_{1}, \alpha_{2}}$ must be identified with the $(-2)$-curve in $\mathbb{F}_{2}^{\alpha_{1}+1, \alpha_{2}-1}$.

In the case $\alpha_{1}-\alpha_{2}=2$, the $(-2)$-curve in $\mathbb{F}_{2}^{\alpha_{1}, \alpha_{2}}$ is the moduli space $M^{(3), 0, \alpha_{1}-1, \log }$ from Remark 7.1(i).

In the case $\alpha_{1}-\alpha_{2}=1$, the $(-2)$-curve in $\mathbb{F}_{2}^{\alpha_{1}, \alpha_{2}}$ has to be blown down, as then for $t_{2}=0$

$$
\mathcal{O}(H)_{0}=\mathbb{C}\{z\}\left(\sigma_{1}\right) \oplus \mathbb{C}\{z\}\left(z\left(\sigma_{4}+r_{1} \sigma_{3}\right)\right)=\mathbb{C}\{z\} C^{\alpha_{1}}=V^{\alpha_{1}}
$$

is independent of the parameter $r_{1}$.

The projection $\left(r_{1}, t_{2}\right) \mapsto r_{1}$ extends to the $\mathbb{P}^{1}$-fibration of $\mathbb{F}_{2}^{\alpha_{1}, \alpha_{2}}$ over $\mathbb{P}^{1}$. The fibers are isomorphic to $M^{(3), 0, \alpha_{1}, \alpha_{2}}$. Affine coordinates on these fibers are $t_{2}$ and $\widetilde{t}_{2}=t_{2}^{-1}$ or $t_{3}$ and $\tilde{t}_{3}=t_{3}^{-1}$.

\section{Remarks 7.5.}

(i) Consider a marked regular singular rank 2 (TE)-structure $((H \rightarrow \mathbb{C} \times M, \nabla), \psi)$. There is a unique map $\varphi: M \rightarrow M^{\left(H^{\text {ref, } \infty}, M^{\text {ref }}\right) \text {,reg }}$, which maps $t \in M$ to the unique point in $M^{\left(H^{\text {ref, } \infty}, M^{\text {ref }}\right) \text {,reg }}$ over which one has up to marked isomorphism the same marked $(T E)$ structure as over $t$. Corollary 7.3 and the fact that the moduli space represents the moduli functor $\mathcal{M}^{\left(H^{\text {ref }, \infty}, M^{\text {ref }}\right) \text {,reg }}$, imply that $\varphi$ is holomorphic.

Because $M$ is (simply) connected, the map $\varphi$ goes to one irreducible component of the moduli space, so to one $M^{(3), 0 / \neq 0, \alpha_{1}, \alpha_{2}} \cong \mathbb{P}^{1}$ in the parts $(a)(i)$ and $(b)$ in Theorem 7.4 and to one $\mathbb{F}_{2}^{\alpha_{1}, \alpha_{2}}$ or to $\widetilde{\mathbb{F}}_{2}^{\alpha_{1}, \alpha_{1}-1}$ in part $(a)(i i)$.

(ii) In fact, in part $(a)$ ( $i i)$ the map $\varphi$ goes even to a projective curve which is isomorphic to one curve $M^{(3), 0, \alpha_{1}, \alpha_{2}}$ or to the curve $M^{(3), 0, \alpha_{1}, \log }$. This holds for the (TE)-structure over any manifold, as it holds by Remark $6.4(\mathrm{iv})$ and Theorem 6.7 for the (TE)-structures over the 1-dimensional germ $(\mathbb{C}, 0)$.

The curves isomorphic to $M^{(3), 0, \alpha_{1}, \alpha_{2}}$ are the (0)-curves in the $\mathbb{P}^{1}$ fibration of $\mathbb{F}_{2}^{\alpha_{1}, \alpha_{2}}$ over $\mathbb{P}^{1}$ (in the case of $\widetilde{\mathbb{F}}_{2}^{\alpha_{2}+1, \alpha_{2}}$ each fiber of $\mathbb{F}_{2}^{\alpha_{2}+1, \alpha_{2}}$ over $\mathbb{P}^{1}$ embeds also into the blown down surface $\left.\widetilde{\mathbb{F}}_{2}^{\alpha_{2}+1, \alpha_{2}}\right)$. The curve isomorphic to $M^{(3), 0, \alpha_{1}, \log }$ is the $(-2)$-curve in $\mathbb{F}_{2}^{\alpha_{1}, \alpha_{1}-2}$.

(iii) We have here a notion of horizontal directions which is similar to that for classifying spaces of Hodge structures. There it comes from Griffiths transversality. Here it comes from the part of the pole of Poincaré rank 1, which says that the covariant derivatives $\nabla_{\partial_{j}}$ along vector fields on the base space see only a pole of order 1 .

In the cases of the $\mathbb{F}_{2}^{\alpha_{1}, \alpha_{2}}$ with $\alpha_{1}-\alpha_{2} \in \mathbb{N} \backslash\{1,2\}$, the horizontal directions are the tangent spaces to the fibers of the $\mathbb{P}^{1}$ fibration. In the cases of $\mathbb{F}_{2}^{\alpha_{1}, \alpha_{1}-2}$ and $\widetilde{\mathbb{F}}_{2}^{\alpha_{1}, \alpha_{1}-1}$, the horizontal directions contain these tangent spaces. However, on points in the (-2)-curve in $\mathbb{F}_{2}^{\alpha_{1}, \alpha_{1}-2}$ and on the singular point in $\widetilde{\mathbb{F}}_{2}^{\alpha_{1}, \alpha_{1}-1}$, any direction is horizontal.

Remark 7.6. If we forget the markings of the $(T E)$-structures in one moduli space $M^{\left(H^{\mathrm{ref}, \infty}, M^{\mathrm{ref}}\right), \text { reg }}$ and consider the unmarked $(T E)$-structures up to isomorphism, we obtain in the cases $N^{\text {mon }}=0$ countably many points, one for each intersection point or intersection curve of two irreducible components, and one for each irreducible component. On the contrary, 
in the cases $N^{\text {mon }} \neq 0$, the unmarked and the marked $(T E)$-structures almost coincide, as the choice of an elementary section $s_{1}$ in Theorem 4.17(b) fixes uniquely the elementary section $s_{2}$ with (4.22). The set of unmarked ( $T E$ )-structures up to isomorphism is still almost in bijection with the moduli space $M^{\left(H^{\mathrm{ref}, \infty}, M^{\mathrm{ref}}\right) \text {,reg }}$ in the case $N^{\text {mon }} \neq 0$. Only the components $M^{(3), \neq 0, \alpha_{1}, \alpha_{1}}-\{\infty\}$ boil down to single points.

\section{Unfoldings of rank $2(T E)$-structures of type ( $\mathrm{Log})$ over a point}

Sections 5 and 8 together treat all rank $2(T E)$-structures over germs $\left(M, t^{0}\right)$ of manifolds. Section 5 treated the unfoldings of $(T E)$-structures of types (Sem) or (Bra) or (Reg) over $t^{0}$. Section 8 will treat the unfoldings of $(T E)$-structures of type $(\log )$ over $t^{0}$.

It builds on Section 6, which classified the unfoldings with trace free pole parts over $\left(M, t^{0}\right)=$ $(\mathbb{C}, 0)$ of a logarithmic rank $2(T E)$-structure over $t^{0}$ and on Section 7 , which treated arbitrary regular singular rank $2(T E)$-structures. Here Lemmata 3.10 and 3.11 are helpful. They allow to go from arbitrary $(T E)$-structures to $(T E)$-structures with trace free pole parts and vice versa.

Section 8.1 gives the classification results. Section 8.2 extracts from them a characterization of the space of all ( $T E$ )-structures with generically primitive Higgs fields over a given germ of a 2-dimensional $F$-manifold with Euler field. Section 8.3 gives the proof of Theorem 8.5.

First we characterize in Theorem 8.1 the 2-parameter unfoldings of rank $2(T E)$-structures of type (Log) over a point such that the Higgs field is generically primitive and induces an $F$-manifold structure on the underlying germ $\left(M, t^{0}\right)$ of a manifold. Theorem 8.1 is a rather immediate implication of Theorem 6.3 and Theorem 6.7 together with Lemmata 3.10 and 3.11. Part $(d)$ gives an explicit classification. The other results in this section will all refer to this classification.

Corollary 8.3 lists for any logarithmic rank $2(T E)$-structure over a point $t^{0}$ all unfoldings within the set of $(T E)$-structures in Theorem 8.1(a). The proof consists of inspection of the explicit classification in Theorem 8.1 $(d)$.

Theorem 8.5 is the main result of this section. It lists a finite subset of the unfoldings in Theorem 8.1 $(d)$ with the following property: Any unfolding of a rank $2(T E)$-structure of type (Log) over a point is induced by a $(T E)$-structure in this list. The $(T E)$-structures in the list turn out to be universal unfoldings of themselves.

The proof of Theorem 8.5 is long. It is deferred to Section 8.3. The results of Section 6 are crucial, especially Theorem 6.3 and Theorem 6.7 .

Finally, Theorem 8.6 lists the rank $2(T E)$-structures over a germ $\left(M, t^{0}\right)$ of a manifold such that the Higgs field is primitive (so that $\left(M, t^{0}\right)$ becomes a germ of an $F$-manifold with Euler field) and the restriction over $t^{0}$ is of type $(\mathrm{Log})$. This list turns out to be a sublist of the one in Theorem 8.5. Theorem 8.6 follows easily from Theorem 8.1.

Theorem 8.6 is also contained in the papers [6] and [7], the generic types (Bra), (Reg) and $(\mathrm{Log})$ are in [6], the generic type (Sem) is in [7]. The proofs there are completely different. They build on the formal classification of $(T)$-structures in [5].

\subsection{Classification results}

\section{Theorem 8.1.}

(a) Consider a rank $2(T E)$-structure $\left(H \rightarrow \mathbb{C} \times\left(M, t^{0}\right), \nabla\right)$ over a 2 -dimensional germ $\left(M, t^{0}\right)$ with restriction over $t^{0}$ of type $(\log )$, with generically primitive Higgs field, and such that the induced $F$-manifold structure on generic points of $M$ extends to all of $M$. 
There is a unique rank $2(T E)$-structure $\left(H^{[3]} \rightarrow \mathbb{C} \times(\mathbb{C}, 0), \nabla^{[3]}\right)$ over $(\mathbb{C}, 0)$ (with coordinate $\left.t_{2}\right)$ with trace free pole part, with nonvanishing Higgs field and with logarithmic restriction over $t_{2}=0$ such that $(\mathcal{O}(H), \nabla)$ arises from $\left(\mathcal{O}\left(H^{[3]}\right), \nabla^{[3]}\right)$ as follows. There are coordinates $t=\left(t_{1}, t_{2}\right)$ on $\left(M, t^{0}\right)$ such that $\left(M, t^{0}\right)=\left(\mathbb{C}^{2}, 0\right)$ and a constant $c_{1} \in \mathbb{C}$ such that

$$
(\mathcal{O}(H), \nabla) \cong \operatorname{pr}_{2}^{*}\left(\mathcal{O}\left(H^{[3]}\right), \nabla^{[3]}\right) \otimes \mathcal{E}^{\left(t_{1}+c_{1}\right) / z},
$$

where $\operatorname{pr}_{2}:\left(M, t^{0}\right) \rightarrow(\mathbb{C}, 0),\left(t_{1}, t_{2}\right) \mapsto t_{2}\left(\right.$ see Lemma $3.10(a)$ for $\left.\mathcal{E}^{\left(t_{1}+c_{1}\right) / z}\right)$.

The $(T E)$-structure $(H, \nabla)$ is of type $(\log )$ over $(\mathbb{C} \times\{0\}, 0)$ and of one generic type $($ Sem $)$ or $($ Bra $)$ or $(R e g)$ or $(\log )$ over $\left(\mathbb{C} \times \mathbb{C}^{*}, 0\right)$.

(b) Vice versa, if $\left(H^{[3]}, \nabla^{[3]}\right)$ is as in (a) and $c_{1} \in \mathbb{C}$, then the $(T E)$-structure $(\mathcal{O}(H), \nabla):=$ $\operatorname{pr}_{2}^{*}\left(\mathcal{O}\left(H^{[3]}\right), \nabla^{[3]}\right) \otimes \mathcal{E}^{\left(t_{1}+c\right) / z}$ over $\left(M, t^{0}\right)=\left(\mathbb{C}^{2}, 0\right)$ satisfies the properties in $(a)$.

(c) The rank $2(T E)$-structures $\left(H^{[3]}, \nabla^{[3]}\right)$ over $(\mathbb{C}, 0)$ with trace free pole part, nonvanishing Higgs field and logarithmic restriction over 0 are classified in Theorems 6.3 and 6.7. They are in suitable coordinates the first 7 of the 9 cases in the list in Theorem 6.3 and the cases (6.26) and (6.27) with $f=\frac{1}{k_{1}} t^{k_{1}}$ for some $k_{1} \in \mathbb{N}$ in Theorem 6.7. (Though here the 6th case in Theorem 6.3 is part of the cases (6.26) and (6.27) in Theorem 6.7.)

(d) The explicit classification of the $(T E)$-structures $(H, \nabla)$ in $(a)$ is as follows. There are coordinates $\left(t_{1}, t_{2}\right)$ such that $\left(M, t^{0}\right)=\left(\mathbb{C}^{2}, 0\right)$, and there is a $\mathbb{C}\{t, z\}$-basis $\underline{v}$ of $\mathcal{O}(H)_{0}$ whose matrices $A_{1}, A_{2}, B \in M_{2 \times 2}(\mathbb{C}\{t, z\})$ with $z \nabla_{\partial_{i}} \underline{v}=\underline{v} A_{i}, z^{2} \nabla_{\partial_{z}} \underline{v}=\underline{v} B$ are in the following list of normal forms. The normal form is unique. We always have

$$
A_{1}=C_{1} .
$$

Always $M$ is an F-manifold with Euler field in one of the normal forms in Theorems 2.2 and 2.3 (in the case $(i)$ the product $\partial_{2} \circ \partial_{2}$ is only almost in the normal form in Theorem 2.2; in the case (iii) with $\alpha_{4}=-1$ the Euler field is only almost in the normal form in Theorem 2.3).

(i) Generic type $\left(\right.$ Sem): invariants $k_{1}, k_{2} \in \mathbb{N}$ with $k_{2} \geq k_{1}, c_{1}, \rho^{(1)} \in \mathbb{C}, \zeta \in \mathbb{C}$ if $k_{2}-k_{1} \in 2 \mathbb{N}, \alpha_{3} \in \mathbb{R}_{\geq 0} \cup \mathbb{H}$ if $k_{1}=k_{2}$,

$$
\begin{aligned}
\gamma:= & \frac{2}{k_{1}+k_{2}}, \\
A_{2}= & \left\{\begin{array}{l}
-\gamma^{-1}\left(t_{2}^{k_{1}-1} C_{2}+t_{2}^{k_{2}-1} E\right) \quad \text { if } k_{2}-k_{1}>0 \text { is odd, } \\
-\gamma^{-1}\left(t_{2}^{k_{1}-1} C_{2}+\zeta t^{\left(k_{1}+k_{2}\right) / 2-1} D+\left(1-\zeta^{2}\right) t_{2}^{k_{2}-1} E\right) \quad \text { if } k_{2}-k_{1} \in 2 \mathbb{N}, \\
-\gamma^{-1} t_{2}^{k_{1}-1} D \quad \text { if } k_{2}=k_{1},
\end{array}\right. \\
B= & \left(-t_{1}-c_{1}+z \rho^{(1)}\right) C_{1}+\left(-\gamma t_{2}\right) A_{2}+ \begin{cases}z \frac{k_{1}-k_{2}}{2\left(k_{1}+k_{2}\right)} D & \text { if } k_{2}>k_{1}, \\
z \alpha_{3} D & \text { if } k_{2}=k_{1},\end{cases} \\
& F-\text { manifold } I_{2}\left(k_{1}+k_{2}\right)\left(\text { with } I_{2}(2)=A_{1}^{2}\right), \text { with } \partial_{2} \circ \partial_{2}=\gamma^{-2} t_{2}^{k_{1}+k_{2}} \cdot \partial_{1}, \\
E= & \left(t_{1}+c_{1}\right) \partial_{1}+\gamma t_{2} \partial_{2} \quad \text { Euler field. }
\end{aligned}
$$

(ii) Generic type (Bra): invariants $k_{1}, k_{2} \in \mathbb{N}, c_{1}, \rho^{(1)} \in \mathbb{C}$,

$$
\begin{aligned}
& \gamma:=\frac{1}{k_{1}+k_{2}}, \\
& A_{2}=-\gamma^{-1}\left(t_{2}^{k_{1}-1} C_{2}+t_{2}^{k_{1}+k_{2}-1} D-t_{2}^{k_{1}+2 k_{2}-1} E\right),
\end{aligned}
$$




$$
\begin{aligned}
B= & \left(-t_{1}-c_{1}+z \rho^{(1)}\right) C_{1}+\left(-\gamma t_{2}\right) A_{2}+z \frac{-k_{2}}{2\left(k_{1}+k_{2}\right)} D, \\
& \quad F \text {-manifold } \mathcal{N}_{2}, \text { with } \partial_{2} \circ \partial_{2}=0, \\
E= & \left(t_{1}+c_{1}\right) \partial_{1}+\gamma t_{2} \partial_{2} \quad \text { Euler field. }
\end{aligned}
$$

(iii) Generic type (Reg): invariants $c_{1}, \rho^{(1)} \in \mathbb{C}, \alpha_{4} \in \mathbb{C} \backslash\{-1\}$ if $N^{\text {mon }}=0, \alpha_{4} \in \mathbb{Z}$ if $N^{\text {mon }} \neq 0, k_{1} \in \mathbb{N}$ if $N^{\text {mon }}=0, \widetilde{k}_{1} \in \mathbb{N}$ if $N^{\text {mon }} \neq 0$ (with $k_{1}=\widetilde{k}_{1}$ if $\alpha_{4} \neq-1$, and $k_{1}=2 \widetilde{k}_{1}$ if $\left.\alpha_{4}=-1\right)$,

$$
\begin{aligned}
& \gamma:=\frac{1+\alpha_{4}}{k_{1}}
\end{aligned}
$$

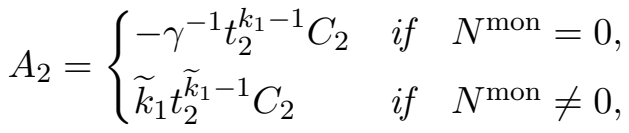

$$
\begin{aligned}
& B=\left(-t_{1}-c_{1}+z \rho^{(1)}\right) C_{1}+\left(-\gamma t_{2}\right) A_{2}+z \frac{1}{2} \alpha_{4} D \\
& +\left\{\begin{array}{l}
0 \quad \text { if } \quad N^{\text {mon }}=0, \\
z^{\alpha_{4}+1} C_{2} \quad \text { if } \quad N^{\text {mon }} \neq 0, \quad \alpha_{4} \in \mathbb{N}_{0}, \\
-z^{-\alpha_{4}-1} t_{2}^{2 \widetilde{k}_{1}} C_{2}+z^{-\alpha_{4}} t_{2}^{\widetilde{k}_{1}} D+z^{-\alpha_{4}+1} E \quad \text { if } \quad N^{\text {mon }} \neq 0, \quad \alpha_{4} \in \mathbb{Z}_{<0},
\end{array}\right. \\
& F \text {-manifold } \mathcal{N}_{2} \text {, with } \partial_{2} \circ \partial_{2}=0 \text {, } \\
& E=\left\{\begin{array}{lll}
\left(t_{1}+c_{1}\right) \partial_{1}+\gamma t_{2} \partial_{2} & \text { if } & \alpha_{4} \neq-1, \\
\left(t_{1}+c_{1}\right) \partial_{1}+\frac{1}{\widetilde{k}} t_{2}^{\kappa_{1}+1} \partial_{2} & \text { if } & \alpha_{4}=-1
\end{array}\right\} \quad \text { Euler field } .
\end{aligned}
$$

(iv) Generic type $\left(\right.$ Log): invariants $k_{1} \in \mathbb{N}, c_{1}, \rho^{(1)} \in \mathbb{C}$,

$$
\begin{aligned}
& A_{2}=k_{1} t_{2}^{k_{1}-1} C_{2}, \\
& B=\left(-t_{1}-c_{1}+z \rho^{(1)}\right) C_{1}-z \frac{1}{2} D, \quad \text { F-manifold } \mathcal{N}_{2}, \text { with } \partial_{2} \circ \partial_{2}=0, \\
& E=\left(t_{1}+c_{1}\right) \partial_{1} \quad \text { Euler field. }
\end{aligned}
$$

Theorem 8.1 is proved after Remark 8.2.

Remark 8.2. The other normal forms in Remark 6.6 for the generic type (Sem) with $k_{2}-k_{1} \in 2 \mathbb{N}$ and for the generic type (Bra) give the following other normal forms. In both cases, the formulas for $A_{1}=C_{1}, \gamma, B$, the $F$-manifold and $E$ are unchanged, only the matrix $A_{2}$ changes. For the generic type $(\mathrm{Sem})$ with $k_{2}-k_{1} \in 2 \mathbb{N}, A_{2}$ becomes

$$
A_{2}=-\gamma^{-1}\left(t_{2}^{k_{1}-1} C_{2}+t_{2}^{k_{2}-1} E\right)+z \frac{k_{2}-k_{1}}{2} \zeta t_{2}^{\left(k_{2}-k_{1}-2\right) / 2} E .
$$

For the generic type (Bra), $A_{2}$ becomes

$$
A_{2}=-\gamma^{-1} t_{2}^{k_{1}-1} C_{2}+z k_{2} t_{2}^{k_{2}-1} E \text {. }
$$

Proof of Theorem 8.1. We prove the parts of Theorem 8.1 in the order $(c),(d),(b),(a)$.

$(c)$ Consider a rank $2(T E)$-structure $\left(H^{[3]} \rightarrow \mathbb{C} \times(\mathbb{C}, 0), \nabla^{[3]}\right)$ (with coordinate $t_{2}$ on $(\mathbb{C}, 0)$ ) with trace free pole part and with logarithmic restriction over $t_{2}=0$. If it admits an extension to a pure (TLE)-structure, it is contained in Theorem 6.3. If not, then it is contained in Theorem 6.7. The condition that the Higgs field is not vanishing, excludes the 8th and 9th cases in Theorem 6.3 and the case $(6.28)=$ case (III) in Theorem 6.7, see Remarks 6.8(ii) and (iii). 
$(d)$ Part $(d)$ makes for such a $(T E)$-structure $\left(H^{[3]}, \nabla^{[3]}\right)$ the $(T E)$-structure $(\mathcal{O}(H), \nabla)=$ $\operatorname{pr}_{2}^{*}\left(\mathcal{O}\left(H^{[3]}\right), \nabla^{[3]}\right) \otimes \mathcal{E}^{\left(t_{1}+c_{1}\right) / z}$ explicit. The cooordinate $t$ and the matrix $A$ in Theorem 6.3 and in Remark 6.8(iv) become now $t_{2}$ and $A_{2}$. Here the matrices in the 6th case in Theorem 6.3 are not used, but the matrices in Remark 6.8(iv). The function $f$ in Remark 6.8(iv) is now specialized to $f=t_{2}^{k_{1} / 2}$ if $\alpha_{1}=\alpha_{2}\left(\Rightarrow\right.$ case (II) and (6.31)) and to $f=t_{2}^{k_{1}}$ if $\alpha_{1}-\alpha_{2} \in \mathbb{N}$ (case (I) and (6.30) or case (II) and (6.31)). The new matrix $B$ is $\left(-t_{1}-c_{1}\right) C_{1}$ plus the matrix $B$ in Theorem 6.3 and in Remark 6.8(iv).

In the normal forms in Remark 6.8(iv) we replaced $\alpha_{1}$ and $\alpha_{2}$ by $\rho^{(1)}$ and $\alpha_{4}$ as follows

$$
\rho^{(1)}:=\frac{\alpha_{1}+\alpha_{2}+1}{2}, \quad \alpha_{4}:= \begin{cases}\alpha_{1}-\alpha_{2}-1 \in \mathbb{N}_{0} & \text { in (6.30), } \\ \alpha_{2}-\alpha_{1}-1 \in \mathbb{Z}_{<0} & \text { in (6.31). }\end{cases}
$$

(b) Now part (b) follows from inspection of the normal forms in part $(d)$.

(a) Consider a $(T E)$-structure as in $(a)$. Choose coordinates $t=\left(t_{1}, t_{2}\right)$ on $\left(M, t^{0}\right)$ such that $\left(M, t^{0}\right)=\left(\mathbb{C}^{2}, 0\right)$ and the germ of the $F$-manifold is in a normal form in Theorem 2.2 (especially $\left.e=\partial_{1}\right)$ and the Euler field has the form $E=\left(t_{1}+c_{1}\right) \partial_{1}+g\left(t_{2}\right) \partial_{2}$ for some $c_{1} \in \mathbb{C}$ and some $g\left(t_{2}\right) \in \mathbb{C}\left\{t_{2}\right\}$.

Choose any $\mathbb{C}\{t, z\}$-basis $\underline{v}$ of $\mathcal{O}(H)_{0}$ and consider its matrices $A_{1}, A_{2}, B$ with $z \nabla_{\partial_{i}} \underline{v}=\underline{v} A_{i}$, $z^{2} \nabla_{\partial_{z}} \underline{v}=\underline{v} B$. Now $\partial_{1}=e$ implies $A_{1}^{(0)}=C_{1}$. We make a base change with the matrix $T \in \mathrm{GL}_{2}(\mathbb{C}\{t, z\})$ which is the unique solution of the differential equation

$$
\partial_{1} T=-\left(\sum_{k \geq 1} A_{1}^{(k)} z^{k-1}\right) T, \quad T\left(z, 0, t_{2}\right)=C_{1} .
$$

Then the matrices $\widetilde{A}_{1}, \widetilde{A}_{2}, \widetilde{B}$ of the new basis $\underline{\widetilde{v}}=\underline{v} T$ satisfy

$$
\widetilde{A}_{1}=C_{1}, \quad \partial_{1} \widetilde{A}_{2}=0, \quad \partial_{1} \widetilde{B}=-C_{1},
$$

because (3.10) for $i=1$ and (3.5) and (3.6) give

$$
\begin{aligned}
& 0=z \partial_{1} T+A_{1} T-T \widetilde{A}_{1}=C_{1} T-T \widetilde{A}_{1}=T\left(C_{1}-\widetilde{A}_{1}\right), \\
& 0=z \partial_{1} \widetilde{A}_{2}-z \partial_{2} \widetilde{A}_{1}+\left[\widetilde{A}_{1}, \widetilde{A}_{2}\right]=z \partial_{1} \widetilde{A}_{2}, \\
& 0=z \partial_{1} B-z^{2} \partial_{z} \widetilde{A}_{1}+z \widetilde{A}_{1}+\left[\widetilde{A}_{1}, \widetilde{B}\right]=z\left(\partial_{1} B+C_{1}\right) .
\end{aligned}
$$

In Lemmata 3.10(c) and 3.11 we considered the $(T E)$-structure $\left(\mathcal{O}\left(H^{[2]}\right), \nabla^{[2]}\right)=(\mathcal{O}(H), \nabla)$ $\otimes \mathcal{E}^{\rho^{(0)} / z}$ with trace free pole part. Here $\rho^{(0)}=-t_{1}-c_{1}$.

(8.2) shows that $\left(H^{[2]}, \nabla^{[2]}\right)$ is the pull back of its restriction $\left(H^{[3]}, \nabla^{[3]}\right)$ to $(\{0\} \times \mathbb{C}, 0) \subset$ $\left(\mathbb{C}^{2}, 0\right)$. This and $(\mathcal{O}(H), \nabla) \cong\left(\mathcal{O}\left(H^{[2]}\right), \nabla^{[2]}\right) \otimes \mathcal{E}^{-\rho^{(0)} / z}$ in Lemma 3.10(c) imply (8.1).

Corollary 8.3. The following table gives for each logarithmic rank 2 (TE)-structure over a point $t^{0}$ its unfoldings within the set of (TE)-structures in Theorem $8.1(d)$. Here the set $\left\{\alpha_{1}^{0}, \alpha_{2}^{0}\right\} \subset \mathbb{C}$ is the set of leading exponents in Theorem 4.20 of the logarithmic (TE)-structure over $t^{0}$. So, in the case $N^{\mathrm{mon}}=0, \alpha_{1}^{0}$ and $\alpha_{2}^{0} \in \mathbb{C}$ are arbitrary. In the case $N^{\mathrm{mon}} \neq 0$, they satisfy $\alpha_{1}^{0}-\alpha_{2}^{0} \in \mathbb{N}_{0}$. Two conditions are $c_{1}=0$ and $\rho^{(1)}=\frac{\alpha_{1}^{0}+\alpha_{2}^{0}}{2}$. The other conditions and the other invariants (though without their definition domains) are given in the table. All invariants 
in Theorem 8.1(d), which are not mentioned here, are (intended to be) arbitrary:

\begin{tabular}{l|l|l|l}
\hline \multicolumn{1}{c|}{ Generic type } & Invariants & $N^{\text {mon }}$ & Condition \\
\hline$($ Sem $): k_{2}>k_{1}$ & $k_{1}, k_{2}, \zeta$ & $=0$ & $\alpha_{1}^{0}-\alpha_{2}^{0}= \pm \frac{k_{1}-k_{2}}{k_{1}+k_{2}}$ \\
$($ Sem $): k_{2}=k_{1}$ & $k_{1}, k_{2}, \alpha_{3}$ & $=0$ & $\alpha_{1}^{0}-\alpha_{2}^{0}= \pm 2 \alpha_{3}$ \\
\hline$($ Bra $)$ & $k_{1}, k_{2}$ & $=0$ & $\alpha_{1}^{0}-\alpha_{2}^{0}= \pm \frac{-k_{2}}{k_{1}+k_{2}}$ \\
\hline$($ Reg $)$ & $k_{1}, \alpha_{4}$ & $=0$ & $\alpha_{1}^{0}-\alpha_{2}^{0}= \pm \alpha_{4}$ \\
\hline$($ Reg $)$ & $\widetilde{k}_{1}, \alpha_{4}$ & $\neq 0$ & $\alpha_{1}^{0}-\alpha_{2}^{0}=\left|\alpha_{4}\right|$ \\
\hline$($ Log $)$ & $k_{1}$ & $=0$ & $\alpha_{1}^{0}-\alpha_{2}^{0}= \pm 1$ \\
\hline
\end{tabular}

Proof. This follows from inspection of the cases in Theorem $8.1(d)$.

Remark 8.4. Beware of the following:

(i) In the generic case (Sem) with $k_{1}=k_{2}$ we have $\alpha_{3} \in \mathbb{R}_{\geq 0} \cup \mathbb{H}$. Here $\widetilde{\alpha}_{3}=-\alpha_{3}$ is excluded, as it gives an isomorphic unfolding.

(ii) In the generic cases (Reg) with $\alpha_{1}^{0}-\alpha_{2}^{0} \in \mathbb{C} \backslash\{0\}$ almost always $\alpha_{4}=\alpha_{1}^{0}-\alpha_{2}^{0}$ and $\widetilde{\alpha}_{4}=-\alpha_{4}$ give (for the same $k_{1} \in \mathbb{N}$ respectively $\widetilde{k}_{1} \in \mathbb{N}$ ) two different unfoldings. The only exception is the case $N^{\text {mon }}=0$ and $\alpha_{1}^{0}-\alpha_{2}^{0}= \pm 1$, as then $\alpha_{4}=-1$ is not allowed.

(iii) In the generic case ( $\log$ ), one has one unfolding (and not two unfoldings) for each $k_{1} \in \mathbb{N}$.

(iv) Unfoldings of generic type (Sem) with $k_{2}>k_{1}$ and of generic type (Bra) exist only if $\alpha_{1}^{0}-\alpha_{2}^{0} \in(-1,1) \cap \mathbb{Q}^{*}$ and $N^{\text {mon }}=0$.

\section{Theorem 8.5.}

(a) Any unfolding of a rank 2 (TE)-structure of type (Log) over a point is induced by one in the following subset of (TE)-structures in Theorem 8.1 $(d)$ :

\begin{tabular}{l|l}
\hline Generic type and invariants & \multicolumn{1}{c}{ Condition } \\
\hline$($ Sem $): k_{2}-k_{1}>0$ odd & $\operatorname{gcd}\left(k_{1}, k_{2}\right)=1$ \\
$($ Sem $): \quad k_{2}-k_{1} \in 2 \mathbb{N}, \zeta=0$ & $\operatorname{gcd}\left(k_{1}, k_{2}\right)=1$ \\
$($ Sem $): \quad k_{2}-k_{1} \in 2 \mathbb{N}, \zeta \neq 0$ & $\operatorname{gcd}\left(k_{1}, \frac{k_{1}+k_{2}}{2}\right)=1$ \\
$($ Sem $): \quad k_{2}=k_{1}$ & $k_{2}=k_{1}=1$ \\
\hline$($ Bra $)$ & $\operatorname{gcd}\left(k_{1}, k_{2}\right)=1$ \\
\hline$($ Reg $): \quad N^{\text {mon }}=0$ & $k_{1}=1$ \\
\hline$($ Reg $): \quad N^{\text {mon }} \neq 0$ & $\widetilde{k}_{1}=1$ \\
\hline$($ Log $): \quad N^{\text {mon }}=0$ & $k_{1}=1$ \\
\hline
\end{tabular}

(b) The inducing (TE)-structure is not unique only if the original (TE)-structure has the form $\varphi^{*}\left(\mathcal{O}\left(H^{[5]}\right), \nabla^{[5]}\right) \otimes \mathcal{E}^{-\rho^{(0)} / z}$, where $\left(H^{[5]}, \nabla^{[5]}\right)$ is a logarithmic $(T E)$-structure over a point $t^{[5]}$ and $\varphi:\left(M, t^{0}\right) \rightarrow\left\{t^{[5]}\right\}$ is the projection, and $\left(H^{[5]}, \nabla^{[5]}\right)$ is not one with $N^{\text {mon }} \neq 0$ and equal leading exponents $\alpha_{1}=\alpha_{2}$. Then the original (TE)-structure is of type (Log) everywhere with Higgs field endomorphisms $C_{X} \in \mathcal{O}_{\left(M, t^{0}\right)}$. id for any $X \in \mathcal{T}_{\left(M, t^{0}\right)}$.

(c) The (TE)-structures in the list in (a) are universal unfoldings of themselves.

The proof of Theorem 8.5 will be given in Section 8.3. 
Theorem 8.6. The set of rank 2 (TE)-structures with primitive (not just generically primitive) Higgs field over a germ $\left(M, t^{0}\right)$ of an F-manifold and with restriction of type (Log) over $t^{0}$ is (after the choice of suitable coordinates) the proper subset of those in the list (8.4) in Theorem 8.5 which satisfy $k_{1}=1$ respectively $\widetilde{k}_{1}=1$. In the cases (Reg) and (Log), it coincides with the list (8.4). In the cases (Sem) and (Bra), it is a proper subset.

Proof. The set of rank 2 (TE)-structures with primitive Higgs field over a germ $\left(M, t^{0}\right)$ of an $F$-manifold and with restriction of type $(\log )$ over $t^{0}$ consists by Theorem $8.1(a)+(d)$ of those $(T E)$-structures in Theorem $8.1(d)$ which satisfy $A_{2}\left(t_{2}=0\right) \notin \mathbb{C} \cdot C_{1}$. This holds if and only if $k_{1}=1$ respectively $\widetilde{k}_{1}=1\left(\widetilde{k}_{1}=1\right.$ if the generic type is (Reg) and $\left.N^{\text {mon }} \neq 0\right)$, and then $A_{2}\left(t_{2}=0\right) \in\left\{-\gamma C_{2},-\gamma D, C_{2}\right\}$. Obviously, this is a proper subset of those in table (8.4) in the generic cases (Sem) and (Bra), and it coincides with those in table (8.4) in the generic cases $(\operatorname{Reg})$ and $(\log )$.

\section{$8.2(T E)$-structures over given $F$-manifolds with Euler fields}

Remarks 8.7. For a given germ $\left(\left(M, t^{0}\right), o, e, E\right)$ of an $F$-manifold with Euler field, define

$$
\begin{aligned}
& B_{1}\left(\left(M, t^{0}\right), \circ, e, E\right):=\left\{(T E) \text {-structures over }\left(M, t^{0}\right)\right. \text { with generically primitive Higgs } \\
&\text { field, inducing the given } F \text {-manifold structure with Euler field }\}, \\
& B_{2}\left(\left(M, t^{0}\right), \circ, e, E\right):=\left\{(T E) \text {-structures in } B_{1} \text { which are in table }(8.4)\right\}, \\
& B_{3}\left(\left(M, t^{0}\right), \circ, e, E\right):=\left\{(T E) \text {-structures in } B_{1} \text { with primitive Higgs fields }\right\} .
\end{aligned}
$$

Now we can answer the questions, how big these sets are. Often we write $B_{j}$ instead of $B_{j}\left(\left(M, t^{0}\right), \circ, e, E\right)$, when the germ $\left(\left(M, t^{0}\right), \circ, e, E\right)$ is fixed.

(i) First we consider the cases when the germ $\left(\left(M, t^{0}\right), \circ, e, E\right)$ is regular. Compare Remark 2.6(ii) and Remark 3.17(iii). By Malgrange's unfolding result Theorem 3.16(c), any $(T E)$-structure over $\left(M, t^{0}\right)$ is the universal unfolding of its restriction over $t^{0}$, and it is its own universal unfolding. So then $B_{1}=B_{2}=B_{3}$, and the classification of the $(T E)$-structures over points in Section 4 determines this space $B_{1}$.

In the case of $A_{1}^{2}$ with $E=\left(u_{1}+c_{1}\right) e_{1}+\left(u_{2}+c_{2}\right) e_{2}$ with $c_{1} \neq c_{2}$, any $(T E)$-structure over $t^{0}$ is of type (Sem). Theorem 4.5 tells that then $B_{1}$ is connected and 4-dimensional. The parameters are the two regular singular exponents and two Stokes parameters.

In the case of $\mathcal{N}_{2}$ with $E=\left(t_{1}+c_{1}\right) \partial_{1}+\partial_{2}$, any $(T E)$-structure over $t^{0}$ is either of type (Bra) or of type (Reg). Then $B_{1}$ has one component for type (Bra) and countably many components for type (Reg).

The component for type (Bra) is connected and 3-dimensional. The parameters are given in Theorem 4.11, they are $\rho^{(1)}, \delta^{(1)}$ and $\operatorname{Eig}\left(M^{\text {mon }}\right)$ (here $\rho^{(0)}\left(t^{0}\right)=-c_{1}$ is fixed, and one eigenvalue and $\rho^{(1)}$ determine the other eigenvalue).

Corollary 4.18 gives the countably many components for type (Reg). One is 1-dimensional, the others are 2-dimensional.

(ii) Now we consider the cases when the germ $\left(\left(M, t^{0}\right), \circ, e, E\right)$ is not regular. Then $\left.E\right|_{t^{0}}=$ $c_{1} \partial_{1}$ for some $c_{1} \in \mathbb{C}$. If $(\mathcal{O}(H), \nabla)$ is a $(T E)$-structure in $B_{j}\left(\left(M, t^{0}\right), \circ, e, E\right)$, then $(\mathcal{O}(H), \nabla) \otimes \mathcal{E}^{-c_{1} / z}$ is a $(T E)$-structure in $B_{j}\left(\left(M, t^{0}\right), \circ, e, E-c_{1} \partial_{1}\right)$. Therefore we can and will restrict to the cases with $\left.E\right|_{t^{0}}=0$.

Theorem 8.1 $(d)$ gives the $(T E)$-structures in $B_{1}$, Theorem 8.5 $(a)$ gives the $(T E)$-structures in $B_{2}$, and Theorem 8.6 gives the $(T E)$-structures in $B_{3}$. For each germ $\left(\left(M, t^{0}\right), \circ, e, E\right)$ 
with $\left.E\right|_{t^{0}}=0$

$$
B_{1} \supset B_{2} \supset B_{3}
$$

In the cases $A_{1}^{2}$ and $I_{2}(m)$, the Euler field with $\left.E\right|_{t^{0}}=0$ is unique on $\left(M, t^{0}\right)$, therefore we do not write it down.

In the case of $I_{2}(m)$ with $m \in 2 \mathbb{N}$ (this includes the case $A_{1}^{2}=I_{2}(2)$ )

$$
\begin{aligned}
& B_{1}\left(I_{2}(m)\right) \cong \underset{\left(k_{1}, k_{2}\right) \in \mathbb{N}^{2}: k_{1}+k_{2}=m, k_{2} \geq k_{1}}{\bigcup^{\cdot}} \mathbb{C}^{2}, \\
& \bigcup_{2}\left(I_{2}(m)\right) \cong \underset{\left(k_{1}, k_{2}\right) \in \mathbb{N}^{2}: k_{1}+k_{2}=m, k_{2} \geq k_{1}, \operatorname{gcd}\left(k_{1}, m / 2\right)=1}{\mathbb{C}^{2},} \\
& B_{3}\left(I_{2}(m)\right) \cong \mathbb{C}^{2}, \quad \text { here }\left(k_{1}, k_{2}\right)=(1, m-1) .
\end{aligned}
$$

The 2 continuous parameters are the regular singular exponents of the $(T E)$-structures at generic points in $M$.

In the case of $I_{2}(m)$ with $m \geq 3$ odd,

$$
\begin{aligned}
& B_{1}\left(I_{2}(m)\right) \cong \underset{\left(k_{1}, k_{2}\right) \in \mathbb{N}^{2}: k_{1}+k_{2}=m, k_{2}>k_{1}}{\bigcup_{\left(k_{1}, k_{2}\right) \in \mathbb{N}^{2}: k_{1}+k_{2}=m, k_{2}>k_{1}, \operatorname{gcd}\left(k_{1}, k_{2}\right)=1}} \mathbb{C}, \\
& B_{2}\left(I_{2}(m)\right) \cong \underset{C}{\mathbb{C}, \quad \text { here }\left(k_{1}, k_{2}\right)=(1, m-1) .}
\end{aligned}
$$

For odd $m \geq 3$, the regular singular exponents of the $(T E)$-structures at generic points in $M$ coincide and give the continuous parameter.

Especially, for $m \in\{2,3\}$

$$
B_{1}\left(I_{2}(m)\right)=B_{2}\left(I_{2}(m)\right)=B_{3}\left(I_{2}(m)\right) \cong \begin{cases}\mathbb{C}^{2} & \text { if } m=2, \\ \mathbb{C} & \text { if } m=3 .\end{cases}
$$

The $F$-manifold $\mathcal{N}_{2}$ allows by Theorem 2.3 many nonisomorphic Euler fields with $\left.E\right|_{t^{0}}=0$, the cases $(2.5)-(2.7)$ with $c_{1}=0$.

The case (2.5), $E=t_{1} \partial_{1}$ : Here each $(T E)$-structure has generic type (Log) and semisimple monodromy. Here

$$
\begin{aligned}
& B_{1}\left(\mathcal{N}_{2}, E\right) \cong \bigcup_{k_{1} \in \mathbb{N}} \mathbb{C} \\
& B_{2}\left(\mathcal{N}_{2}, E\right)=B_{3}\left(\mathcal{N}_{2}, E\right) \cong \mathbb{C}, \quad \text { here } \quad k_{1}=1
\end{aligned}
$$

The continuous parameter is $\rho^{(1)}$ in Theorem 8.1(d) (iv) or, equivalently, one of the two residue eigenvalues (which are $\rho^{(1)} \pm \frac{1}{2}$ ).

The case (2.7), $E=t_{1} \partial_{1}+t_{2}^{r}\left(1+c_{3} t_{2}^{r-1}\right)$ for some $r \in \mathbb{Z}_{\geq 2}$ and some $c_{3} \in \mathbb{C}$ : Here each $(T E)$-structure has generic type (Reg) and satisfies $N^{\text {mon }} \neq 0$. Here

$$
B_{1}\left(\mathcal{N}_{2}, E\right) \begin{cases}=\varnothing, & \text { if } \quad c_{3} \in \mathbb{C}^{*} \\ \cong \mathbb{C} & \text { if } \quad c_{3}=0\end{cases}
$$




$$
B_{2}\left(\mathcal{N}_{2}, E\right)=B_{3}\left(\mathcal{N}_{2}, E\right)\left\{\begin{array}{lll}
=\varnothing, & \text { if } \quad c_{3} \in \mathbb{C}^{*} \quad \text { or } \quad r \geq 3, \\
=B_{1}\left(\mathcal{N}_{2}, E\right) & \text { if } \quad c_{3}=0 \quad \text { and } \quad r=2 .
\end{array}\right.
$$

So, $\left(\mathcal{N}_{2}, E\right)$ with $c_{3} \in \mathbb{C}^{*}$ does not allow $(T E)$-structures over it, and $\left(\mathcal{N}_{2}, E\right)$ with $c_{3}=0$ and $r \geq 3$ does not allow $(T E)$-structures over it with primitive Higgs field. If $B_{j}\left(\mathcal{N}_{2}, E\right)$ $\neq \varnothing$ then $B_{j}\left(\mathcal{N}_{2}, E\right) \cong \mathbb{C}$ and the continuous parameter is $\rho^{(1)}$ in Theorem 8.1(d) (iii).

The case (2.6), $E=t_{1} \partial_{1}+c_{2} t_{2} \partial_{2}$ for some $c_{2} \in \mathbb{C}^{*}$ : This is a rich case. Here we decompose $B_{j}=B_{j}\left(\mathcal{N}_{2}, E\right)$ as

$$
B_{j}=B_{j}^{(\mathrm{Reg}), 0} \dot{\cup} B_{j}^{(\mathrm{Reg}), \neq 0} \dot{\cup} B_{j}^{(\mathrm{Bra})},
$$

where the first set contains $(T E)$-structures of generic type (Reg) with $N^{\text {mon }}=0$, the second set contains (TE)-structures of generic type (Reg) with $N^{\text {mon }} \neq 0$, and the third set contains $(T E)$-structures of generic type (Bra). Then

$$
\begin{aligned}
& B_{1}^{(\operatorname{Reg}), 0} \cong \bigcup_{k_{1} \in \mathbb{N}} \mathbb{C}, \quad B_{2}^{(\operatorname{Reg}), 0}=B_{3}^{(\operatorname{Reg}), 0} \cong \mathbb{C}, \\
& B_{1}^{(\mathrm{Reg}), \neq 0}\left\{\begin{array}{lll}
=\varnothing & \text { if } & c_{2} \in \mathbb{C}^{*} \backslash \mathbb{Q}^{*}, \\
\cong \dot{U}_{\left(k_{1}, \alpha_{4}\right) \in \mathbb{N} \times \mathbb{Z}: k_{1} c_{2}=1+\alpha_{4}} \mathbb{C} & \text { if } & c_{2} \in \mathbb{Q}^{*},
\end{array}\right. \\
& B_{2}^{(\operatorname{Reg}), \neq 0}=B_{3}^{(\operatorname{Reg}), \neq 0}\left\{\begin{array}{lll}
=\varnothing & \text { if } & c_{2} \in \mathbb{C} \backslash \mathbb{Z}, \\
\cong \mathbb{C} & \text { if } & c_{2} \in \mathbb{Z} \backslash\{0\},
\end{array}\right. \\
& B_{1}^{(\mathrm{Bra})}=B_{2}^{(\mathrm{Bra})}=B_{3}^{(\mathrm{Bra})}=\varnothing \quad \text { if } \quad c_{2}^{-1} \in \mathbb{C}^{*} \backslash \mathbb{Z}_{\geq 2}, \\
& B_{1}^{(\mathrm{Bra})} \cong \dot{\bigcup}_{\left(k_{1}, k_{2}\right) \in \mathbb{N}^{2}: k_{1}+k_{2}=c_{2}^{-1}} \mathbb{C}, \\
& \left.B_{2}^{(\mathrm{Bra})} \cong \dot{\bigcup}_{\left(k_{1}, k_{2}\right) \in \mathbb{N}^{2}: k_{1}+k_{2}=c_{2}^{-1}, \operatorname{gcd}\left(k_{1}, k_{2}\right)=1} \mathbb{C},\right\} \quad \text { if } \quad c_{2}^{-1} \in \mathbb{Z}_{\geq 2} \text {. } \\
& B_{3}^{(\text {Bra })} \cong \mathbb{C}, \quad \text { here }\left(k_{1}, k_{2}\right)=\left(1, c_{2}^{-1}-1\right)
\end{aligned}
$$

\section{Remarks 8.8.}

(i) Theorem 8.1(d) $(i)$ tells how many $(T E)$-structures exist over the $F$-manifold with Euler field $I_{2}(m)$, such that the Higgs bundle is generically primitive and induces this $F$-manifold structure. There are $\left[\frac{m}{2}\right]$ many holomorphic families from the different choices of $\left(k_{1}, k_{2}\right)$ $\in \mathbb{N}^{2}$ with $k_{2} \geq k_{1}$ and $k_{1}+k_{2}=m$. They have 2 parameters if $m$ is even and 1 parameter if $m$ is odd, compare (8.5) and (8.6). For each $I_{2}(m)$, only one of these families consists of $(T E)$-structures with primitive Higgs fields.

(ii) Consider $m \geq 3$. Write $M=\mathbb{C}^{2}$ for the $F$-manifold $I_{2}(m)$ in Theorem 2.2 , and $M^{[\log ]}=$ $\mathbb{C} \times\{0\}$ for the subset of points where the multiplication is not semisimple. Over these points the restricted $(T E)$-structures are of type $(\log )$. We checked that there are $\left[\frac{m}{2}\right]$ many Stokes structures which give $(T E)$-structures on $M \backslash M^{[\log ]}$. Because of $(i)$, all these (TE)-structures extend holomorphically over $M^{[\log ]}$, and they give the $\left[\frac{m}{2}\right]$ holomorphic families of $(T E)$-structures on $I_{2}(m)$ in $(i)$.

(iii) Especially remarkable is the case $A_{1}^{2}=I_{2}(2)$. There Theorem $8.1(a)+(d)(i)$ implies directly that each holomorphic $(T E)$-structure over $A_{1}^{2}$ with generically primitive Higgs field has primitive Higgs field and is an elementary model (Definition 4.4), so it has trivial Stokes structure.

(iv) This result is related to much more general work in [3] and [22] on meromorphic connections over the $F$-manifold $A_{1}^{n}$ near points where some of the canonical coordinates coincide. Let 
us restrict to the special case of a neighborhood of a point where all canonical coordinates coincide. This generalizes the germ at 0 of $A_{1}^{2}$ to the germ at 0 of $A_{1}^{n}$.

[3, Theorem 1.1] and [22, Theorem 3] both give the triviality of the Stokes structure. Though their starting points are slightly restrictive. [3] starts in our notation from pure (TLE)-structures with primitive Higgs fields. The step before in the case of $A_{1}^{2}$, passing from a $(T E)$-structure over $A_{1}^{2}$ to a pure $(T L E)$-structure, is done essentially in our Theorem $6.2(a)(\mathrm{iii})$. Our argument for the triviality of the Stokes structure is then contained in the proof of Theorem 6.3.

[22] starts in our notation from $(T E)$-structures which are already formally isomorphic to sums $\bigoplus_{i=1}^{n} \mathcal{E}^{u_{i} / z} z^{\alpha_{i}}$. Then it is shown that they are also holomorphically isomorphic to such sums. In this special case, Corollary 5.7 in [7] give this implication, too.

(v) In $(i i)$ we stated that in the case of $I_{2}(m)$ with $m \geq 3$, each $(T E)$-structure on $M \backslash M^{[\log ]}$ with primitive Higgs field extends holomorphically to $M$. In the case of $\mathcal{N}_{2}$ this does not hold in general. For example, start with the flat rank 2 bundle $H^{\prime} \rightarrow \mathbb{C}^{*} \times M$, where $M=\mathbb{C}^{2}$ (with coordinates $t=\left(t_{1}, t_{2}\right)$ ) with semisimple monodromy with two different eigenvalues $\lambda_{1}$ and $\lambda_{2}$. Choose $\alpha_{1}, \alpha_{2} \in \mathbb{C}$ with $\mathrm{e}^{-2 \pi \mathrm{i} \alpha_{j}}=\lambda_{j}$. Let $s_{j} \in C^{\alpha_{j}}$ be generating elementary sections. Define the new basis

$$
\underline{v}=\left(v_{1}, v_{2}\right)=\left(\mathrm{e}^{t_{1} / z}\left(s_{1}+\mathrm{e}^{-1 / t_{2}} s_{2}\right), \mathrm{e}^{t_{1} / z}\left(z s_{2}\right)\right)
$$

on $\left.H^{\prime}\right|_{M^{\prime}}$, where $M^{\prime}:=M \backslash \mathbb{C} \times\{0\}$. Then

$$
\begin{aligned}
& z \nabla_{\partial_{1} \underline{v}}=\underline{v} \cdot C_{1}, \\
& z \nabla_{\partial_{2} \underline{v}}=\underline{v} \cdot t_{2}^{-2} \mathrm{e}^{-1 / t_{2}} C_{2}, \\
& z^{2} \nabla_{\partial_{z} \underline{v}}=\underline{v} \cdot\left(-t_{1} C_{1}+\left(\alpha_{2}-\alpha_{1}\right) \mathrm{e}^{-1 / t_{2}} C_{2}+z\left(\begin{array}{cc}
\alpha_{1} & 0 \\
0 & \alpha_{2}+1
\end{array}\right)\right) .
\end{aligned}
$$

So, we obtain a regular singular $(T E)$-structure on $M^{\prime}$ with primitive Higgs field. The $F$-manifold structure on $M^{\prime}$ is given by $e=\partial_{1}$ and $\partial_{2} \circ \partial_{2}=0$, so it is $\mathcal{N}_{2}$, and the Euler field is $E=t_{1} \partial_{1}+\left(\alpha_{1}-\alpha_{2}\right) t_{2}^{2} \partial_{2}$. $F$-manifold and Euler field extend from $M^{\prime}$ to $M$, but not the $(T E)$-structure.

\subsection{Proof of Theorem 8.5}

(a) Let $\left(H \rightarrow \mathbb{C} \times\left(M, t^{0}\right), \nabla\right)$ be an unfolding of a $(T E)$-structure of type (Log) over $t^{0}$. The $(T E)$-structure $\left(H^{[2]} \rightarrow \mathbb{C} \times\left(M, t^{0}\right), \nabla^{[2]}\right)$ in Lemma $3.10(c)$ with $\left(\mathcal{O}\left(H^{[2]}\right), \nabla^{[2]}\right)=(\mathcal{O}(H), \nabla) \otimes$ $\mathcal{E}^{-\rho^{(0)} / z}$ has trace free pole part. Lemma $3.10(d)$ and $(e)$ apply. Because of them, it is sufficient to prove that the $(T E)$-structure $\left(H^{[2]}, \nabla^{[2]}\right)$ is induced by a $(T E)$-structure $\left(H^{[3]} \rightarrow \mathbb{C} \times\right.$ $\left.\left(M^{[3]}, t^{[3]}\right), \nabla^{[3]}\right)$ over $\left(M^{[3]}, t^{[3]}\right)=(\mathbb{C}, 0)$ via a map $\varphi:\left(M, t^{0}\right) \rightarrow\left(M^{[3]}, t^{[3]}\right)$, where the $(T E)$ structure $\left(H^{[3]}, \nabla^{[3]}\right)$ is one of the $(T E)$-structures in the 1 st to 7 th cases in Theorem 6.3 or one of the (TE)-structures in the cases (I) or (II) in Theorem 6.7 with invariants as in table (8.4). Then the $(T E)$-structure $\left(H^{[4]}, \nabla^{[4]}\right)$ which is constructed in Lemma $3.10(d)$ from $\left(H^{[3]}, \nabla^{[3]}\right)$ is one of the $(T E)$-structures in Theorem $8.1(d)$ with invariants as in table (8.4), and it induces by Lemma $3.10(e)$ the $(T E)$-structure $(H, \nabla)$.

From now on we suppose $\rho^{(0)}=0$, so $(H, \nabla)=\left(H^{[2]}, \nabla^{[2]}\right)$. We consider the invariants $\delta^{(0)}, \delta^{(1)} \in \mathcal{O}_{M, t^{0}}$ and $\mathcal{U}$ and the four possible generic types (Sem), (Bra), (Reg) and (Log), which are defined by the following table, analogously to Definition 6.1,

\begin{tabular}{c|c|c|c}
\hline$($ Sem $)$ & $($ Bra $)$ & $($ Reg $)$ & $(\log )$ \\
\hline$\delta^{(0)} \neq 0$ & $\delta^{(0)}=0, \delta^{(1)} \neq 0$ & $\delta^{(0)}=\delta^{(1)}=0, \mathcal{U} \neq 0$ & $\mathcal{U}=0$ \\
\hline
\end{tabular}

First we treat the generic types (Reg) and ( Log), then the generic type (Sem) and (Bra). 
Generic types $(R e g)$ and $(\log )$. Then the $(T E)$-structure $(H, \nabla)$ is regular singular. We can use the results in Section 7 (which built on Theorems 6.3 and 6.7). Choose a marking for the (TE)structure $(H, \nabla)$. Then by Remark $7.5(i)$, there is a unique map $\varphi:\left(M, t^{0}\right) \rightarrow M^{\left(H^{\text {ref, } \infty}, M^{\text {ref }}\right) \text {,reg }}$ which maps $t \in M$ to the point in the moduli space over which one has up to isomorphism the same marked (TE)-structure as over $t$. The map $\varphi$ is holomorphic. By Remark $7.5(i)+(i i)$ it maps $\left(M, t^{0}\right)$ to one projective curve which is isomorphic to $M^{(3), 0, \alpha_{1}, \alpha_{2}}$ or $M^{(3), \neq 0, \alpha_{1}, \alpha_{2}}$ or

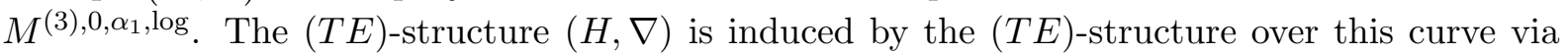
the map $\varphi$. The point $t^{0}$ is mapped to 0 or $\infty$ in the cases $M^{(3), 0, \alpha_{1}, \alpha_{2}}$ or $M^{(3), \neq 0, \alpha_{1}, \alpha_{2}}$ (not 0 in the case $\left.M^{(3), \neq 0, \alpha_{1}, \alpha_{1}}\right)$ as the (TE)-structure over $t^{0}$ is logarithmic. The germs at 0 and $\infty$ in $M^{(3), 0, \alpha_{1}, \alpha_{2}}$ and $M^{(3), \neq 0, \alpha_{1}, \alpha_{2}}$ (not 0 in the case $M^{(3), \neq 0, \alpha_{1}, \alpha_{1}}$ ) and the germ at any point $t_{2}^{(3)}$ in $M^{(3), 0, \alpha_{1}, \log }$ are contained in table (8.4). This shows Theorem 8.5 for the generic cases (Reg) and $(\log )$.

Generic types (Sem) and (Bra). We choose a (connected and sufficiently small) representative $M$ of the germ $\left(M, t^{0}\right)$, and we choose on it coordinates $t=\left(t_{1}, \ldots, t_{m}\right)$ (with $m=\operatorname{dim} M$ ) with $t^{0}=0$. We denote by $M^{[\log ]}$ the analytic hypersurface

$$
M^{[\log ]}:= \begin{cases}\left(\delta^{(0)}\right)^{-1}(0), & \text { if the generic type is (Sem) } \\ \left(\delta^{(1)}\right)^{-1}(0), & \text { if the generic type is (Bra) }\end{cases}
$$

It contains $t^{0}$. Choose a disk $\Delta \subset M$ through $t^{0}$ with $\Delta \backslash\left\{t^{0}\right\} \subset M \backslash M^{[\log ]}$. The restricted $(T E)$-structure $\left.(H, \nabla)\right|_{\mathbb{C} \times\left(\Delta, t^{0}\right)}$ has the same generic type as the $(T E)$-structure $(H, \nabla)$. The restricted $(T E)$-structure $\left.(H, \nabla)\right|_{\mathbb{C} \times\left(\Delta, t^{0}\right)}$ is isomorphic to a $(T E)$-structure in the cases $1,2,3$ or 4 in Theorem 6.3 .

The parameters of the restricted $(T E)$-structure $\left.(H, \nabla)\right|_{\mathbb{C} \times\left(\Delta, t^{0}\right)}$ are given in the following table:

\begin{tabular}{c|c}
\hline Generic type & Parameters \\
\hline$($ Sem $)$ & $k_{1}, k_{2} \in \mathbb{N}$ with $k_{2} \geq k_{1}, \rho^{(1)} \in \mathbb{C}$, \\
& $\begin{cases}\zeta \in \mathbb{C} & \text { if } k_{2}-k_{1} \in 2 \mathbb{N}, \\
\alpha_{3} \in \mathbb{R} \geq 0 \cup \mathbb{H} & \text { if } k_{1}=k_{2}\end{cases}$ \\
$($ Bra $)$ & $k_{1}, k_{2} \in \mathbb{N}, \rho^{(1)} \in \mathbb{C}$
\end{tabular}

There is a unique pair $\left(k_{1}^{0}, k_{2}^{0}\right) \in \mathbb{N}^{2}$ with $\left(k_{1}, k_{2}\right) \in \mathbb{Q}_{>0} \cdot\left(k_{1}^{0}, k_{2}^{0}\right)$ and with the conditions in table (8.7),

\begin{tabular}{l|l}
\hline Generic type and invariants & \multicolumn{1}{|c}{ Conditions } \\
\hline$($ Sem $): k_{2}-k_{1}>0$ odd & $\operatorname{gcd}\left(k_{1}^{0}, k_{2}^{0}\right)=1$ \\
$($ Sem $): k_{2}-k_{1} \in 2 \mathbb{N}, \zeta=0$ & $\operatorname{gcd}\left(k_{1}^{0}, k_{2}^{0}\right)=1$ \\
$($ Sem $): k_{2}-k_{1} \in 2 \mathbb{N}, \zeta \neq 0$ & $k_{2}^{0}-k_{1}^{0} \in 2 \mathbb{N}, \operatorname{gcd}\left(k_{1}^{0}, \frac{k_{1}^{0}+k_{2}^{0}}{2}\right)=1$ \\
$(\mathrm{Sem}): k_{2}=k_{1}$ & $k_{2}^{0}=k_{1}^{0}=1$ \\
\hline$($ Bra $)$ & $\operatorname{gcd}\left(k_{1}^{0}, k_{2}^{0}\right)=1$ \\
\hline
\end{tabular}

In fact, it is the pair $\left(k_{1}^{0}, k_{2}^{0}\right) \in \mathbb{N}^{2}$ of minimal numbers which satisfies

$$
\left(k_{1}, k_{2}\right) \in \mathbb{N} \cdot\left(k_{1}^{0}, k_{2}^{0}\right)
$$

and which satisfies in the case (Sem) with $k_{2}-k_{1} \in 2 \mathbb{N}$ and $\zeta \neq 0$ additionally $k_{2}^{0}-k_{1}^{0} \in 2 \mathbb{N}$. 
We denote by $\left(H^{[3]} \rightarrow \mathbb{C} \times\left(M^{[3]}, t^{[3]}\right), \nabla^{[3]}\right)$ the $(T E)$-structure over $\left(M^{[3]}, t^{[3]}\right)=(\mathbb{C}, 0)$ which has $\left(k_{1}^{0}, k_{2}^{0}\right)$ instead of $\left(k_{1}, k_{2}\right)$, but which has the same other parameters as the restricted $(T E)$-structure $\left.(H, \nabla)\right|_{\mathbb{C} \times\left(\Delta, t^{0}\right)}$.

We have seen in Remarks 6.5(ii) and (iii) that the restricted $(T E)$-structure $\left.(H, \nabla)\right|_{\mathbb{C} \times\left(\Delta, t^{0}\right)}$ is induced by the $(T E)$-structure $\left(H^{[3]}, \nabla^{[3]}\right)$ via the branched covering $\varphi^{\Delta}:\left(\Delta, t^{0}\right) \rightarrow\left(M^{[3]}, t^{[3]}\right)$ with $\varphi^{\Delta}(\tau)=\tau^{k_{1} / k_{1}^{0}}$. Here $\tau$ denotes that coordinate on $\Delta$ with which $\left.(H, \nabla)\right|_{\mathbb{C} \times\left(\Delta, t^{0}\right)}$ can be brought to a normal form in the cases $1,2,3$ and 4 in Theorem 6.3.

It rests to extend $\varphi^{\Delta}$ to a map $\varphi: M \rightarrow M^{[3]}$ such that $(H, \nabla)$ is induced by $\left(H^{[3]}, \nabla^{[3]}\right)$ via this map $\varphi$.

Claim 8.9. There exists a unique holomorphic function $\varphi \in \mathcal{O}_{M}$ with

$$
\begin{aligned}
& \left.\varphi\right|_{\Delta}=\varphi^{\Delta}, \\
& \delta^{(0)}=-\varphi^{k_{1}^{0}+k_{2}^{0}} \quad \text { if the generic type is }(\text { Sem }), \\
& \delta^{(1)}=\frac{k_{2}^{0}}{k_{1}^{0}+k_{2}^{0}} \cdot \varphi^{k_{1}^{0}+k_{2}^{0}} \quad \text { if the generic type is (Bra). }
\end{aligned}
$$

Proof. Choose any point $t^{[1]} \in M^{[\log ]}$ and any disk $\Delta^{[1]}$ through $t^{[1]}$ with $\Delta^{[1]} \backslash\left\{t^{[1]}\right\} \subset$ $M \backslash M^{[\log ]}$. In order to show the existence of a function $\varphi \in \mathcal{O}_{M}$ with (8.9) respectively (8.10), it is sufficient to show that $\left.\delta^{(0)}\right|_{\Delta^{[1]}}$ respectively $\left.\delta^{(1)}\right|_{\Delta^{[1]}}$ has at $t^{[1]}$ a zero of an order which is a multiple of $k_{1}^{0}+k_{2}^{0}$.

The restricted $(T E)$-structure $\left.(H, \nabla)\right|_{\mathbb{C} \times\left(\Delta^{[1]}, t^{[1]}\right)}$ has the same generic type as $(H, \nabla)$ and is isomorphic to a (TE)-structure in the cases $1,2,3$ or 4 in Theorem 6.3. Its invariants $k_{1}$ and $k_{2}$ are here called $k_{1}^{[1]}$ and $k_{2}^{[1]}$, in order to distinguish them from the invariants of $\left.(H, \nabla)\right|_{\left(\Delta, t^{0}\right)}$. We want to show

$$
\left(k_{1}^{[1]}, k_{2}^{[2]}\right) \in \mathbb{N} \cdot\left(k_{1}^{0}, k_{2}^{0}\right) .
$$

We did not say much about the Stokes structure. Here we need the following properties of it, if the generic type is (Sem):

$$
\begin{aligned}
k_{2} & =k_{1} \\
& \left.\stackrel{(1)}{\Longleftrightarrow}(H, \nabla)\right|_{\mathbb{C} \times\left\{t^{[2]}\right\}} \text { has trivial Stokes structure for } t^{[2]} \in \Delta \backslash\left\{t^{0}\right\} \\
& \left.\stackrel{(2)}{\Longleftrightarrow}(H, \nabla)\right|_{\mathbb{C} \times\left\{t^{[2]}\right\}} \text { has trivial Stokes structure for } t^{[2]} \in \Delta^{[1]} \backslash\left\{t^{[1]}\right\} \\
& \stackrel{(3)}{\Longleftrightarrow} k_{2}^{[1]}=k_{1}^{[1]} .
\end{aligned}
$$

$\stackrel{(1)}{\Longrightarrow}$ and $\stackrel{(3)}{\Longleftarrow}$ are obvious from the normal form in the 3 rd case in Theorem 6.3 . It is not hard to see that the normal forms for fixed $t \in \mathbb{C}^{*}$ in the 1 st and 2 nd case in Theorem 6.3 are not holomorphically isomorphic to an elementary model in Definition 4.4 (see also Remark 8.8(ii)). This shows $\stackrel{(1)}{=}$ and $\stackrel{(3)}{\Longleftrightarrow}$. The equivalence $\stackrel{(2)}{\Longleftrightarrow}$ is a consequence of the invariance of the Stokes structure within isomonodromic deformations.

In the generic type (Sem) with $k_{1}=k_{2}$ we have also $k_{2}^{[1]}=k_{1}^{[1]}$ and $k_{2}^{0}=k_{1}^{0}=1$, and thus especially (8.11).

Now consider the cases with $k_{2}>k_{1}$. This comprises the generic type (Bra) and gives in the generic type (Sem) also $k_{2}^{0}>k_{1}^{0}$ and $k_{2}^{[1]}>k_{1}^{[1]}$. So $\left.(H, \nabla)\right|_{\mathbb{C} \times\left(\Delta^{[1]}, t^{[1]}\right)}$ is in the 1 st, 2 nd or 4 th case in Theorem 6.3. The number $b_{3}^{(1)}$ in Theorem 6.3 is uniquely determined by the properties 
$\left.b_{3}^{(1)} \in \mathbb{Q} \cap\right] \frac{-1}{2}, 0\left[\right.$ and $\operatorname{Eig}\left(M^{\text {mon }}\right)=\left\{\exp \left(-2 \pi \mathrm{i}\left(\rho^{(1)} \pm b_{3}^{(1)}\right)\right)\right\}$ (see Remark 6.4(i) for the second property). Therefore

$$
\begin{array}{ll}
\frac{k_{1}^{0}-k_{2}^{0}}{2\left(k_{1}^{0}+k_{2}^{0}\right)}=\frac{k_{1}-k_{2}}{2\left(k_{1}+k_{2}\right)}=b_{3}^{(1)}=\frac{k_{1}^{[1]}-k_{2}^{[1]}}{2\left(k_{1}^{[1]}+k_{2}^{[1]}\right)} \quad \text { in the case (Sem), } \\
\frac{-k_{2}^{0}}{2\left(k_{1}^{0}+k_{2}^{0}\right)}=\frac{-k_{2}}{2\left(k_{1}+k_{2}\right)}=b_{3}^{(1)}=\frac{-k_{2}^{[1]}}{2\left(k_{1}^{[1]}+k_{2}^{[1]}\right)} \quad \text { in the case (Bra). }
\end{array}
$$

This implies $\left(k_{1}^{[1]}, k_{2}^{[1]}\right) \in \mathbb{Q}_{>0} \cdot\left(k_{1}^{0}, k_{2}^{0}\right)$. In the cases with $\operatorname{gcd}\left(k_{1}^{0}, k_{2}^{0}\right)=1$ (8.11) follows.

If $\operatorname{gcd}\left(k_{1}^{0}, k_{2}^{0}\right) \neq 1$, then the generic type is $(\mathrm{Sem}), k_{2}-k_{1} \in 2 \mathbb{N}, k_{2}^{0}-k_{1}^{0} \in 2 \mathbb{N}$, and the invariant $\zeta$ of $\left.(H, \nabla)\right|_{\mathbb{C} \times\left(\Delta, t^{0}\right)}$ is $\zeta \neq 0$. However, then the regular singular exponents $\alpha_{1}$ and $\alpha_{2}$ of the restriction of the $(T E)$-structure $(H, \nabla)$ over points in $M \backslash M^{[\log ]}$ are invariants of the $(T E)$-structure $(H, \nabla)$. By $(6.11)$ and (8.12) also $\zeta$ is an invariant of the $(T E)$-structure $(H, \nabla)$. Now $\zeta \neq 0$ implies $k_{2}^{[1]}-k_{1}^{[1]} \in 2 \mathbb{N}$. Again (8.11) follows.

Equations (6.8) and (6.12) imply that $\left.\delta^{(0)}\right|_{\Delta^{[1]}}$ respectively $\left.\delta^{(1)}\right|_{\Delta^{[1]}}$ has at $t^{[1]}$ a zero of an order which is a multiple of $k_{1}^{0}+k_{2}^{0}$. Therefore a function $\varphi \in \mathcal{O}_{M}$ with (8.9) respectively (8.10) exists.

Equations $(6.8)$ and $(6.12)$ for $\left.(H, \nabla)\right|_{\mathbb{C} \times\left(\Delta, t^{0}\right)}$ tell

$$
\begin{aligned}
& \left.\delta^{(0)}\right|_{\Delta}=-\tau^{k_{1}+k_{2}}=-\left(\tau^{k_{1} / k_{1}^{0}}\right)^{k_{1}^{0}+k_{2}^{0}}=-\left(\varphi^{\Delta}\right)^{k_{1}^{0}+k_{2}^{0}} \quad \text { in the case (Sem), } \\
& \left.\delta^{(1)}\right|_{\Delta}=\frac{k_{2}}{k_{1}+k_{2}} \tau^{k_{1}+k_{2}}=\frac{k_{2}^{0}}{k_{1}^{0}+k_{2}^{0}}\left(\tau^{k_{1} / k_{1}^{0}}\right)^{k_{1}^{0}+k_{2}^{0}}=\frac{k_{2}^{0}}{k_{1}^{0}+k_{2}^{0}}\left(\varphi^{\Delta}\right)^{k_{1}^{0}+k_{2}^{0}} \quad \text { in the case (Bra). }
\end{aligned}
$$

Therefore a function $\varphi$ as in the claim exists and is unique.

Now compare the $(T E)$-structures $(H, \nabla)$ and $\varphi^{*}\left(H^{[3]}, \nabla^{[3]}\right)$ over $M$. Both extend to pure $(T L E)$-structures. For $\varphi^{*}\left(H^{[3]}, \nabla^{[3]}\right)$, one uses the pull back $\varphi^{*}\left(\underline{v}^{[3]}\right)$ of the basis $\underline{v}^{[3]}$ which gives for $\left(H^{[3]}, \nabla^{[3]}\right)$ the Birkhoff normal form in Theorem 6.3. For $(H, \nabla)$ one starts with the analogous basis $\underline{v}^{\Delta}$ for $\left.H\right|_{\mathbb{C} \times \Delta}$ which gives for $\left.(H, \nabla)\right|_{\mathbb{C} \times \Delta}$ the Birkhoff normal form in Theorem 6.3. It has a unique extension $\underline{v}$ to $\mathbb{C} \times M$ which still yields a Birkhoff normal form. Compare Remark 3.19(ii) for this.

Remarks 6.5(ii) and (iii) (or simply the Birkhoff normal forms in Theorem 6.3) tell that the $\left.\operatorname{map}\left(\varphi^{*} \underline{v}^{[3]}\right)\right|_{\mathbb{C} \times \Delta} \mapsto \underline{v}^{\Delta}=\left.\underline{v}\right|_{\mathbb{C} \times \Delta}$ is an isomorphism of pure $(T L E)$-structures.

Now consider a point $t^{[2]} \in \Delta \backslash\left\{t^{0}\right\}$ and its image $t^{[4]}:=\varphi\left(t^{[2]}\right) \in M^{[3]} \backslash\left\{t^{[3]}\right\}=\mathbb{C} \backslash\{0\}$. Over the germ $\left(M^{[3]}, t^{[4]}\right)$, the $(T E)$-structure $\left(H^{[3]}, \nabla^{[3]}\right)$ is the part with trace free pole part of a universal unfolding of $\left.\left(H^{[3]}, \nabla^{[3]}\right)\right|_{\mathbb{C} \times\left\{t^{[4]}\right\}}$. Therefore in a neighborhood $U \subset M$ of $t^{[2]}$, the $(T E)$-structure $\left.(H, \nabla)\right|_{\mathbb{C} \times U}$ is induced by $\left.\left(H^{[3]}, \nabla^{[3]}\right)\right|_{\mathbb{C} \times\left(M^{[3]}, t^{[4]}\right)}$ via a map $\widetilde{\varphi}: U \rightarrow M^{[3]}$. We can choose it such that

$$
\left.\widetilde{\varphi}\right|_{\Delta}=\varphi^{\Delta} \text {. }
$$

Equations (6.8) and (6.12) tell

$$
\begin{aligned}
& \left.\delta^{(0)}\right|_{U}=-(\widetilde{\varphi})^{k_{1}^{0}+k_{2}^{0}} \quad \text { in the case (Sem), } \\
& \left.\delta^{(1)}\right|_{U}=\frac{k_{2}^{0}}{k_{1}^{0}+k_{2}^{0}}(\widetilde{\varphi})^{k_{1}^{0}+k_{2}^{0}} \quad \text { in the case (Bra). }
\end{aligned}
$$

Equations (8.13)-(8.15) and Claim 8.9 imply $\widetilde{\varphi}=\left.\varphi\right|_{U}$. Therefore the matrices in Birkhoff normal form for the basis $\underline{v}$ of $(H, \nabla)$ coincide on $\mathbb{C} \times U$ with the matrices in Birkhoff normal form for 
the basis $\varphi^{*}\left(\underline{v}^{[3]}\right)$ of $\varphi^{*}\left(H^{[3]}, \nabla^{[3]}\right)$. As all matrices are holomorphic on $\mathbb{C} \times M$, they coincide pairwise on $\mathbb{C} \times M$. Therefore the pure $(T L E)$-structure $(H, \nabla)$ with basis $\underline{v}$ is isomorphic to the pure $(T L E)$-structure $\varphi^{*}\left(H^{[3]}, \nabla^{[3]}\right)$ with basis $\varphi^{*}\left(\underline{v}^{[3]}\right)$. This finishes the proof of part $(a)$ of Theorem 8.5.

(b) If the original $(T E)$-structure $(H, \nabla)$ has the form $\varphi^{*}\left(\mathcal{O}\left(H^{[5]}\right), \nabla^{[5]}\right) \otimes \mathcal{E}^{-\rho^{(0)} / z}$ then the $(T E)$-structure $\left(H^{[2]}, \nabla^{[2]}\right)$ with trace free pole part which was associated to $(H, \nabla)$ at the beginning of the proof of part $(a)$, has the form $\varphi^{*}\left(\mathcal{O}\left(H^{[5]}\right), \nabla^{[5]}\right)$.

Then any $(T E)$-structure $\left(H^{[3]}, \nabla^{[3]}\right)$ over $\left(M^{[3]}, t^{[3]}\right)=(\mathbb{C}, 0)$ works, whose restriction over $t^{[3]}$ is the given logarithmic $(T E)$-structure $\left(H^{[5]}, \nabla^{[5]}\right)$.

In the cases with $N^{\text {mon }}=0$, table (8.4) offers one of generic type (Sem) with $k_{1}=k_{2}=1$ (and some with $k_{2}>k_{1}$ if $\alpha_{1}-\alpha_{2} \in \mathbb{Q} \cap(-1,1)$ ) and one or two of generic type (Reg), see table (8.3). In the cases with $N^{\text {mon }} \neq 0$, table (8.4) offers two of generic type (Reg) if the leading exponents $\alpha_{1}$ and $\alpha_{2}$ satisfy $\alpha_{1}-\alpha_{2} \in \mathbb{N}$, and one if they satisfy $\alpha_{1}=\alpha_{2}$, compare also Figures 7.4 and 7.5 in Theorem 7.4(b). Therefore the inducing (TE)-structure in table (8.4) is not unique except for the case $N^{\text {mon }} \neq 0$ and $\alpha_{1}=\alpha_{2}$, if the original (TE)-structure has the form $\varphi^{*}\left(\mathcal{O}\left(H^{[5]}\right), \nabla^{[5]}\right) \otimes \mathcal{E}^{-\rho^{(0)} / z}$.

In the other cases, the proof of part $(a)$ shows the uniqueness of the $(T E)$-structure $\left(H^{[3]}, \nabla^{[3]}\right)$. The uniqueness of $\left(H^{[3]}, \nabla^{[3]}\right)$ gives also the uniqueness of $\left(H^{[4]}, \nabla^{[4]}\right)$ in the first paragraph of the proof of part $(a)$.

(c) This follows from the uniqueness in part $(b)$.

\section{A family of rank $3(T E)$-structures with a functional parameter}

M. Saito presents in [23] a family of Gauss-Manin connections with a functional parameter. In the arXiv paper [23], the bundle has rank $n$, but in a preliminary version it has rank 3 and is more transparent.

Here we translate the rank 3 example by a Fourier-Laplace transformation into a family of $(T E)$-structures with primitive Higgs fields over a fixed 3-dimensional globally irreducible $F$ manifold with an Euler field, such that the $F$-manifold with Euler field is nowhere regular. The family of $(T E)$-structures has a functional parameter $h\left(t_{2}\right) \in \mathbb{C}\left\{t_{2}\right\}$.

In the following, we write down a $(T E)$-structure of rank 3 on a manifold $M=\mathbb{C}^{3}$ with coordinates $t_{1}, t_{2}, t_{3}$. The restriction to $\left\{t \in \mathbb{C}^{3} \mid t_{1}=0\right\}=\{0\} \times \mathbb{C}^{2}$ is a FL-transformation of Saito's example. The parameter $t_{1}$ and this $F$-manifold are not considered in [23]. There the base space has only the two parameters $t_{2}$ and $t_{3}$. Choose an arbitrary function $h\left(t_{2}\right) \in \mathbb{C}\left\{t_{2}\right\}$ with $h^{\prime \prime}(0) \neq 0$.

Let $H^{\prime} \rightarrow \mathbb{C}^{*} \times M$ be a holomorphic vector bundle with flat connection with trivial monodromy and basis of global flat sections $s_{1}, s_{2}, s_{3}$. Define an extension to a vector bundle $H \rightarrow \mathbb{C} \times M$ using the following holomorphic sections of $H^{\prime}$, which also form a basis of sections of $H^{\prime}$ :

$$
\begin{aligned}
& v_{1}:=\mathrm{e}^{t_{1} / z} \cdot\left(z s_{1}+t_{2} \cdot z s_{2}+h\left(t_{2}\right) \cdot z s_{3}+t_{3} \cdot z^{2} s_{3}\right), \\
& v_{2}:=\mathrm{e}^{t_{1} / z} \cdot\left(z^{2} s_{2}+h^{\prime}\left(t_{2}\right) \cdot z^{2} s_{3}\right), \quad v_{3}:=\mathrm{e}^{t_{1} / z} \cdot z^{3} s_{3} .
\end{aligned}
$$

Denote $\underline{v}:=\left(v_{1}, v_{2}, v_{3}\right)$. Denote $\partial_{t_{j}}:=\partial_{j}$. Then

$$
\begin{aligned}
& z \nabla_{\partial_{1} \underline{v}}=\underline{v} \cdot \mathbf{1}_{3}, \\
& z \nabla_{\partial_{2}} \underline{v}=\underline{v} \cdot\left(\begin{array}{ccc}
0 & 0 & 0 \\
1 & 0 & 0 \\
0 & h^{\prime \prime}\left(t_{2}\right) & 0
\end{array}\right),
\end{aligned}
$$




$$
\begin{aligned}
& z \nabla_{\partial_{3} \underline{v}}=\underline{v} \cdot\left(\begin{array}{ccc}
0 & 0 & 0 \\
0 & 0 & 0 \\
1 & 0 & 0
\end{array}\right), \\
& z^{2} \partial_{z} \underline{v}=\underline{v} \cdot\left(-t_{1} \cdot \mathbf{1}_{3}+\left(\begin{array}{ccc}
0 & 0 & 0 \\
0 & 0 & 0 \\
t_{3} & 0 & 0
\end{array}\right)\right)+z \cdot \underline{v} \cdot\left(\begin{array}{ccc}
1 & 0 & 0 \\
0 & 2 & 0 \\
0 & 0 & 3
\end{array}\right) .
\end{aligned}
$$

Write $\underline{\partial}:=\left(\partial_{1}, \partial_{2}, \partial_{3}\right)$. The pole parts give the multiplication $\circ$ on the $F$-manifold and the Euler field $E$ by

$$
\begin{aligned}
& \partial_{1} \circ \underline{\partial}=\underline{\partial} \cdot \mathbf{1}_{3}, \\
& \partial_{2} \circ \underline{\partial}=\underline{\partial} \cdot\left(\begin{array}{ccc}
0 & 0 & 0 \\
1 & 0 & 0 \\
0 & h^{\prime \prime}\left(t_{2}\right) & 0
\end{array}\right), \\
& \partial_{3} \circ \underline{\partial}=\underline{\partial} \cdot\left(\begin{array}{ccc}
0 & 0 & 0 \\
0 & 0 & 0 \\
1 & 0 & 0
\end{array}\right), \\
& E \circ \underline{\partial}=-\underline{\partial} \cdot\left(-t_{1} \cdot \mathbf{1}_{3}+\left(\begin{array}{ccc}
0 & 0 & 0 \\
0 & 0 & 0 \\
t_{3} & 0 & 0
\end{array}\right)\right), \quad \text { so } \quad E=t_{1} \cdot \partial_{1}-t_{3} \cdot \partial_{3} .
\end{aligned}
$$

One can introduce a new coordinate system $\left(\widetilde{t}_{1}, \widetilde{t}_{2}, \widetilde{t}_{3}\right)=\left(t_{1}, \widetilde{t}_{2}, t_{3}\right)$ on the germ $(M, 0)$ with

$$
\partial_{t_{2}}=\frac{1}{\sqrt{h^{\prime \prime}\left(t_{2}\right)}} \cdot \partial_{2}
$$

Denote $\widetilde{\partial}_{j}:=\partial_{\widetilde{t}_{j}}$ and $\underline{\widetilde{\partial}}:=\left(\widetilde{\partial}_{1}, \widetilde{\partial}_{2}, \widetilde{\partial}_{3}\right)=\left(\partial_{1}, \widetilde{\partial}_{2}, \partial_{3}\right)$. Introduce also the new section

$$
\widetilde{v}_{2}:=\frac{1}{\sqrt{h^{\prime \prime}\left(t_{2}\right)}} \cdot v_{2}
$$

and the new basis $\underline{\underline{v}}=\left(\widetilde{v}_{1}, \widetilde{v}_{2}, \widetilde{v}_{3}\right)=\left(v_{1}, \widetilde{v}_{2}, v_{3}\right)$ of the given $(T E)$-structure. Then

$$
\begin{aligned}
& z \nabla_{\widetilde{\partial}_{1}} \underline{\widetilde{v}}=\underline{\widetilde{v}} \cdot \mathbf{1}_{3}, \\
& z \nabla_{\widetilde{\partial}_{2}} \underline{\widetilde{v}}=\underline{\widetilde{v}} \cdot\left(\begin{array}{lll}
0 & 0 & 0 \\
1 & 0 & 0 \\
0 & 1 & 0
\end{array}\right)+\underline{\widetilde{v}} \cdot\left(\begin{array}{ccc}
0 & 0 & 0 \\
0 & \partial_{2} \frac{1}{\sqrt{h^{\prime \prime}\left(t_{2}\right)}} & 0 \\
0 & 0 & 0
\end{array}\right), \\
& z \nabla_{\widetilde{\partial}_{3}} \underline{\widetilde{v}}=\underline{\widetilde{v}} \cdot\left(\begin{array}{ccc}
0 & 0 & 0 \\
0 & 0 & 0 \\
1 & 0 & 0
\end{array}\right), \\
& z^{2} \partial_{z} \underline{\tilde{v}}=\underline{\widetilde{v}} \cdot\left(-t_{1} \cdot \mathbf{1}_{3}+\left(\begin{array}{ccc}
0 & 0 & 0 \\
0 & 0 & 0 \\
t_{3} & 0 & 0
\end{array}\right)\right)+z \cdot \underline{\widetilde{v}} \cdot\left(\begin{array}{ccc}
1 & 0 & 0 \\
0 & 2 & 0 \\
0 & 0 & 3
\end{array}\right) \text {. }
\end{aligned}
$$

In the new coordinates the multiplication becomes simpler and independent of the choice of $h\left(t_{2}\right)$ (as long as $h^{\prime \prime}\left(t_{2}\right) \neq 0$ ):

$$
\begin{aligned}
& \widetilde{\partial}_{1} \circ \underline{\widetilde{\partial}}=\underline{\widetilde{\partial}} \cdot \mathbf{1}_{3}, \\
& \widetilde{\partial}_{2} \circ \underline{\widetilde{\partial}}=\underline{\widetilde{\partial}} \cdot\left(\begin{array}{lll}
0 & 0 & 0 \\
1 & 0 & 0 \\
0 & 1 & 0
\end{array}\right),
\end{aligned}
$$




$$
\begin{aligned}
& \widetilde{\partial}_{3} \circ \underline{\widetilde{\partial}}=\underline{\widetilde{\partial}} \cdot\left(\begin{array}{ccc}
0 & 0 & 0 \\
0 & 0 & 0 \\
1 & 0 & 0
\end{array}\right), \\
& E \circ \underline{\widetilde{\partial}}=-\underline{\widetilde{\partial}} \cdot\left(-t_{1} \cdot \mathbf{1}_{3}+\left(\begin{array}{ccc}
0 & 0 & 0 \\
0 & 0 & 0 \\
t_{3} & 0 & 0
\end{array}\right)\right), \\
& \text { so } E=t_{1} \cdot \widetilde{\partial}_{1}-t_{3} \cdot \widetilde{\partial}_{3} .
\end{aligned}
$$

This is the nilpotent $F$-manifold for $n=3$ in [4, Theorem 3]. However, the Euler field here is different from the one in [4, Theorem 3]. The endomorphism Eo here is not regular, but has only the one eigenvalue $t_{1}$ and has for $t_{3} \neq 0$ one Jordan block of size $2 \times 2$ and one Jordan block of size $1 \times 1$ and is semisimple for $t_{3}=0$.

The sections $v_{1}, v_{2}, v_{3}$ define also an extension $\widehat{H} \rightarrow \mathbb{P}^{1}$ such that the $(T E)$-structure extends to a pure $(T L E)$-structure.

Furthermore $v$ satisfies all properties of the section $\zeta$ in Theorem 6.6(b) in [7]. Thus the $F$-manifold with Euler field is enriched to a flat $F$-manifold with Euler field (Definition 3.1(b) in [7]).

If we try to introduce a pairing which would make it into a pure (TLEP)-structure, we get a constraint $h^{\prime \prime}\left(t_{2}\right)=$ const. However, probably similar higher dimensional examples allow also an extension to pure (TLEP)-structures while keeping the functional freedom. This would give families of Frobenius manifolds with Euler fields with functional freedom on a fixed $F$-manifold with Euler field.

In the example above, $t_{1}, t_{2}, t_{3}$ are flat coordinates and $\widetilde{t}_{1}=t_{1}, \widetilde{t}_{2}, \widetilde{t}_{3}=t_{3}$ are generalized canonical coordinates (in which the multiplication has simple formulas).

\section{Acknowledgements}

This work was funded by the Deutsche Forschungsgemeinschaft (DFG, German Research Foundation) - 242588615. I would like to thank Liana David for a lot of joint work on (TE)-structures.

\section{References}

[1] Anosov D.V., Bolibruch A.A., The Riemann-Hilbert problem, Aspects of Mathematics, Vol. 22, Friedr. Vieweg \& Sohn, Braunschweig, 1994.

[2] Babbitt D.G., Varadarajan V.S., Formal reduction theory of meromorphic differential equations: a group theoretic view, Pacific J. Math. 109 (1983), 1-80.

[3] Cotti G., Dubrovin B., Guzzetti D., Isomonodromy deformations at an irregular singularity with coalescing eigenvalues, Duke Math. J. 168 (2019), 967-1108, arXiv:1706.04808.

[4] David L., Hertling C., Regular F-manifolds: initial conditions and Frobenius metrics, Ann. Sc. Norm. Super. Pisa Cl. Sci. (5) $\mathbf{1 7}$ (2017), 1121-1152, arXiv:1411.4553.

[5] David L., Hertling C., (T)-structures over two-dimensional F-manifolds: formal classification, Ann. Mat. Pura Appl. (4) 199 (2020), 1221-1242, arXiv:1811.03406.

[6] David L., Hertling C., (TE)-structures over the irreducible 2-dimensional globally nilpotent $F$-manifold germ, Rev. Roumaine Math. Pures Appl. 65 (2020), 235-284, arXiv:2001.01063.

[7] David L., Hertling C., Meromorphic connections over F-manifolds, in Integrability, Quantization, and Geometry. I. Integrable systems, Proceedings of Symposia in Pure Mathematics, Vol. 103, Editors S. Novikov, I. Krichever, O. Ogievetsky, S. Shlosman, Amer. Math. Soc., Providence, RI, 2021, 171-216, arXiv:1912.03331.

[8] Fabry E., Sur les intégrales des équations différentielles lineaires à coefficients rationels, Ph.D. Thesis, Paris, 1885. 
[9] Hertling C., Frobenius manifolds and moduli spaces for singularities, Cambridge Tracts in Mathematics, Vol. 151, Cambridge University Press, Cambridge, 2002.

[10] Hertling C., $t t^{*}$ geometry, Frobenius manifolds, their connections, and the construction for singularities, J. Reine Angew. Math. 555 (2003), 77-161, arXiv:math.AG/0203054.

[11] Hertling C., Hoevenaars L., Posthuma H., Frobenius manifolds, projective special geometry and Hitchin systems, J. Reine Angew. Math. 649 (2010), 117-165, arXiv:0905.3304.

[12] Hertling C., Manin Yu., Weak Frobenius manifolds, Int. Math. Res. Not 1999 (1999), 277-286, arXiv:math.QA/9810132.

[13] Hertling C., Manin Yu., Unfoldings of meromorphic connections and a construction of Frobenius manifolds, in Frobenius Manifolds, Aspects Math., Vol. 36, Friedr. Vieweg, Wiesbaden, 2004, 113-144, arXiv:math.AG/0207089.

[14] Hertling C., Sabbah C., Examples of non-commutative Hodge structures, J. Inst. Math. Jussieu 10 (2011), 635-674, arXiv:0912.2754.

[15] Hertling C., Sevenheck C., Limits of families of Brieskorn lattices and compactified classifying spaces, Adv. Math. 223 (2010), 1155-1224, arXiv:0805.4777.

[16] Malgrange B., La classification des connexions irrégulières à une variable, in Mathematics and Physics (Paris, 1979/1982), Progr. Math., Vol. 37, Birkhäuser Boston, Boston, MA, 1983, 381-399.

[17] Malgrange B., Sur les déformations isomonodromiques. I. II. Singularités irrégulières, in Mathematics and physics (Paris, 1979/1982), Progr. Math., Vol. 37, Birkhäuser Boston, Boston, MA, 1983, 401-438.

[18] Malgrange B., Deformations of differential systems. II, J. Ramanujan Math. Soc. 1 (1986), 3-15.

[19] Sabbah C., Introduction to algebraic theory of linear systems of differential equations, in Éléments de la théorie des systèmes différentiels. D-modules cohérents et holonomes (Nice, 1990), Travaux en Cours, Vol. 45, Hermann, Paris, 1993, 1-80.

[20] Sabbah C., Polarizable twistor D-modules, Astérisque 300 (2005), vi+208 pages, arXiv:math.AG/0503038.

[21] Sabbah C., Isomonodromic deformations and Frobenius manifolds. An introduction, Universitext, Springer, London, 2008.

[22] Sabbah C., Integrable deformations and degenerations of some irregular singularities, Publ. Res. Inst. Math. Sci., to appear, arXiv:1711.08514.

[23] Saito M., Deformations of abstract Brieskorn lattices, arXiv:1707.07480.

[24] Varadarajan V.S., Linear meromorphic differential equations: a modern point of view, Bull. Amer. Math. Soc. (N.S.) 33 (1996), 1-42. 